\title{
APPLICATION OF CHEBYSHEV FORMALISM TO IDENTIFY NONLINEAR MAGNETIC FIELD COMPONENTS IN BEAM TRANSPORT SYSTEMS
}

\author{
by \\ Michael Spata \\ B.S. June 1990, SUNY, Stony Brook \\ M.S. May 2008, Old Dominion University
}

A Dissertation Submitted to the Faculty of Old Dominion University in Partial Fulfillment of the Requirements for the Degree of

DOCTOR OF PHILOSOPHY

PHYSICS

OLD DOMINION UNIVERSITY

August 2012

Approved by:

Geoffrey Krafft (Director)

Hari Areti (Member)

Ravindra Joshi (Member)

Reza Kazimi (Member)

Sebastian Kuhn (Member) 


\title{
ABSTRACT \\ APPLICATION OF CHEBYSHEV FORMALISM TO IDENTIFY NONLINEAR MAGNETIC FIELD COMPONENTS IN BEAM TRANSPORT SYSTEMS
}

\author{
Michael Spata \\ Old Dominion University, 2012 \\ Director: Dr. Geoffrey Krafft
}

An experiment was conducted at Jefferson Lab's Continuous Electron Beam Accelerator Facility to develop a beam-based technique for characterizing the extent of the nonlinearity of the magnetic fields of a beam transport system. Horizontally and vertically oriented pairs of air-core kicker magnets were simultaneously driven at two different frequencies to provide a time-dependent transverse modulation of the beam orbit relative to the unperturbed reference orbit. Fourier decomposition of the position data at eight different points along the beamline was then used to measure the amplitude of these frequencies. For a purely linear transport system one expects to find solely the frequencies that were applied to the kickers with amplitudes that depend on the phase advance of the lattice. In the presence of nonlinear fields one expects to also find harmonics of the driving frequencies that depend on the order of the nonlinearity. Chebyshev polynomials and their unique properties allow one to directly quantify the magnitude of the nonlinearity with the minimum error. A calibration standard was developed using one of the sextupole magnets in a CEBAF beamline. The technique was then applied to a pair of Arc 1 dipoles and then to the magnets in the Transport Recombiner beamline to measure their multipole content as a function of transverse position within the magnets. 
Copyright, 2012, by Michael Spata, All Rights Reserved. 


\section{ACKNOWLEDGEMENTS}

I have been very fortunate to work at a scientific facility such as Jefferson Lab where the opportunity to pursue an advanced degree while continuing to work is made possible. I would like to thank the Jefferson Lab management for making this significant investment in me. I also must thank Dr. Andrew Hutton, Dr. Swapan Chattophadyay, Dr. Hari Areti, and Dr. Larry Weinstein for their encouragement to pursue a Ph.D. at Old Dominion University.

I would like to thank my thesis advisor and Jefferson Lab supervisor Dr. Geoffrey Krafft for creating an environment where I could complete my studies while working full time at the laboratory. The experimental technique used for this research originated with Dr. Krafft's beam-based experiments of the CEBAF Injector during the commissioning of the $4 \mathrm{GeV}$ machine. It has been very rewarding to have had the opportunity to extend these techniques and to learn under his direction. I am indebted to my committee for their acceptance of my original proposal, their guidance during the course of my work and their critical review of the final manuscript. I thank Dr. Hari Areti, Dr. Ravindra Joshi, Dr. Reza Kazimi, and Dr. Sebastian Kuhn for serving in this regard.

The data acquisition hardware used for this experiment was a prototype for a system being developed by Trent Allison, one of the Electrical Engineers at Jefferson Lab. Trent made extensive modifications to the system firmware and hardware to meet the needs of this experiment. He also worked tirelessly to teach me how it works and how to use it. I believe Trent was as excited as I was the first time we got data out of the system.

I would like to thank Jeff Dail from the Machine Shop for fabricating the MAKstyle kicker magnets for the experiment. Jeff machined the coil winding fixture, wound and potted the 150-turn coils and machined the clamps for installing them on the beamline. Jeff is an excellent machinist and it was a pleasure to collaborate with him during the design phase. The final product met all of the specifications and was delivered on time despite there being many other higher priority jobs in the queue.

I am very grateful for the support that I received from the Accelerator Operations Group throughout the experiment. Thanks to Dr. Arne Freyberger, the Director of Operations, for providing the funding for kicker magnet fabrication and for allocating sufficient beam time to conduct this research. I would like to thank Operations Crew 
Chief Darrell Spraggins who invariably seemed to be supervising the Control Room Operators on the days that I was to have beam. During most of the time that I was performing beam tests I was in the field and away from the Control Room. I am thankful to have had the excellent support of the Accelerator Operators during the tests. Thanks to Brian Connolly, Eric Forman and Shawn Frierson for their support during this experiment.

I was very fortunate to have had the support of the DC Power and Controls Software Groups for getting the magnets up and running. Thanks to Associate Coordinator Simon Wood and Technicians Lee Broeker, Mark Todd and Maximo Tan for their help in getting the control system hardware installed for the sextupole and kicker magnets. Thanks to Electrical Engineer Sarin Philip for his analysis and field support for getting the MAK magnets matched to the magnet power supplies. And many thanks to Computer Scientist Scott Higgins for providing the EPICS software support to control the kicker and sextupole magnets.

I would like to thank Engineers Ken Baggett and Joe Myers as well as Technician Steve Ellis from our Magnet Measurement Facility for all of their support. Ken and his team provided the magnetic field measurements and temperature data for the MAK kickers. Ken also provided the historic data for the Arc 1 dipoles.

I am indebted to my Accelerator Physicist colleagues for many fruitful discussions and for providing analysis support. Dr. Yves Roblin provided excellent training on the Elegant modeling software and helped me get the simulations of my experiment running. Dr. Jay Benesch provided TOSCA analysis of an Arc 1 Dipole and helped me understand multipole expansions. Dr. Balsa Terzic introduced me to the NAFF algorithm and also provided some software tools for doing the analysis.

I would especially like to thank Dr. Alicia Hofler for helping me get started with Poisson simulations. Being a recent graduate, as well as a friend and colleague for many years, I was able to benefit from her great sense of humor and her experience in pursuing a $\mathrm{Ph} . \mathrm{D}$.

I would like to thank Associate Coordinator Pete Francis from the Electrical Engineering Support Group. Pete is responsible for the Beam Position Monitor system and helped me get my data acquisition hardware connected in the field. Pete also helped me move all of the data acquisition gear from one side of the site to the other. 
I am grateful for having had the opportunity to work with Engineers John Musson, Trent Allison and Jianxun Yan as well as Technician Keith Cole on the Beam Position Monitor Stretched-Wire Test Stand. This system was used to measure the nonlinearity of the M15 and M20 Beam Position Monitors which was an essential part of this research.

Last of all, and closest to me heart, I must thank my family. I owe my deepest gratitude to my wonderful wife Sarah and my amazing daughters Samantha and Emma. Balancing family life with work and school is always a challenge. My family's love and patience has helped me in so many way during the course of my graduate studies. Also, many thanks to my Mom and Dad. This achievement would not have been possible without the love and support they have provided through the years. 


\section{TABLE OF CONTENTS}

Page

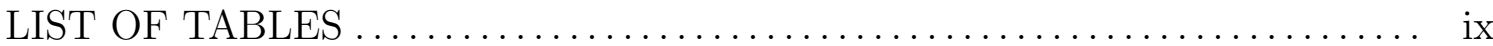

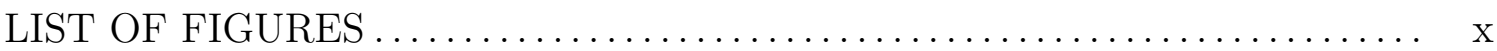

Chapter

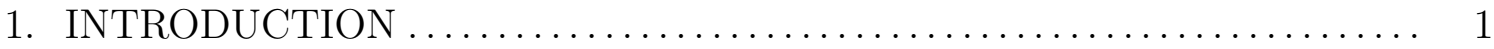

2. THE CEBAF ACCELERATOR ............................. 4

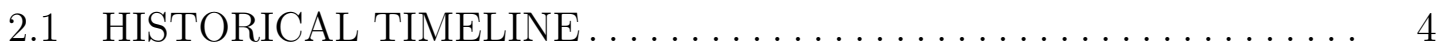

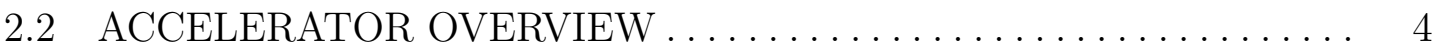

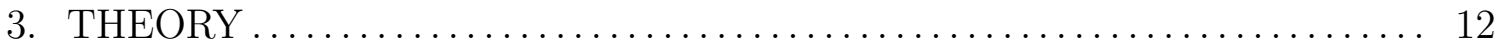

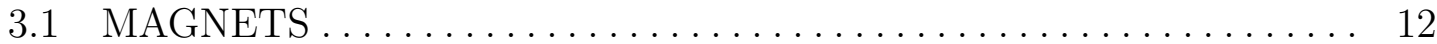

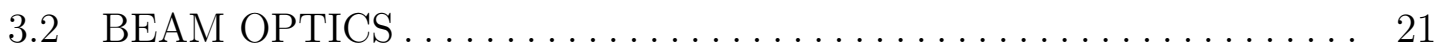

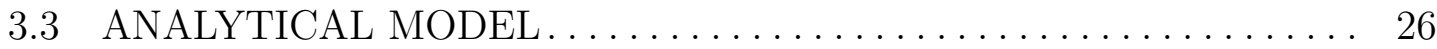

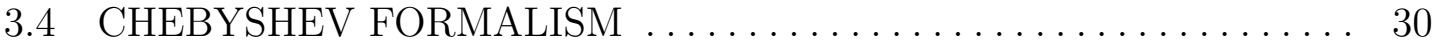

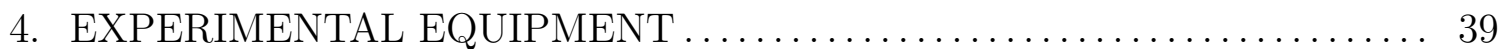

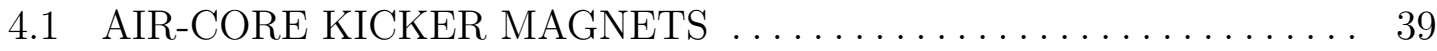

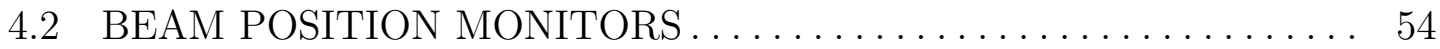

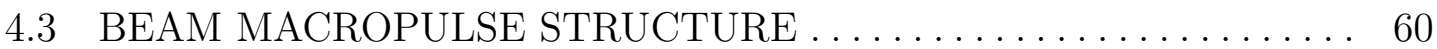

4.4 DATA ACQUISITION SYSTEM HARDWARE . . . . . . . . . . . . 61

5. LABORATORY MEASUREMENTS AND SIMULATIONS . ......... 68

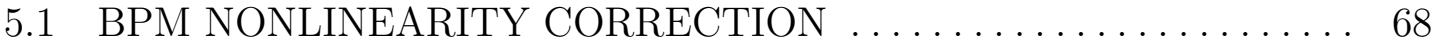

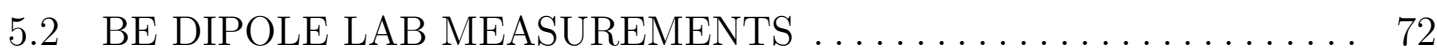

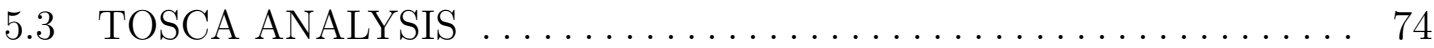

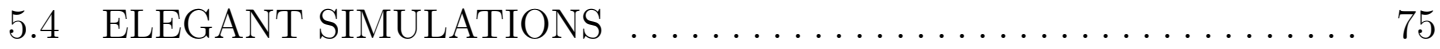

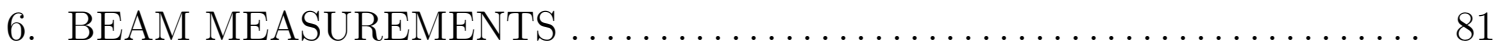

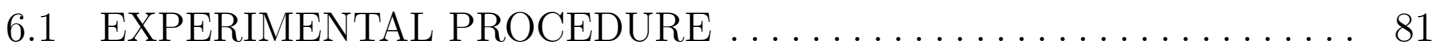

6.2 NAFF ALGORITHM . . . . . . . . . . . . . . . . . . . . . 84

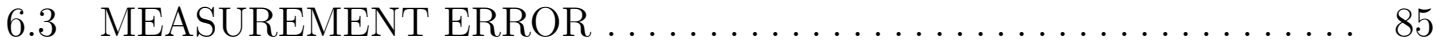

6.4 SEXTUPOLE MEASUREMENTS . . . . . . . . . . . . . . . . 91

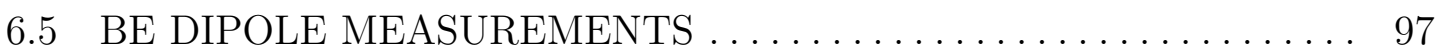

6.6 BSY RECOMBINER MEASUREMENTS $\ldots \ldots \ldots \ldots \ldots \ldots \ldots \ldots . . \ldots 7$

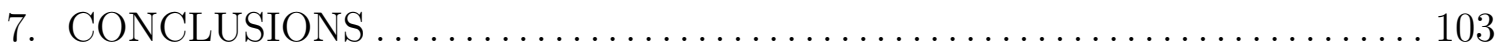

7.1 SUMMARY OF EXPERIMENT . . . . . . . . . . . . . . . . 103

7.2 SUGGESTIONS FOR FUTURE WORK . . . . . . . . . . . . 105 


\section{APPENDICES}

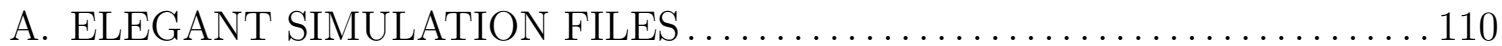

A.1 ARC 1 LATTICE FILE $\ldots \ldots \ldots \ldots \ldots \ldots \ldots \ldots \ldots \ldots \ldots \ldots \ldots \ldots$

A.2 ARC1 ELEGANT FILE ................................ 116

B. MATLAB CODE FOR ANALYZING BPM WIRE DATA $\ldots \ldots \ldots \ldots \ldots \ldots 118$

C. POISSON CODE FOR SIMUlating BPM $\ldots \ldots \ldots \ldots \ldots \ldots \ldots \ldots \ldots \ldots$

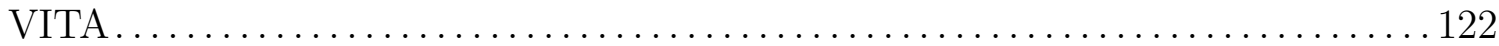




\section{LIST OF TABLES}

Table

Page

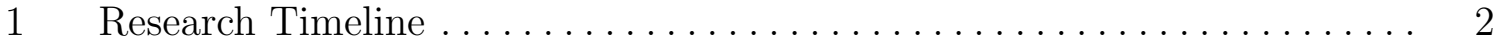

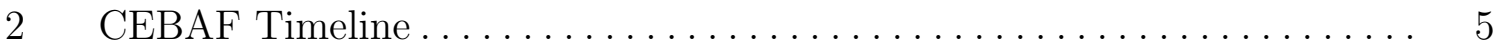

3 Harmonic sidebands for fundamental frequencies of $1 \mathrm{~Hz}$ and $21 \mathrm{~Hz} . \ldots 30$

4 Chebyshev Polynomials from $T_{0}(x)$ through $T_{10}(x) \ldots \ldots \ldots \ldots \ldots \ldots$

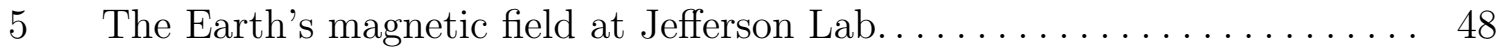

$6 \quad$ BPM Wire Data with Calibration Oscillators On . . . . . . . . . . 58

$7 \quad$ BPM Wire Data with Calibration Oscillators Off . . . . . . . . . . . . 59

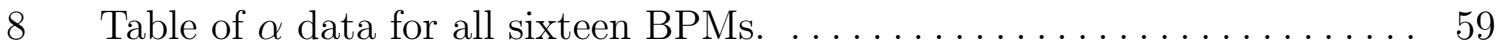

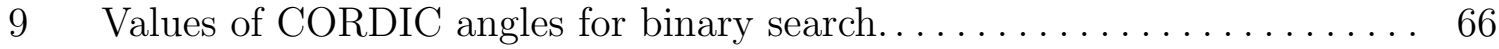

10 Spectral data for $y-y^{\prime}$ modulation in Sextupole mid-plane . . . . . . . . 94

11 Spectral data for $x-x^{\prime}$ modulation in Sextupole mid-plane . . . . . . . . . 94

12 Spectral data for $y-y^{\prime}$ modulation vs. $x$ position in BE Dipole . . . . . 98 


\section{LIST OF FIGURES}

Figure

1 A schematic layout of the CEBAF accelerator showing the characteristic racetrack design (Drawing courtesy of Tom Oren) . . . . . . . . . . .

2 A photograph of one of the CEBAF linear accelerators. Each of the linac's twenty cryomodules operate at $2 \mathrm{~K}$ and provide an average energy gain of $30 \mathrm{MeV}$ (Photo from JLAB archive) . . . . . . . . . . . . . .

3 A photograph of one of the CEBAF 5-cell niobium cavities. The elliptical cells have an active length of $0.5 \mathrm{~m}$ with each cavity providing an average energy gain of $3.75 \mathrm{MeV}$ (Photo from JLAB archive). . . . . . . . .

4 A photograph of a section of the East Arc. The lowest energy Arc 1 beamline is at the top with Arcs 3,5,7 and 9 stacked below (Photo from

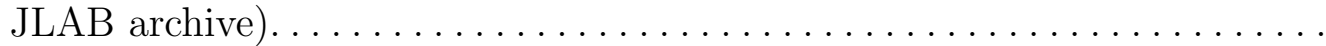

5 A photograph showing stacks of magnet assemblies in the East Arc. The order of elements is sextupole, BPM, quadrupole and corrector (Photo

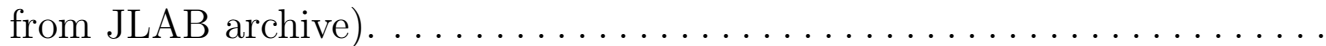

6 Schematic diagram of an ideal dipole (left) and an example of a C-shaped dipole showing the path of integration used to determine the magnetic

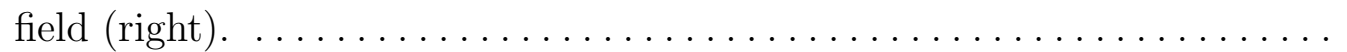

7 A detailed drawing of the beam aperture of a quadrupole showing the direction of current flow, the potential for each pole, the field lines within the aperture and the direction of force for an electron. . . . . . . . . . . .

8 A detailed drawing of the beam aperture of a sextupole showing the direction of current flow, the potential for each pole, the field lines within the aperture and the direction of force for an electron. . . . . . . . . . .

9 A detailed drawing of the beam aperture of an octupole showing the direction of current flow, the potential for each pole, the field lines within the aperture and the direction of force for an electron. . . . . . . . . . . .

10 Schematic diagram of a quadrupole (left) and a detailed picture of one of the poles showing the path of integration used to determine the magnetic

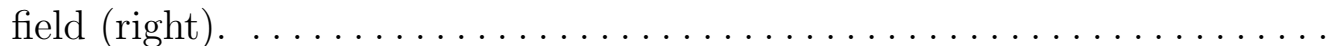


11 Schematic diagram of a sextupole (left) and a detailed picture of one of the poles showing the path of integration used to determine the magnetic

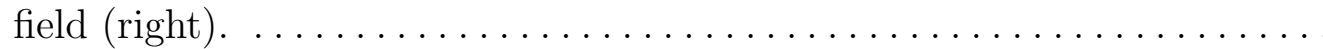

12 An arbitrary sequence of beamline elements. . . . . . . . . . . . .

13 A simple model of beamline elements for demonstrating how two distinct frequencies of a simultaneously modulated electron beam will mix due to

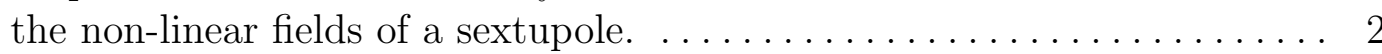

14 A plot of Chebyshev polynomials $T_{1}(x)$ through $T_{5}(x) \ldots \ldots \ldots \ldots \ldots$

15 The plot at the left shows an arbitrary function to fit three points using a cosine expansion. The plot at the right shows harmonic lines from the

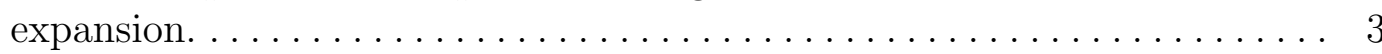

16 A schematic representation of the experiment...............

17 A picture showing two of the 8" long 60-turn kicker magnets in Arc 1 at the top and two of the 14" long 150-turn kicker magnets in the Arc 6

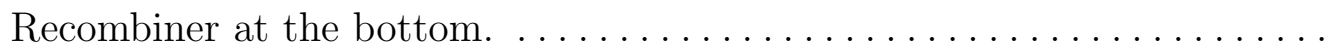

18 The coordinate system for determining the magnetic field a distance $R$ from a long straight wire carrying a current $I \ldots \ldots \ldots \ldots \ldots \ldots \ldots \ldots . \ldots \ldots$

19 A cross-sectional view of an MAK magnet showing the field vectors for each of the four current sources and the resultant vertical field. . . . . . . 43

20 A picture of the winding fixture that was used to make the coils. . . . . . . 44

21 A drawing of the coil design for the 150 turn MAK kicker magnets that were used in Arc 6. An elevation view (top) and plan view (bottom) is

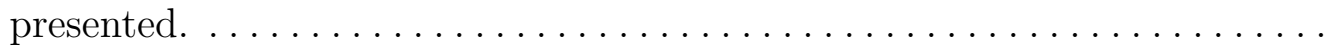

22 A drawing of the aluminium clamp for mounting the correctors on the 3" beam tube. At left is a downstream view and shows the coils in the mount. At right is an edge view to show the channel for the stainless steel

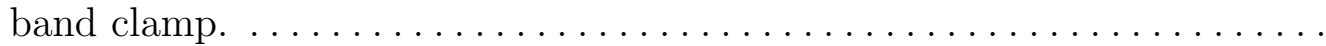

23 A graph of temperature data for a coil pair operating at 5 Amps DC current. 46

24 Field map graphs for the MAZ magnet at the top and the MAK magnet at the bottom. Both data are integrated along $z$ at $x=y=0 \ldots \ldots \ldots .47$

25 The generalized coordinate system for describing the earth's field. . . . . . . 
26 A graph of the magnetic field versus the longitudinal position for the MAK magnet showing all thirteen tracks. The box represents the length

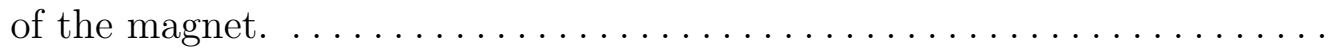

27 A graph of the integrated field strength versus the transverse position in the midplane. The data is normalized to the track at $x=y=0 \ldots \ldots$.

28 Harmonic content of MAZ kicker for $1.0 \mathrm{~cm}$ radius at the top and $0.5 \mathrm{~cm}$

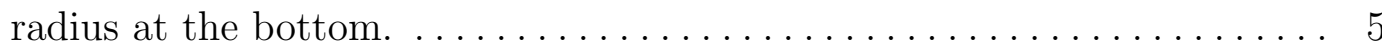

29 Harmonic content of MAK kicker for $1.0 \mathrm{~cm}$ radius at the top and $0.5 \mathrm{~cm}$

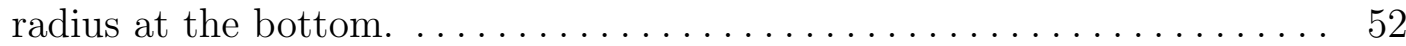

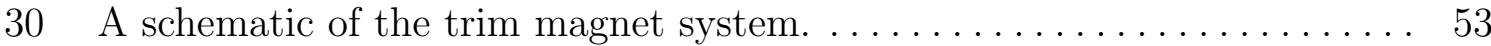

31 Schematic drawing of an M20 BPM at the top and an M15 BPM at the

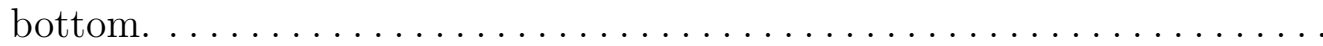

32 An electrical schematic for a CEBAF Beam Position Monitor. The electron beam creates a transverse electromagnetic field that couples to the $V^{+}$and $V^{-}$antennae. The measured position will depend on the differ-

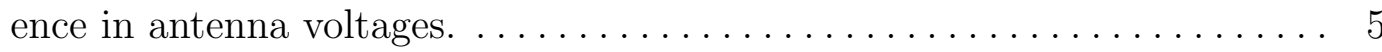

33 A plot of four BPM wire signals during the alpha calibration procedure. . 57

34 A schematic layout of the BPM Electronics. . . . . . . . . . . . . . 58

35 The beam pulse structure used for tuning the machine is shown at the top. The beam pulse structure during modulation experiments is shown

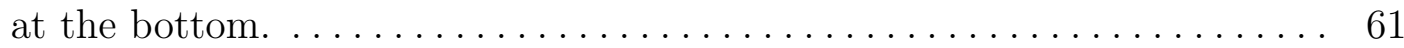

36 A picture of the data acquisition system in the service building. . . . . . . .

37 A picture of one of the four data acquisition circuit boards. . . . . . . . 63

38 A schematic representation of the data flow through an ADC board..... 63

$39 \quad I-Q$ sampling as a function of time shown at the right. A phasor diagram showing the same sampling is shown at the left. ........... 64

40 A drawing showing the $I-Q$ sampling scheme used by the data acquisition

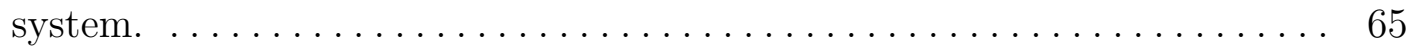

41 Illustration of binary search pattern for finding magnitude and phase. . . 66

42 A picture of the BPM test stand with an M15 BPM mounted on the stage

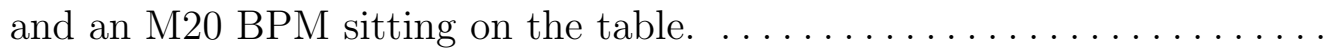


43 Data from the BPM test stand showing the nonlinear response and the nonlinear potential map that was generated using Poisson. . . . . . . . 70

44 A sequence of plots showing the distortion of BPM data due to the limitations of the difference/sum method and the results of the correction....

45 Beam modulation in $y$-plane at left and a histogram of position corrections

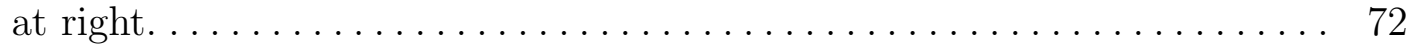

46 A sketch of the grid of points and the coordinate system for calculating

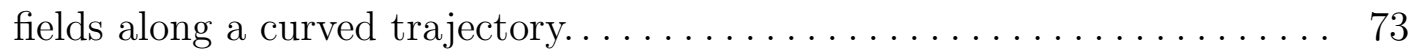

47 A plot of relative sextupole strength vs. transverse position for a BE dipole. 74

48 The 3-D Tosca model of a CEBAF BE-style dipole. The iron above the

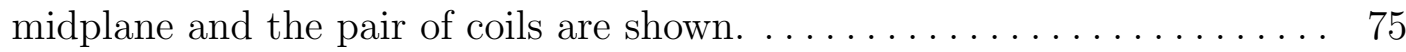

49 The arrangement of the eighteen orbits through the BE dipole and the eighteen points around the circle used for TOSCA analysis. . . . . . . . 76

50 The integrated relative sextupole strength is plotted vs. the index of the

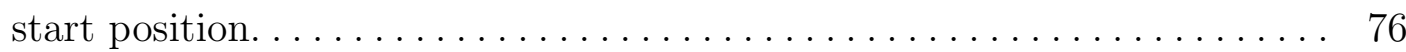

51 Horizontal position and the FFT downstream of a sextupole at $1000 \mathrm{G} / \mathrm{cm} .79$

52 Simulation results for the sextupole study and dipole study. . . . . . . . . 80

53 Typical beam centroid stability showing the $x$ position as a function of time for Beam Position Monitor IPM1A16... . . . . . . . . . . . . . 83

54 A plot of the relative error in amplitude for FFT versus NAFF for the 20 and $22 \mathrm{~Hz}$ sidebands as a function of sextupole strength. . . . . . . . 85

55 A plot of the amplitude of the $20 \mathrm{~Hz}$ peak for $y-y^{\prime}$ modulation as a

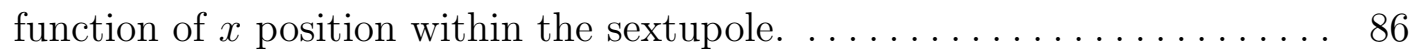

56 Plot of $x$ and $y$ centroid noise at IPM1A16. The main source of peak broadening is due to $\mathrm{AC}$ ripple on magnet currents. . . . . . . . . . 87

57 FFT of $x$ and $y$ centroid noise at IPM1A16 showing the amplitude of the

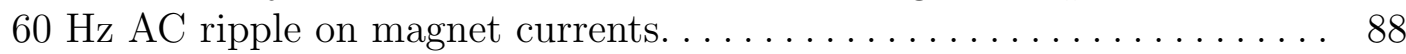

58 The few parts in a thousand amplitude of the residual peaks in the absence of sextupole excitation sets a lower bound on the measurement error. . . . . 
59 Upper plot shows the spectra with the sextupole set to $1000 \mathrm{G} / \mathrm{cm}$. Lower plot with sextupole ramped to zero showing only the remnant field in the

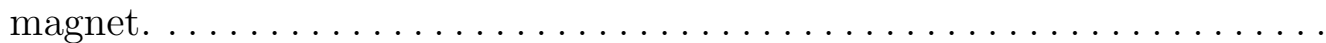

60 Plots of the transverse position for all eight BPMs during a sextupole run with $y-y^{\prime}$ modulation. Cross plane coupling is evident starting at 1A16. 92

61 Plots of the transverse position for all eight BPMs during a sextupole run with $x-x^{\prime}$ modulation. No cross plane coupling occurs. . . . . . . . . . . 93

62 Beam data for the sextupole measurements with $y$-plane modulation. The $2 \mathrm{~Hz}$ and $42 \mathrm{~Hz}$ data are at the top and the $20 \mathrm{~Hz}$ and $22 \mathrm{~Hz}$ data are at

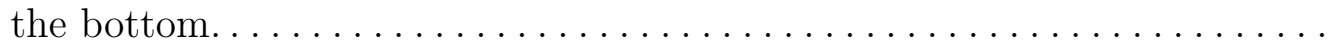

63 Beam data for the sextupole measurements with $x$-plane modulation. The $2 \mathrm{~Hz}$ and $42 \mathrm{~Hz}$ data are at the top and the $20 \mathrm{~Hz}$ and $22 \mathrm{~Hz}$ data are at

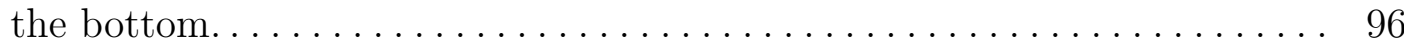

64 Beam data for $y$-plane modulation in a pair of BE dipoles. . . . . . . . . 98

65 Plots of small, medium and large beam modulations as measured at the

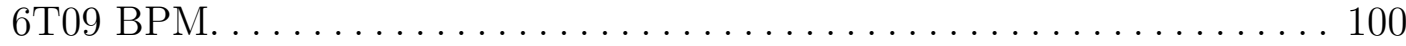

66 Series of FFT plots of the 6T09 BPM for $x-x^{\prime}$ modulation. . . . . . . . 101

67 Series of FFT plots of the 6T09 BPM for $y-y^{\prime}$ modulation. . . . . . . . 102 


\section{CHAPTER 1}

\section{INTRODUCTION}

In this thesis a technique for characterizing the extent of the nonlinearity of the magnets of beam transport systems is investigated both theoretically and experimentally. There are well over 2200 magnets in the CEBAF accelerator with more than 50 distinct types. The fields of these dipole and quadrupole magnets are specified and designed to be linear across the aperture that the electron beam occupies. Errors in the real magnetic field of these beamline elements relative to an ideal model can occur for several reasons.

Symmetry conditions allow the existence of certain systematic errors and forbid others depending on the magnet type. For example in addition to the quadrupole edge focussing of a dipole there is also an allowed second order sextupole term. In addition to these systematic multipole errors one can also find random errors that can be attributed to deficiencies in assembly, manufacturing or powering.

Another source of error in the machine is misalignment of these components relative to the ideal model. The accelerator design specifies the transverse and longitudinal location of all of these beamline elements with a precision of 10 microns. The real machine can only be aligned to within 250 microns of the design. These positional errors can be compounded by roll, tilt or yaw errors in the angle of the dipoles and quadrupoles. Errors of this sort result in cross-plane coupling of the beam transport which can be very difficult to manage in the real machine.

Traditional methods of tuning the accelerator to account for errors in the linear optics have utilized discrete transverse perturbations of the beam's trajectory relative to the design trajectory. Starting from the initial point in the lattice where the transverse kick occurs, the beam position will oscillate about the reference trajectory with an amplitude and phase that depend on the quasi-periodic focussing strength of the lattice. The phase and amplitude of these oscillations are compared to a design model while tuning quadrupoles at key locations are adjusted to minimize errors.

To detect the nonlinear errors of the lattice one must use nonlinear perturbation

techniques. Simultaneous sinusoidal modulation of the beam at two locations using 
TABLE 1. Research Timeline

\begin{tabular}{cc}
\hline \hline Time Period & Milestone \\
\hline July 2009 & Thesis Proposal Presented to the Committee \\
Fall 2009 & Data Acquisition System Development and Installation in Arc 1 \\
December 2009 & First Test Run in Arc 1 to Characterize MAZ Kicker Magnets \\
March 2010 & Sextupole Calibration Beam Test in Arc 1 \\
April 2010 & Thesis Update Presented to the Committee \\
July 2010 & Sextupole and Dipole Measurements in Arc 1 \\
Fall 2010 & Design, Fabricate and Measure MAK Kicker Magnets \\
December 2010 & Install Kicker Magnets in Arc 6 Recombiner \\
January 2011 & Move Data Acquisition System from Arc 1 to Arc 6 \\
February 2011 & First Test Run in Arc 6 Recombiner to Commission Kicker Magnets \\
April 2011 & First Beam Test in Arc 6 Recombiner \\
May 2011 & Final Set of Arc 6 Recombiner Measurements \\
Summer 2011 & BPM Test Stand Development and Linearity Studies \\
September 2011 & Presentation at the International Particle Accelerator Conference \\
April 2012 & Thesis Defense \\
\hline \hline
\end{tabular}

two distinct frequencies is used in this thesis to identify the nonlinear fields in the lattice.

In Table $1 \mathrm{a}$ timeline for this research is presented to provide an account of what was accomplished over the last three years. The rest of this introduction gives an overview of the chapters that follow.

In the second chapter an introduction to the CEBAF accelerator is provided. The first section provides an historical overview of the facility and a look ahead towards the $12 \mathrm{GeV}$ Upgrade. This is followed by a more technical description of the accelerator.

The third chapter provides the theoretical basis for the experiment. First comes the derivation of the functional form of the magnetic fields for dipoles, quadrupoles and sextupoles as well as the two-dimensional general multipole expansion. This is followed by a description of beam optics and the matrix formalism that is central to research in accelerator physics. The development of a simple beamline model to demonstrate how simultaneous sinusoidal beam modulations will mix in the presence of non-linear magnetic fields is then provided. The chapter finishes with a discussion of Chebyshev polynomials and their unique properties for minimizing errors in 
modulation experiments.

The fourth chapter describes the experimental equipment that was used for this research. The design, fabrication and measurement of the AC kicker magnets that were used to modulate the beam is presented. This is followed by a description of the Beam Position Monitors that are used to measure the position and modulation pattern of the electron beam. Next comes a description of the beam timing structure that was used for this experiment. The last section gives an overall description of the data acquisition system.

The fifth chapter describes the experimental measurements and simulations that were done in support of this research. First comes a discussion on the analysis and correction of the nonlinearity of the Beam Position Monitors. This is followed by a description of the experimental procedures used for taking data. The next section presents the magnetic field measurements, from the Jefferson Lab's Magnet Measurement Facility, of an Arc 1 dipole. The chapter finishes with a discussion of simulations that were conducted as part of this research.

The sixth chapter provides a description of the analysis and presents the results for the sextupole calibration runs, the Arc 1 dipole measurements as well as the results from the study of the Arc 6 Recombiner beamline.

The seventh chapter provides some conclusions and a summary for the work presented in this thesis. 


\section{CHAPTER 2}

\section{THE CEBAF ACCELERATOR}

\subsection{HISTORICAL TIMELINE}

CEBAF was designed and constructed for the Department of Energy (DOE) as a $4 \mathrm{GeV}, 200 \mu \mathrm{A}$ five pass, recirculating electron accelerator and has been operating for Nuclear Physics research since November 1995. A timeline showing some important milestones for the facility is shown in Table 2. The initial construction took a little over six years with the machine being fully installed by 1993. An intense two year commissioning period culminated with the first five pass CW beam delivered to experimental Hall C on 25 May 1995.

CEBAF reached its design goal of $1 \mathrm{MW}$ of beam power a year later. Beamlines to experimental Halls $\mathrm{A}$ and $\mathrm{B}$ were completed and commissioned over the next two years with first beam delivery in 1997. Simultaneous three-hall operations was achieved in 1998 .

The energy reach of the accelerator was enhanced to $6 \mathrm{GeV}$ beginning in 2000 through a multi-year refurbishment program of $25 \%$ of the machine's two linear accelerators. Since then the facility has been conducting a robust Nuclear Physics program for over thirty weeks a year at energies up to $6 \mathrm{GeV}$.

While the laboratory was first starting up the $6 \mathrm{GeV}$ program proposals were already being developed to double the machine's energy to $12 \mathrm{GeV}$ and to add a fourth experimental Hall D. The DOE accepted the proposal and provided first funding for the conceptual design phase in 2004. Approval to begin the engineering and design phase of the project came in 2006. Construction for the $\$ 310$ million dollar project began in September of 2008. During an extended shutdown in 2011 the laboratory completed the first phase of the $12 \mathrm{GeV}$ upgrade and then resumed the $6 \mathrm{GeV}$ program for a final run which ended on 18 May 2012. The accelerator then shut down for an eighteen month shutdown to finish the $12 \mathrm{GeV}$ Upgrade. Accelerator commissioning is scheduled to begin in October of 2013 with all beamlines completed by 2016 . 
TABLE 2. CEBAF Timeline

\begin{tabular}{lc}
\hline \hline Year & Milestone \\
\hline 1984 & Site selection and first DOE funding provided for CEBAF \\
1987 & Construction begins on the new facility \\
1991 & Injector beamline is installed and commissioned \\
1993 & Linear accelerators and all 6 km of beamline are installed \\
1995 & First 4 GeV CW beam delivery to experimental Hall C \\
1996 & CEBAF reaches 1 MW of beam power \\
1997 & First beam delivery to experimental Hall A \\
1997 & First beam delivery to experimental Hall B \\
1998 & Simultaneous three-hall operations at $4 \mathrm{GeV}$ \\
2000 & CEBAF reaches 6 GeV with 10 refurbished cryomodules \\
2004 & 12 GeV Upgrade is funded and engineering/design work begins \\
2011 & First phase of $12 \mathrm{GeV} \mathrm{Upgrade} \mathrm{completed}$ \\
2012 & Final 6 GeV run with beam to Halls A,B,C ended in May \\
2013 & Final phase of $12 \mathrm{GeV}$ Upgrade to be completed in October \\
2013 & $12 \mathrm{GeV}$ Commissioning of the new Accelerator begins in November \\
2013 & One-pass beam at 2.2 GeV by the end of the year \\
2014 & First beam to Hall A at greater than $6 \mathrm{GeV}$ in February \\
2014 & First beam to Hall D at greater than $10 \mathrm{GeV}$ in May \\
2016 & First beam to Halls B and C at $11 \mathrm{GeV}$ \\
\hline \hline
\end{tabular}

\subsection{ACCELERATOR OVERVIEW}

This section provides a high level description of the CEBAF accelerator which is shown schematically in Fig. 1. The present machine is designed to accelerate electrons to $6 \mathrm{GeV}$ by recirculating the beam five times through two $1497 \mathrm{MHz}$ superconducting RF (SRF) linear accelerators. Each of the 200 meter long linacs (see Fig. 2) consist of twenty cryomodules containing eight 5-cell cavities operating at a superfluid liquid helium temperature of $2 \mathrm{~K}$. A photograph of one of the 5-cell cavities showing the elliptical cell shape is shown in Fig. 3. Each cavity has an active length of $0.5 \mathrm{~m}$. The average accelerating gradient for the cavities is $7.5 \mathrm{MV} / \mathrm{m}$ resulting in an energy increase of $600 \mathrm{MeV}$ per linac or $1200 \mathrm{MeV}$ per pass.

The beam starts in the upper left corner of the diagram in Fig. 1 at the Injector's polarized electron source, where three interleaved $499 \mathrm{MHz}$ lasers are used to create the RF micropulse structure of the electron beam. The three lasers are at the 


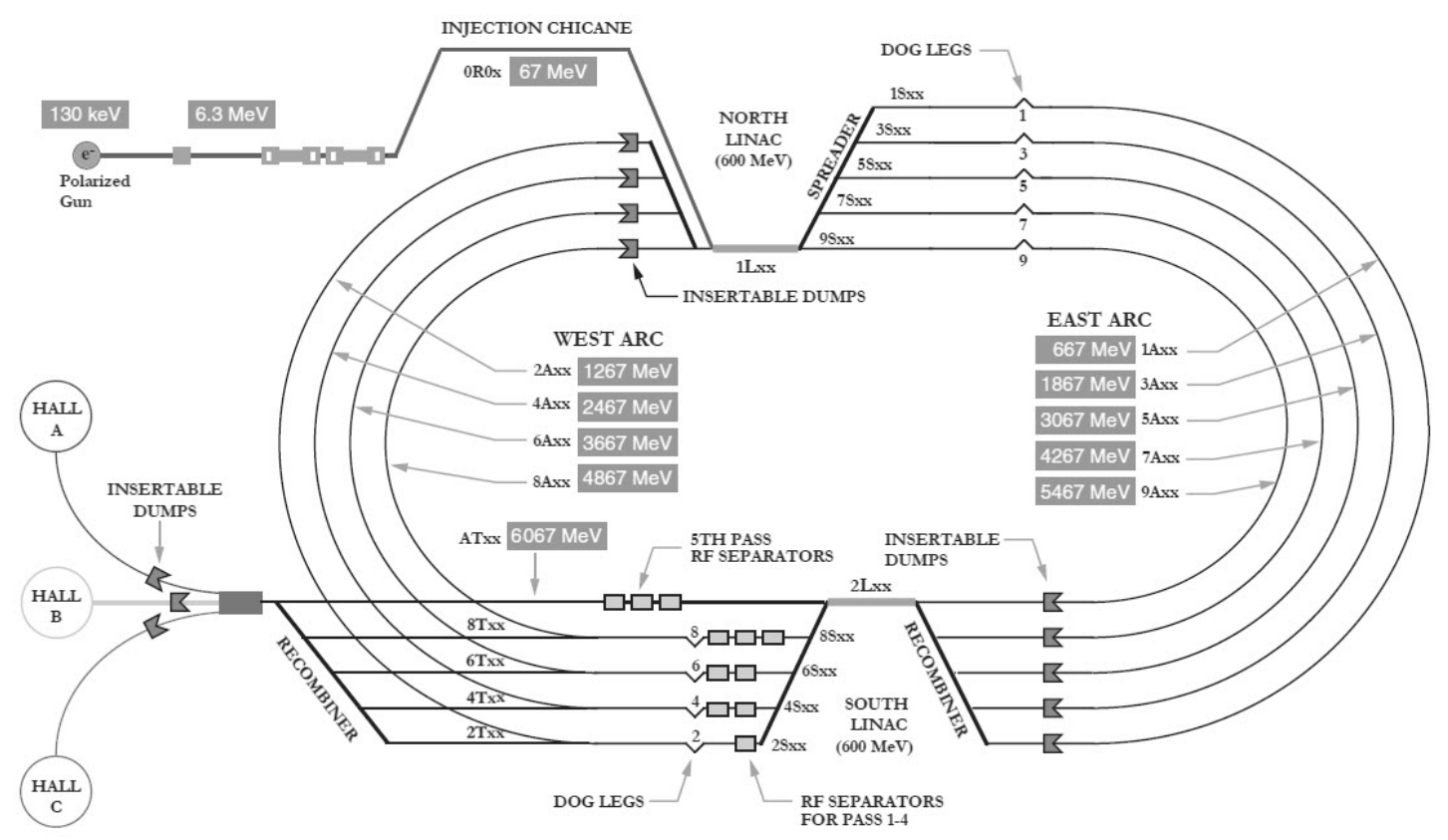

FIG. 1. A schematic layout of the CEBAF accelerator showing the characteristic racetrack design (Drawing courtesy of Tom Oren).

third subharmonic of the SRF cavities, separated in phase by $120^{\circ}$ and are used to independently control the beam current to each of the three experimental Halls A,B, and C. The collinear lasers are focussed onto a small wafer of Gallium Arsenide, a semiconductor cathode material with high quantum efficiency, to create the beam of electrons. The cathode is held at a potential of $130 \mathrm{kV}$, hence the initial beam energy of $130 \mathrm{keV}$.

The next segment of the Injector provides longitudinal bunching of the electron beam and acceleration to a relativistic energy of $6.3 \mathrm{MeV}$ using a warm copper graded-beta cavity followed by two 5-cell SRF cavities. The beam is then accelerated to the final injection energy of $67 \mathrm{MeV}$ using two standard eight-cavity SRF cryomodules. The final segment of the Injector contains a set of quadrupoles used for matching the beam envelope to the next segment and a chicane section for transporting the beam to the entrance of the North Linac.

The beam is accelerated to $667 \mathrm{MeV}$ after the first transit through the twenty cryomodules of the North Linac. Each of the one hundred and sixty SRF cavities are phased so that their peak electric field coincides with the arrival of the electron bunch. Between each linac cryomodule is a quadrupole for focussing the beam. The quads 


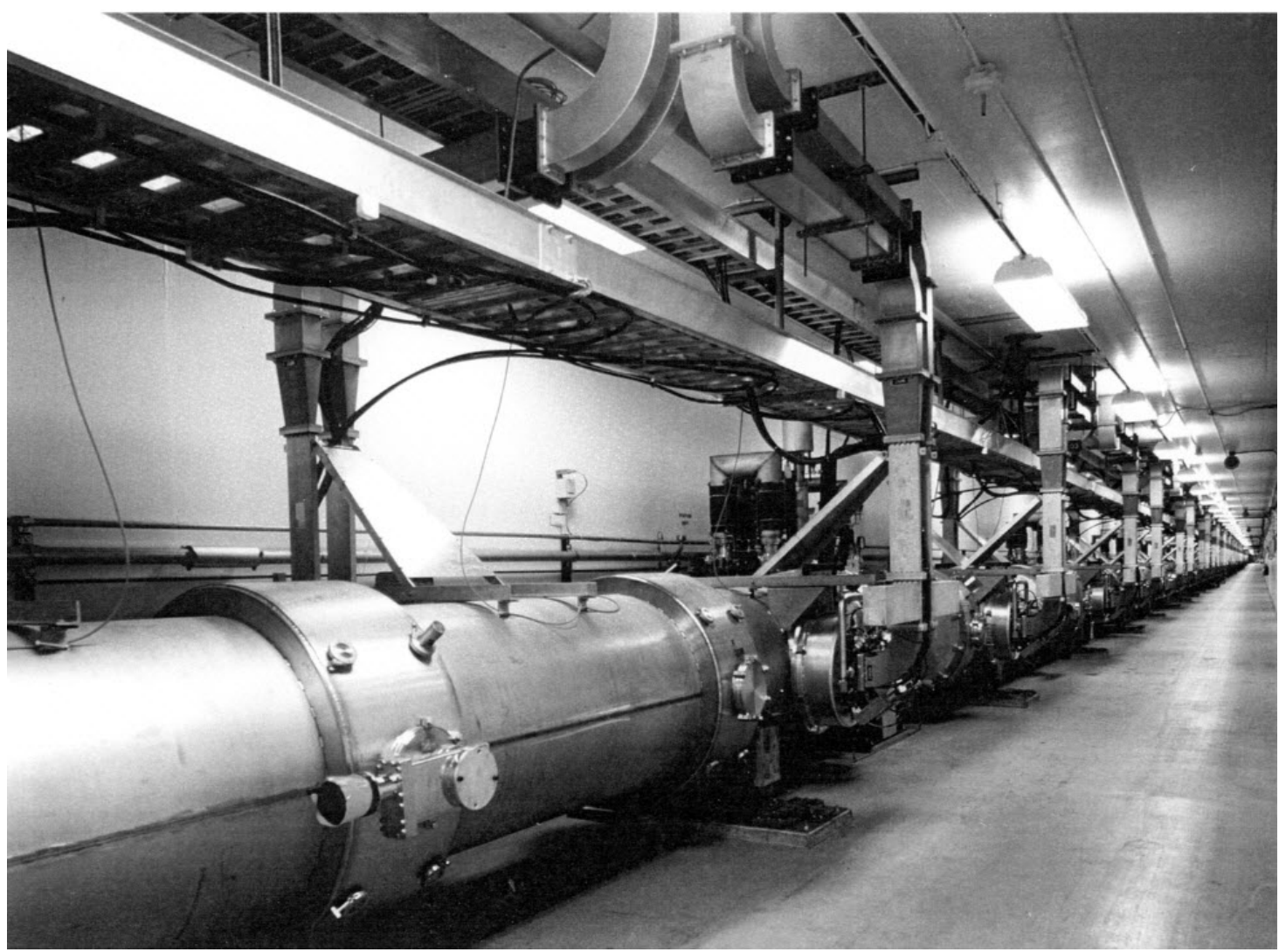

FIG. 2. A photograph of one of the CEBAF linear accelerators. Each of the linac's twenty cryomodules operate at $2 \mathrm{~K}$ and provide an average energy gain of $30 \mathrm{MeV}$ (Photo from JLAB archive).

are arranged in an alternating gradient structure with one period of oscillation of the beam envelope for the first pass beam occurring every third cryomodule. This is referred to as a $120^{\circ} \mathrm{FODO}$ lattice. The quadrupole strength increases monotonically as the beam gains energy in the linac.

At the end of the North Linac is the East Spreader beamline which changes the vertical elevation of the beam. The first pass beam is directed towards the uppermost Arc 1 beamline. The recirculation arc bends the beam through $180^{\circ}$ and is followed by a Recombiner segment that is mirror symmetric to the Spreader. The Recombiner lowers the beam back to linac elevation to prepare for another $600 \mathrm{MeV}$ energy gain.

The Spreader/Arc/Recombiner beamline is an isochronous and achromatic transport system. In an isochronous system all electrons travel the same distance independent of energy. In an achromatic system the position and angle of the beam at the exit is independent of energy. Within the Recombiner segment are four tuning 


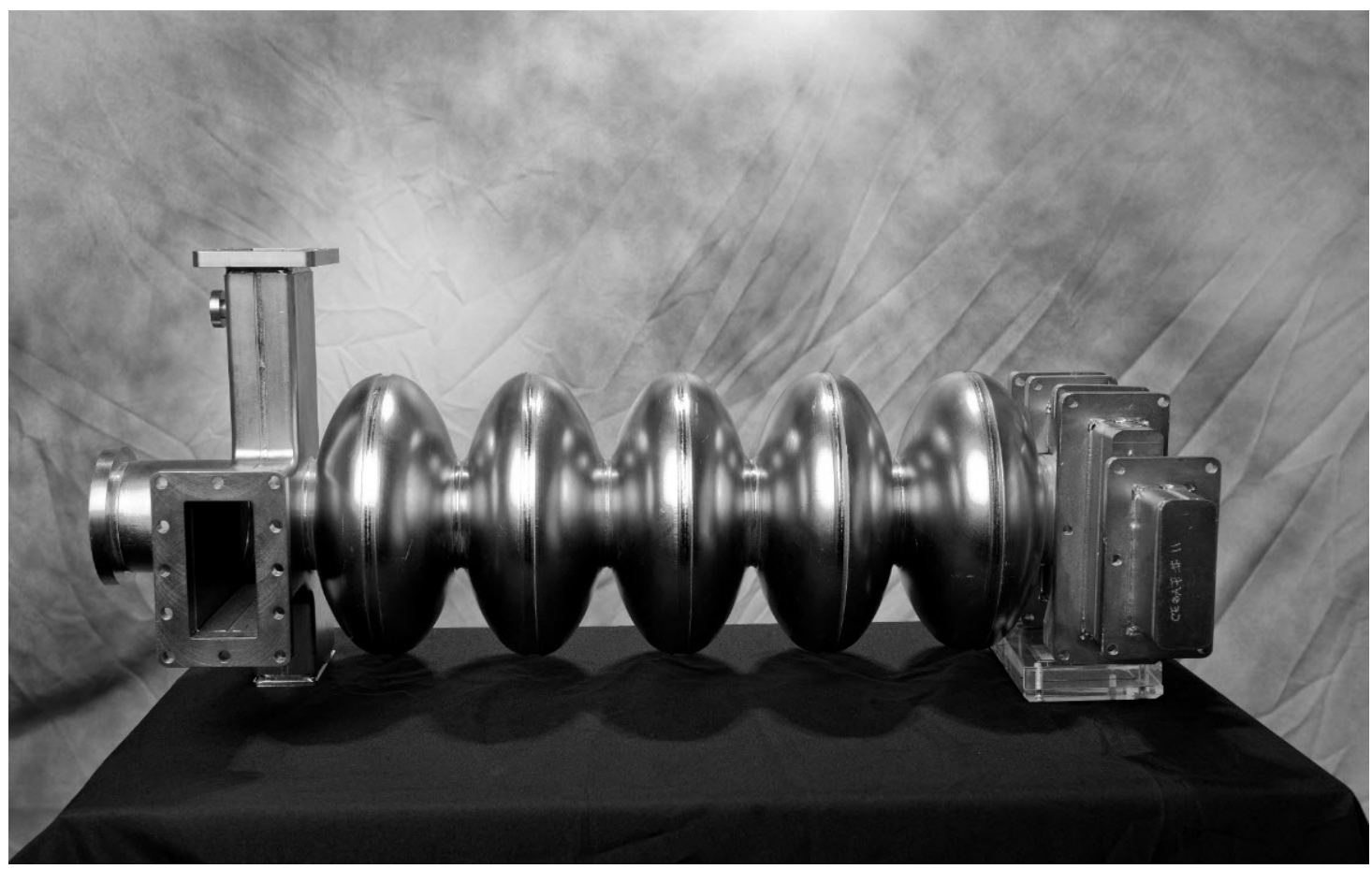

FIG. 3. A photograph of one of the CEBAF 5-cell niobium cavities. The elliptical cells have an active length of $0.5 \mathrm{~m}$ with each cavity providing an average energy gain of 3.75 MeV (Photo from JLAB archive).

quadrupoles for matching the beam envelope to the next Arc.

The beam is transported through the South Linac for the first time and accelerated to $1267 \mathrm{MeV}$. The SRF cavity phasing and quadrupole configuration of the South Linac is identical to what was mentioned above for the North Linac. At the end of the linac is the West Spreader which changes the vertical elevation of the beam for transport into the Arc 2 and West Recombiner beamlines. The optical properties of this Spreader/Arc/Recombiner section are identical to the Arc 1 section.

Between the end of the West Spreader and the start of the West Arc is a beam extraction system consisting of horizontally deflecting RF Separator cavities [1] and pairs of septa magnets. The cavities operate at $499 \mathrm{MHz}$ which is the same frequency as the three-laser system in the Injector. If Halls A, B, or C require beam at this first pass energy the cavities are turned on and phased to provide peak deflection to the left for that hall's electron bunch. The other two beams will be deflected to the right at half the angle due to the $120^{\circ}$ phase relationship between the bunches. Beyond the separator cavities the beams drift apart and then enter the first Septa magnet which 


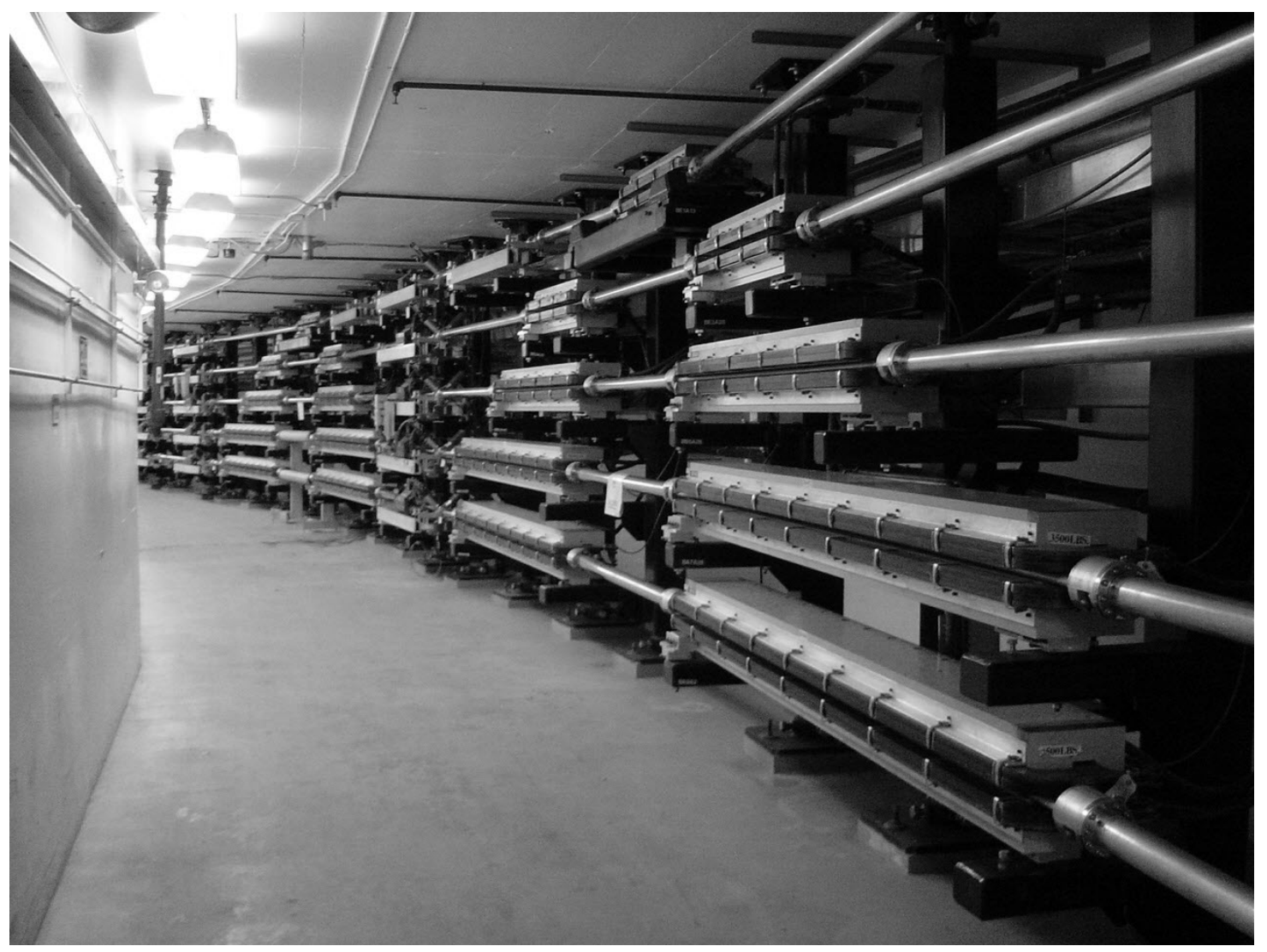

FIG. 4. A photograph of a section of the East Arc. The lowest energy Arc 1 beamline is at the top with Arcs 3,5,7 and 9 stacked below (Photo from JLAB archive).

has a strong horizontal dipole field on the left of the septa and zero field on the right. This dipole field provides an additional horizontal kick for the extracted beam. The beams continue to separate as they drift towards the second Septa which provides a final kick of the extracted beam towards the Beam Switchyard Recombiner section of the machine.

The recirculated beams are reinjected into the North Linac for another $600 \mathrm{MeV}$ energy gain. To ensure that the arrival time of the second pass beam is at the crest of the RF wave a three magnet chicane system in the preceding Arc is used to change the distance that the beam travels. These so-called Dogleg magnets are capable of changing the path length by $1 \mathrm{~cm}$ or 18 degrees of the $1497 \mathrm{MHz} \mathrm{RF}$ wave.

As the beam leaves the North Linac for the second time it once again transits the East Spreader but this time at a higher energy. The total vertical deflection is $0.5 \mathrm{~m}$ less than the first time through resulting in beam transport into Arc 3. The mirror 


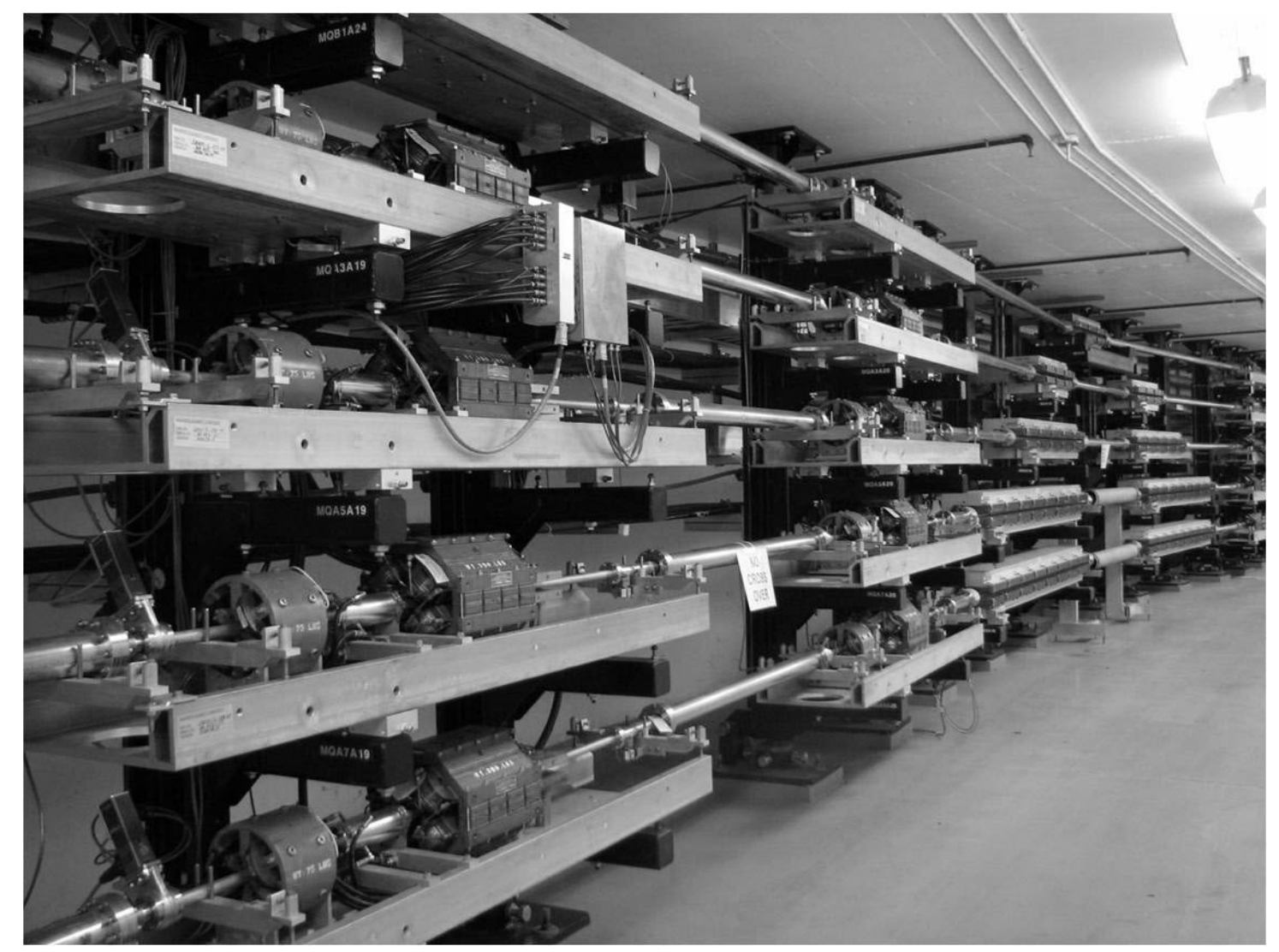

FIG. 5. A photograph showing stacks of magnet assemblies in the East Arc. The order of elements is sextupole, BPM, quadrupole and corrector (Photo from JLAB archive).

symmetric Recombiner returns the beam to the south linac elevation for another energy gain of $600 \mathrm{MeV}$. The optimal arrival time for the second pass beam in the South Linac is controlled by the Arc 3 Dogleg magnets.

This pattern repeats for each pass around the accelerator with beams extracted as necessary to meet the Nuclear Physics program. The final 5-pass energy, which is $6067 \mathrm{MeV}$, can be shared by all three user facilities through the use of a vertically deflecting extraction system in the Beam Switchyard Recombiner beamline. The separator cavities are phased to allow the Hall B beam to pass through on zerocrossing while the Hall A beam is kicked up and the Hall $\mathrm{C}$ beam is kicked down. A pair of vertical Septa are used to increase the separation.

The Beam Switchyard Recombiner returns the extracted beams to the proper elevation for transport into one of the three hall's beamlines. A stacked pair of 
horizontally deflecting magnets called the Lambertson kicks the beams towards the proper hall for that pass. The Hall A beam enters the upper channel and is deflected to the right. The Hall $\mathrm{C}$ beam enters the lower channel and is deflected left. The Hall B beam enters the center channel and is undeflected.

The beam for Hall A is transported to the target through a right hand bend of 37.5 degrees and terminates in a $1 \mathrm{MW}$ beam dump. The hall has two polarimiters for measuring the polarization of the beam. For most experiments the hall uses an electron spectrometer and a hadron spectrometer to conduct their research.

The Hall B target is approximately $3.4 \mathrm{~m}$ above the linac elevation. The beam is transported to the hall through a pair of antisymmetric bends and a ramp section to arrive on target. The hall can perform tagged photon experiments by sending the beam through a thin radiator and then dumping the electron beam vertically into a beam dump. The photon beam then hits the target and the electron that created the photon is tagged in the electron spectrometer. The hall can also turn off the tagging system, remove the radiator and take electrons directly onto the target. The spectrometer for this hall is called the CEBAF Large Acceptance Spectrometer and surrounds the entire target for nearly $4 \pi$ steradians of acceptance.

The Hall $\mathrm{C}$ transport line is mirror symmetric to Hall A with a 37.5 degree bend to the left. The hall also has two polarimiters for measuring the polarization. While the initial physics program did use a pair of spectrometers similar to Hall A this hall has more recently been used to field more specialized experiments. 


\section{CHAPTER 3}

\section{THEORY}

In this chapter the theoretical basis for the research presented in this thesis is developed. The fundamental characteristics of the different types of magnets that are used in charged particle transport is presented in section 3.1 [2]. To track the general path of the beam from one part of the accelerator to the next we need a mathematical framework to represent the different types of magnets that the beam encounters. The components that make up a beamline are collectively referred to as the lattice. A standard matrix formalism [3] for representing the linear optics of particle accelerators is presented in section 3.2. A simple model to indicate how nonlinear effects can be included in the tracking of a beam through the lattice is shown in section 3.3. The chapter concludes with an introduction to Chebyshev polynomials [4] which are used as the expansion basis for fitting the spectra that result from the transverse modulation of the beam.

\subsection{MAGNETS}

Accelerators are designed to transport charged particles along a carefully prescribed path which is referred to as the design trajectory. Beams of electrons tend to diverge from one another due to the mutual repulsive Coulomb forces that act between them. Electromagnetic fields are used to focus the electrons back to the design trajectory. These restoring forces originate from the classic Lorentz forces given by

$$
\vec{F}=e[\vec{E}+\vec{v} \times \vec{B}]=\frac{d(\gamma m \vec{v})}{d t},
$$

where $\vec{E}$ is the electric field, $\vec{B}$ is the magnetic induction, $\vec{v}$ is the velocity, and $\gamma m \vec{v}$ is the relativistic momentum. When the beam is at non-relativistic energies both electric and magnetic fields may be used to guide the beam. At relativistic energies we have $\vec{F}=e[\vec{E}+c \vec{B}]$, so a magnetic field of strength $B=1$ Tesla has the equivalent effect of an electric field of strength $E=300 \mathrm{MV} / \mathrm{m}$. Conventional designs of magnets can easily reach a field strength of $1 \mathrm{~T}$. Achieving electric field 
strengths of $100 \mathrm{MV} / \mathrm{m}$ is however impractical so magnetic fields are generally used in relativistic beam transport.

Setting the electric field to zero in Eq. (1) and using the standard relationship for the cross product

$$
\vec{v} \times \vec{B}=\left|\begin{array}{ccc}
i & j & k \\
v_{x} & v_{y} & v_{z} \\
B_{x} & B_{y} & B_{z}
\end{array}\right|,
$$

we can derive the three cartesian components for the magnetic part of the Lorentz force. They are given by

$$
\begin{aligned}
& \frac{d\left(\gamma m v_{x}\right)}{d t}=e\left[v_{y} B_{z}-v_{z} B_{y}\right], \\
& \frac{d\left(\gamma m v_{y}\right)}{d t}=e\left[v_{z} B_{x}-v_{x} B_{z}\right], \\
& \frac{d\left(\gamma m v_{z}\right)}{d t}=e\left[v_{x} B_{y}-v_{y} B_{x}\right] .
\end{aligned}
$$

The length of a typical accelerator magnet is much larger than it's transverse aperture or bore radius. This means that the longitudinal field along the $z$ direction is much smaller than the transverse fields along the $x$ and $y$ directions. This hard edge model is a good approximation for real magnets. Setting $B_{z}$ to zero in Eq. (3) and rearranging terms gives an expression for the change in transverse momentum

$$
\Delta\left(\gamma m v_{x}\right)=|e|\left[v_{z} B_{y}\right] \Delta t
$$

Rewriting the parameter $\Delta t$ in terms of the beam's longitudinal velocity $v_{z}$ and the length of the magnet $L$ we can write

$$
\begin{gathered}
\Delta\left(\gamma m v_{x}\right)=|e|\left[v_{z} B_{y}\right] \frac{L}{v_{z}}, \\
\Delta v_{x}=\frac{|e|}{\gamma m} B_{y} L .
\end{gathered}
$$

The longitudinal velocity is related to the transverse velocity by $v_{x}=v_{z} \tan \theta$. Since $v_{z}>>v_{x}$ we can use the small angle approximation and write

$$
\Delta \theta=\frac{\Delta v_{x}}{v_{z}}=\frac{|e|}{\gamma m v_{z}} B_{y} L .
$$

The change in angle is proportional to the strength of the transverse magnetic field times the length of the magnet and inversely proportional to the beam's longitudinal momentum. 
Using the most practical units for accelerator physics we have the numerical expression

$$
\Delta \theta(\mathrm{rad})=2.9979 \times 10^{-4} \frac{B_{y}(\mathrm{G}) L(\mathrm{~cm})}{p_{z}(\mathrm{MeV} / \mathrm{c})} .
$$

Returning to Eq. (9) and rearranging terms we have

$$
\frac{\Delta \theta}{L}=\frac{|e|}{\gamma m v_{z}} B_{y}=\frac{|e|}{p_{z}} B_{y}
$$

Introducing the momentum rigidity $B \rho=p_{z} / e$ and writing the left side in terms of the radius of curvature we have

$$
\frac{1}{R}=\frac{1}{B \rho} B_{y}
$$

Since the transverse size of the electron beam is much smaller than the radius of curvature we can expand the magnetic field about the nominal trajectory in the power series as

$$
B_{y}(x)=B_{0}+\frac{d B_{y}}{d x} x+\frac{1}{2 !} \frac{d^{2} B_{y}}{d x^{2}} x^{2}+\frac{1}{3 !} \frac{d^{3} B_{y}}{d x^{3}} x^{3}+\cdots .
$$

Multiplying by $1 / B \rho$

$$
\frac{1}{B \rho} B_{y}(x)=\frac{1}{B \rho} B_{0}+\frac{1}{B \rho} \frac{d B_{y}}{d x} x+\frac{1}{B \rho} \frac{1}{2 !} \frac{d^{2} B_{y}}{d x^{2}} x^{2}+\frac{1}{B \rho} \frac{1}{3 !} \frac{d^{3} B_{y}}{d x^{3}} x^{3} \cdots,
$$

and introducing some constants to simplify the expression we can write

$$
\frac{1}{B \rho} B_{y}(x)=\frac{1}{R}+k x+\frac{1}{2 !} m x^{2}+\frac{1}{3 !} o x^{3}+\cdots .
$$

The magnetic field near the beam can be regarded as a sum of multipoles, each of which has a different effect on the beam. The first term is the dipole field responsible for beam steering. The next term is the quadrupole field used for focussing the beam and together with the dipole term comprise the linear optics of the accelerator. The third term is the sextupole field which is typically used for chromatic compensation. The last term is the octupole field which is used for the compensation of field errors in a lattice. The higher order multipoles can also be attributed to field errors in the dipole and quadrupole magnets which need to be minimized through the magnet design process.

The CEBAF accelerator consists of over 2800 magnets including dipoles, quadrupoles, correctors, and sextupoles. In the rest of this section the theoretical basis for these iron dominated magnets is developed. Following is a derivation of 
the functional form of the magnetic field within the gap for each magnet type. This result is then used to show how the strength of that field depends on the current in the coils and the gap between the poles.

For static electric fields the differential form of Ampere's Law is

$$
\nabla \times \vec{H}=\vec{J}
$$

The current density $\vec{J}$ is zero within the vacuum space of the beam pipe where we are interested in knowing the field and so

$$
\nabla \times \vec{H}=0
$$

From vector calculus it is always true that $\nabla \times \nabla \phi=0$ for an arbitrary scalar potential $\phi$. Therefore, Eq. (17) is automatically satisfied by allowing $\vec{H}$ to be written in terms of a scalar potential,

$$
\vec{H}=\nabla \phi
$$

For convenience we use the magnetic flux density $\vec{B}=\mu_{0} \vec{H}$, where $\mu_{0}$ is the permeability of free space. Re-scaling the potential according to $\Phi(x, y)=\mu_{0} \phi(x, y)$ gives the expression

$$
\vec{B}=\nabla \Phi
$$

Using Maxwell's equation $\nabla \cdot \vec{B}=0$ with this equation gives Laplace's equation

$$
\nabla^{2} \Phi=0
$$

To determine the shape of the transverse field everywhere within the magnet we write the general expression

$$
B_{y}(x, y)=G_{y}(x)+f(y)
$$

The first term is the field in the $y$ direction as we move along the $x$ axis. The second term is an unknown function that captures the dependence of the field on the $y$ coordinate. The potential is then written as

$$
\Phi(x, y)=\int B_{y} d y=G_{y}(x) y+\int f(y) d y
$$

Using Laplace's equation in two dimensions to find $f(y)$ we have

$$
\nabla^{2} \Phi=\frac{\partial^{2} \Phi}{\partial x^{2}}+\frac{\partial^{2} \Phi}{\partial y^{2}}=\frac{d^{2} G_{y}(x)}{d x^{2}} z+\frac{d f(z)}{d z}=0 .
$$



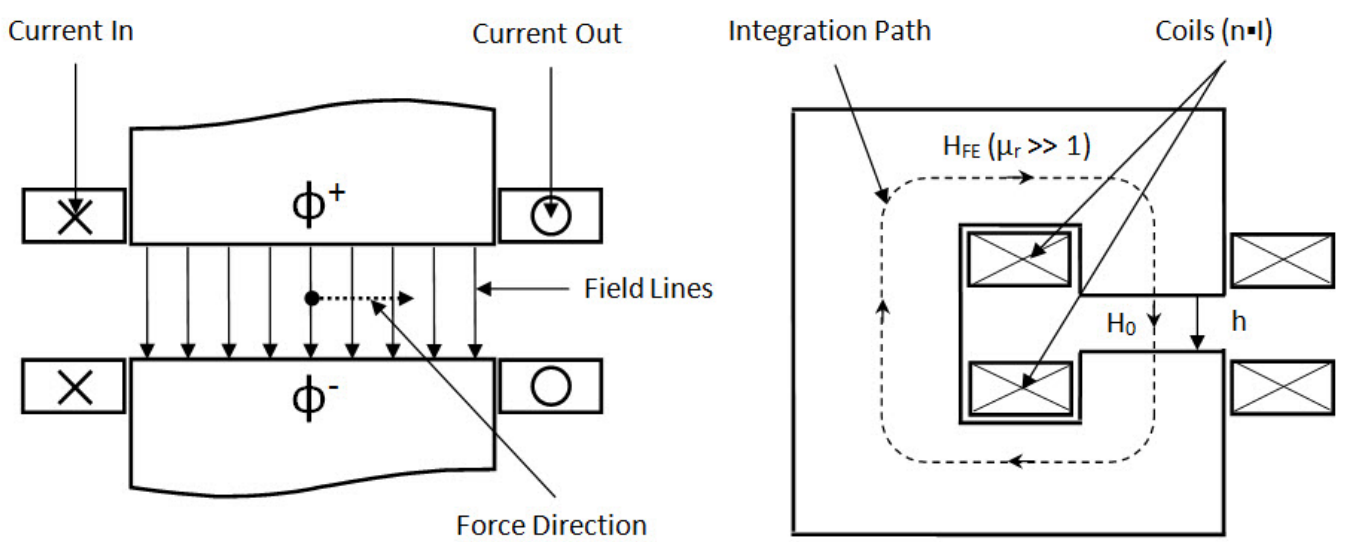

FIG. 6. Schematic diagram of an ideal dipole (left) and an example of a C-shaped dipole showing the path of integration used to determine the magnetic field (right).

Rearranging the right hand side and integrating the equation gives the proper expression for

$$
f(y)=-\int \frac{d^{2} G_{y}(x)}{d x^{2}} y d y=-\frac{1}{2} \frac{d^{2} G_{y}(x)}{d x^{2}} y^{2} .
$$

Substituting this result into Eq. (25) and integrating one more time yields the general expression for the two-dimensional potential

$$
\Phi(x, y)=\int B_{y} d y=G_{y}(x) y-\frac{1}{6} \frac{d^{2} G_{y}(x)}{d x^{2}} y^{3} .
$$

By inspecting the individual terms in the multipole expansion of Eq. (15) we can choose the proper values of $G_{y}(x)$ and $d^{2} G_{y}(x) / d x^{2}$ for a dipole, quadrupole, sextupole, and octupole. This calulation will not work above octupole since the Laplacian is no longer exactly zero when $d^{4} G / d x^{4} \neq 0$.

The field distribution everywhere within the magnet aperture is then calculated by taking the gradient of the potential. This calculation provides the so-called upright or normal multipoles with the magnetic fields oriented vertically along the horizontal centerline. There are also skew multipoles where the magnetic field is horizontal along the horizontal centerline. These are determined by rotating a dipole by $90^{\circ}$, a quadrupole by $45^{\circ}$, and a sextupole by $30^{\circ}$ which can be visualized by simply rotating Figs. 6, 7, and 8 by the prescribed amount.

First we look at a dipole magnet which is shown in Fig. 6. From the multipole expansion of Eq. (15) we see that the dipole field is constant in the $y$ direction and 
the gradient is zero. Putting this result into Eq. (25) we have

$$
\Phi(x, y)=B_{0} y
$$

The fields come from the derivative of the potential and are given by

$$
\begin{gathered}
B_{x}(x, y)=\frac{\partial}{\partial x}\left(B_{0} y\right)=0 \\
B_{y}(x, y)=\frac{\partial}{\partial y}\left(B_{0} y\right)=B_{0} .
\end{gathered}
$$

As is shown in the left hand side of Fig. 6, the ideal dipole provides a force in the midplane which steers the electron beam. CEBAF uses horizontally bending dipoles, such as shown here, in the Arcs and vertically bending dipoles in the Spreaders and Recombiners which are just rotated by $90^{\circ}$.

Looking at the multipole expansion again we see that a quadrupole field is zero in the center of the magnet and increases linearly as we move along the $x$ axis. The aperture of a quadrupole is shown schematically in Fig. 7. Using this result in Eq. (25) we write

$$
\Phi(x, y)=g x y
$$

where $g=\frac{d B_{y}}{d x}$. It follows that the field of a quadrupole is given by

$$
\begin{aligned}
& B_{x}(x, y)=\frac{\partial}{\partial x}(g x y)=g y, \\
& B_{y}(x, y)=\frac{\partial}{\partial y}(g x y)=g x .
\end{aligned}
$$

As is shown in Fig. 7, the fields within a quadrupole focus the beam in one plane while defocussing the beam in the other plane.

Looking at the multipole expansion we see that a sextupole field is zero in the center but grows quadratically as we move along the $x$ axis. The fields within the aperture of a sextupole are shown in Fig. 8. Using this result in Eq. (25) we write

$$
\Phi(x, y)=\frac{1}{2} g^{\prime} x^{2} y-\frac{1}{6} g^{\prime} y^{3}=\frac{g^{\prime}}{2}\left(x^{2} y-\frac{y^{3}}{3}\right),
$$

where $g^{\prime}=d^{2} B_{y} / d x^{2}$. The sextupole fields come once again from the derivatives of the potential and are given by

$$
\begin{array}{r}
B_{x}(x, y)=\frac{g^{\prime}}{2} \frac{\partial}{\partial x}\left(x^{2} y-\frac{y^{3}}{3}\right)=g^{\prime} x y \\
B_{y}(x, y)=\frac{g^{\prime}}{2} \frac{\partial}{\partial y}\left(x^{2} y-\frac{y^{3}}{3}\right)=\frac{g^{\prime}}{2 !}\left(x^{2}-y^{2}\right) .
\end{array}
$$




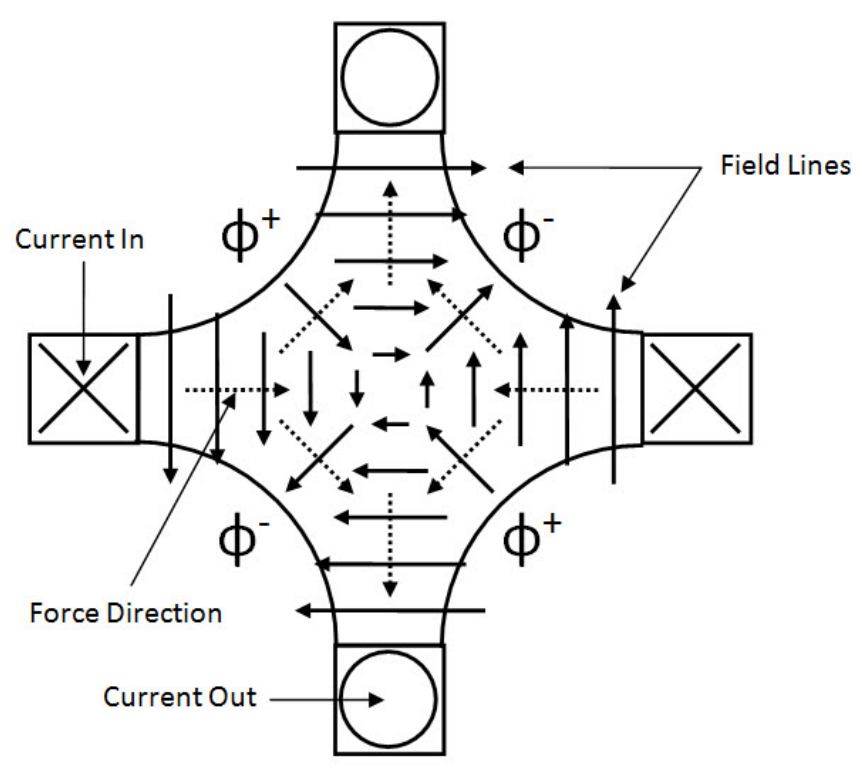

FIG. 7. A detailed drawing of the beam aperture of a quadrupole showing the direction of current flow, the potential for each pole, the field lines within the aperture and the direction of force for an electron.

Inspecting the octupole term in the multipole expansion we see that the field is zero at the center and grows cubically as we move along the $x$ axis. It follows that the potential for a normal octupole is given by

$$
\Phi(x, y)=\frac{1}{6} g^{\prime \prime} x^{3} y-\frac{1}{6} g^{\prime \prime} x y^{3}=\frac{g^{\prime \prime}}{6}\left(x^{3} y-x y^{3}\right)
$$

where $g^{\prime \prime}=d^{3} B_{y} / d x^{3}$. The resulting magnetic fields are then

$$
\begin{aligned}
& B_{x}(x, y)=\frac{g^{\prime \prime}}{6} \frac{\partial}{\partial x}\left(x^{3} y-x y^{3}\right)=\frac{g^{\prime \prime}}{3 !}\left(3 x^{2} y-y^{3}\right) \\
& B_{y}(x, y)=\frac{g^{\prime \prime}}{6} \frac{\partial}{\partial y}\left(x^{3} y-x y^{3}\right)=\frac{g^{\prime \prime}}{3 !}\left(x^{3}-3 x y^{2}\right) .
\end{aligned}
$$

The functional form of the dipole and quadrupole fields are linear and uncoupled while the sextupole and octupole fields are nonlinear and coupled.

Finally for this section, we use Ampere's circuital equation to determine the field dependence for the three types of magnets as a function of the current in the coils and the gap between the poles. The integral form of Ampere's Law is given by

$$
\oint \vec{H} \cdot d \vec{s}=I_{t o t a l}
$$




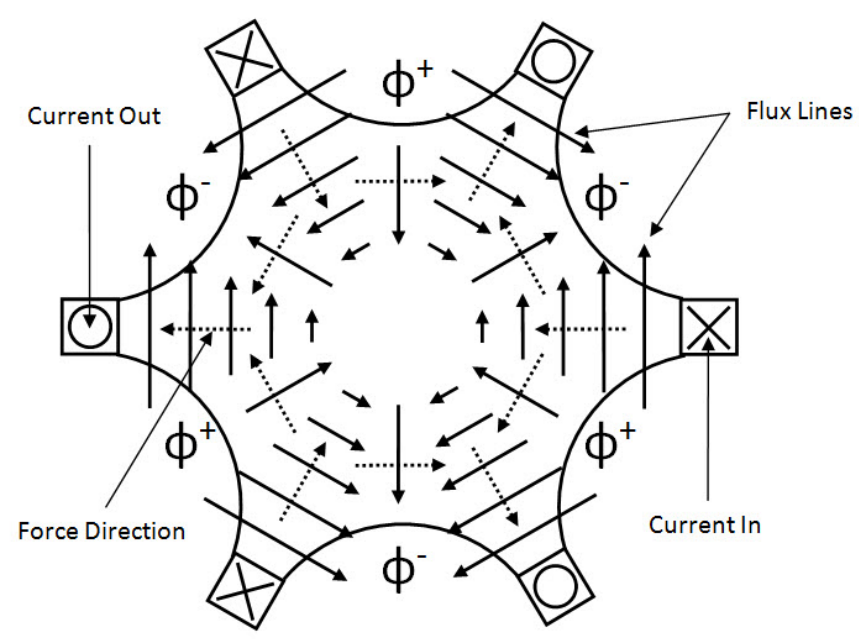

FIG. 8. A detailed drawing of the beam aperture of a sextupole showing the direction of current flow, the potential for each pole, the field lines within the aperture and the direction of force for an electron.

where $\vec{H}$ is the magnetic field and $d \vec{s}$ is the differential path around a closed loop that encircles the total current. For $n$ conductors we can write $I_{\text {total }}=n I$. For the path shown in the dipole schematic of Fig. 6 we have

$$
\oint \vec{H} \cdot d \vec{s}=H_{F e} l_{F e}+H_{0} h=n I .
$$

The permeability within the iron, $\mu_{F e}$, is related to the permeability of free space, $\mu_{0}$, by $\mu_{r}=\mu_{F e} / \mu_{0}$ which is much greater than 1 . It follows that

$$
H_{F e} l_{F e}+H_{0} h \approx H_{0} h=n I .
$$

Using $B_{0}=\mu_{0} H_{0}$ we obtain the expression for the field of a dipole magnet

$$
B_{0}=\frac{\mu_{0} n I}{h}
$$

The ideal dipole field is constant along the midplane, increases linearly with the total current $n I$ and is inversely proportional to the gap between the poles $h$.

Next we consider the quadrupole magnet which is shown in Fig. 10. The path of integration can be split into three distinct parts. First we have the path within the steel which has already been shown to be small as compared to the path in the gap. Second we have the path along the $x$ axis where the field is always perpendicular to the path where $B \cdot d s$ is zero. Finally we have the segment from the origin to the 


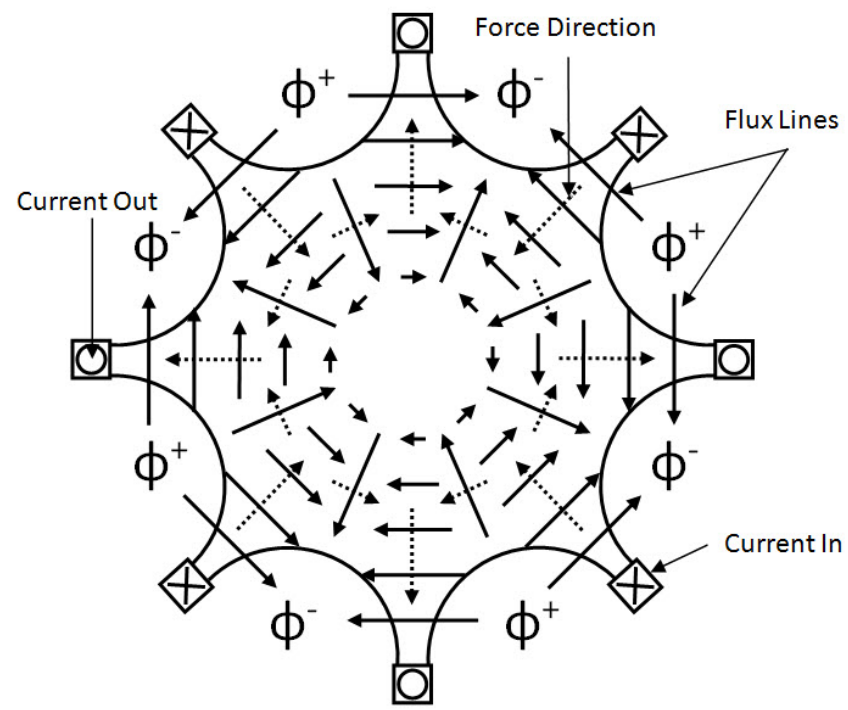

FIG. 9. A detailed drawing of the beam aperture of an octupole showing the direction of current flow, the potential for each pole, the field lines within the aperture and the direction of force for an electron.

pole face which is the only part of the integral that contributes to the determination of the field. Looking at the right side of Fig. 10 we have

$$
H_{0}=\frac{g}{\mu_{0}} \sqrt{x^{2}+y^{2}}=\frac{g}{\mu_{0}} r .
$$

Performing the integral for the only relevant part of the path we have

$$
\int_{0}^{a} H_{0} d r=\frac{g}{\mu_{0}} \int_{0}^{a} r d r=\frac{g}{\mu_{0}} \frac{a^{2}}{2}=n I .
$$

The relationship for the gradient is then

$$
g=\frac{2 \mu_{0} n I}{a^{2}} .
$$

Using $g=\partial B / \partial x$ and integrating yields the expression for the field

$$
B_{y}=\frac{2 \mu_{0} n I x}{a^{2}} .
$$

The ideal quadrupole field depends linearly on the position $x$ in the midplane, scales linearly with the total current $n I$ and is inversely proportional to the square of the pole radius $a$.

Finally we consider the sextupole magnet which is shown in Fig. 11. Once again the only contribution to the integral is from the origin to the pole tip radius. Using 

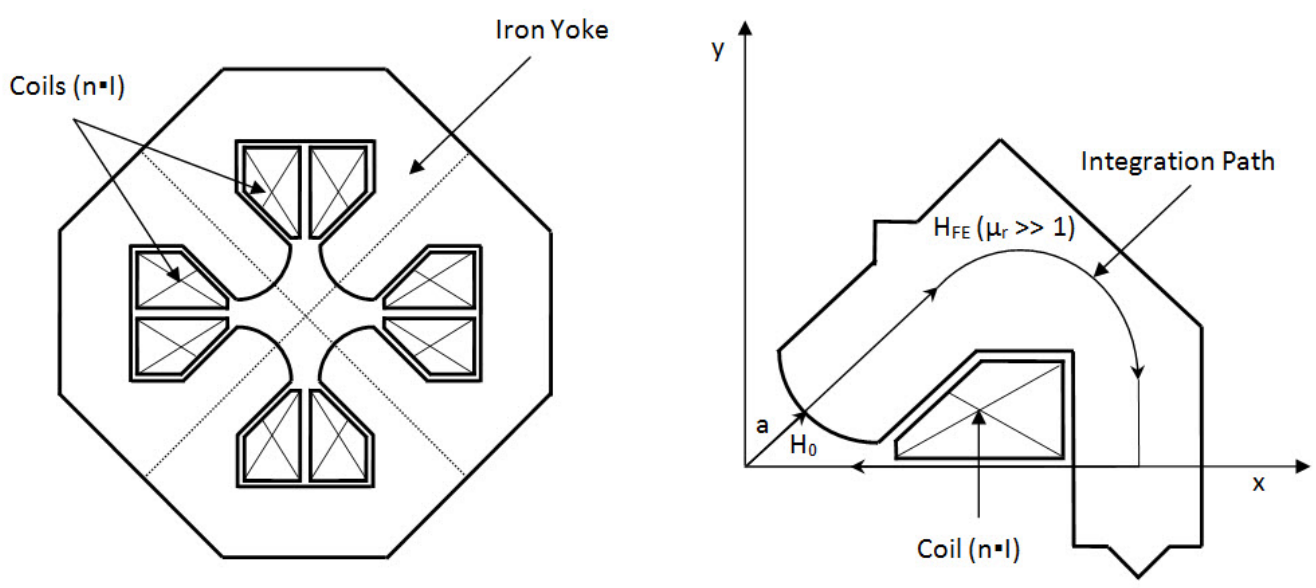

FIG. 10. Schematic diagram of a quadrupole (left) and a detailed picture of one of the poles showing the path of integration used to determine the magnetic field (right).

the $y$-component of the field for a sextupole and $H_{0}=B_{0} / \mu_{0}$ we can write

$$
\int_{0}^{a} H_{0} d r=\frac{g^{\prime}}{\mu_{0}} \int_{0}^{a}\left(x^{2}-y^{2}\right) d r .
$$

The pole is oriented at $30^{\circ}$ above the midplane. Substituting this in the above equation and solving the integral yields

$$
\begin{gathered}
\int_{0}^{a} H_{0} d r=\frac{g^{\prime}}{\mu_{0}}\left[\int_{0}^{a} r^{2} \cos ^{2}\left(30^{\circ}\right) d r-\int_{0}^{a} r^{2} \sin ^{2}\left(30^{\circ}\right) d r\right] \\
\int_{0}^{a} H_{0} d r=\frac{g^{\prime}}{2 \mu_{0}} \int_{0}^{a} r^{2} d r \\
\frac{g^{\prime}}{6 \mu_{0}} a^{3}=n I
\end{gathered}
$$

Solving for $g^{\prime}$ we have

$$
g^{\prime}=\frac{6 \mu_{0} n I}{a^{3}}
$$

Using $g^{\prime}=\partial^{2} B / \partial x^{2}$ and integrating twice yields the expression for the field

$$
B_{y}=\frac{3 \mu_{0} n I x^{2}}{a^{3}} .
$$

The ideal sextupole field depends quadratically on the position $x$ in the midplane, scales linearly with the total current $n I$ and is inversely proportional to the cube of the pole radius $a$. 

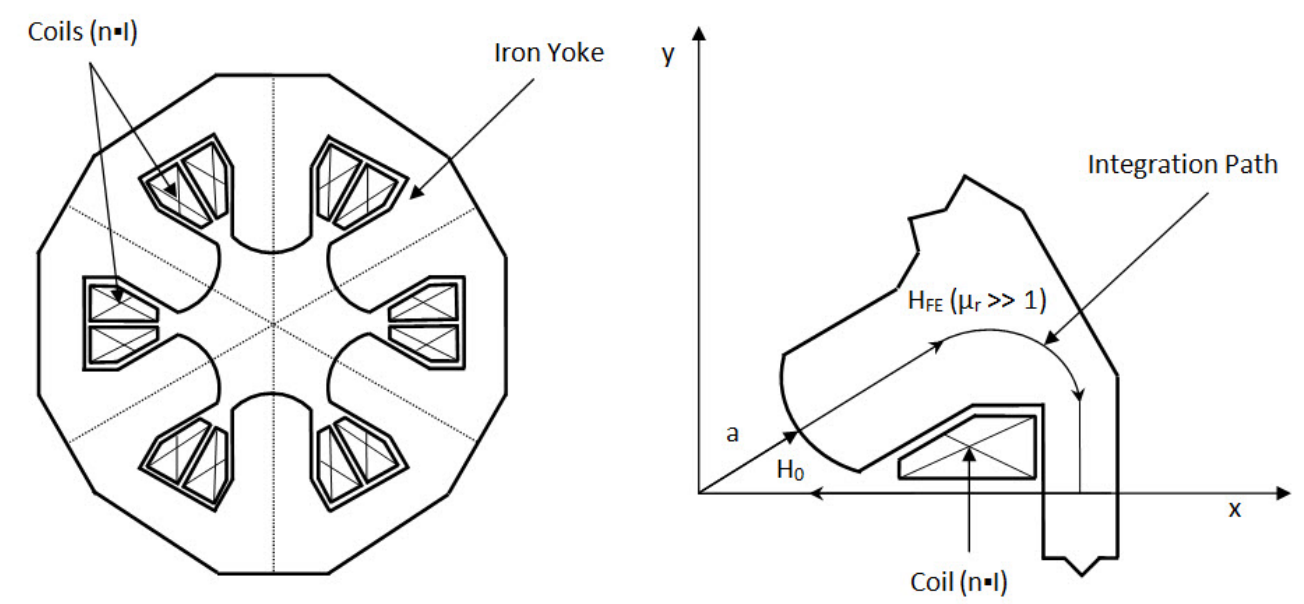

FIG. 11. Schematic diagram of a sextupole (left) and a detailed picture of one of the poles showing the path of integration used to determine the magnetic field (right).

\subsection{BEAM OPTICS}

In this section the matrix formalism for describing the linear optics of the accelerator is developed. As was shown in Eq. (15) the magnetic field near the beam can be expanded into a series of multipole strengths with the first two being identified as the linear optics. The beam is guided along the reference orbit by the dipole fields and oscillates about that trajectory in response to the restoring forces of the quadrupoles around the machine. The motion is referred to as betatron oscillations after they were first observed by Kerst and Serber [5] in an accelerator called the Betatron in 1941. The general equations that form the basis of linear optics are the second-order differential Hill's [6] equations. We write them in two dimensions as

$$
\begin{aligned}
x^{\prime \prime}(s)+\left(\frac{1}{R^{2}(s)}-k(s)\right) x(s) & =\frac{1}{R(s)} \frac{\Delta p}{p}, \\
y^{\prime \prime}(s)+k(s) y(s) & =0
\end{aligned}
$$

where $x$ and $y$ are the transverse coordinates, $s$ is the longitudinal beam-following coordinate, $R$ is the radius of curvature within a dipole field, $k$ is the quadrupole focussing strength and $\Delta p / p$ captures the relative error in the beam momentum. The $x$-plane was selected as the principal bending plane for this discussion. Considering the case for $\Delta p=0$, two-dimensional matrices for quadrupoles, drifts and dipoles in 
the presence of a monochromatic beam can be developed. The general equations are now homogenous and can be solved analytically. The equations of motion reduce to

$$
\begin{aligned}
x^{\prime \prime}(s)+\left(\frac{1}{R^{2}(s)}-k(s)\right) x(s) & =0, \\
y^{\prime \prime}(s)+k(s) y(s) & =0 .
\end{aligned}
$$

The hard-edge model for transverse magnetic fields of the last section is used in what follows. In other words, the fields are constant within the magnets and we also assume that the magnets are absent of any multipole effects higher than quadrupole.

We first solve the equations of motion for quadrupoles. There is no dipole term and so $1 / R=0$ in Eq. (52). The equations of motion are then written as

$$
\begin{aligned}
& x^{\prime \prime}(s)-k(s) x(s)=0, \\
& y^{\prime \prime}(s)+k(s) y(s)=0 .
\end{aligned}
$$

For $k>0$ we have a quadrupole that defocusses in the $x$-plane and focusses in the $y$-plane. We then solve the equations of motion for the position and angle with the initial beam conditions $x_{0}, y_{0}, x_{0}^{\prime}$, and $y_{0}^{\prime}$. The resulting equations are

$$
\begin{aligned}
x(s) & =x_{0} \cosh \sqrt{k} s+\frac{x_{0}^{\prime}}{\sqrt{k}} \sinh \sqrt{k} s, \\
x^{\prime}(s) & =x_{0} \sqrt{k} \sinh \sqrt{k} s+x_{0}^{\prime} \cosh \sqrt{k} s, \\
y(s) & =y_{0} \cos \sqrt{k} s+\frac{y_{0}^{\prime}}{\sqrt{k}} \sin \sqrt{k} s, \\
y^{\prime}(s) & =y_{0} \sqrt{k} \sin \sqrt{k} s+y_{0}^{\prime} \cos \sqrt{k} s .
\end{aligned}
$$

Letting $\phi=\sqrt{k} s$ and arranging the solution in a matrix form we have

$$
\left[\begin{array}{c}
x(s) \\
x^{\prime}(s) \\
y(s) \\
y^{\prime}(s)
\end{array}\right]=\left[\begin{array}{cccc}
\cosh \phi & \frac{1}{\sqrt{k}} \sinh \phi & 0 & 0 \\
\sqrt{k} \sinh \phi & \cosh \phi & 0 & 0 \\
0 & 0 & \cos \phi & \frac{1}{\sqrt{k}} \sin \phi \\
0 & 0 & -\sqrt{k} \sin \phi & \cos \phi
\end{array}\right]\left[\begin{array}{c}
x_{0} \\
x_{0}^{\prime} \\
y_{0} \\
y_{0}^{\prime}
\end{array}\right] .
$$

For $k<0$ we have a quadrupole that focusses in the $x$-plane and defocusses in the $y$-plane. We then solve the equations of motion with the same initial conditions as 
before. The resulting equations are now written as

$$
\begin{aligned}
x(s) & =x_{0} \cos \sqrt{|k|} s+\frac{x_{0}^{\prime}}{\sqrt{|k|}} \sin \sqrt{|k|} s, \\
x^{\prime}(s) & =x_{0} \sqrt{|k|} \sin \sqrt{|k|} s+x_{0}^{\prime} \cos \sqrt{|k|} s, \\
y(s) & =y_{0} \cosh \sqrt{|k|} s+\frac{y_{0}^{\prime}}{\sqrt{|k|}} \sinh \sqrt{|k|} s, \\
y^{\prime}(s) & =y_{0} \sqrt{|k|} \sinh \sqrt{|k|} s+y_{0}^{\prime} \cosh \sqrt{|k|} s .
\end{aligned}
$$

Assuming now that $\phi=\sqrt{|k|} s$ the solution in matrix form is

$$
\left[\begin{array}{c}
x(s) \\
x^{\prime}(s) \\
y(s) \\
y^{\prime}(s)
\end{array}\right]=\left[\begin{array}{cccc}
\cos \phi & \frac{1}{\sqrt{|k|}} \sin \phi & 0 & 0 \\
-\sqrt{|k|} \sin \phi & \cos \phi & 0 & 0 \\
0 & 0 & \cosh \phi & \frac{1}{\sqrt{|k|}} \sinh \phi \\
0 & 0 & \sqrt{|k|} \sinh \phi & \cosh \phi
\end{array}\right]\left[\begin{array}{c}
x_{0} \\
x_{0}^{\prime} \\
y_{0} \\
y_{0}^{\prime}
\end{array}\right] .
$$

Next we consider the solution for a field-free region or drift. In this case we have $1 / R=0$ and $k=0$ and Eqs. (54) and (55) are written as

$$
\begin{aligned}
& x^{\prime \prime}(s)=0, \\
& y^{\prime \prime}(s)=0 .
\end{aligned}
$$

These are readily solved to give the equations of motion for a drift:

$$
\begin{aligned}
x(s) & =x_{0}+s x_{0}^{\prime}, \\
x^{\prime}(s) & =x_{0}^{\prime} \\
y(s) & =y_{0}+s y_{0}^{\prime} \\
y^{\prime}(s) & =y_{0}^{\prime} .
\end{aligned}
$$

The solutions in matrix form for a field-free region are given by

$$
\left[\begin{array}{l}
x(s) \\
x^{\prime}(s) \\
y(s) \\
y^{\prime}(s)
\end{array}\right]=\left[\begin{array}{llll}
1 & s & 0 & 0 \\
0 & 1 & 0 & 0 \\
0 & 0 & 1 & s \\
0 & 0 & 0 & 1
\end{array}\right]\left[\begin{array}{l}
x_{0} \\
x_{0}^{\prime} \\
y_{0} \\
y_{0}^{\prime}
\end{array}\right]
$$


For a pure dipole with no edge focussing we can set $k=0$ in Eqs. (54) and (55) and write

$$
\begin{aligned}
x^{\prime \prime}(s)+\left(\frac{1}{R^{2}(s)}\right) x(s) & =0, \\
y^{\prime \prime}(s) & =0 .
\end{aligned}
$$

The solutions for these equations are

$$
\begin{aligned}
x(s) & =x_{0} \cos \frac{s}{R}+x_{0}^{\prime} R \sin \frac{s}{R}, \\
x^{\prime}(s) & =\frac{-x_{0}}{R} \sin \frac{s}{R}+x_{0}^{\prime} \cos \frac{s}{R}, \\
y(s) & =y_{0}+s y_{0}^{\prime}, \\
y^{\prime}(s) & =y_{0}^{\prime},
\end{aligned}
$$

and in matrix form we have

$$
\left[\begin{array}{c}
x(s) \\
x^{\prime}(s) \\
y(s) \\
y^{\prime}(s)
\end{array}\right]=\left[\begin{array}{cccc}
\cos \frac{s}{R} & R \sin \frac{s}{R} & 0 & 0 \\
-R \sin \frac{s}{R} & \cos \frac{s}{R} & 0 & 0 \\
0 & 0 & 1 & s \\
0 & 0 & 0 & 1
\end{array}\right]\left[\begin{array}{c}
x_{0} \\
x_{0}^{\prime} \\
y_{0} \\
y_{0}^{\prime}
\end{array}\right] .
$$

These matrices make it easy to track particles through the lattice. Any arbitrary sequence of linear beamline elements can be represented by a series of transfer matrices. The matrix for the whole beamline is equal to the product of the individual matrices. For the example in Fig. 12 we have

$$
M_{\text {lattice }}=M_{1} \cdot M_{2} \cdot M_{3} \cdot M_{4} \cdot M_{5} \cdot M_{6} \cdot M_{7} \cdot M_{8} \cdot M_{9}
$$

One can construct more realistic magnet models using this formalism. For example placing a dipole matrix between two focussing matrices provides a dipole with edge effects. As was shown in section 3.1 the field of a sextupole is coupled in the transverse plane so linear matrices cannot be used. To account for the complexities of real magnets and include edge-effects and nonlinearities one must use simulation code. Two software packages were used in this thesis. For predicting the multipole content of dipole magnets as a function of the beam trajectory we used the TOSCA package from Vector-Fields [7] which is described in section 5.3. For tracking particles through 


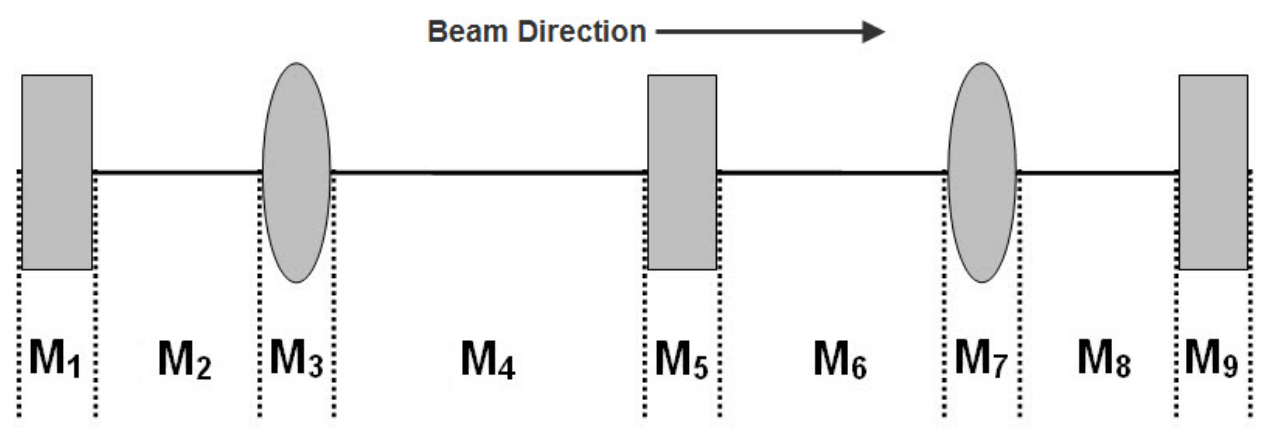

FIG. 12. An arbitrary sequence of beamline elements.

the lattice we used a program called elegant [8] which is described in section 5.4. The beam-based measurements and analysis naturally include all of these effects.

\subsection{ANALYTICAL MODEL}

A simple model of zero-length elements can be used to demonstrate how two different AC kicker frequencies will propagate and mix across a nonlinear lattice. The beamline lattice is shown in Fig. 13 and consists of two kicker magnets, a sextupole to provide the nonlinear forces and a beam position monitor to measure the transverse location of the beam relative to the reference orbit.

Transfer matrices are used to represent the beamline between the elements of the model as was shown in section 3.2. The segment between the two AC kickers is represented by the matrix $L$, the segment between the second $\mathrm{AC}$ kicker and the

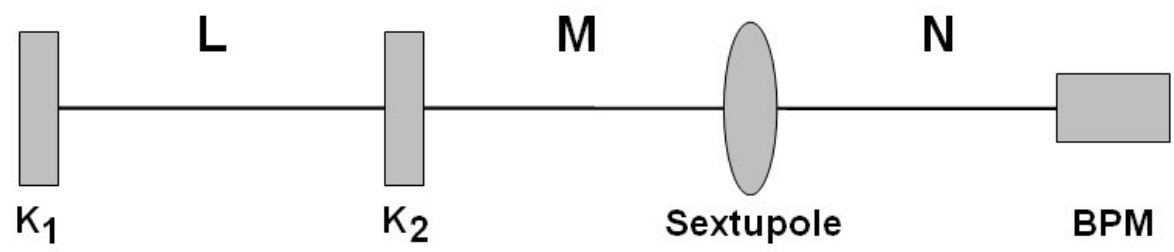

FIG. 13. A simple model of beamline elements for demonstrating how two distinct frequencies of a simultaneously modulated electron beam will mix due to the nonlinear fields of a sextupole. 
sextupole is represented by matrix $M$ and the last segment from the sextupole to the beam position monitor is represented by matrix $N$.

Modulation in the horizontal mid-plane for each of the kickers will be assumed for the development of this simple model. As the beam transports through the system a subscript for the relevant element is appended to the position and angle to keep track of where we are in the lattice. The beam position and angle entering any element will be noted with a minus sign in the subscript while the angle and position leaving a beamline element will be noted with a plus sign in the subscript.

For beam entering the system a set of initial conditions with zero position and angle is chosen which gives at the entrance of the first kicker

$$
X_{K_{1}-}=X_{K_{1-}}^{\prime}=0
$$

An AC modulation with amplitude $A_{1}$ and frequency $\omega_{1}$ is applied to the first kicker. Since the elements are assumed to have zero length the position at the exit of the kicker is unchanged. The AC modulation changes the angle as the beam leaves the kicker. At the exit of the first kicker we have

$$
\begin{gathered}
X_{K_{1}+}=0, \\
X_{K_{1}+}^{\prime}=A_{1} \cos \omega_{1} t .
\end{gathered}
$$

The transfer matrix $L$ is used to transport the beam from the exit of the first kicker to the entrance of the second kicker. The one dimensional representation is given by

$$
\left(\begin{array}{l}
X_{K_{2}-} \\
X_{K_{2}-}^{\prime}
\end{array}\right)=\left(\begin{array}{ll}
L_{11} & L_{12} \\
L_{21} & L_{22}
\end{array}\right)\left(\begin{array}{c}
X_{K_{1}+} \\
X_{K_{1}+}^{\prime}
\end{array}\right) .
$$

Substituting the expression for the position and angle at the kicker exit yields

$$
\begin{aligned}
& X_{K_{2}-}=L_{12} A_{1} \cos \omega_{1} t, \\
& X_{K_{2}-}^{\prime}=L_{22} A_{1} \cos \omega_{1} t .
\end{aligned}
$$

Now an AC modulation with amplitude $A_{2}$ and frequency $\omega_{2}$ is applied to the second kicker. The modulation adds an additional angle as the beam leaves the kicker but does not affect the beam position within the zero-length element. The position of the beam at the exit of the second kicker magnet is then given by

$$
X_{K_{2}+}=L_{12} A_{1} \cos \omega_{1} t
$$


while the angle is given by

$$
X_{K_{2}+}^{\prime}=L_{22} A_{1} \cos \omega_{1} t+A_{2} \cos \omega_{2} t
$$

The transfer matrix $M$ is used to transport the beam from the exit of the second kicker to the entrance of the sextupole. The matrix is again written as

$$
\left(\begin{array}{c}
X_{S-} \\
X_{S-}^{\prime}
\end{array}\right)=\left(\begin{array}{ll}
M_{11} & M_{12} \\
M_{21} & M_{22}
\end{array}\right)\left(\begin{array}{c}
X_{K_{2}+} \\
X_{K_{2}+}^{\prime}
\end{array}\right) .
$$

Substituting the initial conditions we have the position of the beam at the entrance of the sextupole given by

$$
\begin{gathered}
X_{S-}=M_{11} L_{12} A_{1} \cos \omega_{1} t+M_{12}\left(L_{22} A_{1} \cos \omega_{1} t+A_{2} \cos \omega_{2} t\right), \\
X_{S-}=\left(M_{11} L_{12}+M_{12} L_{22}\right) A_{1} \cos \omega_{1} t+M_{12} A_{2} \cos \omega_{2} t, \\
X_{S-}=(M L)_{12} A_{1} \cos \omega_{1} t+M_{12} A_{2} \cos \omega_{2} t .
\end{gathered}
$$

The beam angle at the entrance of the sextupole is given by

$$
\begin{gathered}
X_{S-}^{\prime}=M_{21} L_{12} A_{1} \cos \omega_{1} t+M_{22}\left(L_{22} A_{1} \cos \omega_{1} t+A_{2} \cos \omega_{2} t\right), \\
\left.X_{S-}^{\prime}=\left(M_{21} L_{12}+M_{22} L_{22}\right) A_{1} \cos \omega_{1} t+M_{22} A_{2} \cos \omega_{2} t\right), \\
X_{S-}^{\prime}=(M L)_{22} A_{1} \cos \omega_{1} t+M_{22} A_{2} \cos \omega_{2} t .
\end{gathered}
$$

As was shown in section 3.1 the magnetic field for a sextupole in the $x$ and $y$ plane is given by

$$
\begin{gathered}
B_{x}(x, y)=\frac{\delta^{2} B_{y}}{\delta x^{2}} x y \\
B_{y}(x, y)=\frac{1}{2} \frac{\delta^{2} B_{y}}{\delta x^{2}}\left(x^{2}-y^{2}\right) .
\end{gathered}
$$

For the development of this model the beam modulations are restricted to the horizontal midplane. Under these conditions $y=0$ and we only need to consider

$$
B_{y}(x, 0)=\frac{1}{2} \frac{\delta^{2} B_{y}}{\delta x^{2}}\left(x^{2}\right)=\frac{g^{\prime}}{2} x^{2},
$$

where the sextupole gradient from section 3.1 has been used. The position at the exit of the zero-length sextupole is equal to the position at the entrance while the angle now has an additional term due to the Lorentz force of the field $B_{y}$ which depends 
on the square of the position within the sextupole. The position of the beam at the exit of the sextupole is given by

$$
X_{S+}=(M L)_{12} A_{1} \cos \omega_{1} t+M_{12} A_{2} \cos \omega_{2} t
$$

while the angle is given by

$$
\begin{aligned}
X_{S+}^{\prime}= & (M L)_{22} A_{1} \cos \omega_{1} t+M_{22} A_{2} \cos \omega_{2} t \\
& +\frac{g^{\prime}}{2}\left[\left((M L)_{12} A_{1}\right)^{2} \cos ^{2} \omega_{1} t\right. \\
& +2(M L)_{12} M_{12} A_{1} A_{2} \cos \omega_{1} t \cos \omega_{2} t \\
& \left.+\left(M_{12} A_{2}\right)^{2} \cos ^{2} \omega_{1} t\right] .
\end{aligned}
$$

The expression for $X_{s+}^{\prime}$ can be rewritten using the following two trigonometric identities:

$$
\begin{gathered}
\cos A \cos B=\frac{1}{2}[\cos (A+B)+\cos (A-B)], \\
\cos ^{2} A=\frac{1}{2}[1+\cos 2 A] .
\end{gathered}
$$

The modified expression for the angle of the beam leaving the sextupole is now written as

$$
\begin{aligned}
X_{S+}^{\prime}= & (M L)_{22} A_{1} \cos \omega_{1} t+M_{22} A_{2} \cos \omega_{2} t \\
& +\frac{g^{\prime}}{2}\left[\frac{1}{2}\left((M L)_{12} A_{1}\right)^{2}\left[1+\cos 2 \omega_{1} t\right]\right. \\
& +(M L)_{12} M_{12} A_{1} A_{2}\left[\cos \left(\omega_{1}+\omega_{2}\right) t+\cos \left(\omega_{1}-\omega_{2}\right)\right] \\
& \left.+\frac{1}{2}\left(M_{12} A_{2}\right)^{2}\left[1+\cos 2 \omega_{2} t\right]\right] .
\end{aligned}
$$

So we see that the angle at the exit of the sextupole carries the fundamental frequencies of $\omega_{1}$ and $\omega_{2}$ as well as the four harmonics of $2 \omega_{1}, 2 \omega_{2},\left(\omega_{1}+\omega_{2}\right)$ and $\left(\omega_{1}-\omega_{2}\right)$.

Finally the transfer matrix $N$ is used to transport the beam from the exit of the sextupole to the beam position monitor. The matrix is once again written as

$$
\left(\begin{array}{c}
X_{B P M} \\
X_{B P M}^{\prime}
\end{array}\right)=\left(\begin{array}{ll}
N_{11} & N_{12} \\
N_{21} & N_{22}
\end{array}\right)\left(\begin{array}{c}
X_{S+} \\
X_{S+}^{\prime}
\end{array}\right) .
$$

The position of the beam at the BPM is written as

$$
\begin{aligned}
X_{B P M}= & N_{11}\left[(M L)_{12} A_{1} \cos \omega_{1} t+M_{12}^{2} A_{2} \cos \omega_{2} t\right] \\
& +\frac{g^{\prime}}{2} N_{12}\left[\frac{1}{2}\left((M L)_{12} A_{1}\right)^{2}\left[1+\cos 2 \omega_{1} t\right]\right. \\
& +(M L)_{12} M_{12} A_{1} A_{2}\left[\cos \left(\omega_{1}+\omega_{2}\right) t+\cos \left(\omega_{1}-\omega_{2}\right)\right] \\
& \left.+\frac{1}{2}\left(M_{12} A_{2}\right)^{2}\left[1+\cos 2 \omega_{2} t\right]\right]
\end{aligned}
$$


TABLE 3. Harmonic sidebands for fundamental frequencies of $1 \mathrm{~Hz}$ and $21 \mathrm{~Hz}$.

\begin{tabular}{lcc}
\hline \hline Magnet Order & Harmonic Expression & Frequency $(\mathrm{Hz})$ \\
\hline \multirow{3}{*}{ Sextupole } & $2 \omega_{1}$ & 2 \\
& $2 \omega_{2}$ & 42 \\
& $\omega_{1}+\omega_{2}$ & 20 \\
$\omega_{1}-\omega_{2}$ & 22 \\
\hline \multirow{5}{*}{ Octupole } & $3 \omega_{1}$ & 3 \\
& $3 \omega_{2}$ & 63 \\
& $\omega_{2}+2 \omega_{1}$ & 23 \\
$\omega_{2}-2 \omega_{1}$ & 19 \\
$2 \omega_{2}+\omega_{1}$ & 43 \\
$2 \omega_{2}-\omega_{1}$ & 41 \\
\hline \hline
\end{tabular}

and the angle is written as

$$
\begin{aligned}
X_{B P M}^{\prime}= & N_{21}\left[(M L)_{12} A_{1} \cos \omega_{1} t+M_{12}^{2} A_{2} \cos \omega_{2} t\right] \\
& +\frac{g^{\prime}}{2} N_{22}\left[\frac{1}{2}\left((M L)_{12} A_{1}\right)^{2}\left[1+\cos 2 \omega_{1} t\right]\right. \\
& +(M L)_{12} M_{12} A_{1} A_{2}\left[\cos \left(\omega_{1}+\omega_{2}\right) t+\cos \left(\omega_{1}-\omega_{2}\right)\right] \\
& \left.+\frac{1}{2}\left(M_{12} A_{2}\right)^{2}\left[1+\cos 2 \omega_{2} t\right]\right] .
\end{aligned}
$$

The amplitude of the primary frequencies and harmonics can now be determined at the BPM by doing Fourier analysis of the time domain fluctuations of the beam. This experiment used $\omega_{1}=1 \mathrm{~Hz}$ and $\omega_{2}=21 \mathrm{~Hz}$. The different harmonics are shown in Table 3 for a sextupole as well as what one would expect in the presence of an octupole magnet's cubic transverse position dependence.

\subsection{CHEBYSHEV FORMALISM}

The Chebyshev polynomials and their unique properties were used for the analysis of the data in this thesis, as they allow one to readily obtain an upper bound on measurement errors. Its worthwhile to review the most important properties of this remarkable class of polynomials and to explain how they can be used for precision modulation measurements such as were conducted in this research.

The defining relationship for deriving Chebyshev polynomials is given by

$$
T_{n}(\cos \theta)=\cos (n \theta)
$$


Using well known trigonometric identities we can derive the first 5 Chebyshev polynomials. For $T_{0}$ we have:

$$
\begin{aligned}
T_{0}(\cos \theta) & =\cos (0)=1 \\
T_{0}(x) & =1 .
\end{aligned}
$$

For $T_{1}$ :

$$
\begin{aligned}
T_{1}(\cos \theta) & =\cos (\theta), \\
T_{1}(x) & =x .
\end{aligned}
$$

For $T_{2}$ :

$$
\begin{aligned}
T_{2}(\cos \theta) & =\cos (2 \theta)=\cos ^{2} \theta-\sin ^{2} \theta \\
& =\cos ^{2} \theta-\left(1-\cos ^{2} \theta\right) \\
& =2 \cos ^{2} \theta-1, \\
T_{2}(x) & =2 x^{2}-1 .
\end{aligned}
$$

For $T_{3}$ :

$$
\begin{aligned}
T_{3}(\cos \theta) & =\cos (3 \theta)=\cos 2 \theta \cos \theta-\sin 2 \theta \sin \theta \\
& =\cos 2 \theta \cos \theta-2 \sin ^{2} \theta \cos \theta \\
& =\left(2 \cos ^{2} \theta-1\right) \cos \theta-2\left(1-\cos ^{2} \theta\right) \cos \theta \\
& =2 \cos ^{3} \theta-\cos \theta-2 \cos \theta+2 \cos ^{3} \theta \\
& =4 \cos ^{3} \theta-3 \cos \theta, \\
T_{3}(x) & =4 x^{3}-3 x .
\end{aligned}
$$

For $T_{4}$ :

$$
\begin{aligned}
T_{4}(\cos \theta) & =\cos (4 \theta)=\cos 2 \theta \cos 2 \theta-\sin 2 \theta \sin 2 \theta \\
& =\left(2 \cos ^{2}(\theta)-1\right)^{2}-(2 \sin \theta \cos \theta)^{2} \\
& =4 \cos ^{4} \theta-4 \cos ^{2} \theta+1-4 \sin ^{2} \theta \cos ^{2} \theta \\
& =4 \cos ^{4} \theta-4 \cos ^{2} \theta+1-4 \cos ^{2} \theta\left(\frac{1-\cos 2 \theta}{2}\right) \\
& =4 \cos ^{4} \theta-4 \cos ^{2} \theta+1-2 \cos ^{2} \theta+2 \cos ^{2} \theta \cos 2 \theta \\
& =4 \cos ^{4} \theta-6 \cos ^{2} \theta+1+2 \cos ^{2} \theta\left(2 \cos ^{2} \theta-1\right) \\
& =4 \cos ^{4} \theta-6 \cos ^{2} \theta+1+4 \cos ^{4} \theta-2 \cos ^{2} \theta \\
& =8 \cos ^{4} \theta-8 \cos ^{2} \theta+1, \\
T_{4}(x) & =8 x^{4}-8 x^{2}+1 .
\end{aligned}
$$


TABLE 4. Chebyshev Polynomials from $T_{0}(x)$ through $T_{10}(x)$.

\begin{tabular}{cc}
\hline \hline$n$ & $T_{n}(x)$ \\
\hline 0 & 1 \\
1 & $x$ \\
2 & $2 x^{2}-1$ \\
3 & $4 x^{3}-3 x$ \\
4 & $8 x^{4}-8 x^{2}+1$ \\
5 & $16 x^{5}-20 x^{3}+5$ \\
6 & $32 x^{6}-48 x^{4}+18 x^{2}-1$ \\
7 & $64 x^{7}-112 x^{5}+56 x^{3}-7 x$ \\
8 & $128 x^{8}-256 x^{6}+160 x^{4}-32 x^{2}+1$ \\
9 & $256 x^{9}-576 x^{7}+432 x^{5}-120 x^{3}+9 x$ \\
10 & $512 x^{10}-1280 x^{8}+1120 x^{6}-400 x^{4}+50 x^{2}-1$ \\
\hline \hline
\end{tabular}

The recurrence formula is apparent from the results, follows directly from the addition formula for cosines and is given by

$$
T_{n+1}(x)=2 x T_{n}(x)-T_{n-1}(x) \quad n \geq 1 .
$$

The first 11 Chebyshev polynomials appear in Table 4 [9]. A few general properties of the Chebyshev polynomials are apparent by examining the table. First, the leading power of $T_{n}(x)$ for $n \geq 1$ is $2^{n-1} x^{n}$. Second, the polynomials include only even or odd powers of $x$ depending on whether $n$ is even or odd respectively. In other words the parity of $T_{n}(x)$ is $(-1)^{n}$. Third, the polynomials are all normalized so that $T_{n}(1)=1$. By the parity argument, $T_{n}(-1)=(-1)^{n}$.

The following properties of the general Chebyshev polynomial can be verified by inspection for the cases plotted in Fig. 14. First, there are $n$ zeros for the polynomial $T_{n}(x)$ and they are all contained in the domain [-1,1]. From the defining relation shown in Eq. (109), the zeros of the polynomial are given by

$$
x_{i}=\cos \left[\frac{(2 i-1) \pi}{2 n}\right] \quad i=1,2, \ldots, n .
$$

Second, the polynomial achieves the values $\pm 1, n+1$ times, at $x$ values inside the closed interval $[-1,1]$. Third, the derivative $d T_{n} / d x$ has $n-1$ zeros interleaved with the zeros of the polynomial itself. At each of these zeros the value of the polynomial is \pm 1 and the sign of the value changes for each succeeding zero. Fourth, and most 


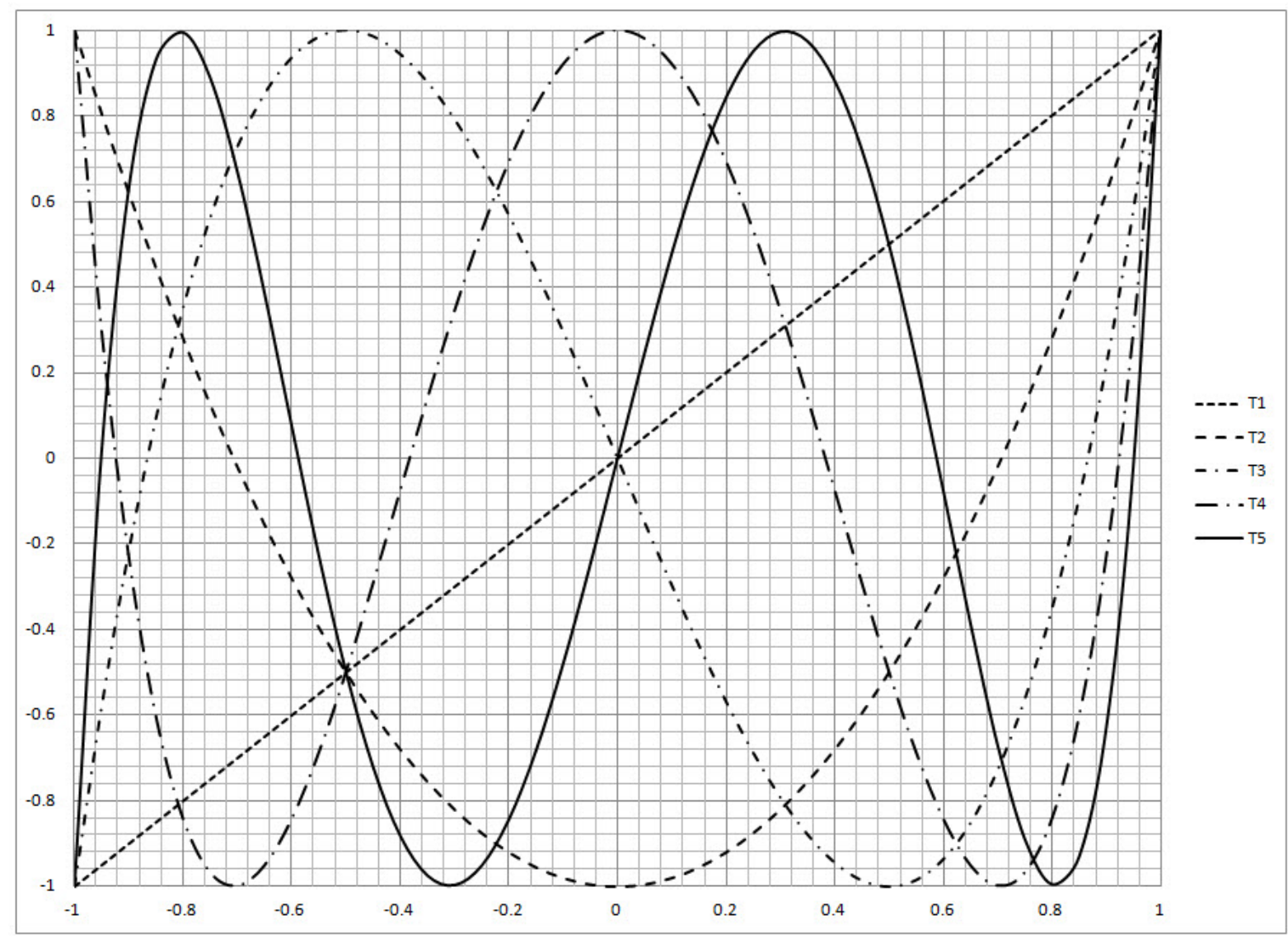

FIG. 14. A plot of Chebyshev polynomials $T_{1}(x)$ through $T_{5}(x)$.

important for error estimation, $\left|T_{n}(x)\right| \leq 1$ for all $x \in[-1,1]$. The shape of the polynomial is said to have the equal-ripple property [10], or in other words, the polynomial makes ripples of equal amplitude throughout the domain $[-1,1]$.

It should be noted that some authors [11] use the alternate normalization $T_{n}(x) / 2^{n-1}$ for the $n$th order Chebyshev polynomial. This has the benefit that the leading power of the polynomial is always one. In this thesis this normalization is not used.

Now suppose one desired to measure a real-valued function $f(x)$ whose domain is $[-1,1]$ and that, after measuring the function, one would like to represent this same function by a polynomial. A natural choice for problems of this type is to use the McLaurin-Taylor power series expansion of the function given by

$$
f(x)=f(0)+\left.\frac{d f}{d x}\right|_{0} \frac{x}{1 !}+\left.\frac{d^{2} f}{d x^{2}}\right|_{0} \frac{x^{2}}{2 !}+\cdots+\left.\frac{d^{n} f}{d x^{n}}\right|_{0} \frac{x^{n}}{n !}+\cdots
$$


In addition to the general problem of taking derivatives numerically, this representation involves values of the function only near $x=0$. When the series is truncated at a finite polynomial order $N$, the neglected terms may be quite small for values of $x$ near 0 but there is no guarantee that the representation will provide an accurate value as $x \rightarrow \pm 1$.

One solution to this deficiency is to have the polynomial representation include information about the function throughout the entire domain. A way to include this information, analogous to eigenfunction expansions in quantum mechanics, is to expand the function in modes given by sets of orthogonal polynomials. In order to pursue this line of reasoning one needs to have a way of quantifying the residual error between the function and the polynomial representation of the function. This task may be accomplished by defining suitable function-space norms. A frequently used method in statistical analysis is to require a minimum least squares error. The least squares norm of a function $g(x)$ is defined to be

$$
\|g(x)\|_{l s} \equiv\left[\int_{-1}^{1} g^{2}(x) d x\right]^{1 / 2} .
$$

Using this norm, the least squares error between a function $f(x)$ and a general $n$th order polynomial representation of the function is

$$
\operatorname{error}^{2}=\left\|f(x)-\sum_{i=0}^{N} a_{i} p_{i}(x)\right\|_{l s}^{2}=\int_{-1}^{-1}\left(f(x)-\sum_{i=0}^{N} a_{i} p_{i}(x)\right)^{2} d x,
$$

where $a_{i}$ are the polynomial expansion coefficients to be minimized and $p_{i}$ represent the general set of polynomials.

Examining Eq. (137), it is apparent that the integral may be effectively integrated when the polynomial set is orthogonal in the norm. From elementary quantum mechanics [12] it is known that the Legendre polynomial set

$$
P_{0}(x)=1 \quad P_{n}(x)=\frac{1}{2^{n} n !} \frac{d^{n}}{d x^{n}}\left(x^{2}-1\right)^{n}
$$

is orthogonal on the domain $[-1,1]$. The orthogonality relationship is given by

$$
\left\|P_{m}(x) P_{n}(x)\right\|_{l s}^{2}=\frac{2}{2 n+1} \delta_{m n},
$$

where $\delta_{m n}$ is the Kronecker delta, defined to be 1 when $m=n$ and 0 otherwise. The expression for the square of the least-squares error becomes

$$
\text { error }^{2}=\int_{-1}^{1} f^{2}(x) d x-2 \sum_{i=0}^{N} a_{i} \int_{-1}^{1} f(x) P_{i}(x) d x+\sum_{i=0}^{N} \frac{2 a_{i}^{2}}{2 i+1} .
$$


The error is minimum when the $a_{i}$ are set using the overlap integral

$$
a_{i}=\frac{2 i+1}{2} \int_{-1}^{1} f(x) P_{i}(x) d x=\frac{\left\|f(x) P_{i}(x)\right\|_{l s}^{2}}{\left\|P_{i}^{2}(x)\right\|_{l s}^{2}}
$$

The mean-square error between the function and its polynomial representation is minimized by setting the expansion coefficient to the value given by the orthonormal projection of the function onto the complete set $P_{i}(x)$.

Because $\left|P_{n}(x)\right| \leq 1$ for all $x$ in the domain [-1,1], substantial progress has been made in bounding the error of a finite polynomial expansion, as compared to a finite McLaurin-Taylor power series expansion. The absolute error of the neglected terms cannot exceed

$$
\sum_{i=n+1}^{\infty} a_{i}
$$

throughout the domain. When representations of analytical functions by such expansions are made, it is possible to estimate the infinite sum either numerically or analytically, providing an absolute bound on the neglected terms.

A related result which has a parallel for Chebyshev expansions, is that of all the polynomials with leading term $x^{n}$, the polynomial with the smallest least-squares norm is the one proportional to $P_{n}(x)$ [13]. To see this result, note that any polynomial with leading term $x^{n}$ may be written as $\sum_{i=0}^{n} a_{i} P_{i}(x)$ for some real coefficients $a_{i}$. The square of the norm is

$$
\left\|\sum_{i=0}^{n} a_{i} P_{i}(x) \sum_{j=0}^{n} a_{j} P_{j}(x)\right\|_{l s}^{2}=\sum_{i=0}^{n-1} \frac{2 a_{i}^{2}}{2 i+1}+\frac{2 a_{n}^{2}}{2 n+1} .
$$

The coefficient $a_{n}$ must be chosen to give the leading term $x^{n}$. With this choice the minimum least-square norm occurs when $a_{i}=0$ for $i=1, \cdots, n-1$.

This technique can be used to determine the function $f(x)$ by sampling points of the domain at uniformly spaced intervals and numerically computing the overlap integral to obtain the coefficients of the expansion. If the $a_{i}$ fall off fast enough with increasing $n$ the expansion can be obtained and an upper bound on the error can be found.

Another alternative is to employ a measurement technique that can naturally bound the error due to the neglected terms. Consider that the points of the domain 

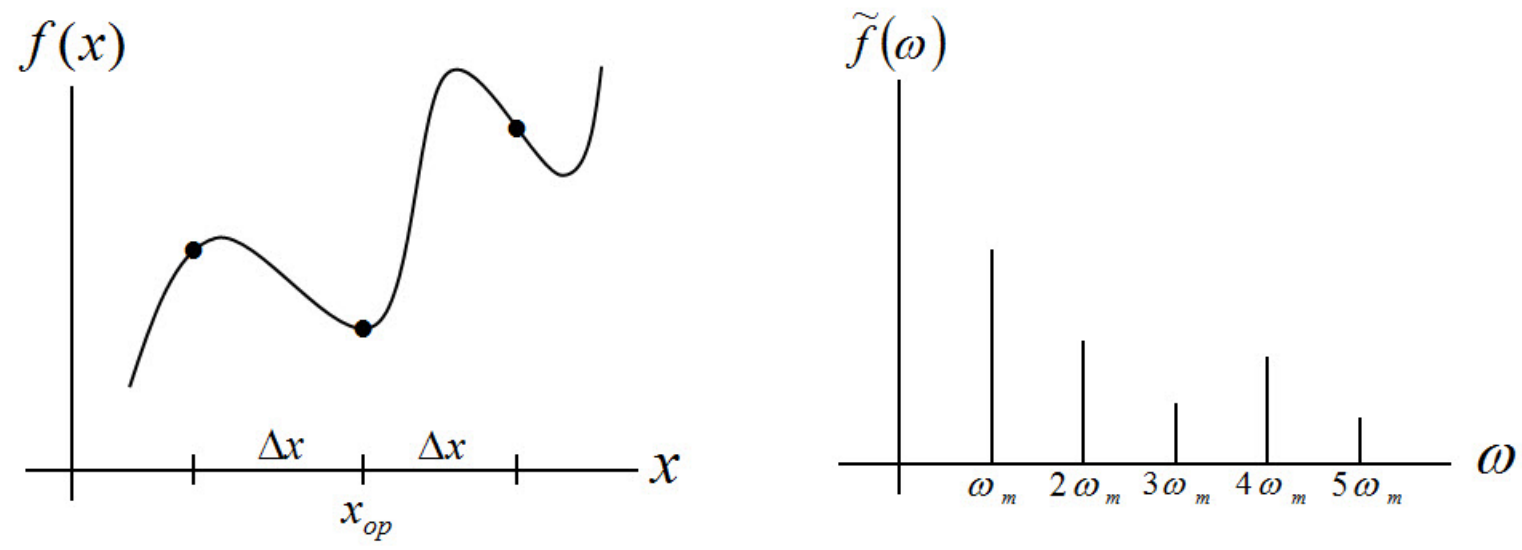

FIG. 15. The plot at the left shows an arbitrary function to fit three points using a cosine expansion. The plot at the right shows harmonic lines from the expansion.

are modulated at a certain frequency $(\omega / 2 \pi)$ as in Fig. 15 for example. The values of the modulated function $f(\cos \omega t)$ can be represented by a Fourier cosine expansion

$$
f(\cos \omega t)=f_{0}+\sum_{i=1}^{\infty} f_{i} \cos (i \omega t) .
$$

Using the standard results from Fourier theory the expansion coefficients are

$$
f_{i}=\frac{2}{\tau\left(1+\delta_{i 0}\right)} \int_{0}^{\tau} f(\cos \omega t) \cos (i \omega t) d t
$$

Now when the function to be measured is expanded in Chebyshev polynomials

$$
f(x)=\sum_{i=0}^{\infty} a_{i} T_{i}(x)
$$

the defining relation for the Chebyshev polynomials $T_{n}(\cos \theta)=\cos (n \theta)$ and the usual orthogonality of the Fourier cosine expansion yields $a_{i}=f_{i}$.

The process for measuring the function expansion in Chebyshev modes is:

1. Modulate the input of the function to be measured at a certain frequency $\omega$.

2. Detect the output of the modulation.

3. Fourier (cosine) transform the measured output.

4. Read out the expansion of the function as Chebyshev polynomials. 
The function may be highly nonlinear; the Chebyshev expansion will directly quantify the magnitude of the nonlinearity. Also note, that by the orthogonality of the Fourier series, single harmonics in the Fourier spectrum are unambiguously coupled to single Chebyshev polynomials. Response at the $i t h$ harmonic gives directly the amplitude of $T_{i}(x)$ in the function expansion and the absence of a response at the $i t h$ harmonic means that there is no $T_{i}(x)$ in the function expansion. Therefore, because the Chebyshev polynomials are bounded, the noise floor of the measured spectrum gives the maximum error possible for the measured value of the function as in the Legendre expansion above.

Two things must be considered when using this technique. First, there cannot be any significant phase delay between the modulation and the detection of the modulation. In the work reported here this is guaranteed because the beam transit time between the kickers and the BPMs is a few hundred nanoseconds which is far shorter then the modulation period. Second, to use the correspondence between Fourier and Chebyshev expansion coefficients accurately the value of the corresponding peaks of the spectrum must be determined accurately. Use of the NAFF [14] algorithm in this research maximizes the precision of the peak detection.

This method of using Fourier analysis to obtain Chebyshev expansions may be extended to two dimensions which is applicable for this thesis. For an arbitrary two-dimensional function

$$
f(x, y)=\sum_{m=0}^{\infty} \sum_{n=0}^{\infty} a_{m n} T_{m}(x) T_{n}(y)
$$

the expansion coefficients may be found using simultaneous beam modulations at two distinct frequencies with the coefficients given by

$$
a_{m n}=\frac{4}{\left(1+\delta_{m 0}\right)\left(1+\delta_{n 0}\right) \tau} \int_{0}^{\tau} f\left(\cos \omega_{1} t, \cos \omega_{2} t\right) \cos \left(m \omega_{1} t\right) \cos \left(n \omega_{2} t\right) d t .
$$

Its important for the two frequencies to be incommensurate for the lowest harmonic modes so that the identification of the spectral peaks is unambiguous. Some separation between the two frequencies is also desirable so that the sidebands don't overlap. The $1 \mathrm{~Hz}$ and $21 \mathrm{~Hz}$ frequencies used in this experiment are incommensurate as can be seen in Table 3.

The final generalization is to include arbitrary functions that are not centered at zero on average. If the modulation is over the closed two dimensional region defined by $(x, y) \in\left[x_{0}-x_{m}, x_{0}+x_{m}\right] \times\left[y_{0}-y_{m}, y_{0}+y_{m}\right]$ where $\left[x_{0}, y_{0}\right]$ is the center of the 
modulation and $\left[x_{m}, y_{m}\right]$ are the modulation amplitudes, then the expansion function is

$$
f(x, y)=\sum_{m=0}^{\infty} \sum_{n=0}^{\infty} a_{m n} T_{m}\left(\frac{x-x_{0}}{x_{m}}\right) T_{n}\left(\frac{y-y_{0}}{y_{m}}\right) .
$$

The expansion coefficients are then given by

$$
\begin{aligned}
a_{m n}= & \frac{4}{\left(1+\delta_{m 0}\right)\left(1+\delta_{n 0}\right) \tau} \times \\
& \int_{0}^{\tau} f\left(\left(x_{0}+x_{m}\right) \cos \omega_{1} t,\left(y_{0}+y_{m}\right) \cos \omega_{2} t\right) \cos \left(m \omega_{1} t\right) \cos \left(n \omega_{2} t\right) d t .
\end{aligned}
$$

An additional benefit of Chebyshev expansions is that they tend to converge very rapidly requiring fewer terms to reach the desired residue. For this reason Chebyshev polynomials are most frequently used for high-precision numerical modeling and for developing numerically efficient routines for the computation of functions.

The equal ripple property of Chebyshev polynomials provide an excellent starting point for solving the general problem of approximating a function with the minimum maximum error (minimax). Here the function space norm quantifying the error is the uniform or supremum norm given by

$$
\|f\|_{u}=\sup _{-1 \leq x \leq 1}|f(x)| .
$$

As proved by Chebyshev, of all of the polynomials with leading term $x^{n}$, the polynomial with the smallest uniform norm is $\tau_{n}(x)=T_{n}(x) / 2^{n-1}$ [15]. Another related result is that the best minimax approximation to the function $x^{n}$ by a polynomial of degree no greater than $n-1$ is given by $x^{n}-\tau_{n}(x)$ [16]. 


\section{CHAPTER 4}

\section{EXPERIMENTAL EQUIPMENT}

In this chapter the experimental equipment that was used to conduct this research is described in detail. A schematic representation of the overall hardware configuration is shown in Fig. 16.

At the lower left are the four kicker magnets for modulating the beam. Each magnet is connected to an independent current source, referred to as a trim card, in the above ground service building. A pair of function generators are used to provide the $1 \mathrm{~Hz}$ and $21 \mathrm{~Hz} \mathrm{AC}$ voltages which are used to modulate the current delivered to the magnets on the beamline. The AC voltage is also connected to the data acquisition system. The design, fabrication, measurement, and operation of the magnets will be discussed in section 4.1.

At the left are the 4-antenna Beam Position Monitors used to measure the modulation of the electron beam. Each of the eight monitors are connected to an RF module through a switching multiplexer. The multiplexer sequences through the different passes during normal operations. For this experiment the switches were fixed to the particular beamline under test. The signals from the RF module are connected to an IF module and a high speed data acquisition system, the former being part of the nominal BPM system and the latter designed for this research. The BPM system beamline components as well as the electronics are described in section 4.2.

The control room computer was used to display the BPM signals during the initial beam setup, to control the BPM calibration, and to switch the multiplexer. The electron beam macropulse structure was also controlled from the console and will be described in section 4.3.

At the upper right is the data acquisition system which records signals from all 32 BPM antennae as well as the two AC sources. The system is triggered by a beam synchronization pulse from the electron gun control system. Section 4.4 provides an overview of the Data Acquisition System and describes the sampling scheme that was used to record the $1 \mathrm{MHz}$ signal of the $\mathrm{RF}$ module and the $1 \mathrm{~Hz}$ and $21 \mathrm{~Hz}$ signals from the function generators. 


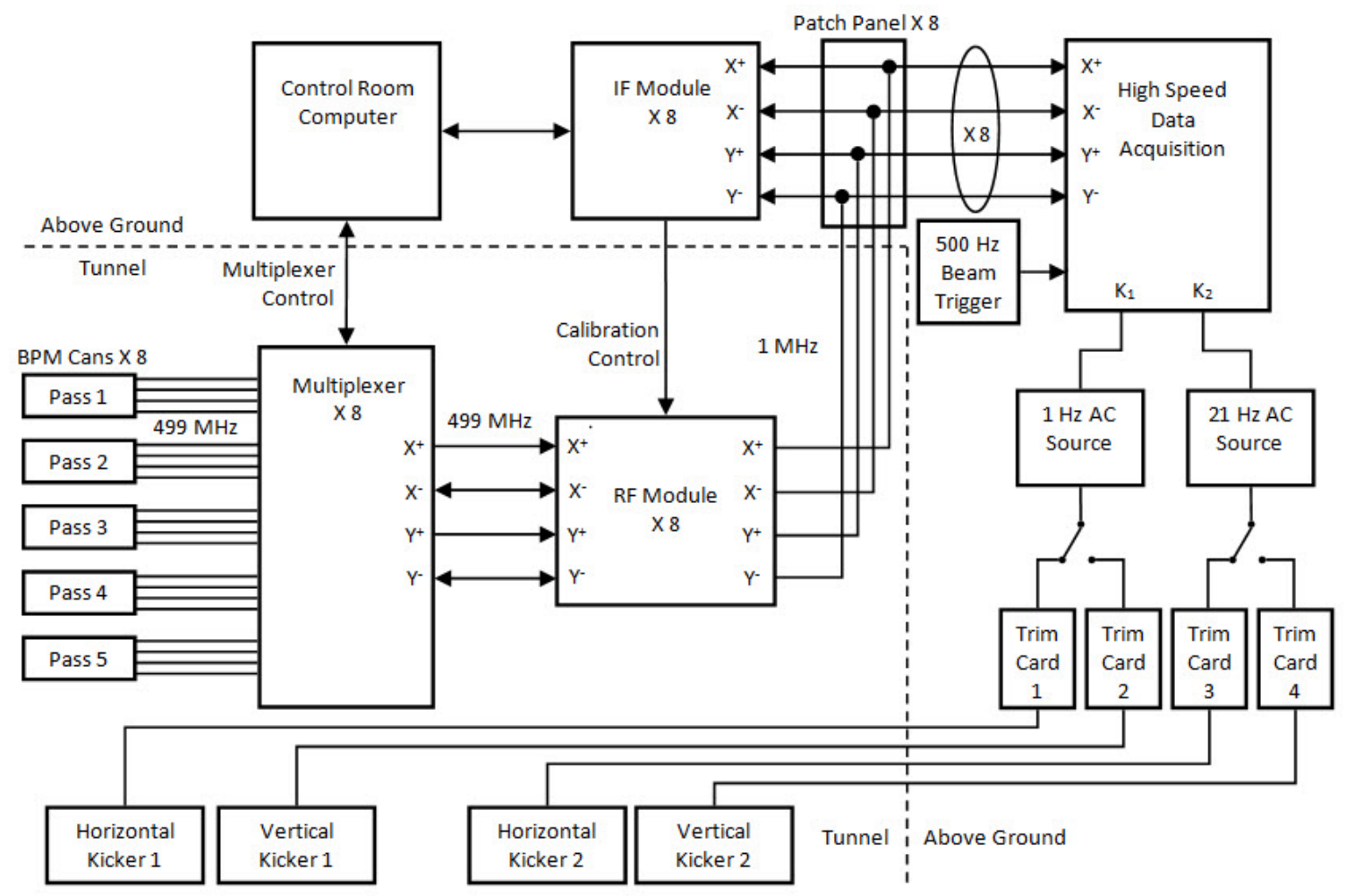

FIG. 16. A schematic representation of the experiment.

\subsection{AIR-CORE KICKER MAGNETS}

Transverse modulation of the beam orbit for this experiment was provided by eight separate individually controlled kicker magnets. The four magnets in the Arc 1 beamline were originally installed in 1998 as part of a machine optics characterization scheme called the $30 \mathrm{~Hz}$ System. A photograph of a pair of these 8" long magnets designated as MAZ1E01H and MAZ1E01V is shown at the top of Fig. 17. The pairs of coils for each magnet are mounted to the 3 " diameter beam tube with plastic tie wraps.

The four magnets for the BSY Recombiner study were specifically designed, built and tested for this research. A photograph of a pair of these 14" long magnets designated as MAK6T04H and MAK6T04V is shown at the bottom of Fig. 17. The pairs of coils for each magnet are mounted in an aluminum holder with a stainless steel band clamp to secure them to the 3" beam tube. The precision machining of the holder allows for better control of the relative coil alignment and overall orientation 

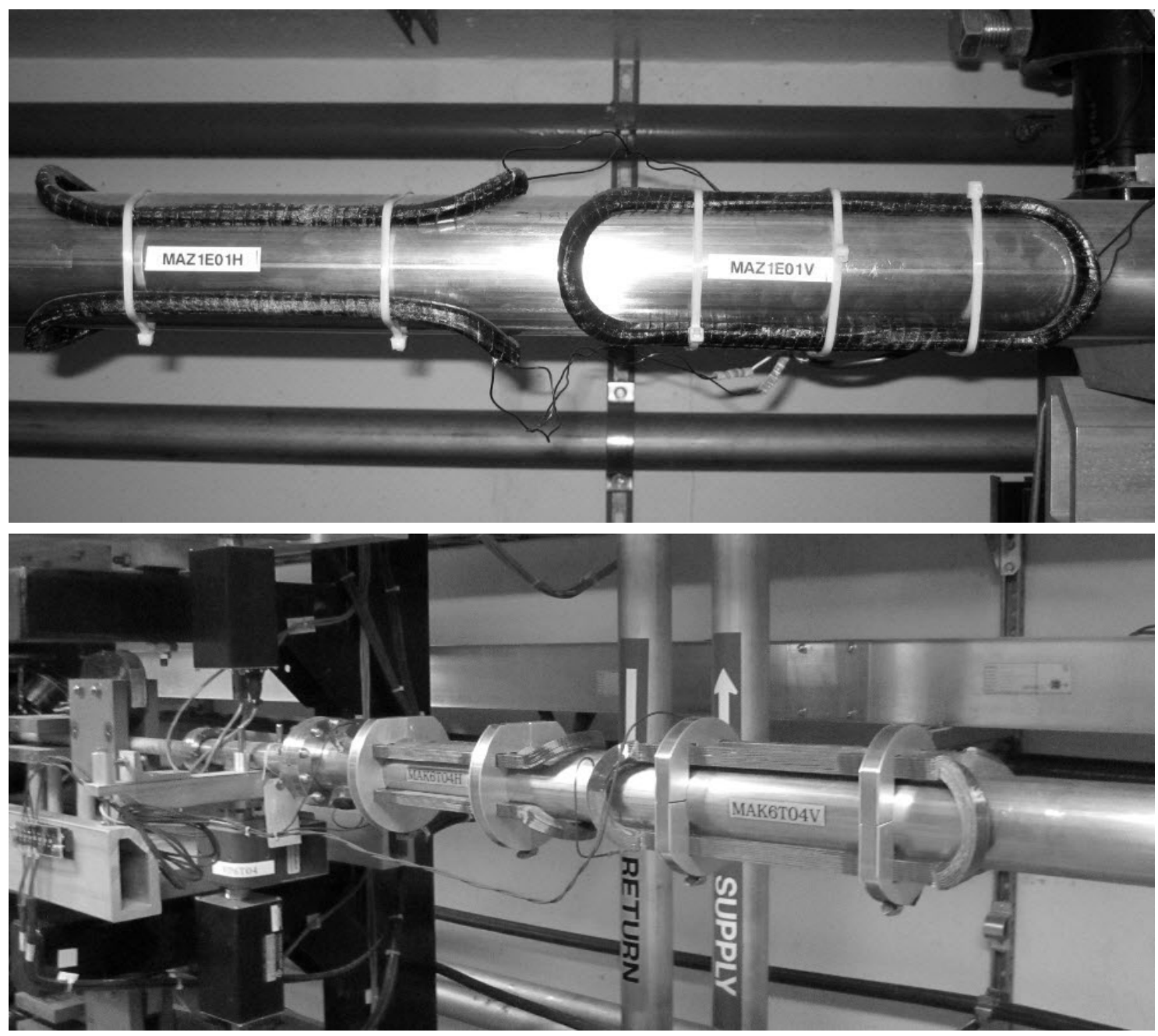

FIG. 17. A picture showing two of the 8" long 60-turn kicker magnets in Arc 1 at the top and two of the 14" long 150-turn kicker magnets in the Arc 6 Recombiner at the bottom.

and position of the kicker assembly.

The selection of a design for the MAK kicker magnets was guided by the requirements for the experiment and by any physical constraints of the system. To adequately explore the fields of the lattice with this technique one needs to modulate the beam across a significant portion of the physical aperture of the beamline. As was shown in section 3.1 the angle for a dipole magnet is given by

$$
\Delta \theta(\mathrm{rad})=2.9979 \times 10^{-4} \frac{B_{0}(\mathrm{G}) L(\mathrm{~cm})}{p_{z}(\mathrm{MeV} / \mathrm{c})} .
$$

So for a given momentum $p_{z}$ we need to find the right combination of central field 


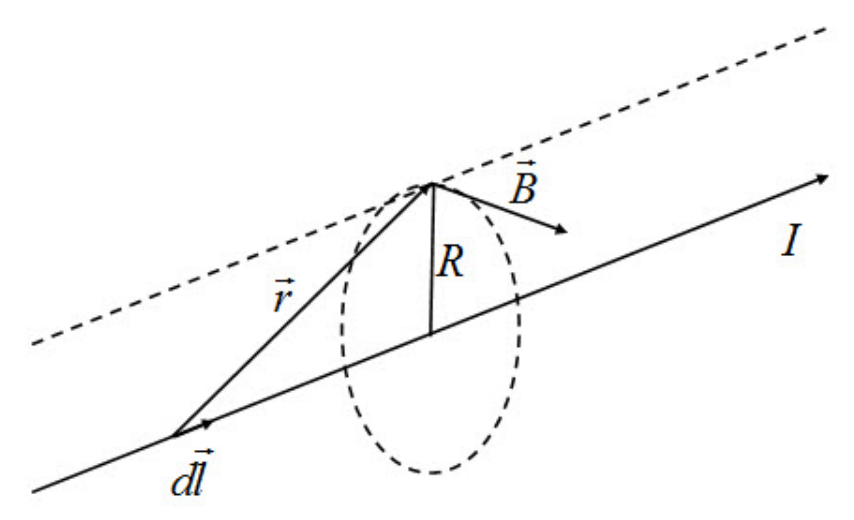

FIG. 18. The coordinate system for determining the magnetic field a distance $R$ from a long straight wire carrying a current $I$.

$B_{0}$ and effective length $L$. In section 3.1 we found that the central field of a dipole scales according to $B_{0}=\mu_{0} n I / h$. The gap $h$ is constrained by the physical size of the beam tube. The number of turns is constrained by the need to keep the current density low enough to prevent the magnets from overheating from ohmic losses. The effective length is limited by the amount of space on the beamline but also by the need to keep the coils as straight as possible along the beam axis. The optimum design needs to strike a balance among all of these competing constraints.

A good estimate of the central field for a kicker magnet can be made using the Law of Biot-Savart and the assumption that the total current for the coil bundle is concentrated at the center. The coordinate system used for what follows is shown in Fig. 18. The integral form of the Biot-Savart relation is given by

$$
\vec{B}=\frac{\mu_{0} I}{4 \pi} \int \frac{d \vec{l} \times \vec{r}}{r^{2}}
$$

For an infinitely long wire we have the familiar result

$$
B_{0}=\frac{\mu_{0} I_{t}}{2 \pi R}
$$

A cross-sectional view of the kicker assembly is shown in Fig. 19. The distance $R$ from the center of the coil to the origin is $4.85 \mathrm{~cm}$ and $I_{t}=150 \mathrm{amp}$-turns for the MAK kicker design. Putting this into the equation for $B_{0}$ provides an estimate of the central field. Numerically we have

$$
B_{0}=\frac{4 \pi \times 10^{-7}(\mathrm{Tm} / \mathrm{A}) \cdot 150}{2 \pi \cdot 4.85 \times 10^{-2}(\mathrm{~m})}=6.185 \times 10^{-4} \mathrm{~T} / \mathrm{A}=6.185 \mathrm{G} / \mathrm{A} .
$$




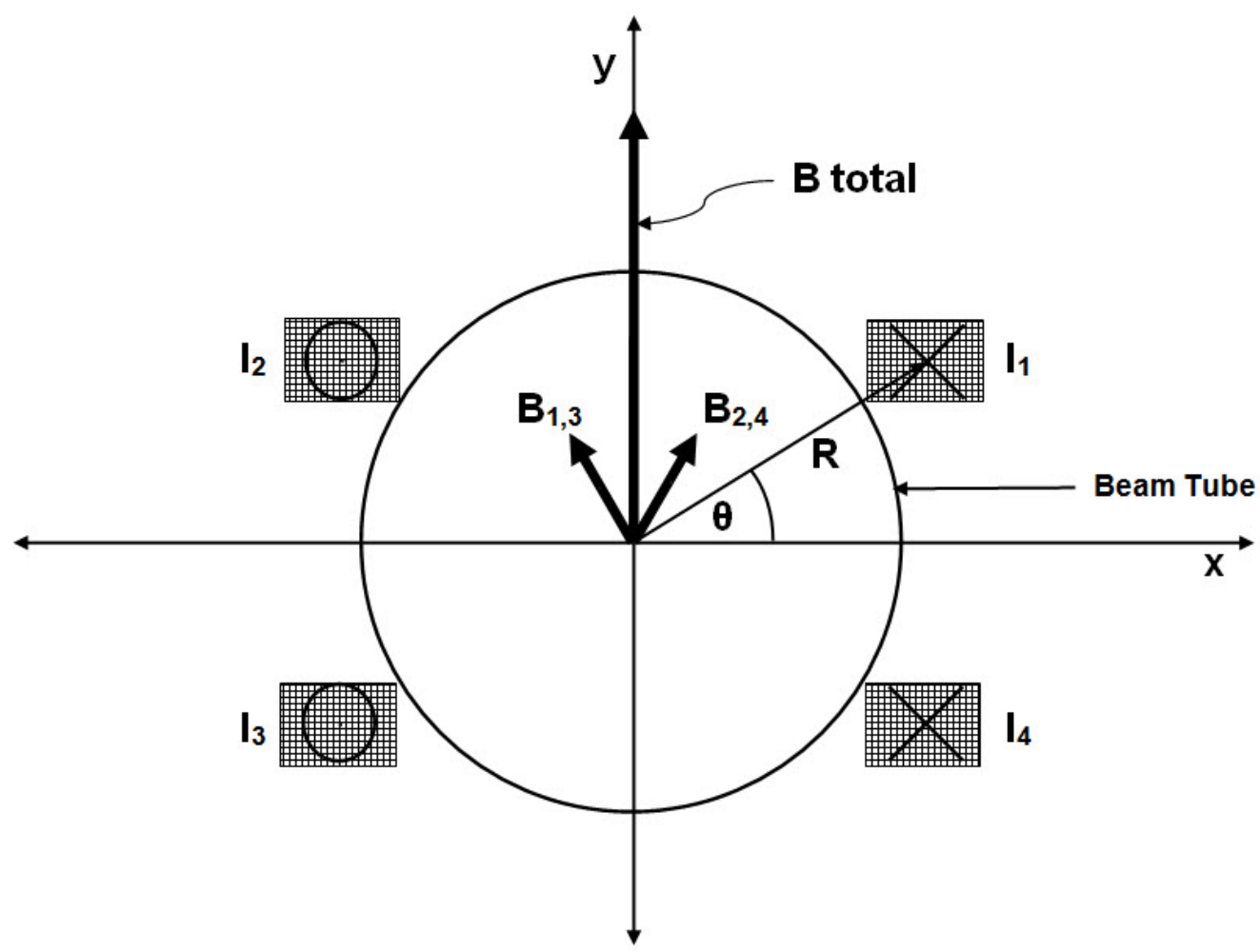

FIG. 19. A cross-sectional view of an MAK magnet showing the field vectors for each of the four current sources and the resultant vertical field.

The field vector for each of the four coil assemblies is shown in Fig. 19. Symmetry guarantees that the field in the $x$-direction is zero. The angle $\theta$ is nominally $30^{\circ}$ giving a total central field along the vertical axis of

$$
B_{t}=4 \cdot B_{0} \cos (\theta)=4 \cdot 6.185 \mathrm{G} / \mathrm{A} \cdot \cos \left(30^{\circ}\right)=21.08 \mathrm{G} / \mathrm{A} \text {. }
$$

The nominal length for the coils is $14 "$ or $35.56 \mathrm{~cm}$. This gives an estimate of the integral field strength of $21.08 \mathrm{G} / \mathrm{A} \times 35.56 \mathrm{~cm}=749.60 \mathrm{G}-\mathrm{cm} / \mathrm{A}$.

The coils are made from 17 AWG kapton coated magnet wire using the winding fixture shown in Fig. 20. The wire is wound under tension into 15 layers of 10 carefully stacked turns for 150 total turns. The ends of the coil assemblies are bent up at an angle of $30^{\circ}$ to allow them to fit around the beam tube and to minimize multipole errors at the ends of the magnets. The coils are then dipped in a high temperature epoxy called HYSOL ${ }^{\circledR}$ and baked in an oven for three hours. A drawing of the coil assemblies is shown in Fig. 21. 


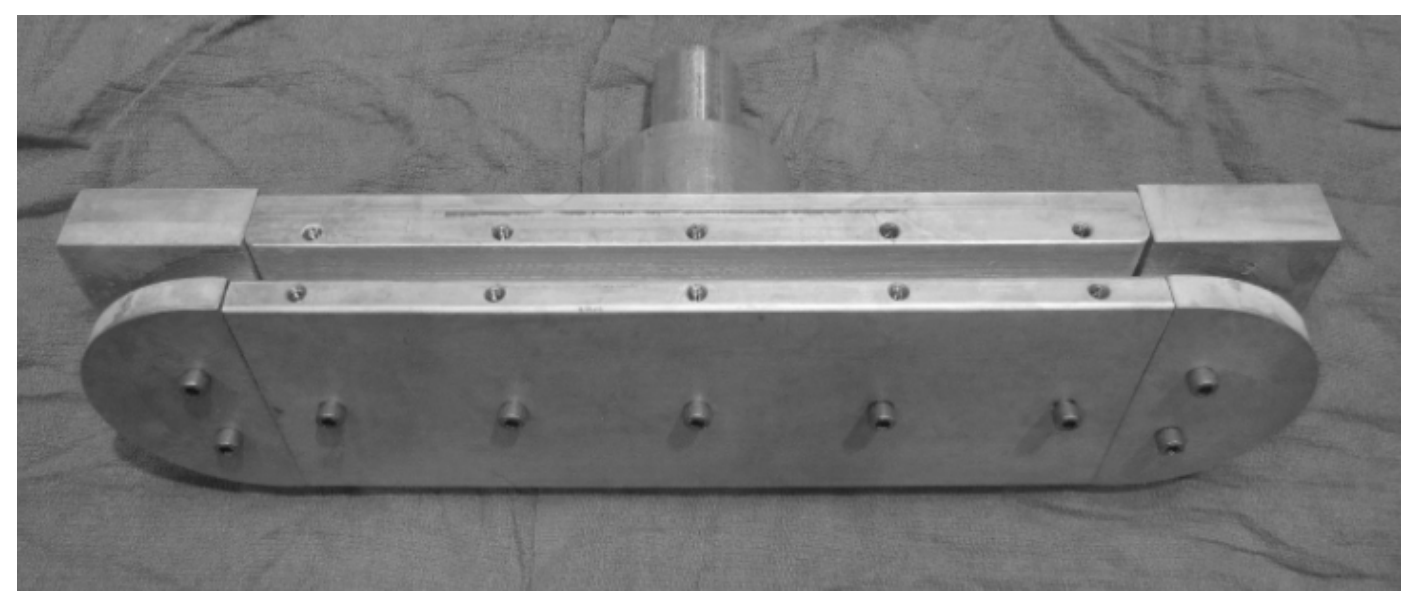

FIG. 20. A picture of the winding fixture that was used to make the coils.
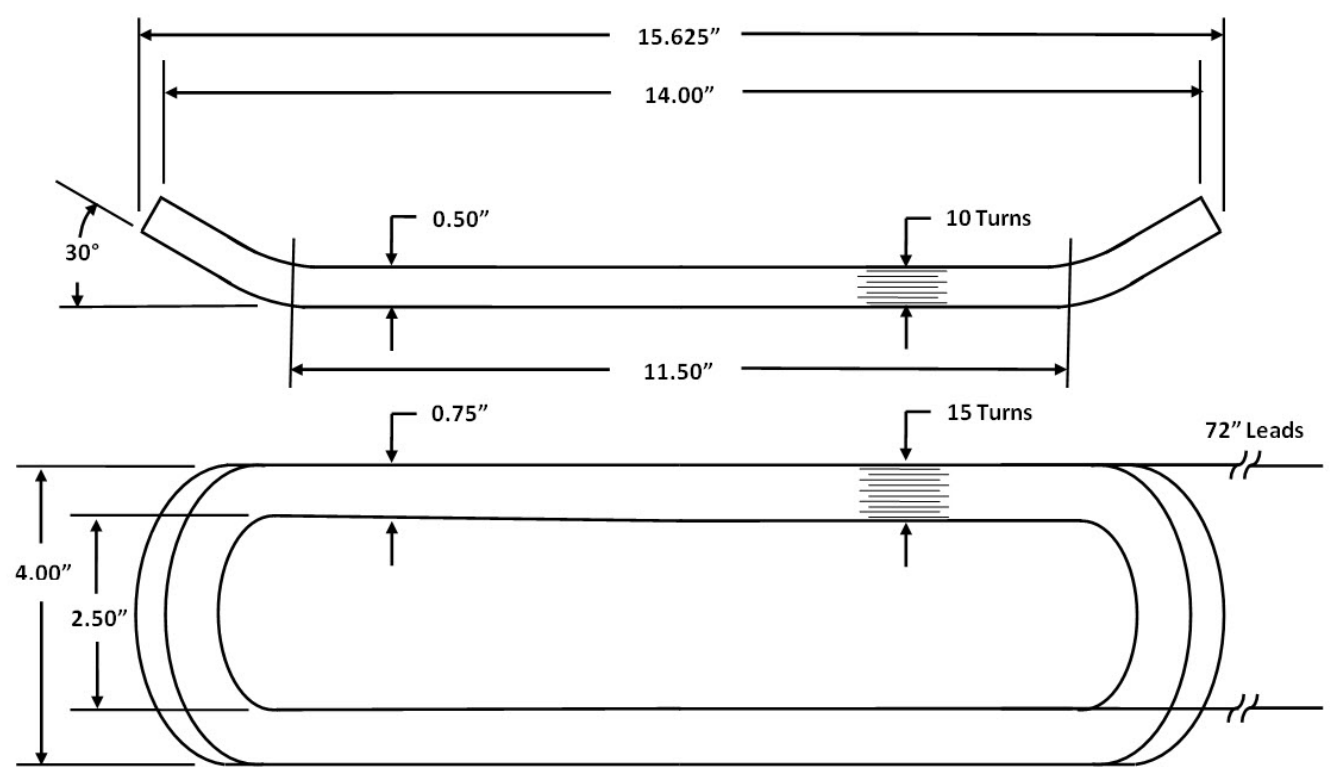

FIG. 21. A drawing of the coil design for the 150 turn MAK kicker magnets that were used in Arc 6. An elevation view (top) and plan view (bottom) is presented. 


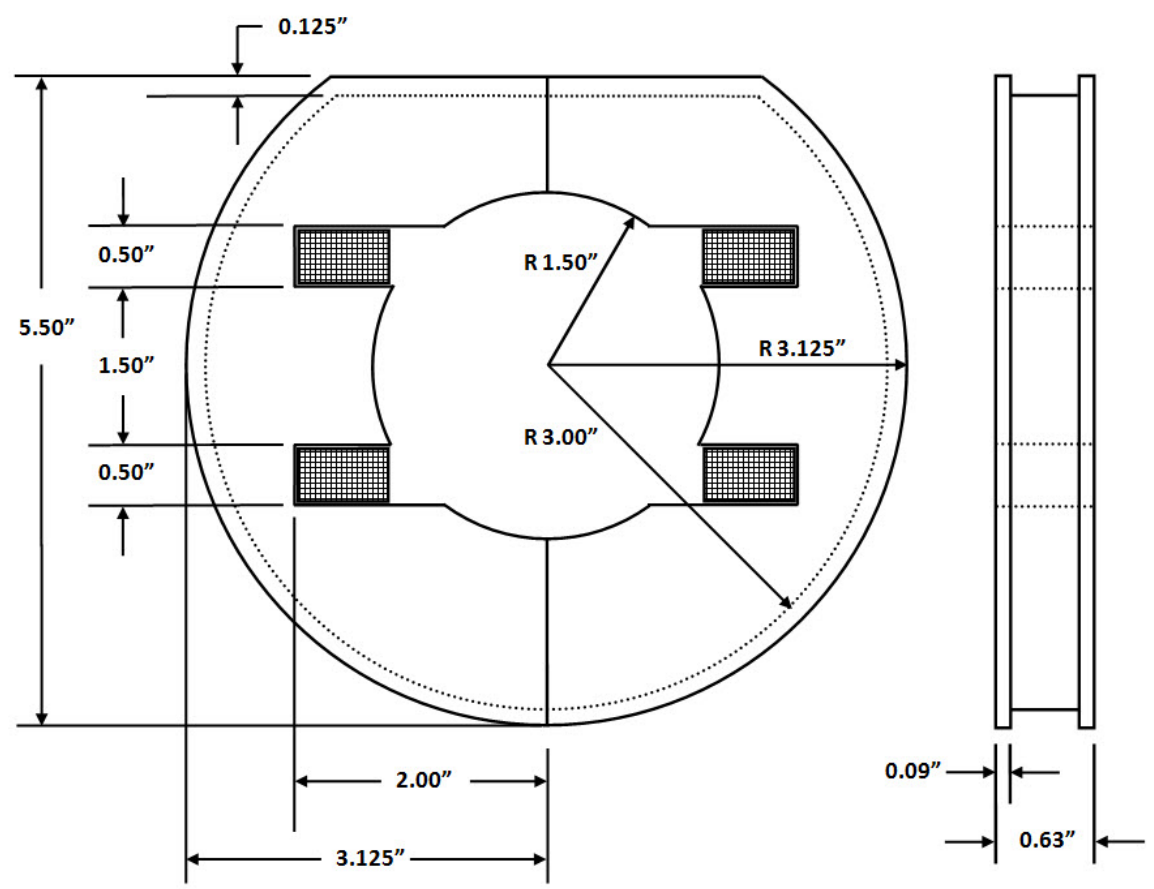

FIG. 22. A drawing of the aluminium clamp for mounting the correctors on the 3" beam tube. At left is a downstream view and shows the coils in the mount. At right is an edge view to show the channel for the stainless steel band clamp.

An aluminum clamp was designed to hold the pair of coils in the proper orientation and to mount them around the 3" diameter beam tube. Two clamps were used for each coil pair and were secured using stainless steel band clamps. A flat section was machined on the round clamp for leveling the completed assembly on the beam tube. A drawing of the clamp is shown in Fig. 22.

The magnet wire has a temperature limit of $200^{\circ} \mathrm{C}$. A test of a completed magnet assembly was performed in the lab to verify that it did not exceed this temperature rating. The test was done with $5 \mathrm{~A}$ of DC current for over four hours to allow the temperature to come to equilibrium. The peak temperature was around $80^{\circ} \mathrm{C}$ which is well below the limit. The results are shown in Fig. 23.

Both the MAZ and MAK kicker magnets were measured for field quality before they were installed on the beamline. The integrated dipole strength along the midplane was measured at several DC currents to develop a map of magnetic field versus excitation current for use in the control of the magnets. At each current the field is measured with a Hall Probe starting well outside the magnet where only the earth's 


\section{Coil Surface Temperature}

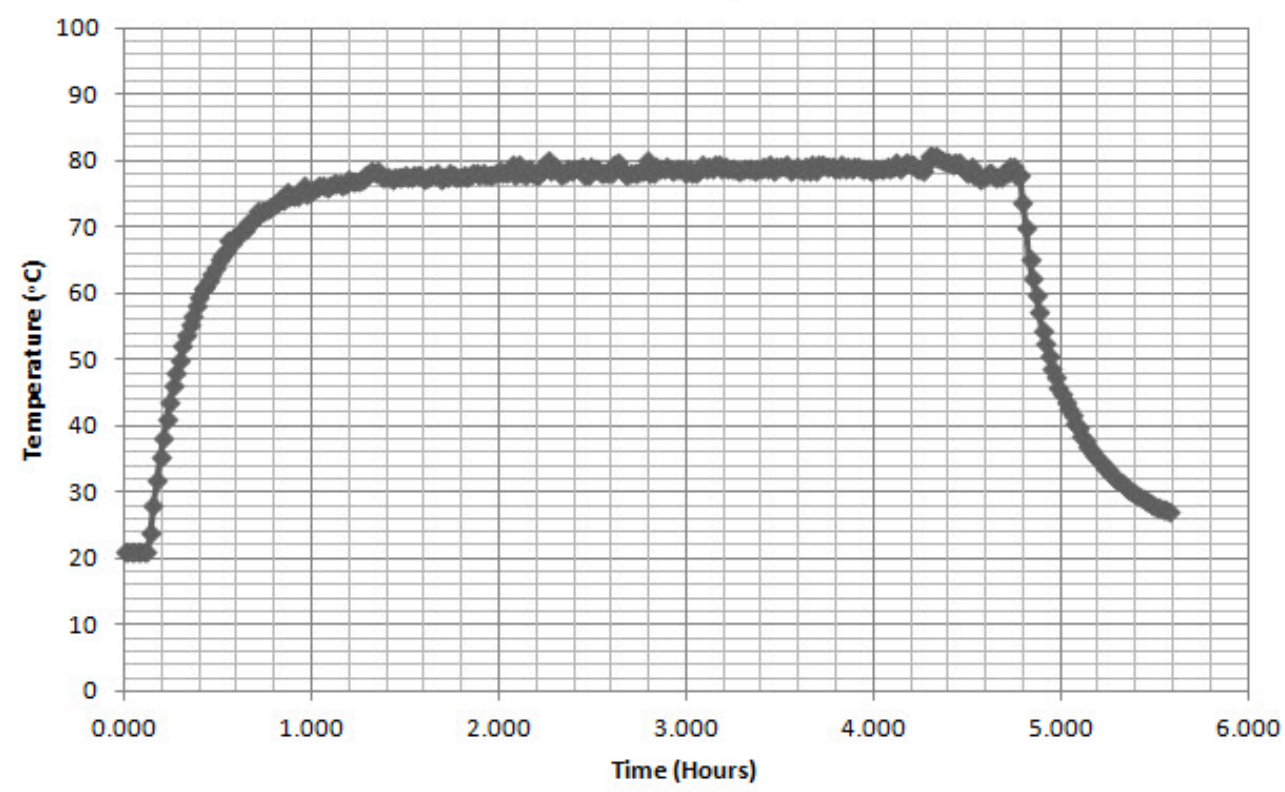

FIG. 23. A graph of temperature data for a coil pair operating at 5 Amps DC current.

field is present. The probe is stepped along a straight line through the magnet and out the other side in $0.5 \mathrm{~cm}$ increments until once again only the earth's field is evident. The field measurements at each point are added and then multiplied with the total length of the path through the magnet. The data for the MAZ magnet is from the CEBAF database and includes a single track along the longitudinal $z$ axis at $x=y=0$. The data for the MAK magnets was also taken using a Hall probe but this time along thirteen different tracks in the $y=0$ midplane from $x=-3 \mathrm{~cm}$ to $x=+3 \mathrm{~cm}$ in $0.5 \mathrm{~cm}$ increments. The field map along $x=y=0$ is shown for each magnet type in Fig. 24. The earlier estimate of the integrated field strength of the MAK magnet was $749.60 \mathrm{G}-\mathrm{cm} / \mathrm{A}$. The value from the lab measurement was 745.42 G-cm/A which is within $2 \%$ of the estimate. All of the magnet measurement data is corrected for the earth's field which is on order $0.5 \mathrm{G}$. The generalized coordinate system for resolving the field into components is shown in Fig. 25. The data [17] for the earth's field at the location of the Magnet Measurement Facility is shown in Table 5 .

The thirteen individual tracks of Hall probe data for the MAK magnet is shown in Fig. 26. The plots show the quality of the left-right symmetry of the field. The 

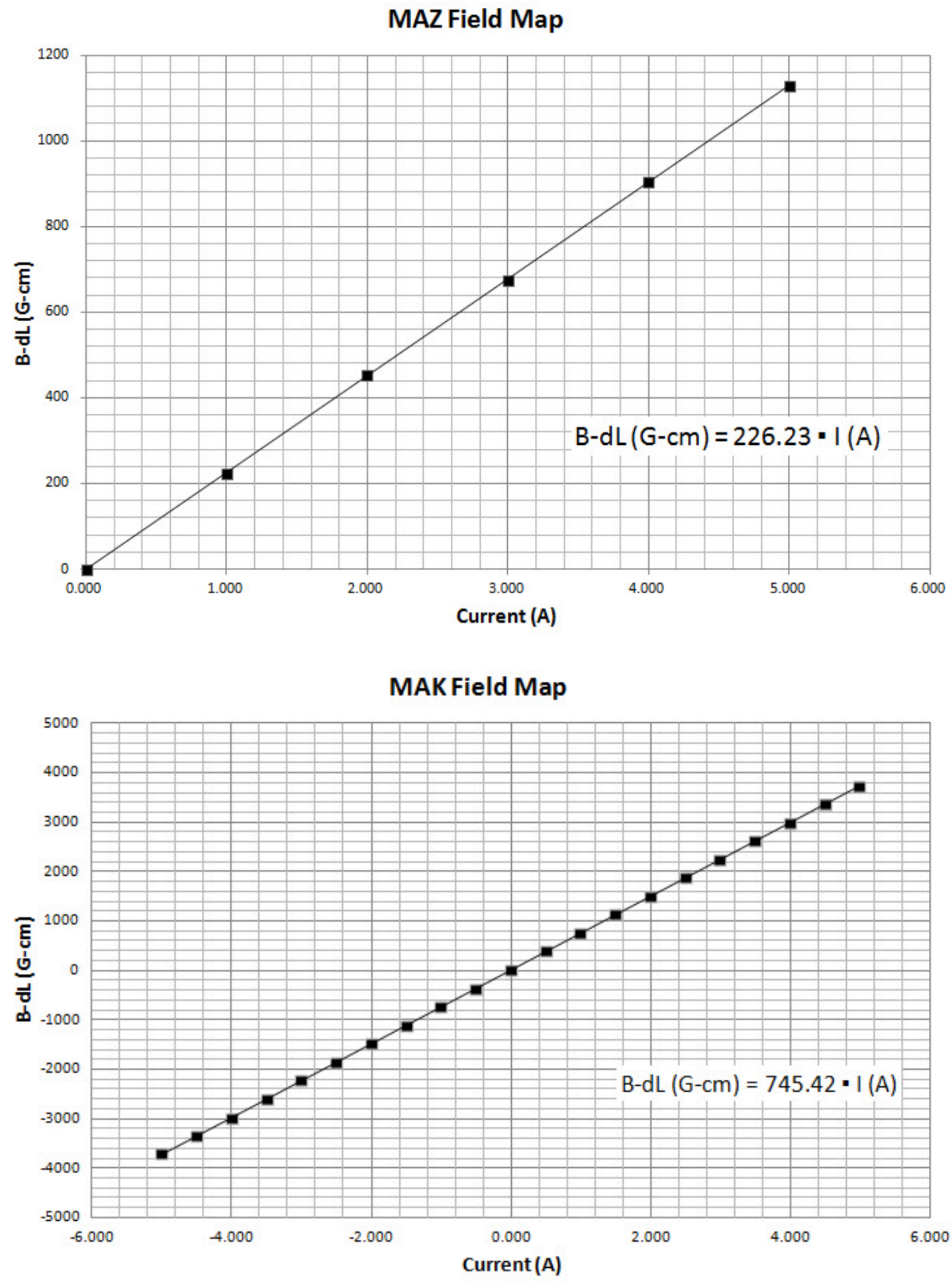

FIG. 24. Field map graphs for the MAZ magnet at the top and the MAK magnet at the bottom. Both data are integrated along $z$ at $x=y=0$. 


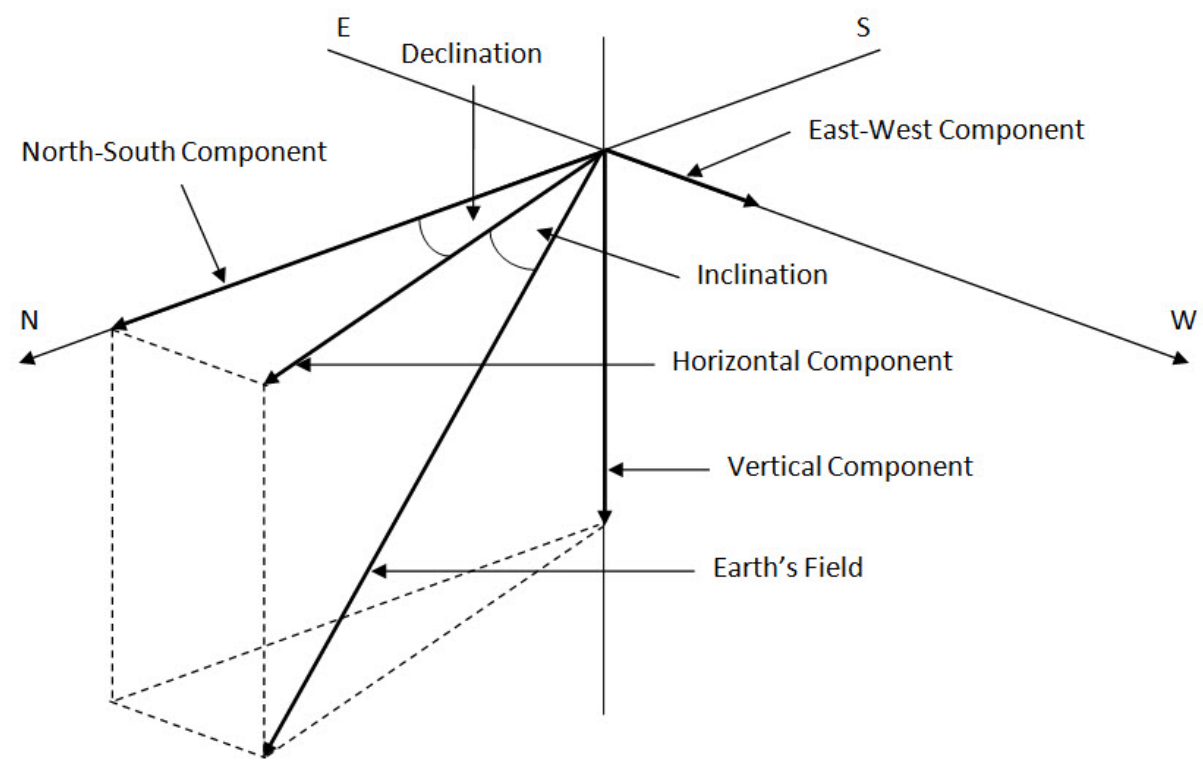

FIG. 25. The generalized coordinate system for describing the earth's field.

TABLE 5. The Earth's magnetic field at Jefferson Lab.

\begin{tabular}{lcc}
\hline \hline Parameter & Value & Unit \\
\hline Declination & -10.71402 & $\mathrm{deg}$ \\
Inclination & 64.63293 & $\mathrm{deg}$ \\
North-South Component & 214.0066 & $\mathrm{mG}$ \\
East-West Component & -404.912 & $\mathrm{mG}$ \\
Horizontal Component & 217.7954 & $\mathrm{mG}$ \\
Vertical Component & 459.3534 & $\mathrm{mG}$ \\
Earth's Field & 508.3738 & $\mathrm{mG}$ \\
\hline \hline
\end{tabular}




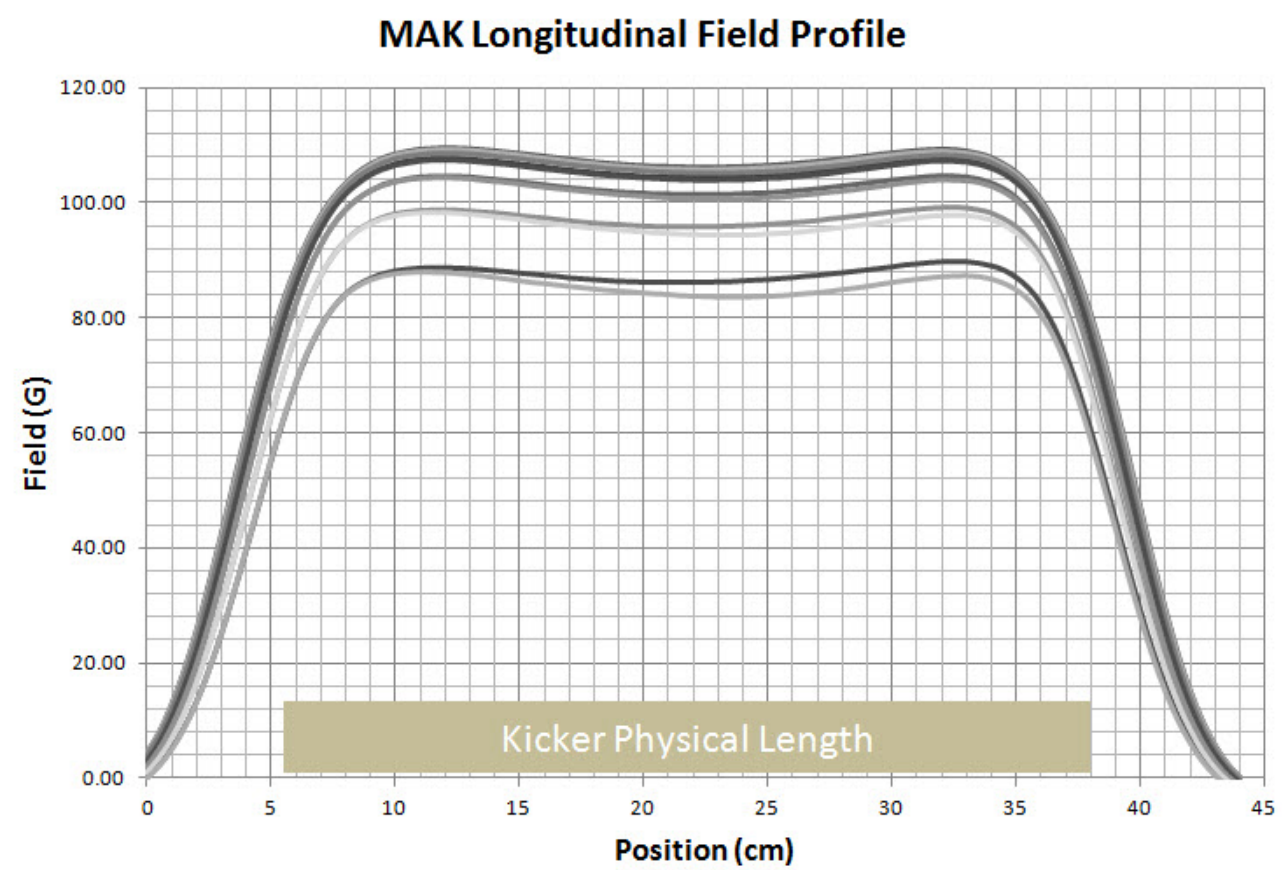

FIG. 26. A graph of the magnetic field versus the longitudinal position for the MAK magnet showing all thirteen tracks. The box represents the length of the magnet.

actual corrector length is represented by the rectangle to show how the edge effects are related to the real magnet. Figure 27 shows the integrated field strength versus the transverse position in the midplane of the magnet. The data are normalized to the central trajectory and show reasonable flatness out to about $1 \mathrm{~cm}$.

In this experiment the beam modulation of the first kicker magnet can result in a large transverse position offset in the second kicker magnet which is simultaneously modulating. Its important to try and minimize the multipole content of the kickers themselves so that their nonlinearity doesn't significantly add to that of the lattice being investigated. Bench measurements of the harmonic content of both the MAZ and MAK magnets were conducted using a rotating coil system.

A rectangular loop of wire is printed onto a circuit board, mounted onto a rotational mechanism and then spun inside the magnet at a low frequency. The outer edge of the coil is located a fixed distance from the axis of rotation which is called the reference radius. The time rate of change of the magnetic flux induces a voltage in the loop which is then recorded. Fourier decomposition of the signal is used to analyze the data and determine the strength of each harmonic relative to the $n=1$ 


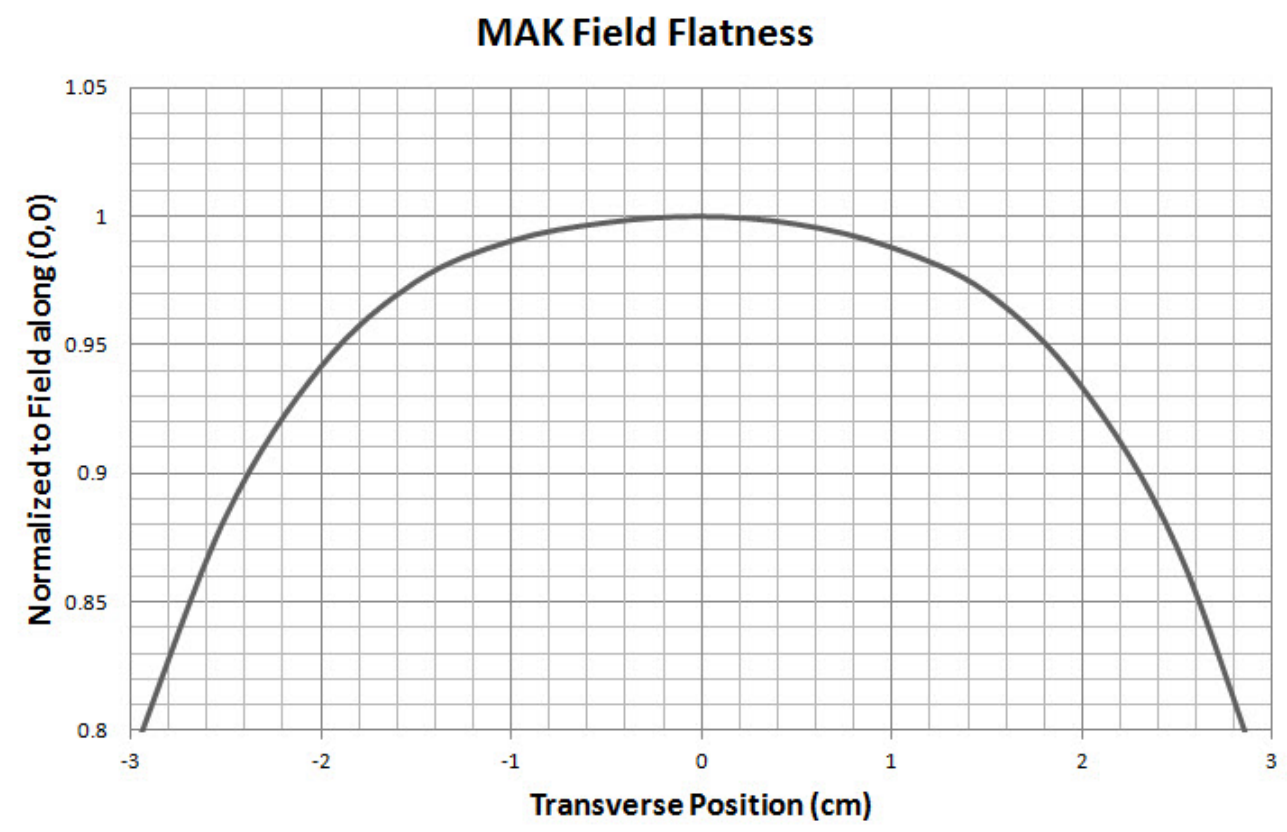

FIG. 27. A graph of the integrated field strength versus the transverse position in the midplane. The data is normalized to the track at $x=y=0$.

dipole term.

The voltage generated in a rotating loop of wire at radius $r$ with the return on the axis of rotation is given by

$$
V=-\frac{d \Phi}{d t}
$$

where $\Phi$ is the total flux in the loop. The total flux can also be written as

$$
\Phi=L_{e f f} \int B(s) d s .
$$

For each harmonic number the induced voltage is related to the field strength by

$$
\int V^{(n)} d t=-L_{e f f} \int B^{(n)} d s .
$$

The calculated results for the $n$th harmonic at the reference radius can be scaled to any radius using

$$
R_{\text {new }}^{n}=R_{\text {ref }}^{n}\left[\frac{r_{\text {new }}}{r_{\text {ref }}}\right]^{n-1}
$$

The results for the MAZ and MAK magnets at a radius of $1 \mathrm{~cm}$ and $0.5 \mathrm{~cm}$ is shown in Figs. 28 and 29 respectively. Beyond $n=9$ the harmonic strength was 
MAZ Harmonics at $1.0 \mathrm{~cm}$

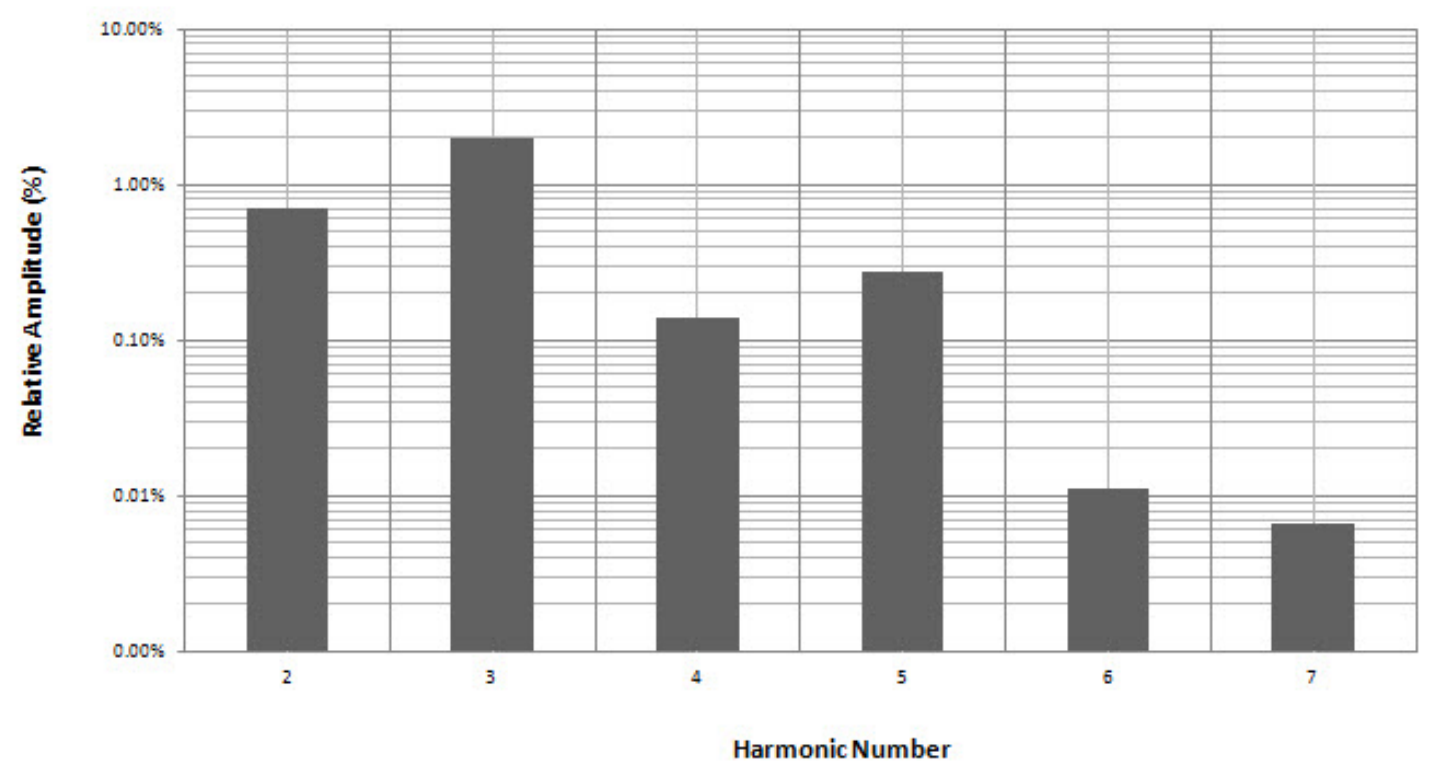

MAZ Harmonics at $0.5 \mathrm{~cm}$

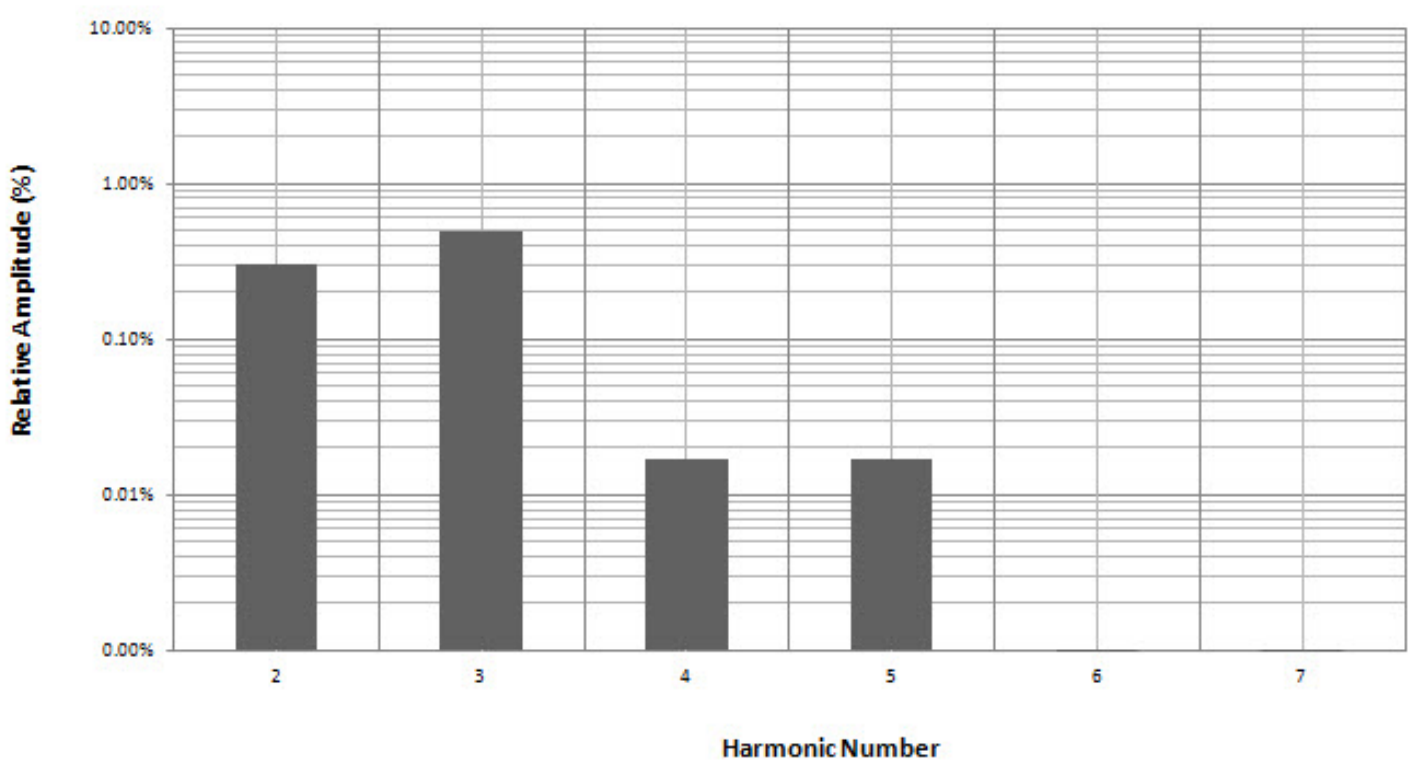

FIG. 28. Harmonic content of MAZ kicker for $1.0 \mathrm{~cm}$ radius at the top and $0.5 \mathrm{~cm}$ radius at the bottom. 
MAK Harmonics at $1.0 \mathrm{~cm}$

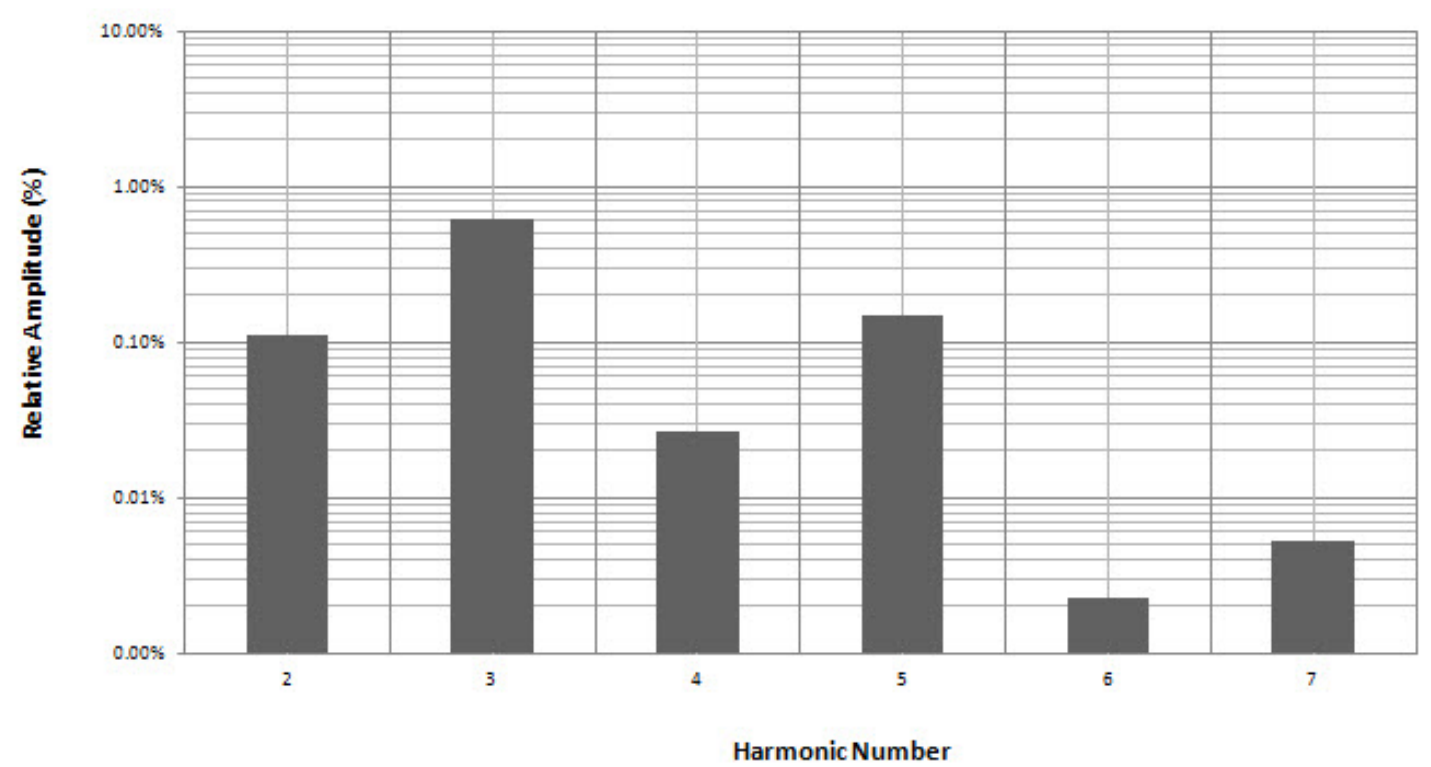

MAK Harmonics at $0.5 \mathrm{~cm}$

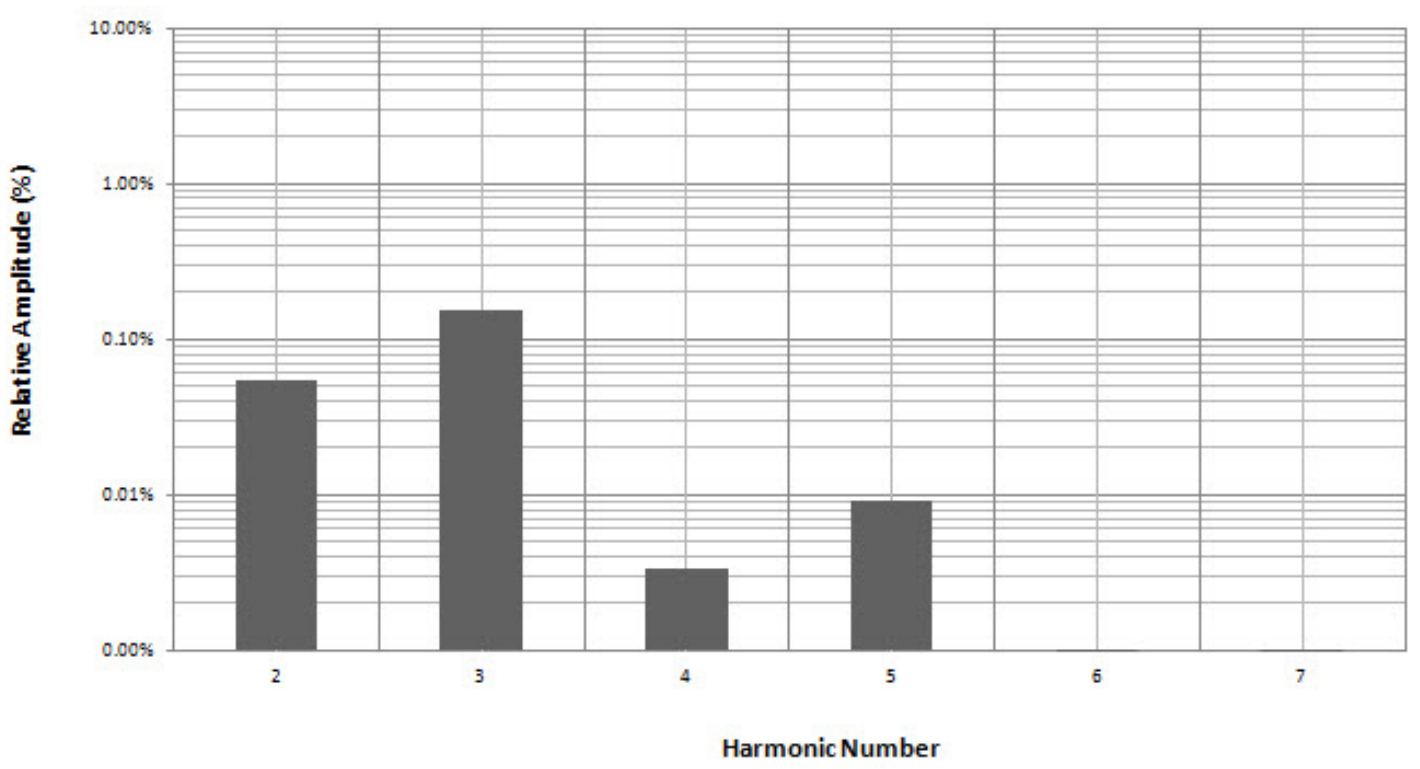

FIG. 29. Harmonic content of MAK kicker for $1.0 \mathrm{~cm}$ radius at the top and $0.5 \mathrm{~cm}$ radius at the bottom. 


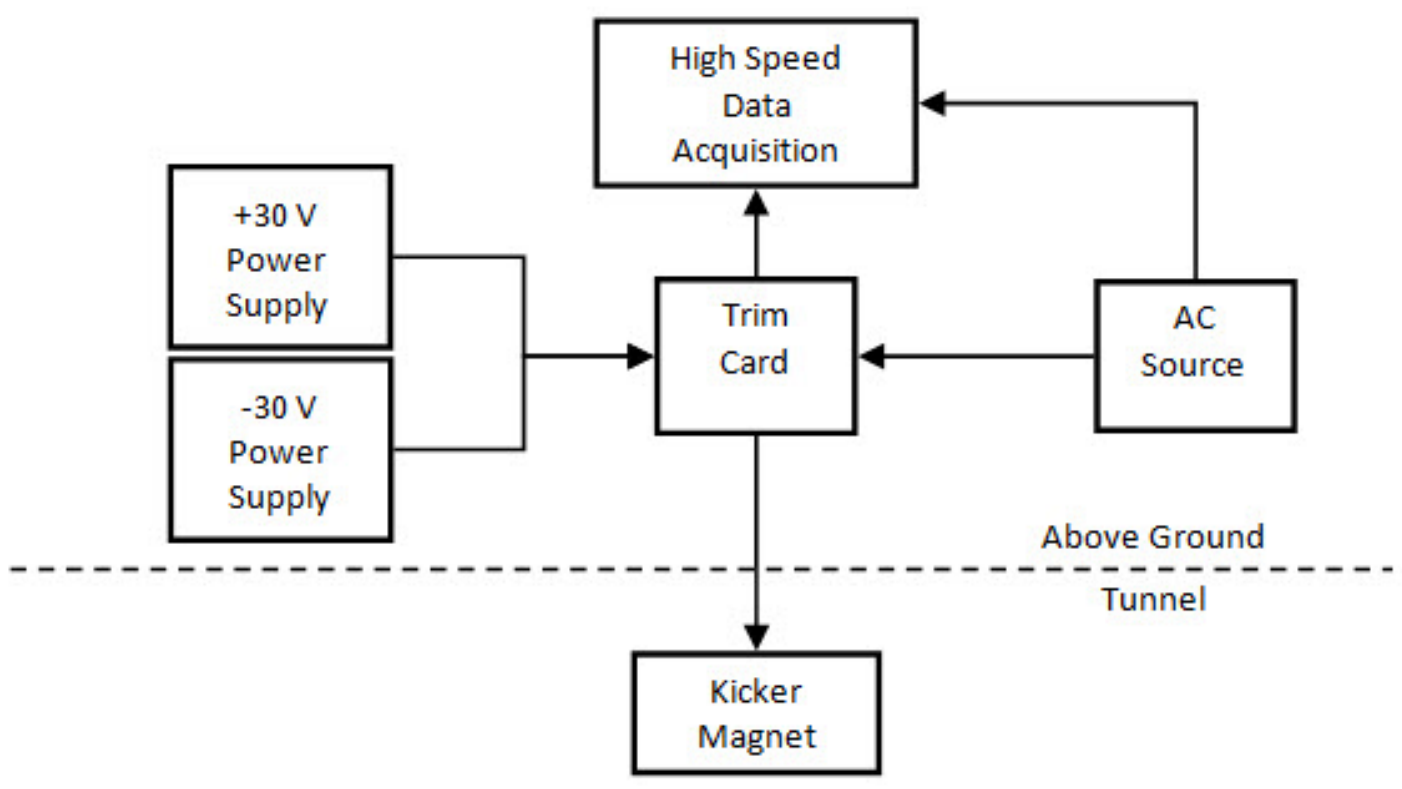

FIG. 30. A schematic of the trim magnet system.

vanishingly small for both magnet types. The MAK magnet has better results overall due to the uniformity and precision mounting of the coils.

The hundreds of quadrupole, sextupole, and orbit correction magnets around the accelerator are controlled with what is referred to as the Trim Magnet System. A schematic representation is shown in Fig. 30. Each equipment rack in the service building contains thirty-two trim cards that are independently controlled across a serial network from the control room. The digital to analog converter (DAC) is housed within an isothermal regulator module to provide precision control of the magnet current. The DAC is connected to a shunting preamplifier to control the current. The racks are equipped with a positive and negative polarity power supply operating at $30 \mathrm{~V}$ which source the current for the trim cards within that rack.

The cards for this experiment are specially modified to take their setpoint from an external input. The DAC output within the regulator module was disconnected from the control circuit and replaced by the external input. An HP function generator was then connected to the input through a patch panel to control the current set point for each of the kicker magnets. The signal sent to the trim card was also sent to the data acquisition system to be synchronously sampled with the BPM data. The 
output from the shunting preamplifier, which scales with the current returning from the magnet was also sent to the data acquisition system.

\subsection{BEAM POSITION MONITORS}

The CEBAF accelerator is a highly instrumented machine with over 600 Beam Position Monitors distributed throughout the $6.7 \mathrm{~km}$ of beamline. In this thesis two distinct types of $1 / 4$ wave antenna-style BPMs $[18,19]$ are used, which are designated M20 and M15. A drawing for each type of BPM can is shown in Fig. 31. The larger M20 is used in Arc 1, Arc 2, and the Extraction region of the accelerator with the smaller M15 can used elsewhere.

The $1497 \mathrm{MHz}$ micropulse structure of the electron beam creates an electric field which couples to the $1 / 4$ wave antennas within the BPM can. An electrical schematic is shown in Fig. 32 for illustration. The beam position is proportional to the difference divided by the sum of the induced voltages on the antennae which is given by

$$
r \propto \frac{V^{+}-V^{-}}{V^{+}+V^{-}}=\frac{\Delta}{\Sigma}
$$

For a perfectly centered beam the voltage for each channel is the same which corresponds to $r=0$. A beam that is offset from the central trajectory will result in an imbalance between the two signals. While the sum of the signals remains the same we now have a nonzero difference signal which is interpreted as a position offset.

The proportionality constant $k$ depends on the can geometry. For the M15 BPM $k=18.86 \mathrm{~mm}$ and for the M20 BPM $k=25.56 \mathrm{~mm}$. A constant $\alpha$ is introduced to account for any mechanical or electrical mismatch between the two channels. The relation for $r$ is now given by

$$
r=k \frac{V^{+}-\alpha V^{-}}{V^{+}+\alpha V^{-}}
$$

The BPM has two opposing pairs of antennae designated $X^{+}, X^{-}, Y^{+}, Y^{-}$that are oriented $45^{\circ}$ to the lab frame. This is to prevent synchrotron light, emitted from the beam in the horizontal or vertical bend plane, from striking the antennas. To find $\alpha_{y}$ an RF calibration signal is applied to the $X^{-}$antenna in the absence of beam. Taking the ratio of $Y^{+}-Y_{o f f}^{+}$to $Y^{-}-Y_{o f f}^{-}$, where the offset signals are recorded with the beam and calibration source off, provides the value for $\alpha_{Y}$. To find $\alpha_{X}$ the same procedure is used but this time the RF calibration signal is applied to the $Y^{-}$ 

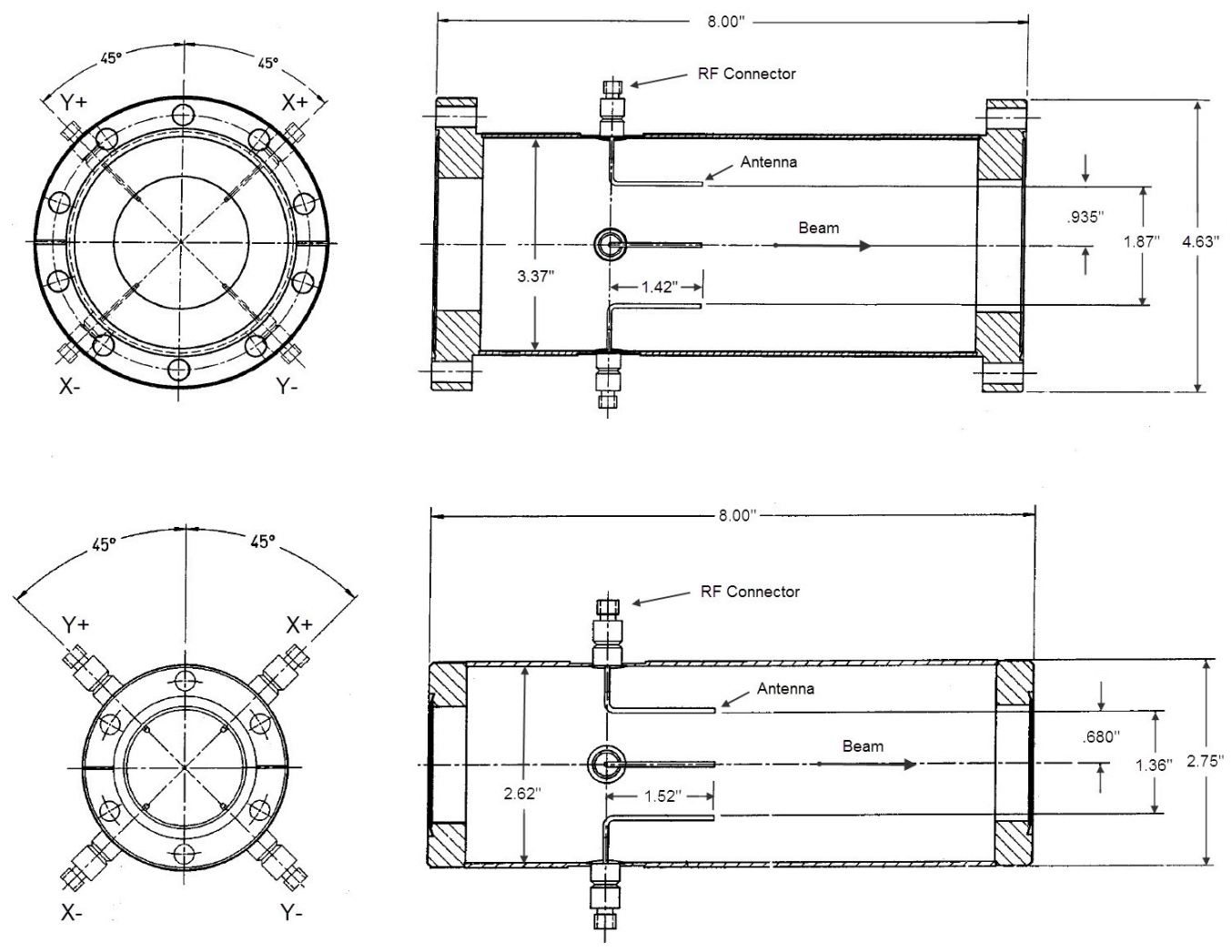

FIG. 31. Schematic drawing of an M20 BPM at the top and an M15 BPM at the bottom.

antenna. The expression for the two constants is given by

$$
\begin{aligned}
\alpha_{x} & =\frac{X^{+}-X_{o f f}^{+}}{X^{-}-X_{o f f}^{-}}, \\
\alpha_{y} & =\frac{Y^{+}-Y_{o f f}^{+}}{Y^{-}-Y_{o f f}^{-}} .
\end{aligned}
$$

An example calibration run is shown in Fig. 33. When the $Y^{-}$calibration signal is on we see that the $X^{+}$and $X^{-}$signals are nearly equal while the $Y^{+}$signal is weakest since its furthest from the calibration source. When the calibration signal is off we see that all four offset signals are just a few hundred counts.

The $X$ and $Y$ position of the beam in the rotated frame is then fully determined and designated $X_{\text {rot }}$ and $Y_{\text {rot }}$ with the two equations written as

$$
X_{r o t}=k \frac{\left(X^{+}-X_{o f f}^{+}\right)-\alpha_{x}\left(X^{-}-X_{o f f}^{-}\right)}{\left(X^{+}-X_{o f f}^{+}\right)+\alpha_{x}\left(X^{-}-X_{o f f}^{-}\right)},
$$




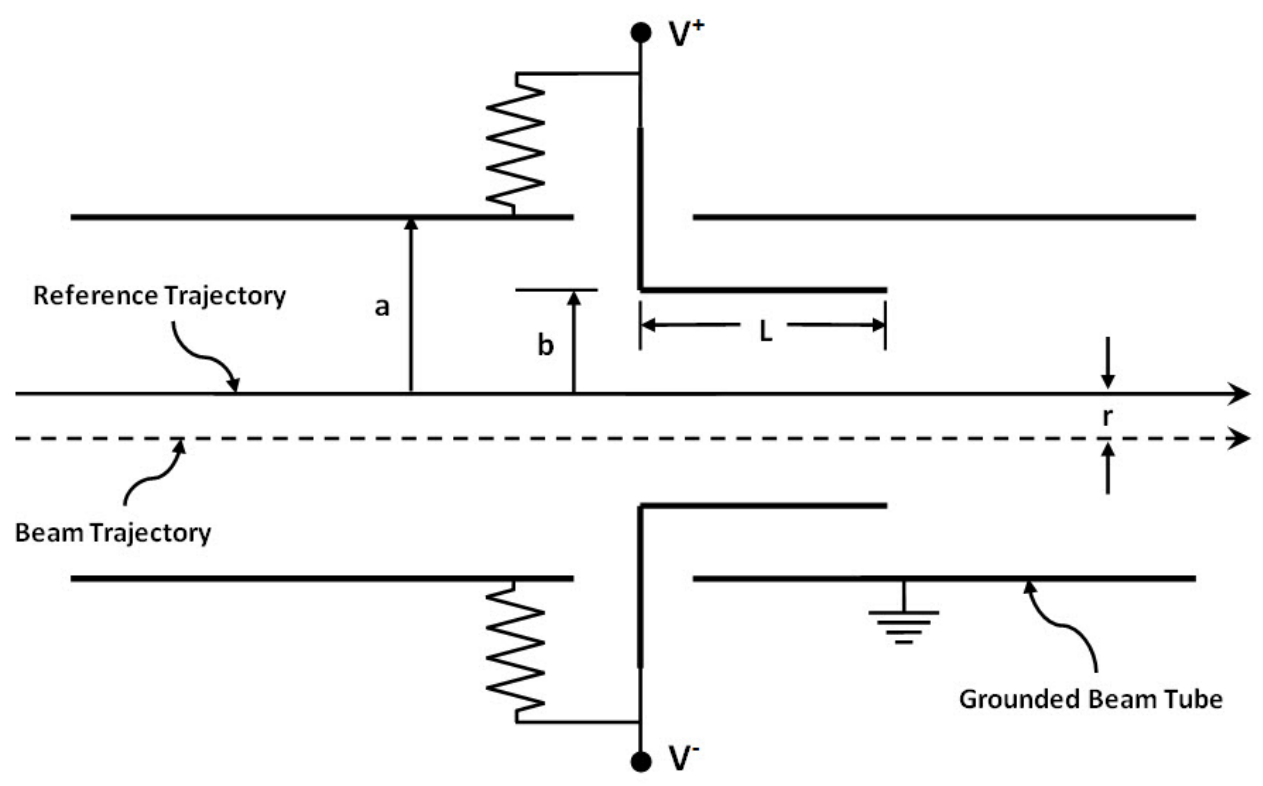

FIG. 32. An electrical schematic for a CEBAF Beam Position Monitor. The electron beam creates a transverse electromagnetic field that couples to the $V^{+}$and $V^{-}$ antennae. The measured position will depend on the difference in antenna voltages.

$$
Y_{\text {rot }}=k \frac{\left(Y^{+}-Y_{o f f}^{+}\right)-\alpha_{y}\left(Y^{-}-Y_{o f f}^{-}\right)}{\left(Y^{+}-Y_{o f f}^{+}\right)+\alpha_{y}\left(Y^{-}-Y_{o f f}^{-}\right)} .
$$

The beam position in the lab frame is then determined by a simple coordinate rotation by $45^{\circ}$ given by

$$
\begin{aligned}
& X=\frac{1}{\sqrt{2}}\left(X_{\text {rot }}-Y_{\text {rot }}\right), \\
& Y=\frac{1}{\sqrt{2}}\left(X_{\text {rot }}+Y_{\text {rot }}\right) .
\end{aligned}
$$

The error in our knowledge of alpha can be determined from the standard rules for error propagation. When adding or subtracting two arbitrary signals $A$ and $B$ the error is given by

$$
\Delta R=\sqrt{(\Delta A)^{2}+(\Delta B)^{2}} .
$$

When multiplying or dividing two arbitrary signals $A$ and $B$ the error is given by

$$
\Delta R=R \sqrt{\left(\frac{\Delta A}{A}\right)^{2}+\left(\frac{\Delta B}{B}\right)^{2}} .
$$




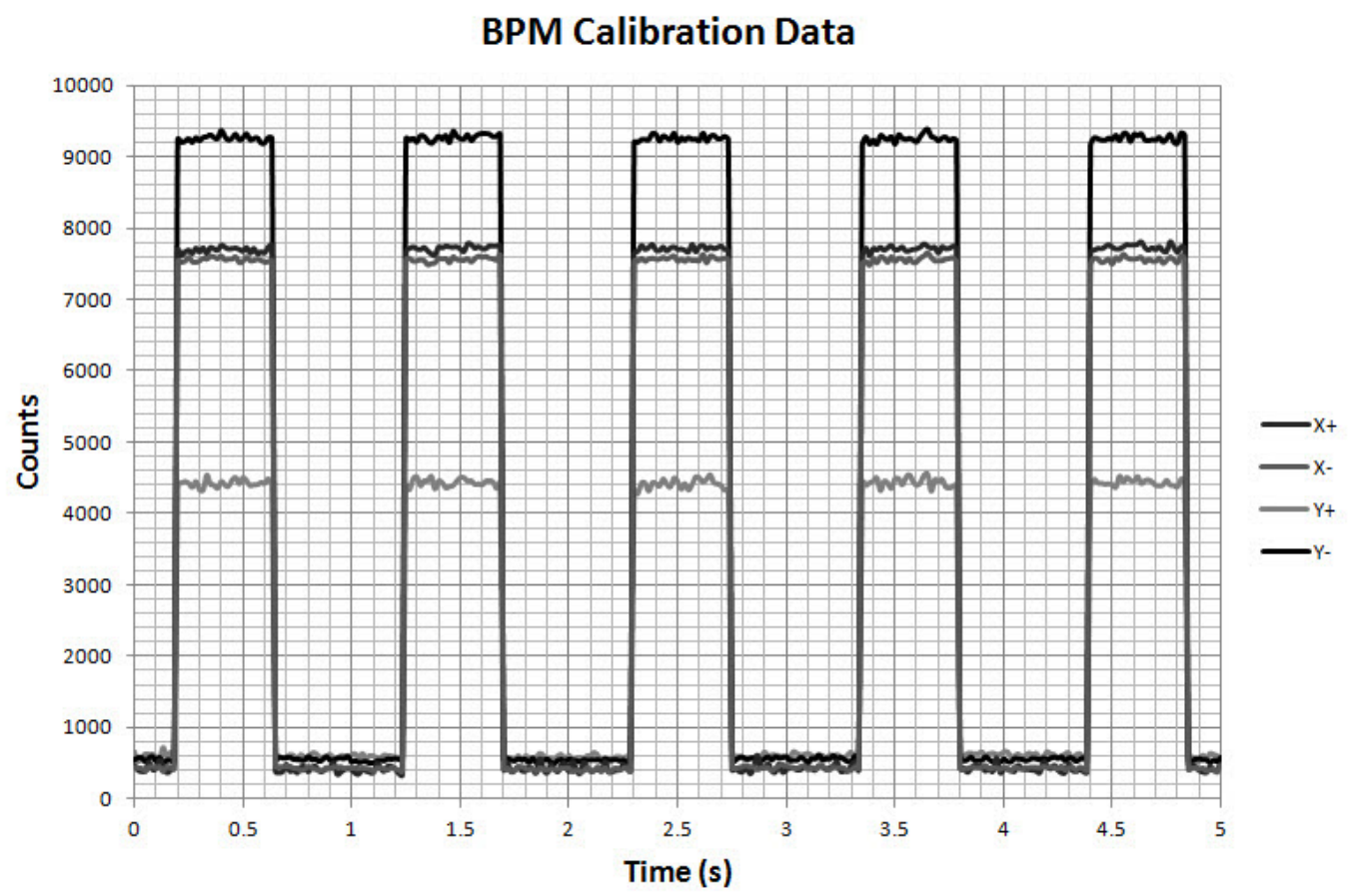

FIG. 33. A plot of four BPM wire signals during the alpha calibration procedure.

Using the RMS value of the wire signals with the calibration oscillators on and with the calibration oscillators off and then applying the above rules we can determine the relative error in $\alpha_{x}$ and $\alpha_{y}$. The error equations are then given by

$$
\begin{aligned}
& \frac{\Delta \alpha_{x}}{\alpha_{x}}=\sqrt{\frac{\left(\Delta X^{+}\right)^{2}+\left(\Delta X_{o f f}^{+}\right)^{2}}{\left(X^{+}-X_{o f f}^{+}\right)^{2}}+\frac{\left(\Delta X^{-}\right)^{2}+\left(\Delta X_{o f f}^{-}\right)^{2}}{\left(X^{-}-X_{o f f}^{-}\right)^{2}}}, \\
& \frac{\Delta \alpha_{y}}{\alpha_{y}}=\sqrt{\frac{\left(\Delta Y^{+}\right)^{2}+\left(\Delta Y_{o f f}^{+}\right)^{2}}{\left(Y^{+}-Y_{o f f}^{+}\right)^{2}}+\frac{\left(\Delta Y^{-}\right)^{2}+\left(\Delta Y_{o f f}^{-}\right)^{2}}{\left(Y^{-}-Y_{o f f}^{-}\right)^{2}}} .
\end{aligned}
$$

For this thesis there were eight BPMs in the Arc 1 beamline and eight BPMs in the Arc 6 Recombiner beamline that were included in the experiment. The calibration data for all sixteen BPMs is captured in the next three tables. The data for calibration oscillators ON along with the RMS errors for the sixty-four antennae is shown in Table 6. The data for calibration oscillators OFF along with the RMS errors for the sixty-four antennae is shown in Table 7. And finally the error in $\alpha_{x}$ and $\alpha_{y}$ for all thirty-two pairs of antennae is shown in Table 8 .

A block diagram of the electronics is shown in Fig. 34. A single electron source of 


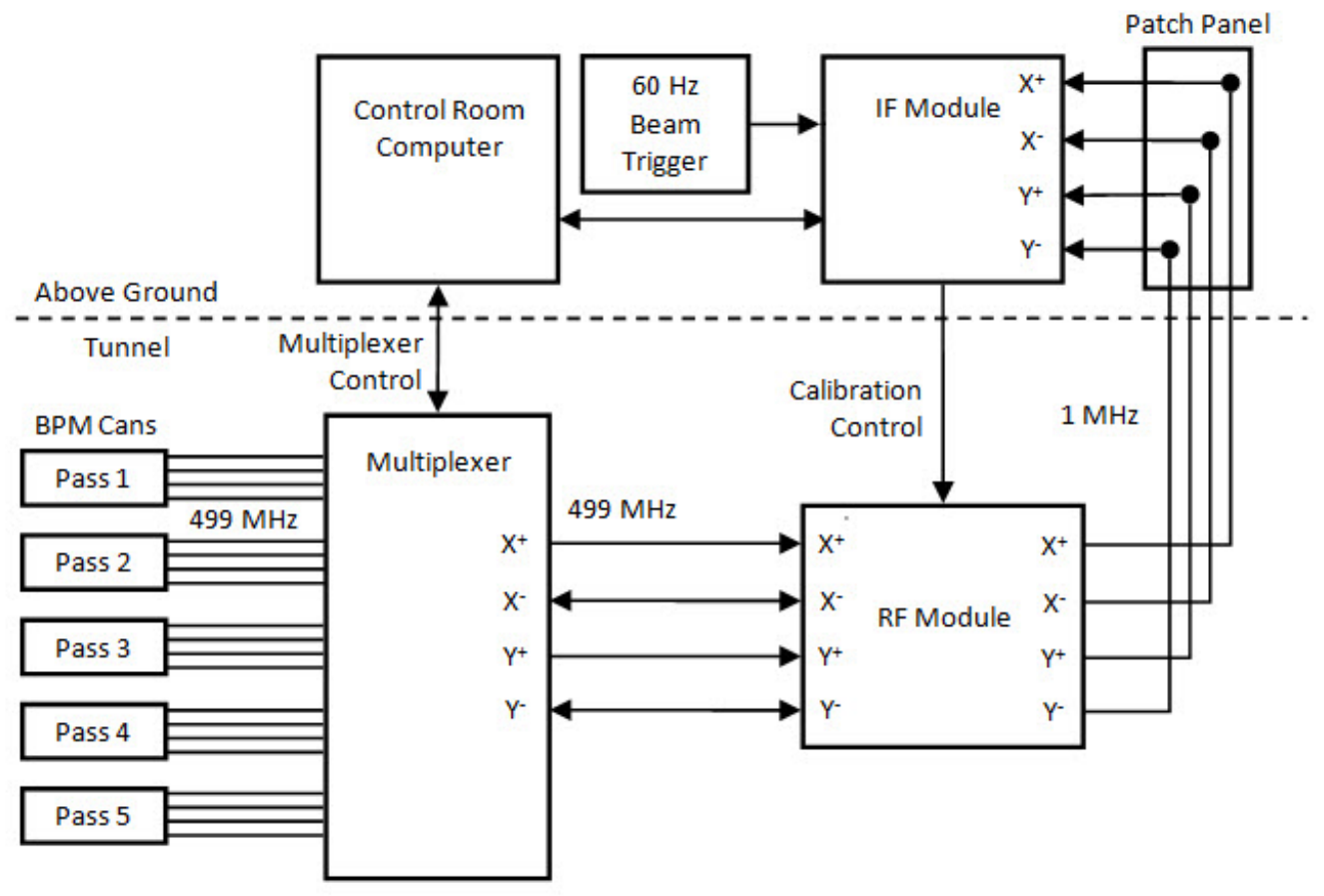

FIG. 34. A schematic layout of the BPM Electronics.

TABLE 6. BPM Wire Data with Calibration Oscillators On

\begin{tabular}{lcccc}
\hline \hline BPM Name & $X^{+}$ & $X^{-}$ & $Y^{+}$ & $Y^{-}$ \\
\hline IPM1A10 & $11799 \pm 48$ & $8939 \pm 26$ & $10872 \pm 32$ & $9147 \pm 61$ \\
IPM1A11 & $8395 \pm 33$ & $8834 \pm 33$ & $8170 \pm 47$ & $8255 \pm 39$ \\
IPM1A13 & $7792 \pm 41$ & $7459 \pm 28$ & $8142 \pm 31$ & $9299 \pm 31$ \\
IPM1A14 & $5668 \pm 56$ & $6719 \pm 40$ & $4713 \pm 39$ & $6571 \pm 29$ \\
IPM1A16 & $6591 \pm 38$ & $6264 \pm 26$ & $5244 \pm 30$ & $5932 \pm 24$ \\
IPM1A18 & $5286 \pm 30$ & $5243 \pm 29$ & $4905 \pm 26$ & $4639 \pm 21$ \\
IPM1A19 & $3177 \pm 19$ & $3135 \pm 18$ & $3644 \pm 41$ & $4246 \pm 24$ \\
IPM1A21 & $5105 \pm 26$ & $5107 \pm 27$ & $5312 \pm 27$ & $5241 \pm 37$ \\
IPM6T00B & $6504 \pm 45$ & $6280 \pm 30$ & $5628 \pm 27$ & $5623 \pm 48$ \\
IPM6T01 & $4196 \pm 34$ & $4913 \pm 37$ & $4714 \pm 57$ & $4357 \pm 37$ \\
IPM6T03 & $9133 \pm 44$ & $8628 \pm 26$ & $8513 \pm 26$ & $7946 \pm 25$ \\
IPM6T06 & $9888 \pm 59$ & $9020 \pm 42$ & $7718 \pm 41$ & $7558 \pm 35$ \\
IPM6T07 & $5428 \pm 55$ & $5488 \pm 25$ & $5670 \pm 32$ & $6095 \pm 27$ \\
IPM6T08 & $4555 \pm 31$ & $3933 \pm 25$ & $3996 \pm 24$ & $4271 \pm 23$ \\
IPM6T09 & $4059 \pm 19$ & $3831 \pm 26$ & $3947 \pm 53$ & $3531 \pm 23$ \\
IPM6T09A & $4511 \pm 28$ & $4993 \pm 28$ & $4967 \pm 31$ & $4679 \pm 41$ \\
\hline \hline
\end{tabular}


TABLE 7. BPM Wire Data with Calibration Oscillators Off

\begin{tabular}{lcccc}
\hline \hline BPM Name & $X_{\text {off }}^{+}$ & $X_{\text {off }}^{-}$ & $Y_{\text {off }}^{+}$ & $Y_{\text {off }}^{-}$ \\
\hline IPM1A10 & $797 \pm 28$ & $210 \pm 14$ & $471 \pm 21$ & $745 \pm 60$ \\
IPM1A11 & $319 \pm 16$ & $459 \pm 16$ & $480 \pm 57$ & $459 \pm 31$ \\
IPM1A13 & $614 \pm 55$ & $390 \pm 37$ & $378 \pm 23$ & $403 \pm 17$ \\
IPM1A14 & $676 \pm 68$ & $431 \pm 25$ & $497 \pm 27$ & $252 \pm 13$ \\
IPM1A16 & $484 \pm 13$ & $224 \pm 28$ & $356 \pm 15$ & $278 \pm 36$ \\
IPM1A18 & $355 \pm 18$ & $494 \pm 13$ & $503 \pm 45$ & $275 \pm 15$ \\
IPM1A19 & $376 \pm 26$ & $260 \pm 20$ & $1156 \pm 29$ & $532 \pm 31$ \\
IPM1A21 & $230 \pm 13$ & $426 \pm 20$ & $278 \pm 19$ & $629 \pm 19$ \\
IPM6T00B & $630 \pm 27$ & $338 \pm 18$ & $264 \pm 17$ & $617 \pm 40$ \\
IPM6T01 & $403 \pm 26$ & $387 \pm 20$ & $690 \pm 73$ & $429 \pm 34$ \\
IPM6T03 & $675 \pm 51$ & $305 \pm 21$ & $274 \pm 25$ & $221 \pm 13$ \\
IPM6T06 & $594 \pm 33$ & $537 \pm 23$ & $413 \pm 49$ & $419 \pm 22$ \\
IPM6T07 & $736 \pm 22$ & $192 \pm 86$ & $381 \pm 31$ & $268 \pm 17$ \\
IPM6T08 & $335 \pm 19$ & $301 \pm 28$ & $262 \pm 18$ & $231 \pm 11$ \\
IPM6T09 & $309 \pm 21$ & $451 \pm 39$ & $869 \pm 24$ & $346 \pm 25$ \\
IPM6T09A & $233 \pm 21$ & $291 \pm 15$ & $353 \pm 19$ & $614 \pm 19$ \\
\hline \hline
\end{tabular}

TABLE 8. Table of $\alpha$ data for all sixteen BPMs.

\begin{tabular}{lcc}
\hline \hline BPM Name & $\alpha_{x}$ & $\alpha_{y}$ \\
\hline IPM1A10 & $1.263 \pm 0.008$ & $1.222 \pm 0.014$ \\
IPM1A11 & $0.964 \pm 0.006$ & $0.989 \pm 0.012$ \\
IPM1A13 & $1.017 \pm 0.012$ & $0.865 \pm 0.006$ \\
IPM1A14 & $0.791 \pm 0.015$ & $0.663 \pm 0.008$ \\
IPM1A16 & $1.019 \pm 0.009$ & $0.871 \pm 0.010$ \\
IPM1A18 & $1.047 \pm 0.010$ & $1.013 \pm 0.013$ \\
IPM1A19 & $0.992 \pm 0.015$ & $0.680 \pm 0.016$ \\
IPM1A21 & $1.044 \pm 0.009$ & $1.069 \pm 0.012$ \\
IPM6T00B & $0.999 \pm 0.011$ & $1.031 \pm 0.015$ \\
IPM6T01 & $0.844 \pm 0.012$ & $1.069 \pm 0.027$ \\
IPM6T03 & $1.026 \pm 0.009$ & $1.070 \pm 0.006$ \\
IPM6T06 & $1.099 \pm 0.010$ & $1.018 \pm 0.011$ \\
IPM6T07 & $0.874 \pm 0.019$ & $0.903 \pm 0.009$ \\
IPM6T08 & $1.171 \pm 0.016$ & $0.920 \pm 0.010$ \\
IPM6T09 & $1.089 \pm 0.017$ & $0.963 \pm 0.021$ \\
IPM6T09A & $0.916 \pm 0.010$ & $1.138 \pm 0.015$ \\
\hline \hline
\end{tabular}


the three laser injector system was used for this experiment which provides beam at $499 \mathrm{MHz}$. A multiplexer sequentially switches five BPMs, each one from a particular pass, to the RF Module located adjacent to the beamline. The RF Module is a 4-channel amplifier that down-converts the RF signal from the BPM to $1 \mathrm{MHz}$ to reduce the transmission loss in the long signal cables that are connected to the patch panel in the above ground Service Building. The RF Module also includes a 2-channel RF calibration source used for determining $\alpha_{x}$ and $\alpha_{y}$.

The signals are connected from the Patch Panel to the IF Module which resolves the $1 \mathrm{MHz}$ signal to a DC level. The signal is then digitized and sent over the network for control room display. The IF module is triggered to acquire data at $60 \mathrm{~Hz}$ for typical beam operations. For this thesis the IF module is replaced with a high speed digital data acquisition system which is described in section 4.4.

\subsection{BEAM MACROPULSE STRUCTURE}

The CEBAF electron beam for a single laser consists of a $499 \mathrm{MHz}$ Continuous Wave of electrons. The average power in this CW beam is simply the average current times the voltage gained through acceleration $\mathrm{P}(\mathrm{W})=\mathrm{I}(\mu \mathrm{A}) \times \mathrm{V}(\mathrm{MV})$. The accelerator is classified as a Mega-Watt beam facility and reached this milestone in 1996 with a $1497 \mathrm{MHz}, 200 \mu \mathrm{A}, 4 \mathrm{GeV}$ beam as was shown in Table 2. During machine tuning, and beam modulation experiments such as this, the total power must be limited to prevent the beam from melting through the stainless steel beam tube at any location of beam loss.

To have an acceptable signal:noise ratio for the BPM system, a reasonable minimum threshold current is around $10 \mu \mathrm{A}$. This gives $10 \mathrm{~W} / \mathrm{MeV}$ which is still too high for the typical energies of the machine. The duty factor of the beam must be reduced to lower the average power. The nominal tuning beam has the pulse structure shown at the top in Fig. 35 and consists of a $60 \mathrm{~Hz}$ train of $250 \mu$ s macropulses with an average current of $10 \mu \mathrm{A}$ within the macropulse. Beyond the main macropulse is a $4 \mu \mathrm{s}$ long trailing pulse used by the linac BPMs to detect the time of flight separated signals of the different passes within the linac. The duty factor for this configuration is $1.5 \%$.

The pulse structure chosen for the modulation experiment is shown at the bottom in Fig. 35 and consists of a $500 \mathrm{~Hz}$ train of $100 \mu$ s macropulses with an average current of $10 \mu \mathrm{A}$ within the macropulse. The duty factor for this configuration is $5 \%$. This 

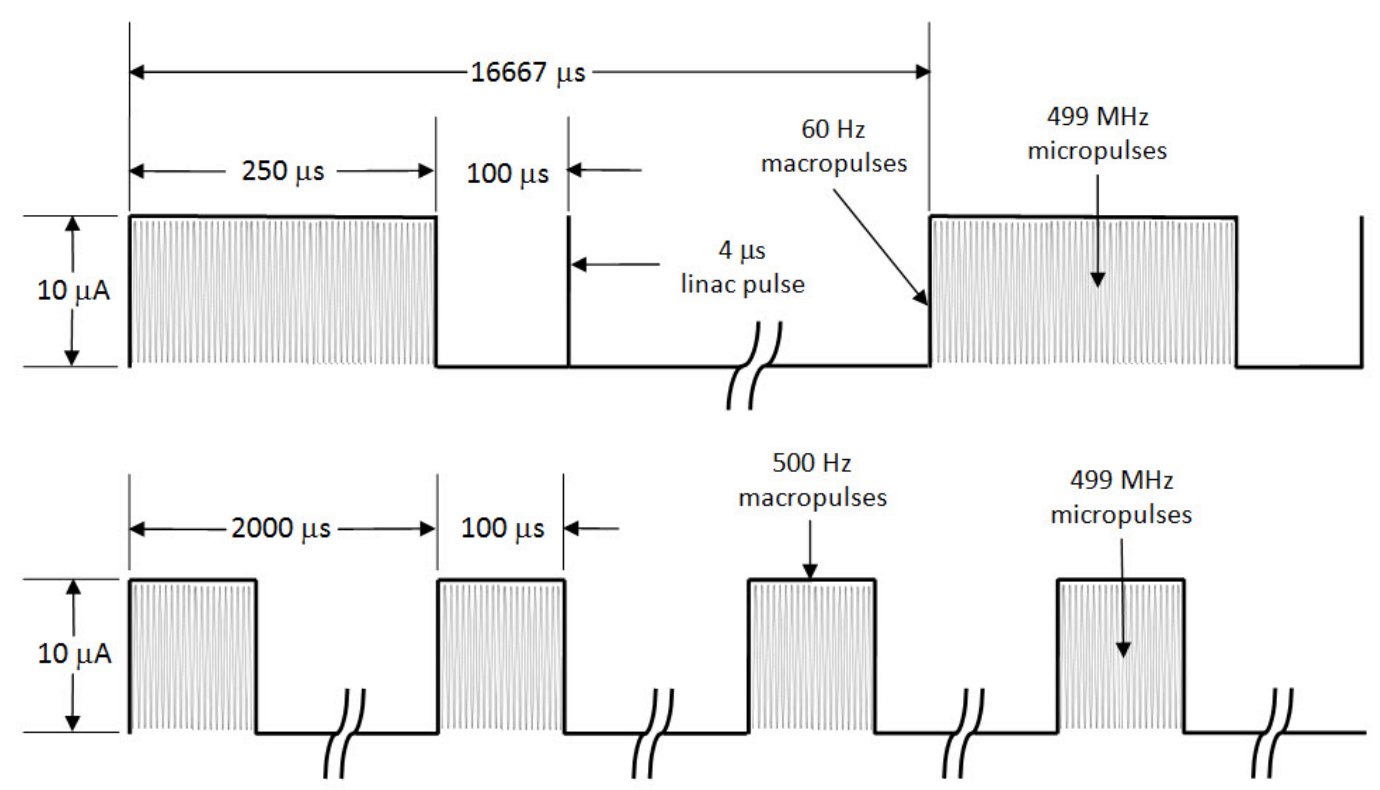

FIG. 35. The beam pulse structure used for tuning the machine is shown at the top. The beam pulse structure during modulation experiments is shown at the bottom.

particular pulse structure was selected for the following reasons:

1. The dominant noise source affecting the beam comes from $60 \mathrm{~Hz}$ power supply fluctuations. These are significant out to the 4th harmonic or $240 \mathrm{~Hz}$. To keep track of these errors the sample rate has to be at least twice this frequency or $480 \mathrm{~Hz}$ per the Nyquist theorem.

2. The insertable beam dumps in the accelerator have a power limit of $2 \mathrm{~kW}$. For the energies used in this experiment this corresponds to $311 \mathrm{~W}$ and $1711 \mathrm{~W}$ in Arc 1 and the Arc 6 Recombiner respectively.

3. The signal to noise ratio and the ability to resolve the peaks in the BPM spectrum are improved with more samples.

4. The data acquisition system uses I-Q sampling (described in section 4.4) to obtain the beam position from the down converted $1 \mathrm{MHz}$ BPM signals. Within the $100 \mu$ s macropulse there are then a hundred $1 \mathrm{MHz}$ cycles that can be averaged to improve the data quality. 


\subsection{DATA ACQUISITION SYSTEM HARDWARE}

The data acquisition system used in this thesis was initially developed as a prototype system for detecting fast transients in the linac RF during beam trips [20]. This Distributed Data Acquisition System (DDA) is a modular design consisting of a main motherboard using an embedded IOC based on the PC/104 architecture. The computer runs EPICS (Experimental Physics and Industrial Control System) on top of RTEMS (Real-Time Executive for Multiprocessor Systems) software. This single board computer can take up to five data acquisition modules that are each capable of receiving twelve channels. A picture of the prototype installed in the service building during the experiment is shown in Fig. 36. This experiment used three of the data acquisition modules, visible along the front of the chassis, for a total of thirty-six inputs. The BPM patch panel is also visible in the photo at the lower right side. On top of the chassis is a multi-output DC source for powering the system.

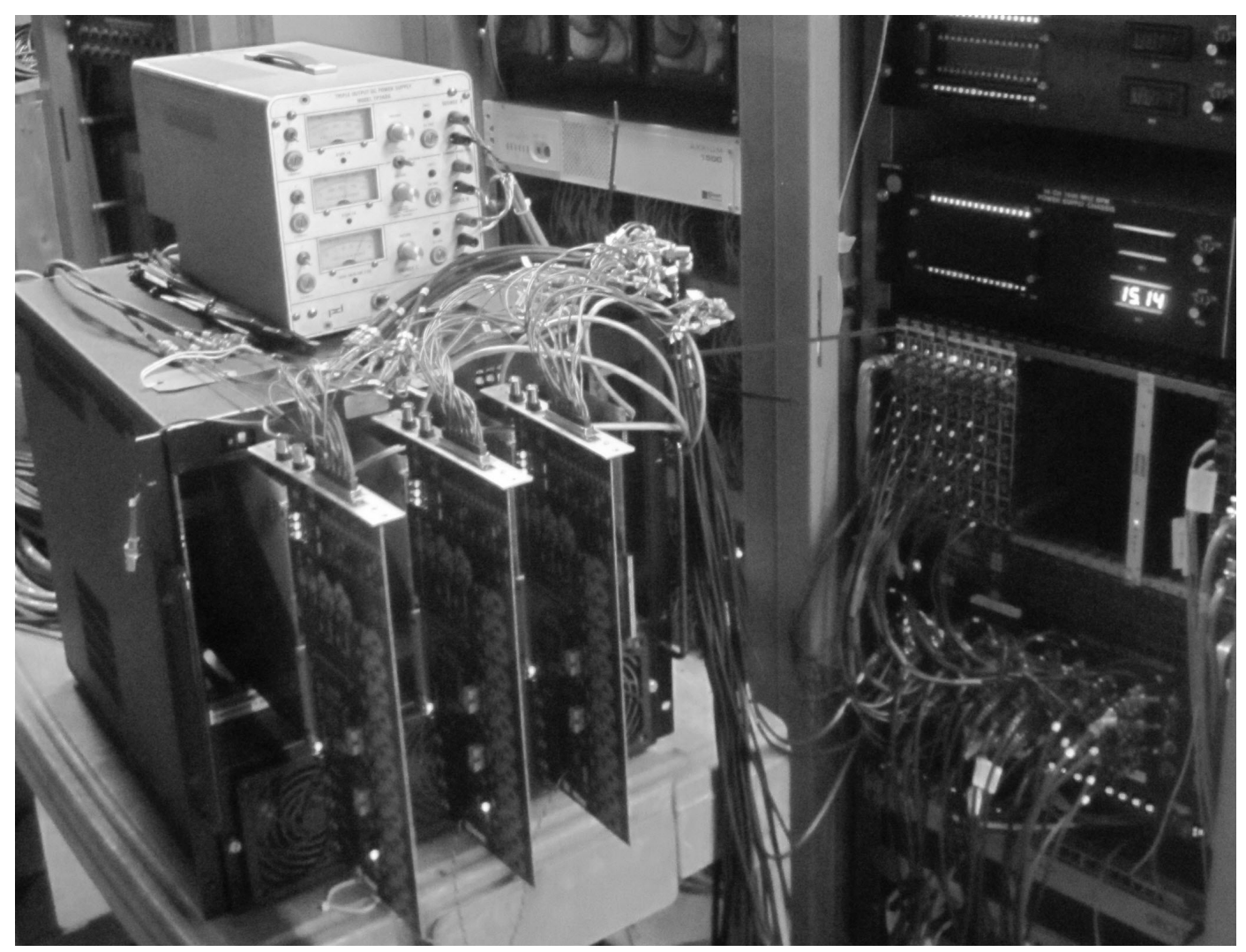

FIG. 36. A picture of the data acquisition system in the service building. 


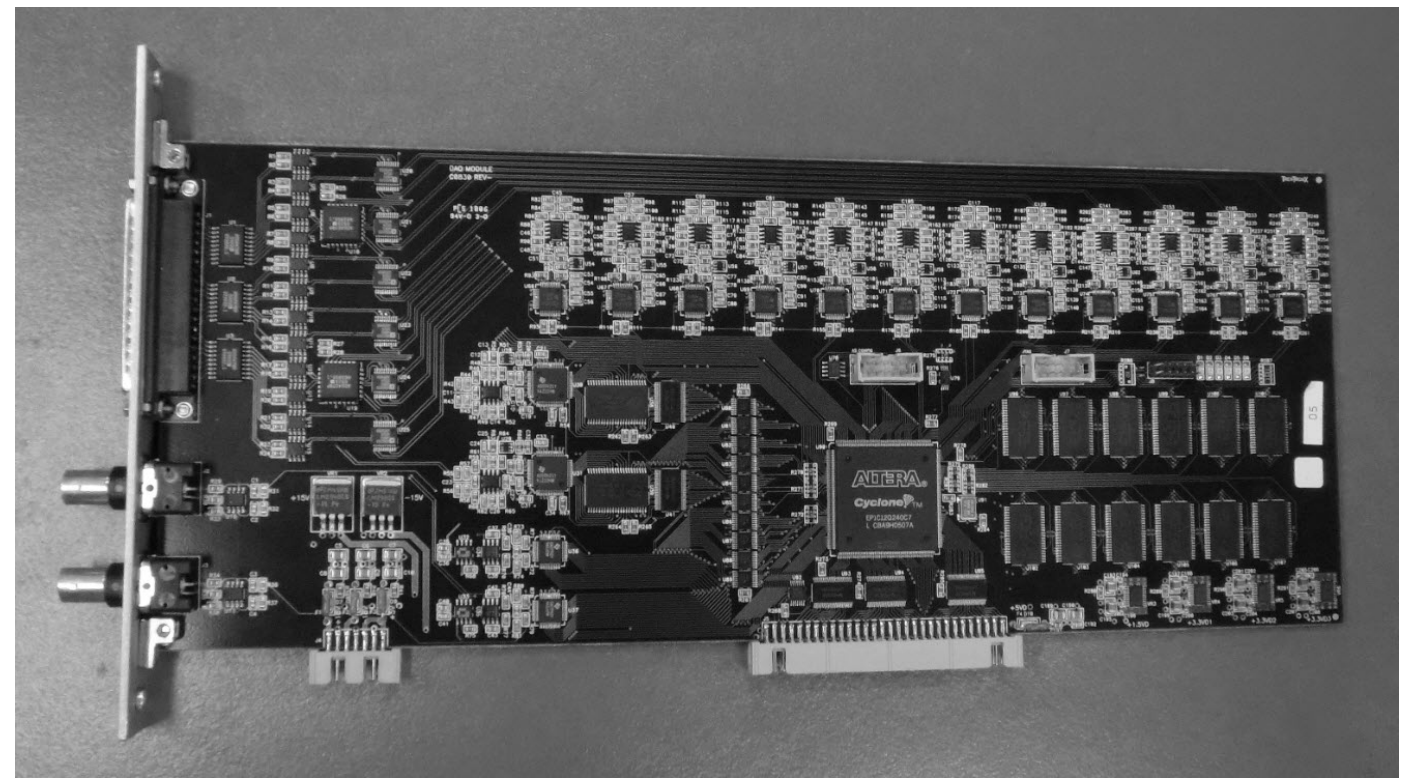

FIG. 37. A picture of one of the four data acquisition circuit boards.

The first thirty-two channels were used for the $1 \mathrm{MHz}$ RF signals from the eight 4-wire BPMs. The BPM patch panel can been seen at the lower right in the photo. The last four channels were used to record the $1 \mathrm{~Hz}$ and $21 \mathrm{~Hz}$ signals from the four kicker magnets. A picture of one of the boards is shown in Fig. 37. The twelve ADCs can be seen along the top of the board with a Field Programmable Gated Array (FPGA) and memory registers at the bottom right.

A schematic representation of the ADC data flow is shown in Fig. 38. The thirty-two $1 \mathrm{MHz}$ signals from the BPM system were AC coupled to a buffering amplifier with a $1.2 \mathrm{MHz}$ bandwidth and an expected input voltage of $\pm 1 \mathrm{~V}$. The two $1 \mathrm{~Hz}$ and two $21 \mathrm{~Hz}$ signals from the kicker magnets were also AC coupled to

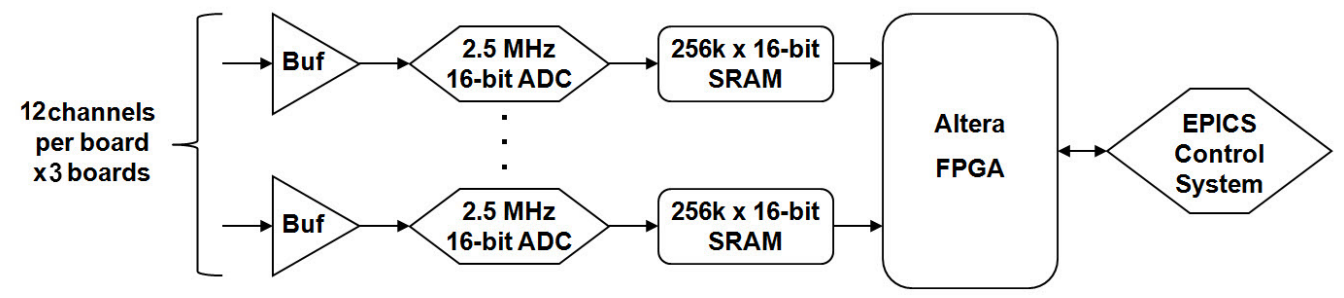

FIG. 38. A schematic representation of the data flow through an ADC board. 

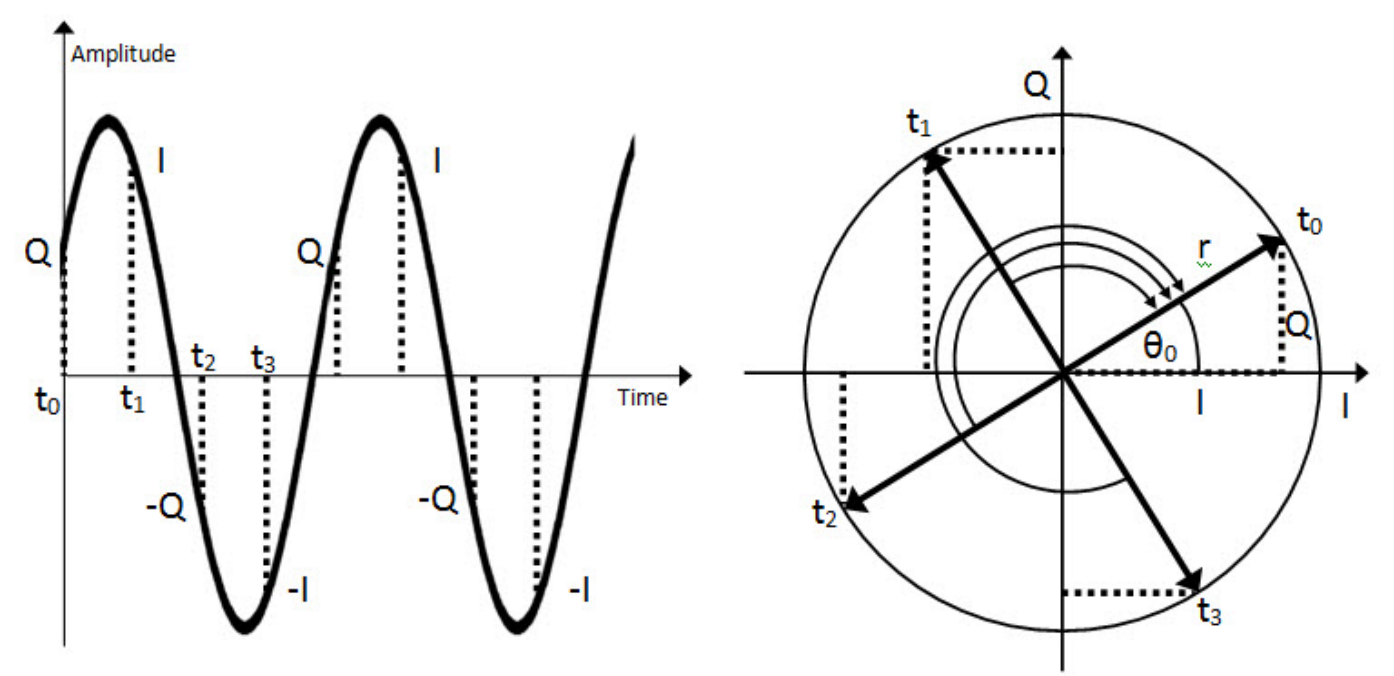

FIG. 39. $I-Q$ sampling as a function of time shown at the right. A phasor diagram showing the same sampling is shown at the left.

the buffering amplifier but with a modified gain resistor to account for the expected $\pm 10 \mathrm{~V}$. The signals are then sampled with a $2.5 \mathrm{MHz} 16$-bit ADC and passed to an FPGA through a circular buffer. A $30 \mu$ s delay relative to the trigger was used to reject any beam-loading transients on the leading edge of the $100 \mu$ s pulse.

During the remaining $70 \mu$ s pulse each kicker signal was acquired 175 times and then averaged on the FPGA to provide one data point per pulse over the EPICS network. The method of acquiring the much faster $1 \mathrm{MHz}$ BPM data for this experiment relies on $I$ - $Q$ sampling and an algorithm called CORDIC [21] which stands for Coordinate Rotation Digital Computer. The algorithm is coded on the FPGA.

An arbitrary RF signal can be represented by

$$
y(t)=A \sin \left(\omega t+\theta_{0}\right)
$$

Rewriting this equation using the addition of sines formula we have

$$
y(t)=A \cos \theta_{0} \sin \omega t+A \sin \theta_{0} \cos \omega t
$$

Now the in-phase component is along the $x$-axis and the quadrature component is along the $y$-axis as seen in Fig. 39. The abitrary RF signal can now be written as

$$
y(t)=I \cos \omega t+Q \sin \omega t .
$$


The $I$ and $Q$ samples are typically derived by phase shifting the signal by $90^{\circ}$ in hardware and then simultaneously sampling both singles to get $I$ and $Q$. The inphase component $I$ and the quadrature component $Q$ are just the axes of the phasor diagram in Fig. 39. The magnitude and phase of the RF signal can now be associated with the values of $I$ and $Q$ and are written as

$$
\begin{gathered}
r=\sqrt{I^{2}+Q^{2}}, \\
\theta=\arctan \left(\frac{Q}{I}\right) .
\end{gathered}
$$

Harmonic sampling of the RF signal can be accomplished using any odd multiple of the original $1 \mathrm{MHz} R F$ signal to yield the required $I-Q$ samples as the phasor rotates through the four quadrants. For example consider the case where $n=2$ which gives a sampling frequency of $800 \mathrm{~Hz}$ as shown in Fig. 40. For this case we have

$$
\frac{4 \times 1 \mathrm{MHz}}{2 n+1}=\frac{4 \times 1 \mathrm{MHz}}{2(2)+1}=800 \mathrm{~Hz} .
$$

This sampling scheme is illustrated in Fig. 40. At the leading edge of the first 800 ksps pulse we pick up an $I^{+}$and the next pulse picks up a $Q^{+}$. The next $I-Q$ pair are negative and inverted to match the previous pair. In this way the data is sampled and for each $I$ - $Q$ pair we can calculate the magnitude and phase using CORDIC as described below.

The ADC samples the RF signal at 2.5 MHz. The FPGA picks out every third data point to create a data stream at $833 \mathrm{ksps}$. The error in sampling frequency relative to $800 \mathrm{ksps}$ produces a small ripple on the sampling stream but during the $70 \mu$ s we acquire $30 I-Q$ pairs which can be averaged. The data quality is further

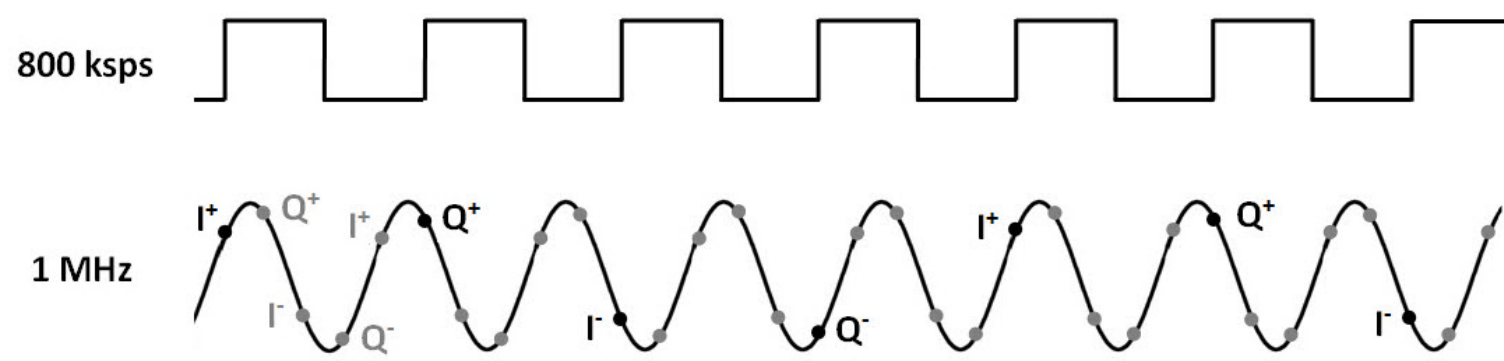

FIG. 40. A drawing showing the $I-Q$ sampling scheme used by the data acquisition system. 


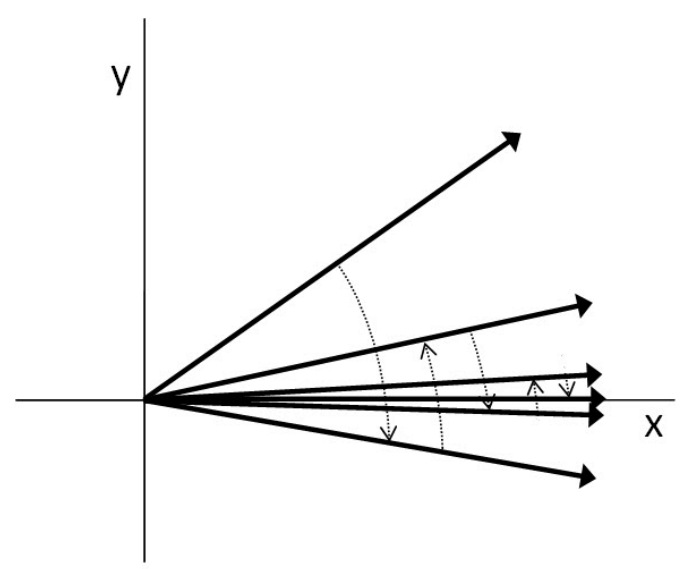

FIG. 41. Illustration of binary search pattern for finding magnitude and phase.

improved by simultaneously using all three 833 ksps streams of interleaved data which provides $90 I$ - $Q$ samples. These are then averaged to provide one number for each $100 \mu$ s beam macropulse.

The CORDIC algorithm uses an iterative binary search to calculate the magnitude and phase of each $I$ - $Q$ sample which is illustrated in Fig. 41. The initial angle of the vector is rotated by 45 degrees. The next rotation is by half the angle; in the same direction if the value of $y$ is still positive or in the opposite direction if $y$ is negative. This repeats until the resultant lies on the $x$ axis. Adding all of the individual angles recovers the value of the initial angle and the magnitude is now just the value of $x$.

The search is made into a binary search by using powers of 2 to define the angles

TABLE 9. Values of CORDIC angles for binary search.

\begin{tabular}{ccccc}
\hline \hline Angle & TAN(Angle) & i & Nearest $2^{-i}$ & ATAN $\left(2^{-i}\right)$ \\
\hline 45 & 1 & 0 & 1 & 45 \\
22.5 & 0.414 & 1 & 0.5 & 26.57 \\
11.25 & 0.199 & 2 & 0.25 & 14.04 \\
5.625 & 0.0985 & 3 & 0.125 & 7.125 \\
2.8125 & 0.0491 & 4 & 0.0625 & 3.576 \\
1.40625 & 0.0245 & 5 & 0.03125 & 1.790 \\
0.703125 & 0.0123 & 6 & 0.015625 & 0.8952 \\
\hline \hline
\end{tabular}


as indicated in Table 9. In this sense the original angle is then given by

$$
\theta=\sum_{i} d_{i} \arctan \left(2^{-i}\right)
$$

where $d_{i}=+1$ if $y_{i}<0$ and $d_{i}=-1$ if $y_{i} \geq 0$. 


\section{CHAPTER 5}

\section{LABORATORY MEASUREMENTS AND SIMULATIONS}

Results from laboratory measurements and off-line simulations that were done in support of the thesis are presented in this chapter. To accurately measure the nonlinearities of the magnetic fields one needs to modulate the beam to large amplitudes where the BPMs are also nonlinear. The linearization of the BPM system is presented in section 5.1. The nonlinearity of the Arc $1 \mathrm{BE}$ dipoles was measured in the lab and simulated in software before measuring with the beam. In section 5.2 we present the multipole data from the Magnet Measurement Facility and in section 5.3 we provide the results of Tosca simulations of these same dipoles. Elegant particle tracking code was used to simulate the beam transport across a sextupole magnet and a dipole magnet. The results are presented in section 5.4.

\subsection{BPM NONLINEARITY CORRECTION}

The difference/sum method assumes that the BPM response is linear across the whole range through the linearity constant $k$. Early measurements [19] of the CEBAF M15 and M20 BPMs have shown that the devices are linear within the \pm 5 $\mathrm{mm}$ aperture that the beam is typically held within. This research requires large amplitude orbit excitation to explore the nonlinearities of the magnets. Under these conditions the BPM nonlinearity must be corrected.

A surface wave transmission system had been developed at Jefferson Lab [22], along with a precision translation stage, to perform in-air tests on the M15 and M20 BPMs (see Fig. 42).

It was demonstrated by Sommerfeld [23] that certain dielectric boundary conditions allow for the existence of a traveling wave on the surface of a coaxial cylinder with finite conductivity. Goubau [24] first proposed a method for launching and capturing these waves as a substitute for low-loss coaxial microwave transmission systems. The Goubau Line (G-Line) system consists of a single thin conductor coated in a dielectric material. The wire is connected to conical launchers that excite the proper fields for standing-wave formation. The launchers also provide impedance matching from the $50 \Omega$ transmission line to the nominal $200 \Omega$ of the thin conductor. 


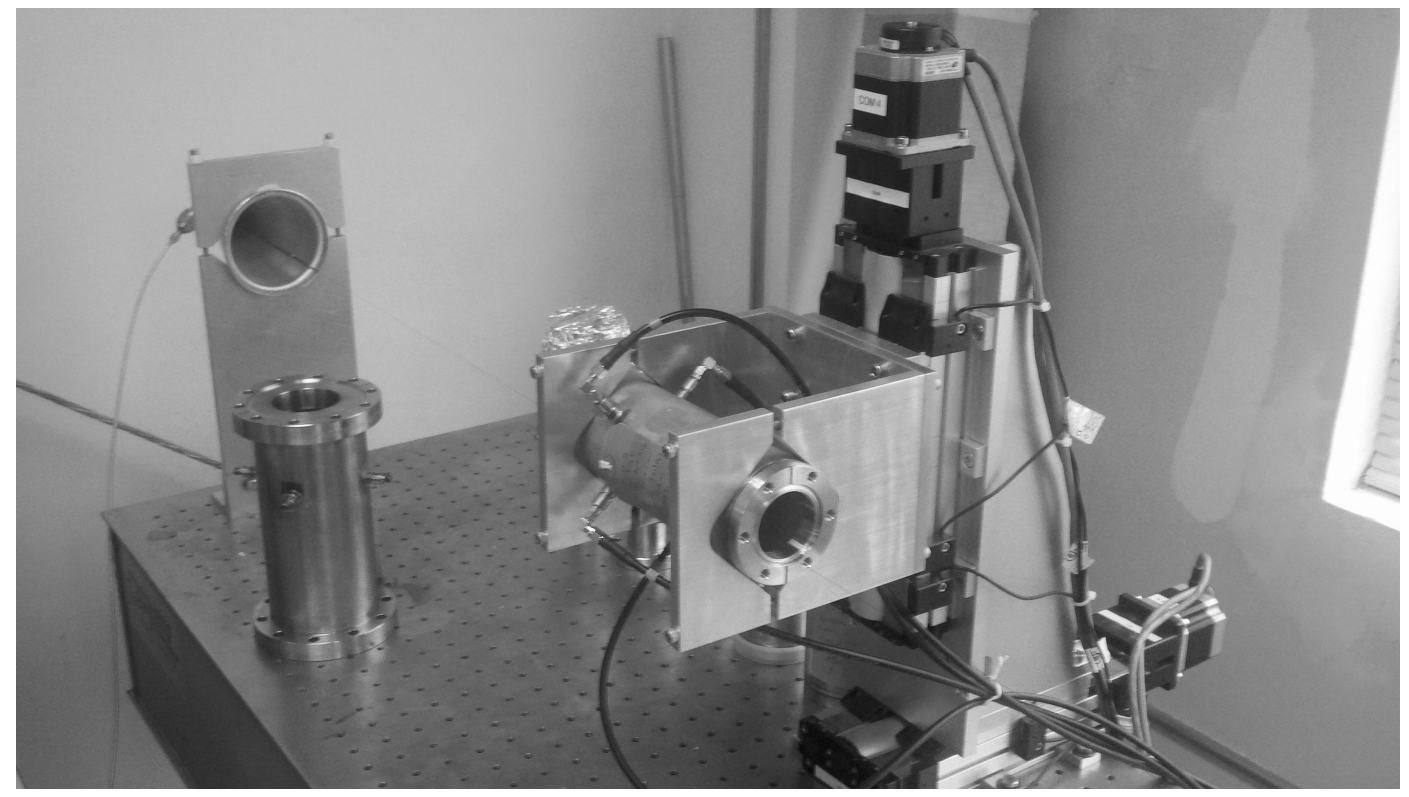

FIG. 42. A picture of the BPM test stand with an M15 BPM mounted on the stage and an M20 BPM sitting on the table.

The electron beam is simulated with a 34 AWG enameled magnet wire having a diameter of 160 microns. This is comparable to typical beam sizes in CEBAF. The wire is passed through the BPM can and then soldered to the center conductor of the cones at either end of the the test stand. One end of the wire is terminated in a $50 \Omega$ load with the other end connected to an RF source. The wire is held under tension within the BPM to properly simulate a beam. The BPM under test was oriented with the $X^{+}$and $X^{-}$antennas oriented in the horizontal plane. The translation stage was then moved in 200 micron steps from $+21 \mathrm{~mm}$ to $-21 \mathrm{~mm}$. The raw wire data at each step was processed using the difference/sum method and shows that the system behaves linearly to about $\pm 8 \mathrm{~mm}$ as shown at the left in Fig. 43 .

To correct for the BPM nonlinearity we follow a method used at Fermilab [25]. A two-dimensional electrostatic model of the M15 and M20 BPMs was developed using Poisson [26]. A potential of 1 Volt was placed on a single electrode with the outside of the can grounded. The potential map was calculated across the interior of the BPM and is shown as a contour plot at the right in Fig. 43. Using Green's reciprocity theorem [27] we can infer that the simulated voltage at any point within the BPM is simply the voltage that would be induced on the antenna. Potential maps for the other three antennae are generated through rotations using the inherent symmetry 

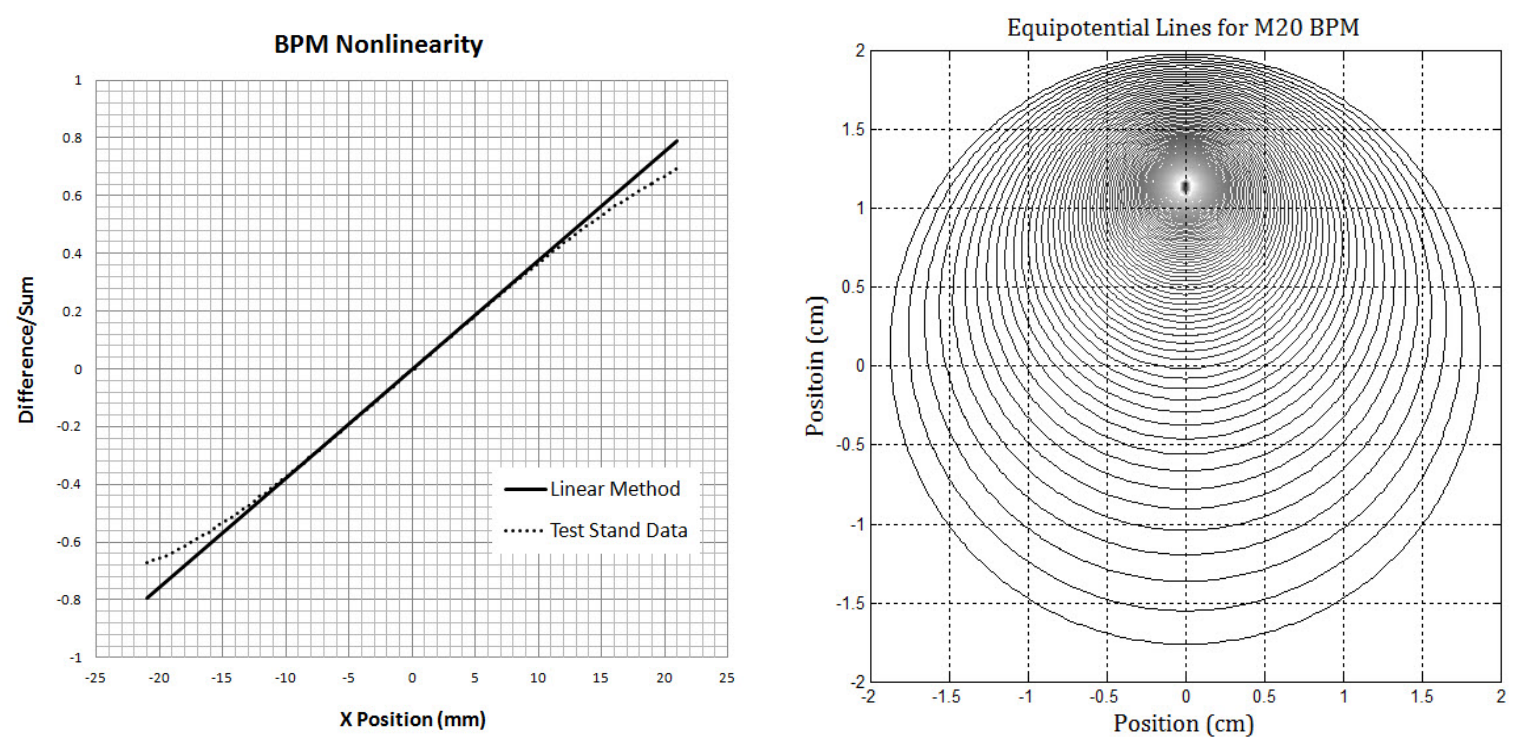

FIG. 43. Data from the BPM test stand showing the nonlinear response and the nonlinear potential map that was generated using Poisson.

of the BPM. The input file for generating the electrostatic model for an M20 BPM is shown in Appendix C.

The Poisson model was seeded with a dense square grid of points across the full aperture of the BPM to simulate the nonlinearity. The points are shown in the upper left graph of Fig. 44. For each point within the grid a spline interpolation was performed to calculate the potential on each wire based on the Poisson model. The difference/sum method was then applied using the nominal values for $k$ to create a 2dimensional map of what would be measured with the linear method. The simulations were done in the rotated frame which places the antennae at the top, bottom, left and right of the grid. Significant pin cushioning of the linear map is observed in the upper right graph of Fig. 44.

A correction of the distortion is made by generating a pair of two-dimensional polynomials. The square grid of points and the values from the linear method are used to calculate the coefficients in a least squares sense and then applied to the distorted position map. The corrected grid of points is shown in the lower left part of the same figure. The precision of the correction is gauged by plotting the absolute value of the difference between the square grid of points and the corrected grid of points. The method recovers the original grid to better than 100 microns across the entire grid of points. 

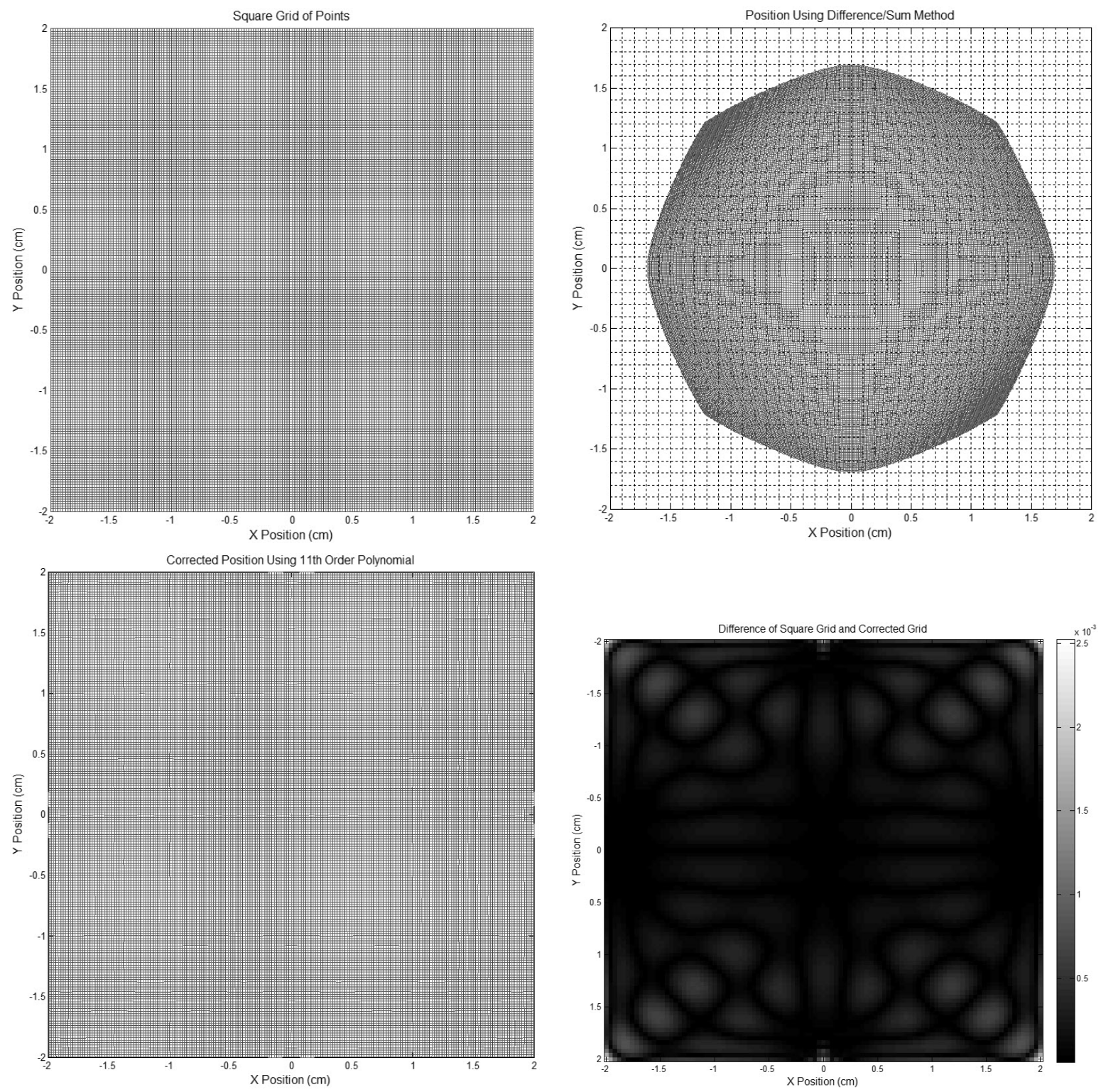

FIG. 44. A sequence of plots showing the distortion of BPM data due to the limitations of the difference/sum method and the results of the correction. 

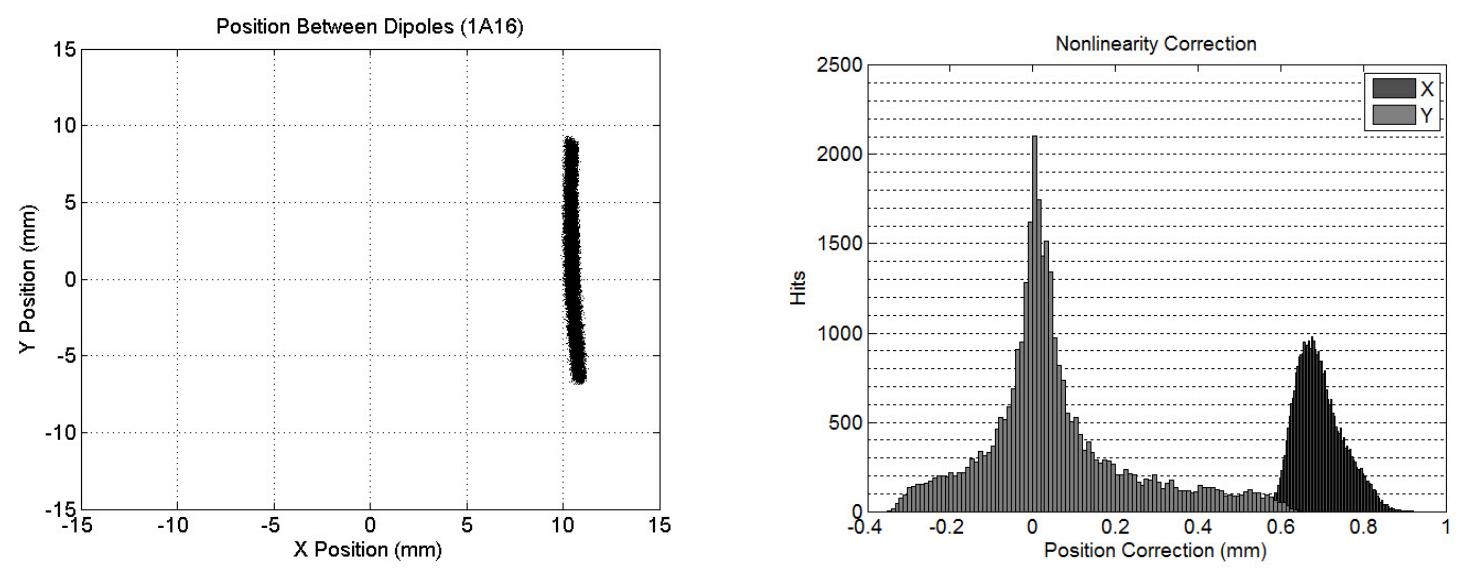

FIG. 45. Beam modulation in $y$-plane at left and a histogram of position corrections at right.

An example of the correction algorithm is shown in Fig. 45. At the left is a plot of the beam position in the $x-y$ plane with the beam centroid displaced in $x$ and modulated in $y$. A histogram of the corrections is shown at the right. The $x$ corrections are centered at 650 microns with the distribution biased towards the right because of the slight curvature of the trace in the $x$ direction. The $y$ corrections are centered near zero and also have a small bias towards more positive values because the distribution is not quite centered about $y=0$.

\subsection{BE DIPOLE LAB MEASUREMENTS}

All of the magnets that are installed in the CEBAF machine are initially qualified at our Magnet Measurement Facility. Multiple techniques are used to measure the field quality of the magnets relative to design specifications. These include Moving Stretched Wire, Rotating Coil, as well as a Hall Probe Stepper Stand. The latter was used to measure one of the meter long Arc 1 BE dipoles with data analyzed on curved beam trajectories [28, 29].

The magnetic field in the vertical direction $\left(B_{y}\right)$ was collected using a Hall probe. The probe was stepped through the magnet along the midplane in a grid pattern along the $x$ and $z$ directions. The longitudinal $z$ steps went from $0.5 \mathrm{~m}$ outside of the steel to the longitudinal midpoint of the $1 \mathrm{~m}$ magnet in steps of $0.2 \mathrm{~cm}$. The probe was then shifted in $x$ for another run in $z$. The transverse $x$ steps went from $+5 \mathrm{~cm}$ to $-5 \mathrm{~cm}$ in $0.5 \mathrm{~cm}$ steps with the zero aligned to the center of the pole width. 


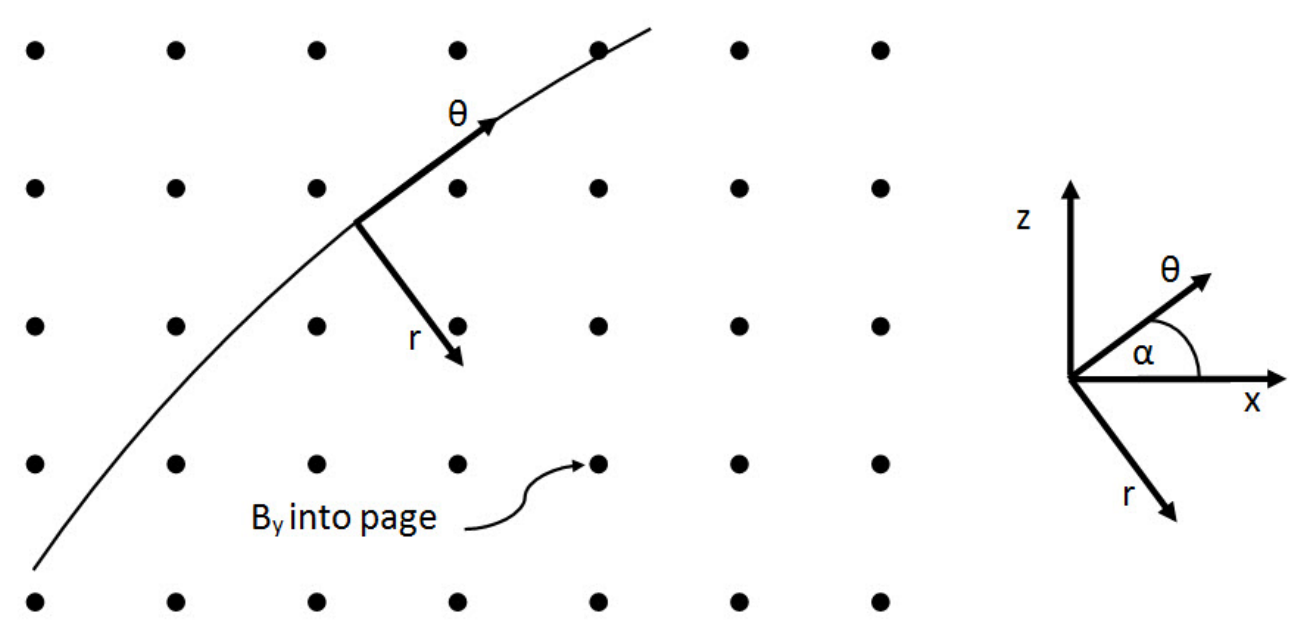

FIG. 46. A sketch of the grid of points and the coordinate system for calculating fields along a curved trajectory.

The integrated dipole field strength is calculated along a trajectory defined by the design bend radius of the magnet. The ideal path is derived from optics modeling software. Along this curved trajectory $s$ a fit is made every $0.5 \mathrm{~cm}$ using the local grid of points to determine an interpolated field point (see Fig. 46). The data are then integrated along the whole path $s$ and multiplied by two, since only half of the magnet was measured, to get the total dipole strength.

To determine the first order quadrupole term the raw grid of data are used to create a grid of derivative data at each point in the two dimensional map. The derivatives are written as

$$
\begin{aligned}
& \frac{d B}{d x}=\left(B_{x-1}-B_{x+1}\right) / \Delta x, \\
& \frac{d B}{d z}=\left(B_{z-1}-B_{z+1}\right) / \Delta z .
\end{aligned}
$$

For points along the curved trajectory defined by the design bend radius of the magnet the local derivative is calculated using interpolation of the grid of derivative data. The interpolated points are then rotated into the $r, \theta$ coordinates system at fixed radius and integrated. The field gradient along the $\theta$ direction is

$$
\frac{d B}{d \theta}=\frac{d B}{d x} \cos \alpha+\frac{d B}{d z} \sin \alpha
$$

This process of calculating the dipole and quadrupole strength of the magnet is repeated for trajectories shifted in $r$ about the design trajectory from $+2.0 \mathrm{~cm}$ to 


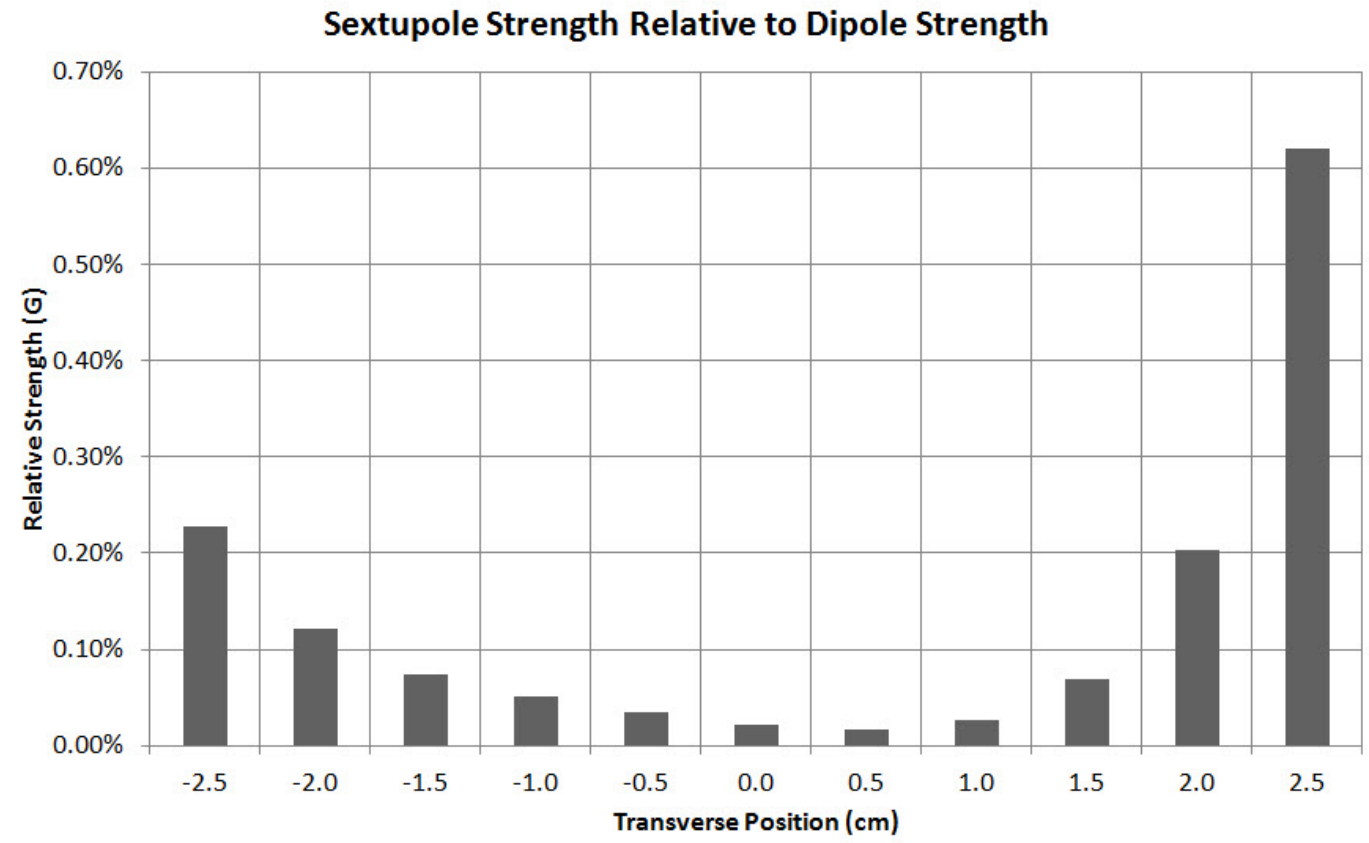

FIG. 47. A plot of relative sextupole strength vs. transverse position for a BE dipole.

$-2.0 \mathrm{~cm}$ in $0.5 \mathrm{~cm}$ steps. The sextupole strength as a function of transverse position is derived by computing $d^{2} B / d r^{2}$ from the family of radial gradient data (Fig. 47).

\subsection{TOSCA ANALYSIS}

The TOSCA 3-D modeling software from Vector Fields was used to predict the sextupole strength of an Arc 1 BE dipole as a function of transverse position within the midplane as well as points above the midplane [30]. A model of the 1-meter dipole magnet is shown in Fig. 48. Throughout the path of the beam, the model uses $1 \mathrm{~mm}$ tetrahedra and quadratic interpolation between the nodes to calculate the field. There are over thirty-three million elements and over forty-one million nodes that are used to model the magnet.

The simulations were done for eighteen different trajectories through the dipole magnet which are shown in Fig. 49. The trajectories start well outside the magnet where the calculated fields are negligible and end at the longitudinal midplane. On each of the overlapping $3 \mathrm{~mm}$ circles the magnetic field $B_{y}$ is calculated at eighteen different points. Fourier analysis is used to compute the multipole content at that location using 

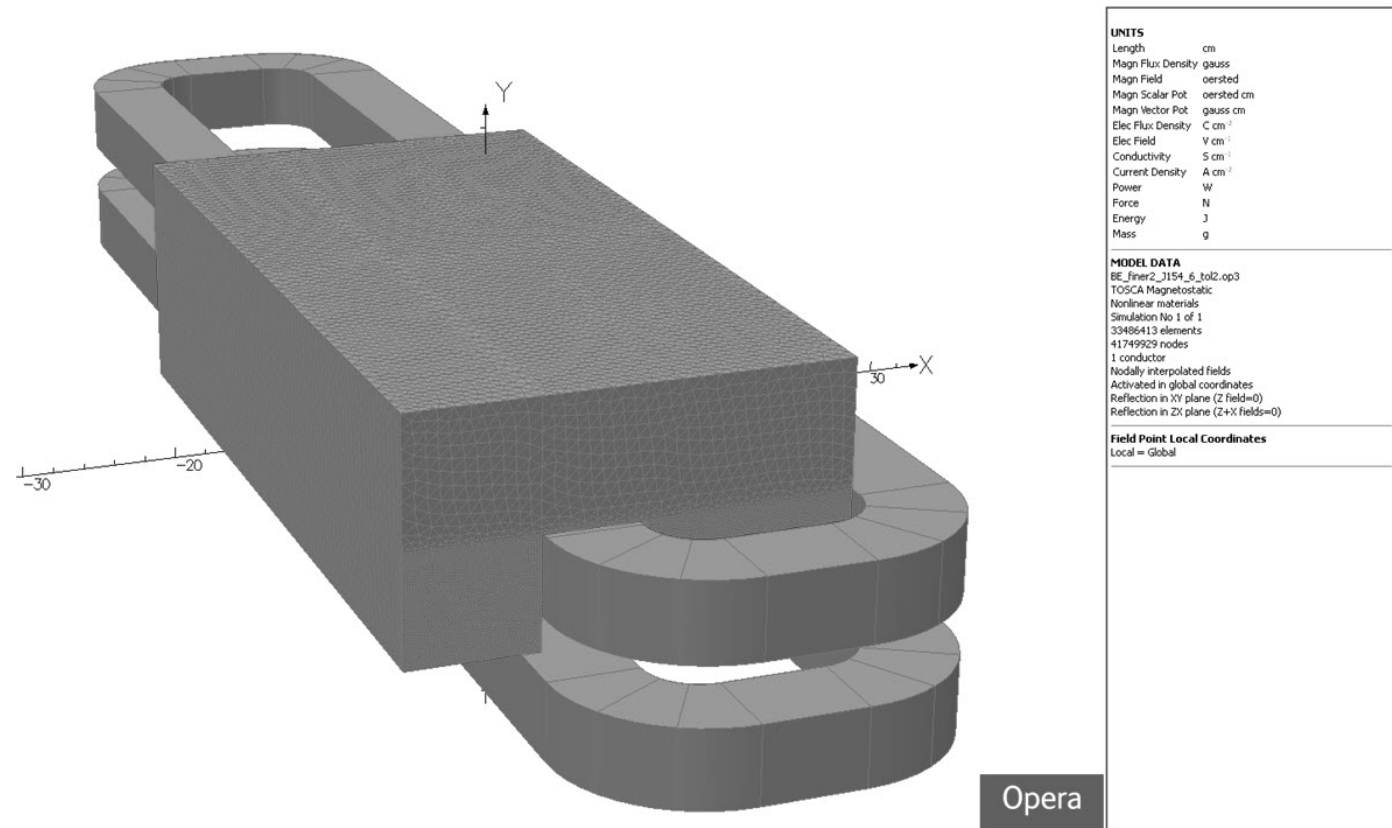

FIG. 48. The 3-D Tosca model of a CEBAF BE-style dipole. The iron above the midplane and the pair of coils are shown.

$$
B_{y}=\sum_{n}^{5}\left(A_{n} \cos (n \theta)+B_{n} \sin (n \theta)\right) .
$$

The $B_{n}$ terms are the skew moments described in section 3.1 and are all zero in the expansion for an upright dipole. The $A_{n}$ are the normal modes with $n=0$ for the dipole term, $n=1$ for the quadrupole term and $n=2$ for the sextupole term.

The simulation shifts along the modelled beam trajectory by $\Delta s=1 \mathrm{~mm}$ and repeats the fourier decomposition for each point. The results are integrated along the path and then multiplied by 2 because only half of the magnet is included in the model. The data is then divided by 10 to get integral $\mathrm{cm}$ instead of $\mathrm{mm}$. The results for the sextupole term are presented in Fig. 50.

The results from the figure show that the sextupole strength increases as the trajectory shifts horizontally towards the open end of the dipole magnet. This is due to the increasing edge effects as we get towards the end of the steel. On the other hand the results indicate that as we shift vertically away from the midplane the sextupole term decreases. This is due to the trajectory getting closer to the pole face of the magnet where the fields are less curved. 


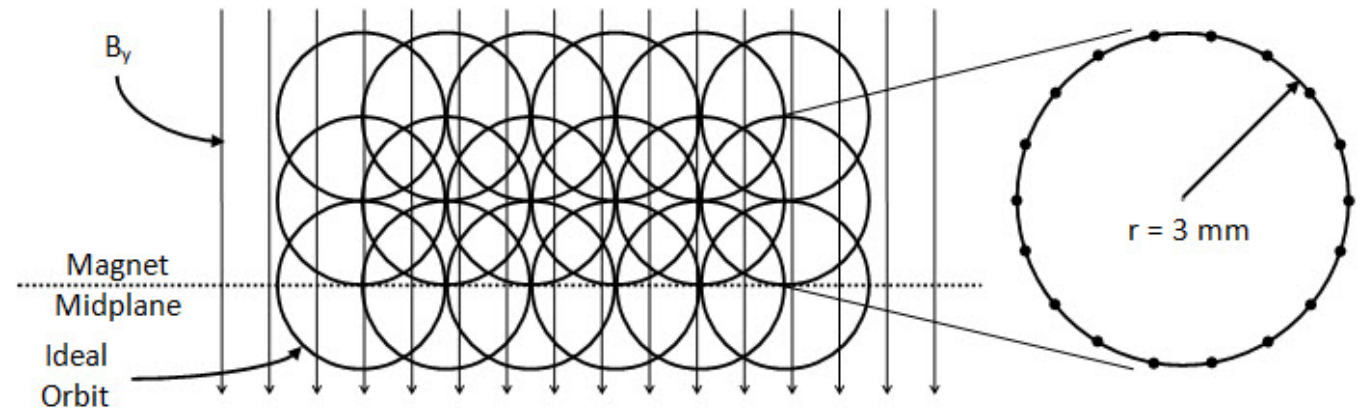

FIG. 49. The arrangement of the eighteen orbits through the BE dipole and the eighteen points around the circle used for TOSCA analysis.

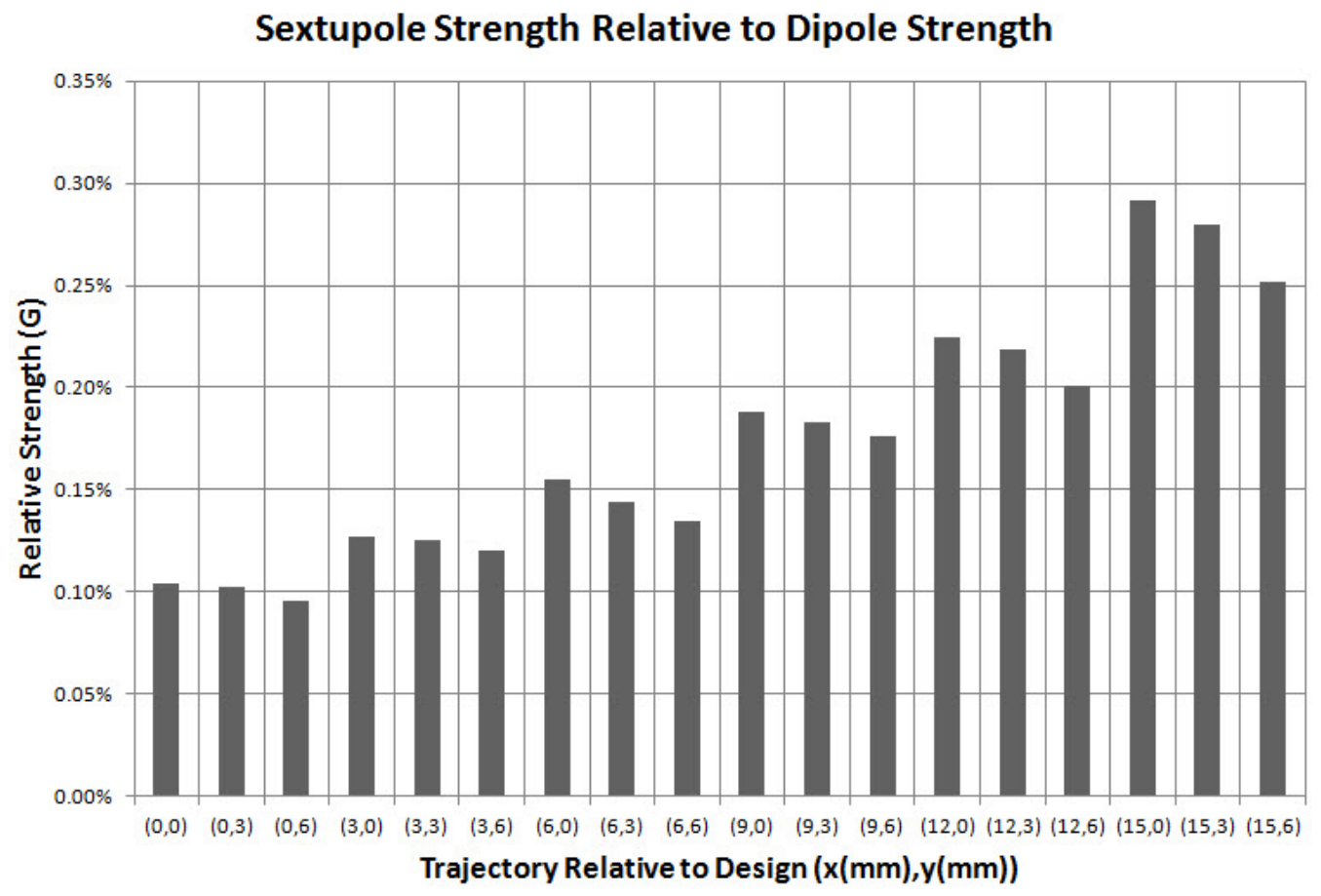

FIG. 50. The integrated relative sextupole strength is plotted vs. the index of the start position. 


\subsection{ELEGANT SIMULATIONS}

Simulations of the ARC1 sextupole measurements and BE dipole measurements were conducted with a particle tracking code, developed at Argonne National Laboratory, called elegant which stands for "ELEctron Generation ANd Tracking". The software is capable of tracking the trajectory of electrons in the 6-dimensional phase space $\left(x, x^{\prime}, y, y^{\prime}, s, \delta\right)$ where $x$ and $x^{\prime}$ are the horizontal transverse coordinate and angle, $y$ and $y^{\prime}$ are the vertical transverse coordinate and angle, $s$ is the total distance travelled and $\delta$ is the fractional change in the electron momentum. The tracking for this study was performed using second order matrices to account for the nonlinear fields of the sextupole and the multipole error of the simulated dipoles.

A model of the beamline was created which includes all elements from the first kicker magnet (MAZ1S08H) to the last BPM in the data acquisition system (IPM1A21). The so called lattice file is shown in Appendix A.1 with the individual beamline elements described below.

The quadrupole magnets in the machine are modeled by the KQUAD element. They are described by their length $L$ in meters and by their geometric quadrupole strength $K 1$ in units of $1 / \mathrm{m}^{2}$ which is defined as

$$
K 1=\frac{.29979 \times g(\mathrm{~T} / \mathrm{m})}{p(\mathrm{GeV} / \mathrm{c})},
$$

where $g$ is the quadrupole field gradient and $p$ is the momentum of the beam. The proportionality constant was defined as $(1 / B \rho)$ in section 3.1.

The sextupole magnets in the machine are modeled by the SEXT element. They are described by their length $L$ in meters and by their geometric sextupole strength $K 2$ in units of $1 / \mathrm{m}^{3}$ which is given by

$$
K 2=\frac{.29979 \times g^{\prime}\left(\mathrm{T} / \mathrm{m}^{2}\right)}{p(\mathrm{GeV} / \mathrm{c})},
$$

where $g^{\prime}$ is the sextupole field gradient and $p$ is the momentum of the beam.

Each of the dipole magnets in the machine are modeled by the CSBEND element. They are parameterized by their length $L$ in meters and the $A N G L E$ of the bend in radians. A quadrupole gradient term $K 1$, and a sextupole strength $K 2$ are used to capture the higher order components of the field. The entrance E1 and exit E2 angles of the beam relative to the pole face in radians are also defined and, along with the $H G A P$ parameter, determine the strength of the edge focusing for the dipole. 
The kicker magnets in the model are either oriented horizontally (HKICK) or vertically (VKICK) and are typically defined as zero length elements. The relevant parameter for driving the simulation is their $K I C K$ value which is given in radians. The BPMs in the model are represented by the MONITOR element and are also captured as zero length elements. They are located in the model to have the longitudinal center of the real BPM antennae at the design location. And finally the space between beamline components is referred to as a DRIFT which are simply defined by their length $L$ in meters.

The elegant file used to setup and run the simulations is shown in Appendix A.2. The first part is the run_setup section which specifies the lattice file and beamline for the simulation, defines the momentum of the beam, and the expected output files from the simulation. For this experiment the only required output is the beam centroid file which contains the beam position and angle at each element in the beamline for each step in the simulation.

The next segment is the run_control section which defines the number of times to run through the simulation defined by the following vary_element commands. For this simulation we simultaneously step each of the kicker magnets based on the value in the enumeration_file called corrector.sdds. The first column is composed of $1 \mathrm{~Hz}$ sinusoidal data while the next column has the $21 \mathrm{~Hz}$ sinusoidal data. The sinusoids were created using data from a real beam run as recorded in the data acquisition system.

Next in the setup file is the bunched beam section which defines the number of particles to track and some initial beam parameters. For these simulations the bunch is restricted to a single particle since detailed information about the beam and its evolution were not relevant to the study. Rather we are only interested in the point to point transport across the lattice. Finally the track section simply tells the software to record the tracked particle at each step in the simulation.

In the first simulation the sextupole at 1A14 was studied. The beam was modulated in the $y$-plane with the first kicker at $1 \mathrm{~Hz}$ and the second kicker at $21 \mathrm{~Hz}$. The modulation pattern was centered in the $x$-plane within the sextupole. Figure 51 shows the $x$ position and the FFT of the position with the sextupole set to $1000 \mathrm{G} / \mathrm{cm}$. The spectra clearly shows the harmonic sidebands $(2,20,22$, and $42 \mathrm{~Hz})$ of the two driving frequencies that one would expect for a field that depends quadratically on position. The simulation was repeated for multiple sextupole excitations to verify 

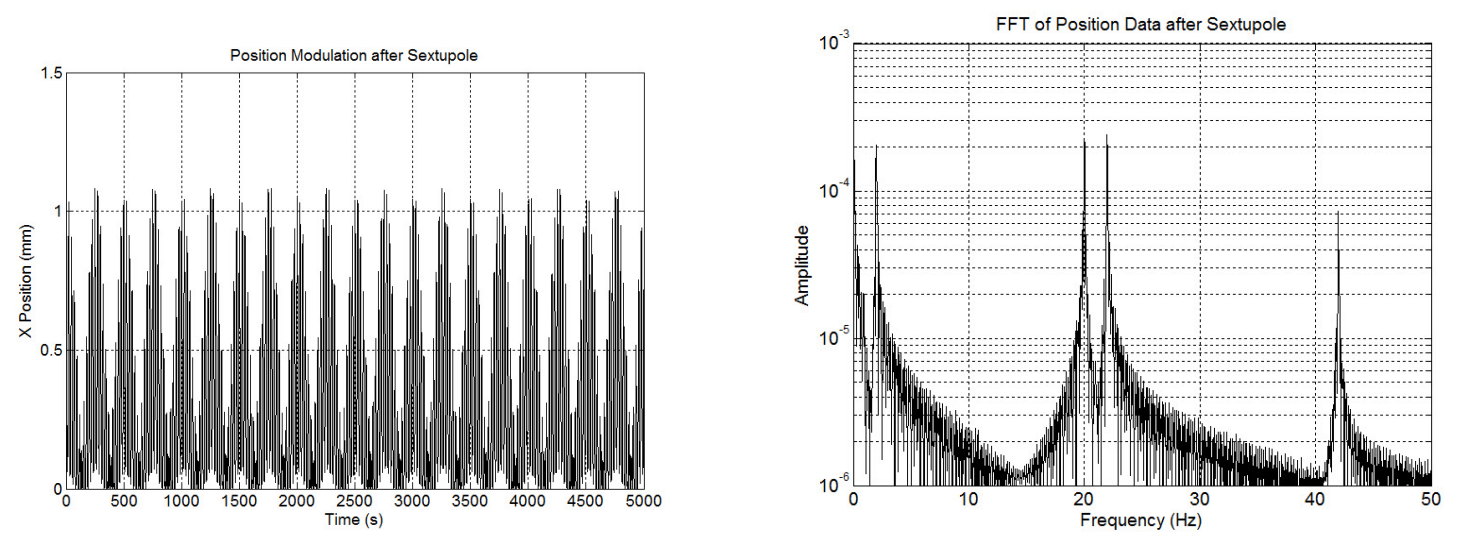

FIG. 51. Horizontal position and the FFT downstream of a sextupole at $1000 \mathrm{G} / \mathrm{cm}$.

the expected response from the analytical model developed in section 3.3. The results are shown at the top in Fig. 52 and clearly show the linear dependence of the sideband amplitudes with sextupole strength.

In the second simulation a sextupole field was added to a pair of ARC1 dipoles (MBE1A06 and MBE1A07) by adding a $K 2$ term to the model for each element. The simulation used the same modulation pattern as was used for the sextupole simulations. The intent of this study was to gauge the dependence of the multipole amplitudes on the horizontal position of the beam within a dipole. For the real machine study the orbit was horizontally shifted in a pair of dipoles using a four corrector orbit bump to provide a lateral position error within the magnets parallel to the normal trajectory. In the simulation the beam was also offset within both dipoles but rather than introduce an orbit bump we simply assigned an alignment error in the $x$-plane to shift the dipole pair about the design orbit. The results are shown at the bottom of Fig. 52 and are in qualitative agreement with the earlier TOSCA studies and the magnet measurement data which also show an increase in sideband amplitude as a function of transverse position of the modulation pattern within the dipole. 

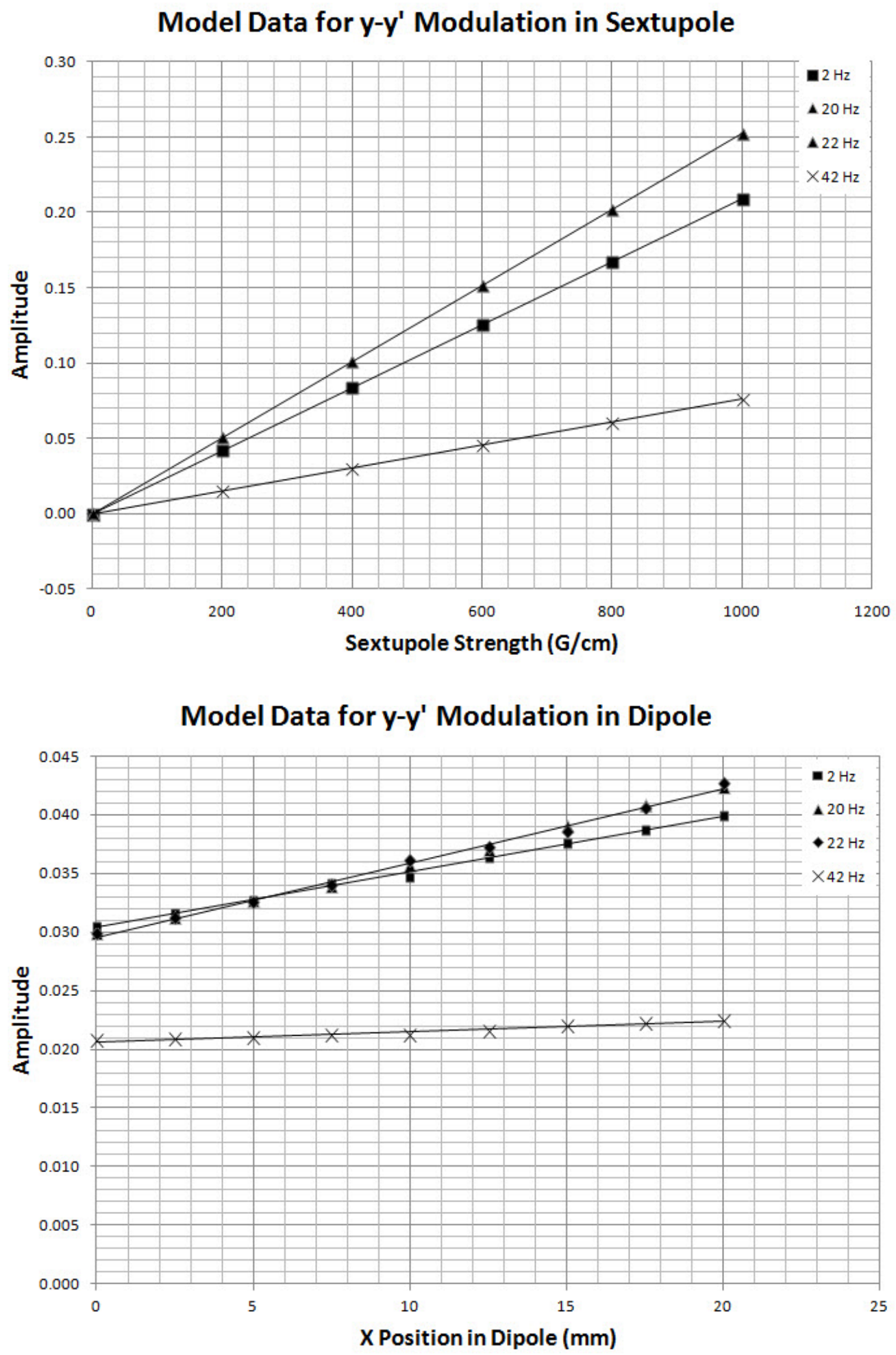

FIG. 52. Simulation results for the sextupole study and dipole study. 


\section{CHAPTER 6}

\section{BEAM MEASUREMENTS}

The beam-based measurements that were conducted for this thesis are presented in this chapter. In section 6.1 the overall experimental procedures for conducting the research are discussed. In section 6.2 a discussion of the NAFF algorithm that was used to find the amplitudes of the relevant frequencies in the spectra is presented. This is followed by a discussion of the systematic and random errors for the experiment in section 6.3. The first set of measurements involved beam modulations within an explicit sextupole magnet in the Arc 1 beamline and are presented in section 6.4. These measurements establish a calibration standard through the comparison of the amplitude of the sidebands of the modulation versus the strength of the sextupole. Once the calibration standard was developed the measurement technique was then applied to a pair of dipole magnets in Arc1 to determine the variation of their multipole strength versus the transverse position within the magnets. The Magnet Measurement Facility results of section 5.2, the TOSCA measurements of section 5.3, and the elegant simulations of section 5.4 all predict there to be a change in the spectra with position. The final set of measurements were conducted in the Arc 6 transport line to the Beam Switchyard. Here we gauge the overall field quality of the entire system of magnets that comprise a CEBAF recombiner beamline. The results are presented in section 6.6.

\subsection{EXPERIMENTAL PROCEDURE}

This section provides an overview of the steps taken to conduct the beam-based portion of this research. Prior to being awarded beam time on the accelerator a Test Plan must be submitted to the Operations Department for review. The plan outlines all of the necessary steps required to conduct the experiment and includes safety assessments as well as the identification of any prerequisites that need to be in place before the research can begin. Approved experiments are then scheduled well in advance which provides adequate time to familiarize the Operations staff with the details of the test plan. 
The prerequisite steps for this experiment were intended to qualify the hardware in the absence of beam. Prior to a beam run the data acquisition system is connected in the field to the BPM patch panel and kicker hardware as described in section 4.4. The BPM channels are calibrated without beam to determine the system's $\alpha$ values using the procedure described in section 4.2. The kicker magnets are then modulated to test their performance relative to the field maps described in section 4.1.

At the start of a beam test, the settings for the magnets in the accelerator are saved. These values are used to recover the accelerator after the tests are completed. The beam dump at the end of the beamline under test is inserted to ensure that the beam cannot be transported beyond the intended termination point (see Fig. 1). Low power tune beam, as discussed in section 4.3 is transported to the end of the line so that the beam steering can be optimized. The ideal trajectory for the electron beam is defined by the quadrupole centers along the lattice. This is checked by individually modulating each quadrupole field and monitoring the down beam transport. Since the field is zero at the center of a quadrupole, a well centered beam will not be deflected during quad modulation. An automated routine modulates the quadrupole setting while the beam is manually steered with corrector magnets. When the beam is in the center the downstream orbit deflections are at a minimum. Once this position is found an offset is entered into the adjacent BPM to match its electrical center with the magnetic center of the quad. This reference orbit can then be readily restored as necessary during the course of measurements.

Once the preliminary steps to establish a nominal orbit are completed the beam mode is changed to the $500 \mathrm{~Hz}, 100 \mu$ s structure required for the tests. The first beam measurement is typically performed without modulation to measure the inherent stability of the tune. An example of the stability of the beam centroid in the absence of explicit modulation is shown in Fig. 53. The dominant source of noise is due to AC line fluctuations on the magnet power supplies and is discussed further in section 6.3.

A typical sequence for beam measurements during sextupole runs is shown below.

- Verify nominal orbit with tune mode beam.

- Adjust sextupole setting and restore any orbit error due to minor steering errors within the sextupole.

- Switch to $500 \mathrm{~Hz}$ mode and turn on beam modulation with the pair of kickers. 


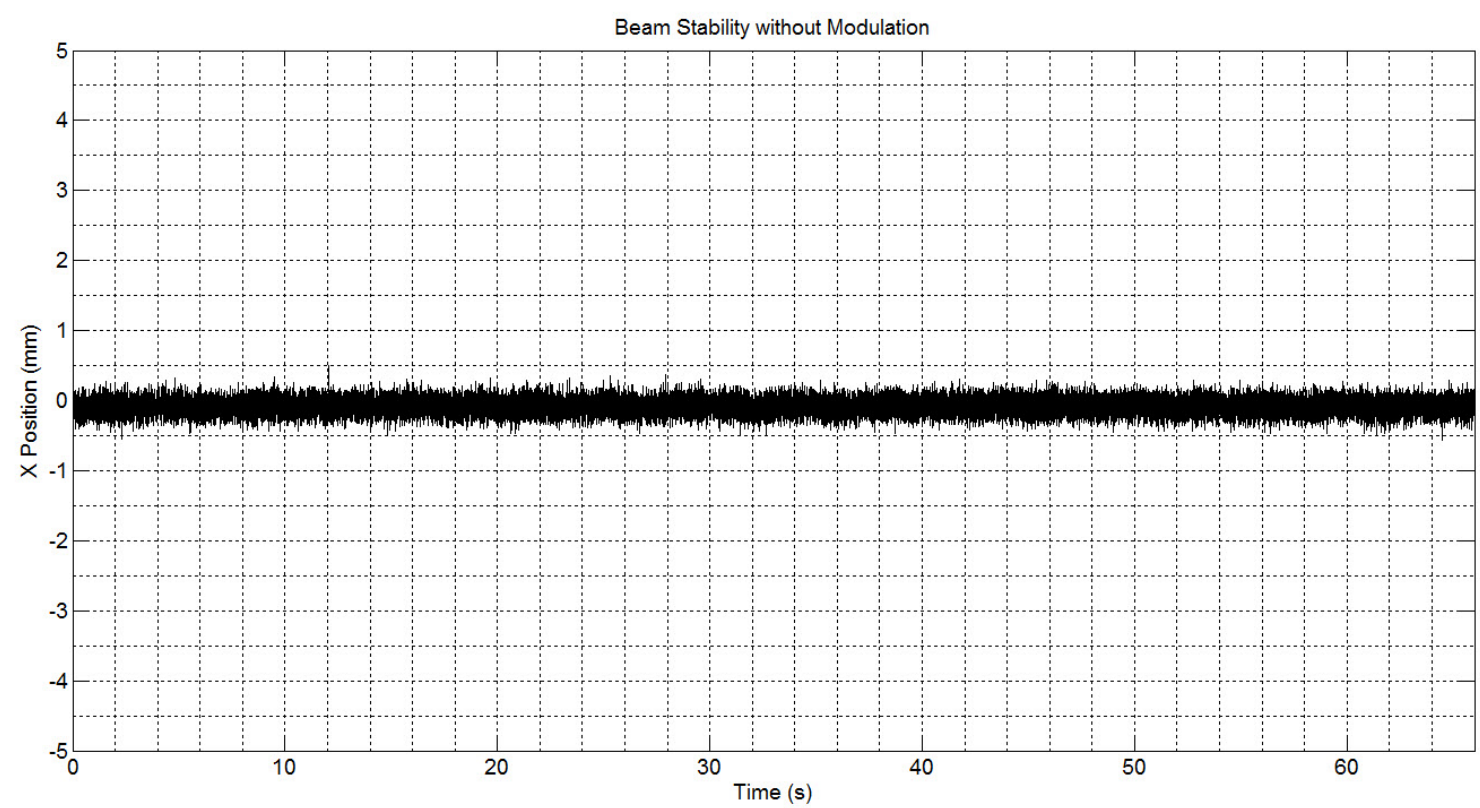

FIG. 53. Typical beam centroid stability showing the $x$ position as a function of time for Beam Position Monitor IPM1A16.

- Start the run acquiring 33000 samples in 66 seconds.

- Check online data quality at the end of the run to look for shifts in the average position that may come from uncontrolled errors in the transport.

- Turn off the beam modulation.

- Verify nominal orbit with tune mode beam.

- Adjust sextupole settings and or orbit for next measurement and repeat the sequence.

Once all of the runs were completed the modulation hardware and data acquisition system were shut down. The settings for the machine were restored and checked relative to the earlier save with tune mode beam. Dipole runs were performed in a similar way but instead of adjusting a sextupole excitation the beam orbit would be adjusted between measurements.

Off line analysis of the data was then performed and followed the sequence as outlined below and shown in the MATLAB code in Appendix B.

- Read BPM wire counts and kicker magnet voltages from data file. 
- Convert wire counts to $x$ and $y$ position data in the rotated frame.

- Correct for the nonlinearity of the calculated position using the Poisson model.

- Rotate the position data to the laboratory frame.

- Take Fast Fourier Transform of corrected position data.

- Find frequencies and amplitudes with NAFF algorithm.

\subsection{NAFF ALGORITHM}

The Numerical Application for Finding Frequencies was initially developed by Jacques Laskar [14] and was used in astrophysics research to determine the frequencies and amplitudes of orbits in complex galactic systems. This technique of frequency map analysis of Hamiltonian systems was recognized to be well suited to study the long term stability and dynamics of the quasi-periodic nonlinear orbits in particle accelerators [31].

The algorithm follows an iterative approach to find the complex frequencies and amplitudes and starts by first removing the average value of the time domain signal and applying a positive, even weighting function $\chi(t / T)$ to the standard definition of the FFT. We have

$$
\phi(\omega)=\left\langle f(t), e^{i \omega t}\right\rangle=\frac{1}{2 T} \int_{-T}^{T} f(t) e^{i \omega t} \chi(t / T) d t .
$$

To determine the first frequency one searches for the maximum amplitude using the above equation. For proper normalization the weighting function must satisfy

$$
1 / 2 \int_{-1}^{1} \chi(t) d t=1
$$

The NAFF algorithm used in this research employs a Hanning window as the weighting function given by

$$
\chi(t)=1+\cos (\pi t),
$$

which is readily found to satisfy Eq. (187). The Hanning window broadens the peaks while reducing the sidelobes which allows for a more precise determination of the frequencies and amplitudes. Once the maximum FFT amplitude is found the overlap is subtracted from the original signal and the process is repeated until the desired 
NAFF Amplitudes Relative to FFT

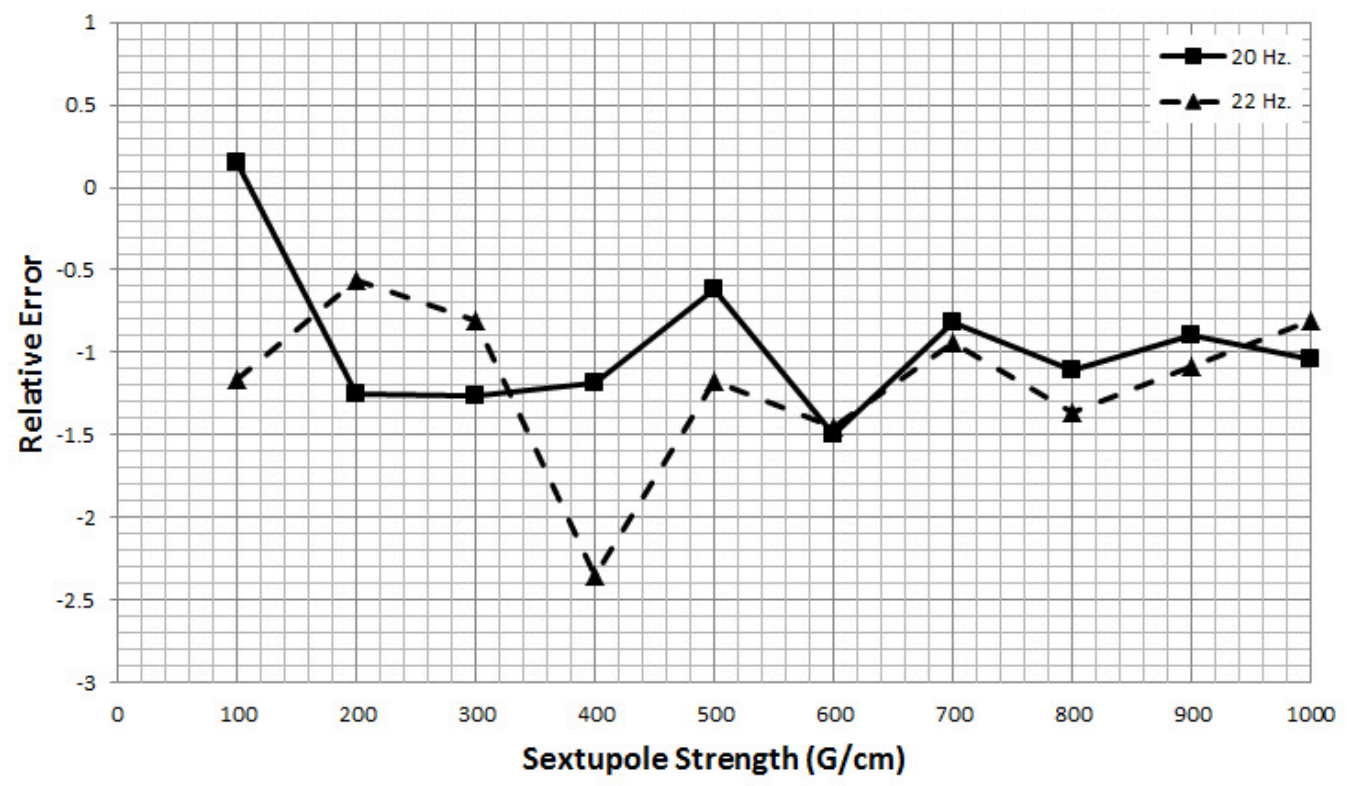

FIG. 54. A plot of the relative error in amplitude for FFT versus NAFF for the 20 and $22 \mathrm{~Hz}$ sidebands as a function of sextupole strength.

number of peaks is found. Figure 54 shows the comparison between NAFF and FFT with NAFF generally providing approximately a $1 \%$ correction to the sidebands at 20 and $22 \mathrm{~Hz}$ across the sextupole settings.

\subsection{MEASUREMENT ERROR}

As in all experiments there are systematic errors and random errors that contribute to the noise in the system and that can affect the quality of the data. The main source of systematic error is related to how well the beam is centered in the multipole. As was mentioned in section 6.1, a quadrupole modulation technique was used to find the magnetic center of the idealized beam trajectory. Keeping the beam on this same reference orbit throughout the measurement helps to minimize the systematic error. During a typical run the residual error at the downstream BPM was around 200 microns with full excitation of the sextupole to $1000 \mathrm{G} / \mathrm{cm}$. This is equivalent to having an orbit error relative to the ideal trajectory at the sextupole of around $1.4 \mathrm{~mm}$. This is a relatively large systematic error that could have been managed better. On the other hand one could also consider the sideband amplitude 
Flatness of $20 \mathrm{~Hz}$ Peak vs. Position

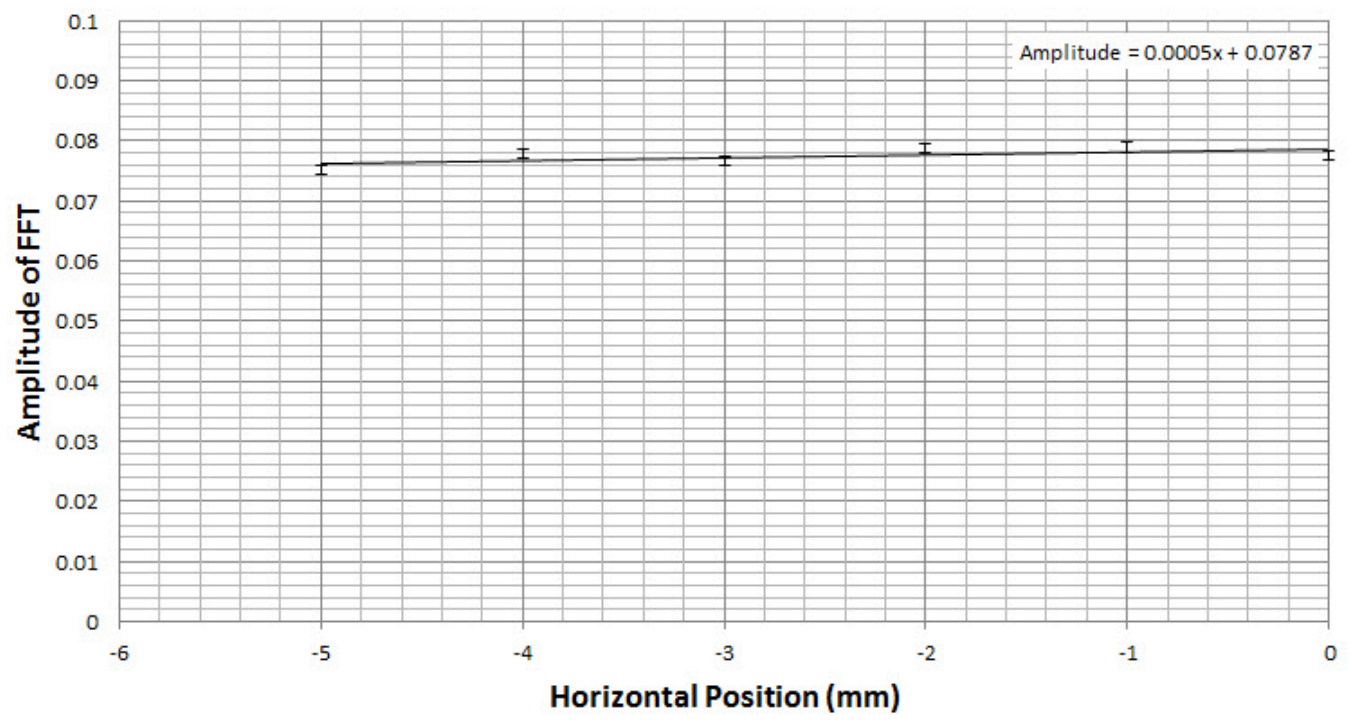

FIG. 55. A plot of the amplitude of the $20 \mathrm{~Hz}$ peak for $y-y^{\prime}$ modulation as a function of $x$ position within the sextupole.

sensitivity to transverse orbit error within the sextupole. By modulating the beam in the $y$-plane at different $x$ positions and recording the spectra an estimate of this sensitivity is determined. An example of the result for the $20 \mathrm{~Hz}$ sideband is shown in Fig. 55. The slope of the fit with $1 \%$ error bars is $5 \times 10^{-4}$. So we see that the amplitude is relatively insensitive to the position of the beam centroid within the sextupole.

The main source of statistical noise on the beam is due to the $60 \mathrm{~Hz}$ and higher harmonics that come from Arc magnet power supply fluctuations. These errors are in both planes with the vertical fluctuations coming from the magnets in the East Arc Spreader and the horizontal fluctuations coming from the Dogleg and East Arc magnets. The amplitude of these errors at each location depend on the transport optics between the many source points and the Beam Position Monitors. In Fig. 56 the noise at IPM1A16 is plotted as an example of the typical centroid stability with $\sigma_{x}=230 \mu \mathrm{m}$ and $\sigma_{y}=188 \mu \mathrm{m}$. The FFT of the position data in both planes is shown in Fig. 57 with the frequency axis set to highlight the strength of the primary line harmonic at $60 \mathrm{~Hz}$.

In a beam modulation experiment such as this, one can minimize the effect of 

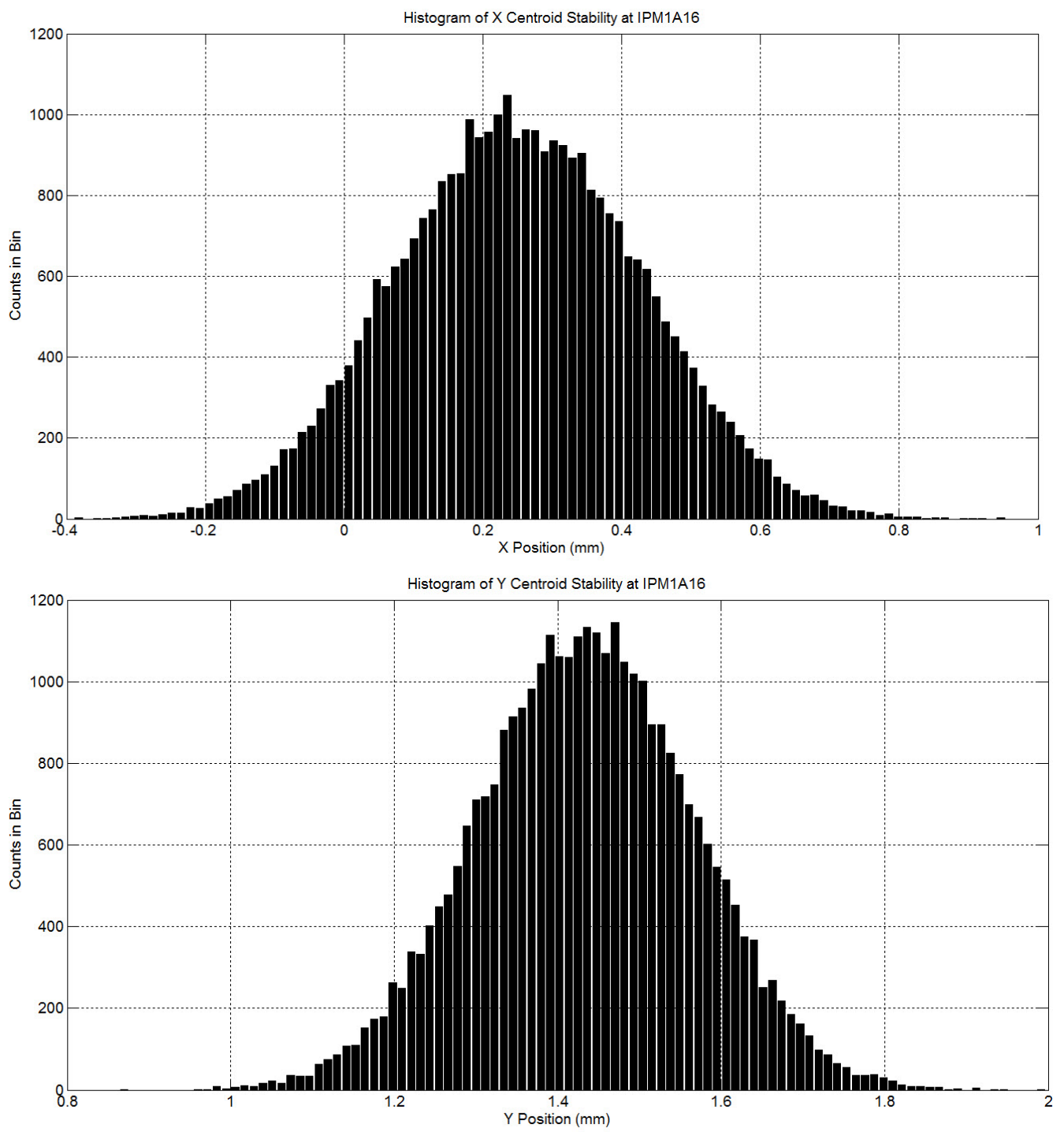

FIG. 56. Plot of $x$ and $y$ centroid noise at IPM1A16. The main source of peak broadening is due to $\mathrm{AC}$ ripple on magnet currents. 

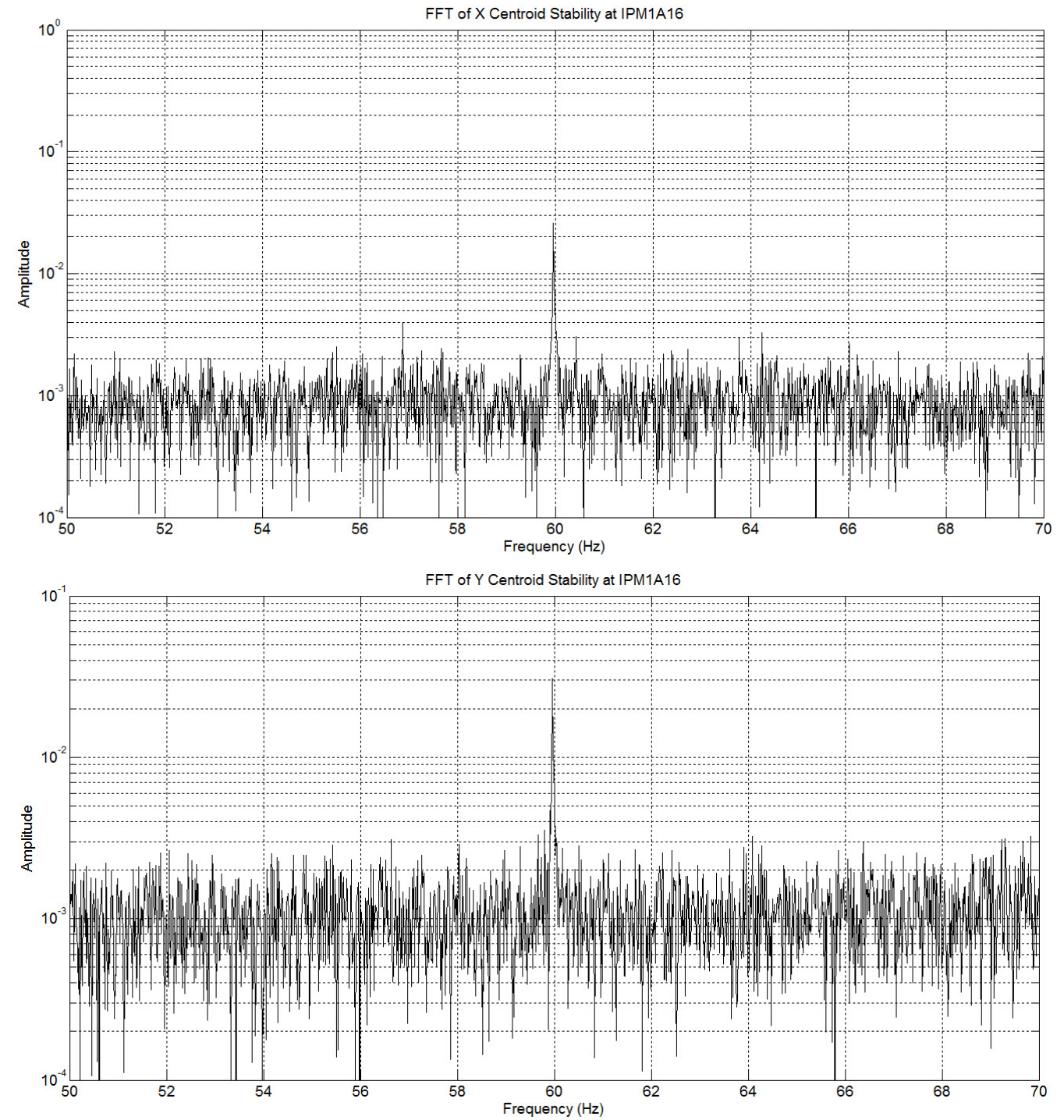

FIG. 57. FFT of $x$ and $y$ centroid noise at IPM1A16 showing the amplitude of the $60 \mathrm{~Hz}$ AC ripple on magnet currents. 


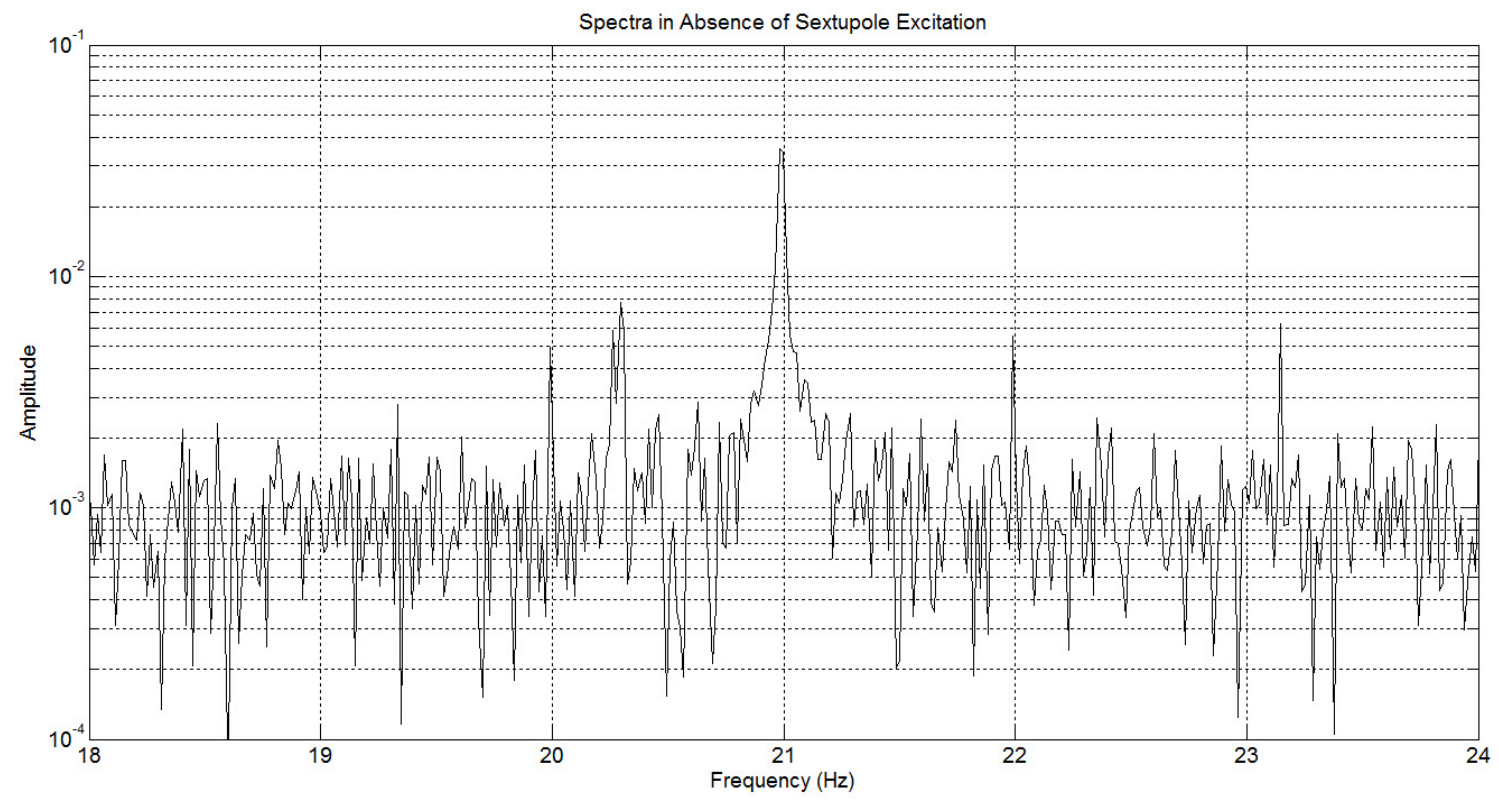

FIG. 58. The few parts in a thousand amplitude of the residual peaks in the absence of sextupole excitation sets a lower bound on the measurement error.

power supply noise by carefully selecting the kicker frequencies so that there is sufficient separation of the driving frequencies and their harmonics from the $\mathrm{AC}$ line peaks. Picking $1 \mathrm{~Hz}$ and $21 \mathrm{~Hz}$ works well for studying the beam transport system in the presence of the harmonics due to sextupole and octupole fields as shown in Table 3. The narrowness of the harmonic peaks also make it easy to distinguish the different frequencies in the spectra.

The dominant source of uncertainty for the sextupole calibration runs is related to the residual peaks in the spectra with the sextupole off. The plot in Fig. 58 shows that even in the absence of explicit sextupole excitation there are still measurable peaks that are discernable above the background. The beamline between the kicker magnets and the sextupole under test has many potential sources of nonlinearity. There are two half-meter long and a one-meter long dipole in the dogleg system as well as six one-meter long dipoles in the Arc proper that all potentially can contribute to the residual nonlinearity. For this experiment the limited number and location of Beam Position Monitors coupled with the long distance between the AC kickers and the magnets under test provide a source of error. The final statistical noise source for the experiment is due to the stochastic fluctuations in the system which set the overall noise floor and the limit for the best that one can do regarding the signal to 

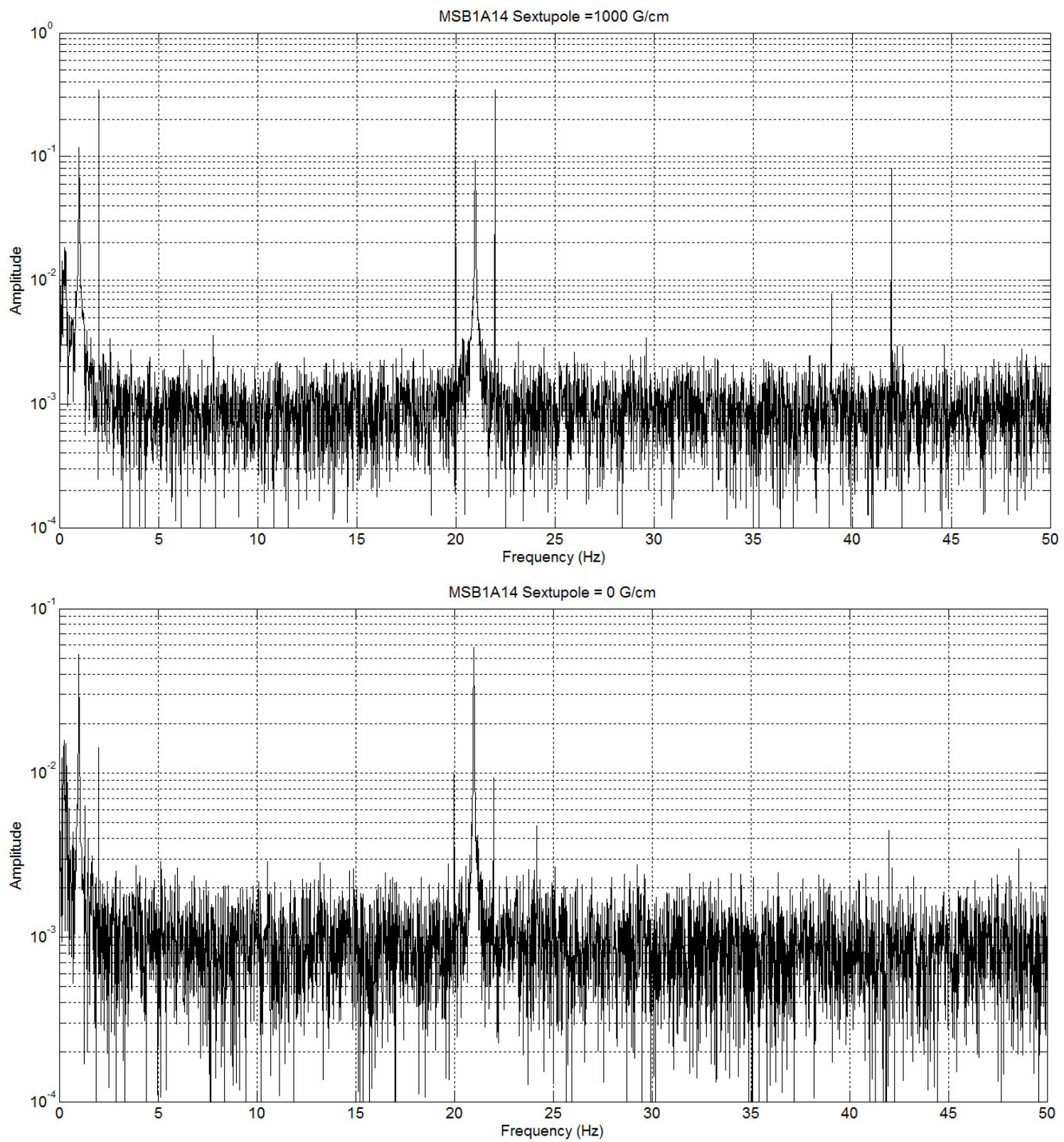

FIG. 59. Upper plot shows the spectra with the sextupole set to $1000 \mathrm{G} / \mathrm{cm}$. Lower plot with sextupole ramped to zero showing only the remnant field in the magnet. 
noise ratio. The plots in Fig. 59 show the spectra at the peak sextupole excitation as well as the case with the sextupole ramped to zero. The remnant field in the magnet is discernable above the background.

\subsection{SEXTUPOLE MEASUREMENTS}

In this section the results from the calibration runs are presented. The beam was modulated in the horizontal midplane and then in the vertical midplane for multiple settings on the sextupole. The beam centroid data for a typical run in the $y$-plane is shown in Fig. 60. Here we see that the modulations stay in plane until we cross the sextupole MSB1A14 which for this run was set to $1000 \mathrm{G} / \mathrm{cm}$. Beyond the sextupole starting at BPM $1 \mathrm{~A} 16$ we see the $y$-plane modulations become coupled to the $x$ plane due to the vertically oriented magnetic fields along the $y$ midplane. For the $x$-plane modulations of Fig. 61 we see no folding of the modulation pattern to the other plane. This is because the fields along the $x$ midplane are transverse to the modulation pattern and the Lorentz forces are in the plane of modulation.

For the calibration runs the field within the sextupole was varied from 0 to 1000 $\mathrm{G} / \mathrm{cm}$ in steps of $100 \mathrm{G} / \mathrm{cm}$. At each sextupole setting the beam orbit was restored to the nominal orbit with tune beam to correct for any minor steering error from the sextupole fields. This ensures that the beam modulation in the downstream BPM was always centered about the same point.

The analytical model of section 3.3 predicts a linear relationship for the amplitude of the harmonic frequencies with sextupole excitation. The data for the $y$-plane modulation is plotted in Fig. 62 and the data for the $x$-plane modulation runs is plotted in Fig. 63. For the $x$-plane modulations the sideband amplitudes below 400 $\mathrm{G} / \mathrm{cm}$ were overcome by the broad $1 \mathrm{~Hz}$ and $21 \mathrm{~Hz}$ primary modulation frequencies as they are all in the same plane. On the other hand the cross plane coupling under $y$-plane modulations allows for excellent separation of the harmonics from the driving frequencies as is shown in the bottom of the figure.

The data for the calibration runs is shown in Tables 11 and 10 for the $x$-plane and $y$-plane respectively. An entry of NA means that there was no discernable peak at that frequency and sextupole setting.

Both sets of measurements show a linear dependence of sideband amplitude with sextupole excitation. The lack of signal at small sextupole settings under horizontal excitation makes it difficult to measure the relatively weak signal from dipoles as was 

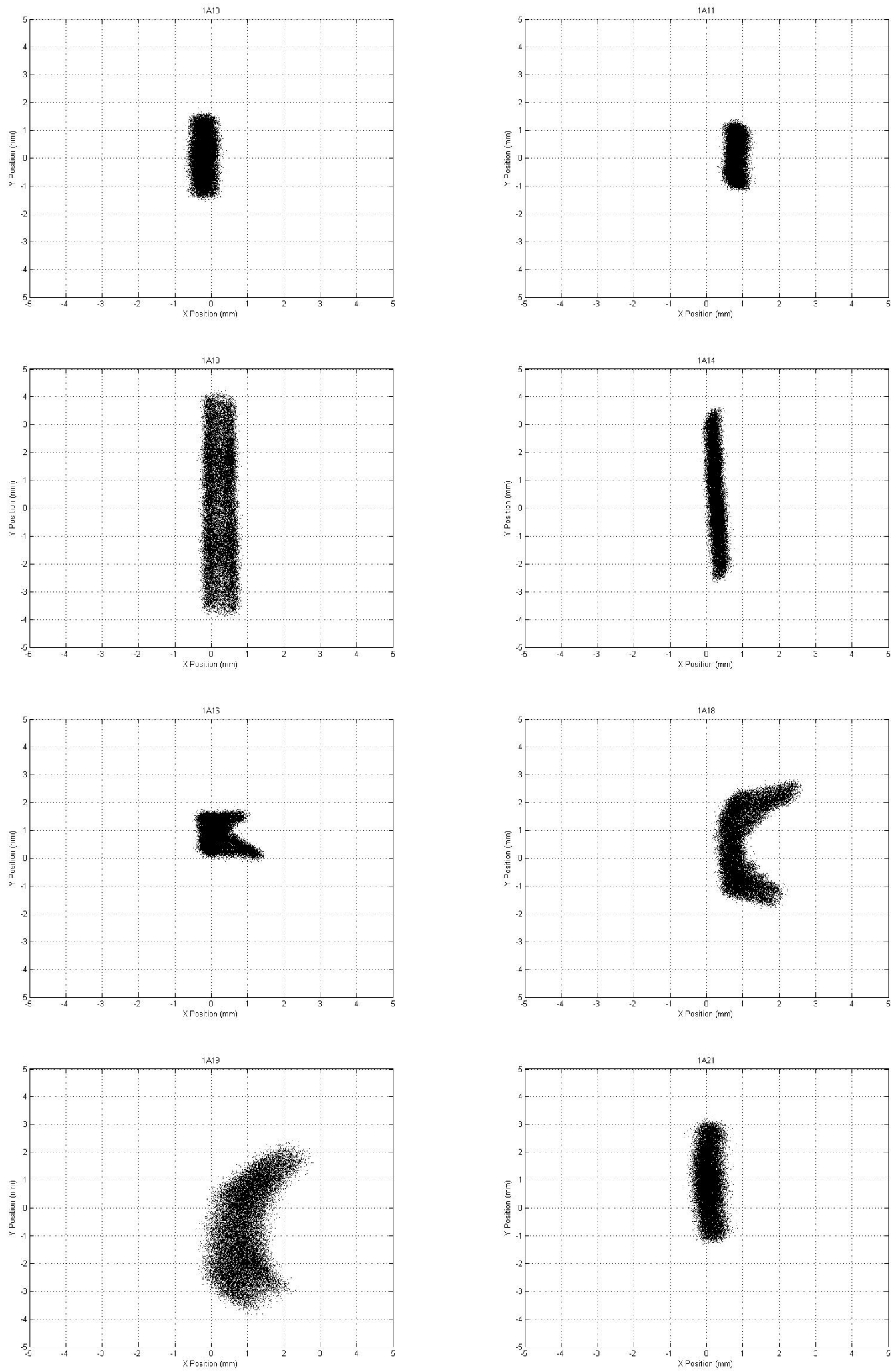

FIG. 60. Plots of the transverse position for all eight BPMs during a sextupole run with $y-y^{\prime}$ modulation. Cross plane coupling is evident starting at 1A16. 

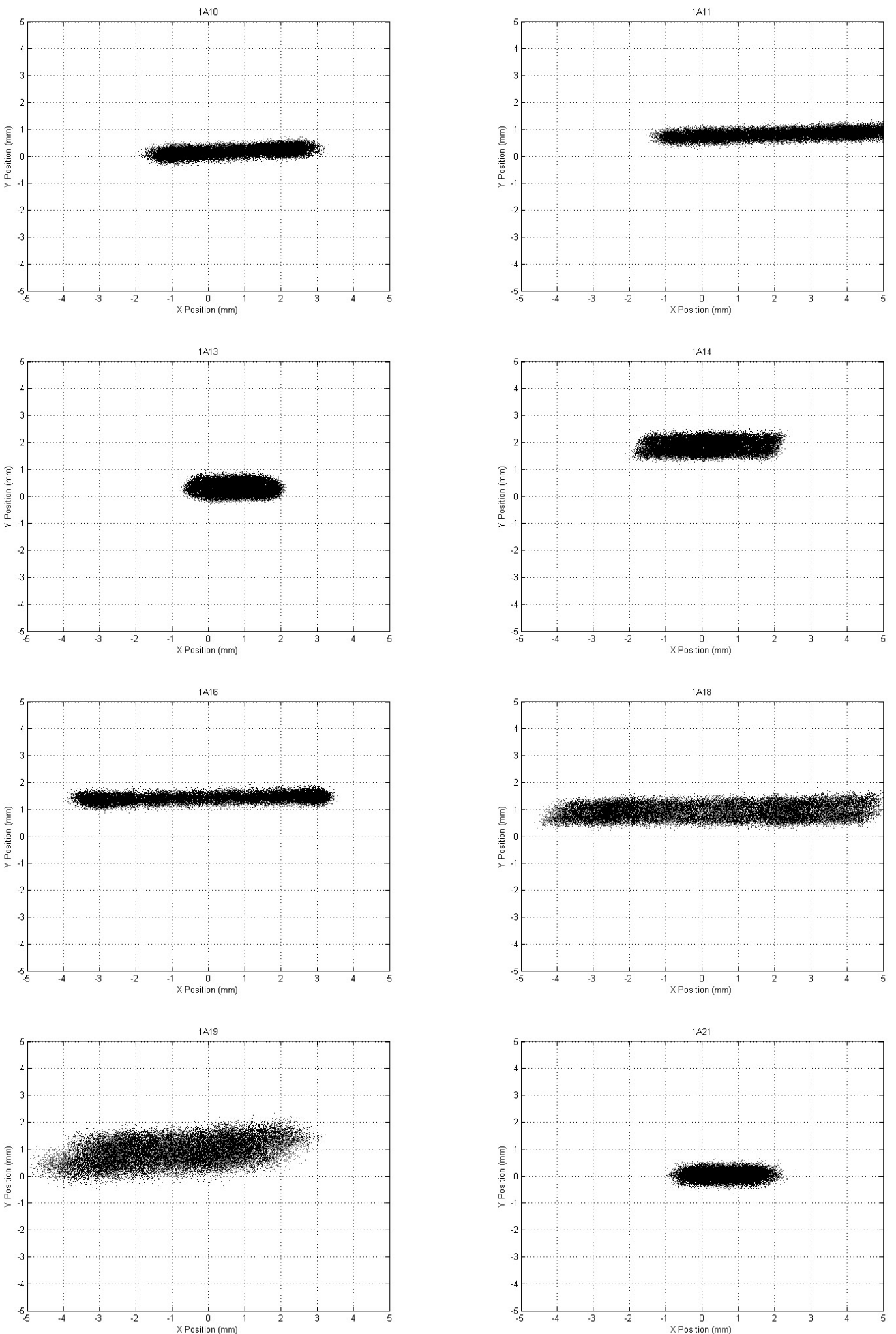

FIG. 61. Plots of the transverse position for all eight BPMs during a sextupole run with $x-x^{\prime}$ modulation. No cross plane coupling occurs. 
TABLE 10. Spectral data for $y-y^{\prime}$ modulation in Sextupole mid-plane

\begin{tabular}{ccccccc}
\hline \hline Sextupole $(\mathrm{G} / \mathrm{cm})$ & $1 \mathrm{~Hz}$ & $2 \mathrm{~Hz}$ & $20 \mathrm{~Hz}$ & $21 \mathrm{~Hz}$ & $22 \mathrm{~Hz}$ & $42 \mathrm{~Hz}$ \\
\hline 0 & 0.008 & 0.0139 & $\mathrm{NA}$ & 0.0092 & $\mathrm{NA}$ & $\mathrm{NA}$ \\
100 & 0.1002 & 0.0481 & 0.0451 & 0.0994 & 0.0442 & NA \\
200 & 0.1111 & 0.0782 & 0.0768 & 0.1052 & 0.0782 & 0.0175 \\
300 & 0.1228 & 0.1134 & 0.1094 & 0.1123 & 0.1104 & 0.0209 \\
400 & 0.1293 & 0.1435 & 0.1424 & 0.1183 & 0.1409 & 0.0295 \\
500 & 0.1384 & 0.1762 & 0.1768 & 0.1189 & 0.1766 & 0.0371 \\
600 & 0.1547 & 0.2101 & 0.2105 & 0.1281 & 0.2113 & 0.0467 \\
700 & 0.1569 & 0.2429 & 0.2439 & 0.1312 & 0.2438 & 0.0562 \\
800 & 0.1704 & 0.2769 & 0.2783 & 0.1365 & 0.2755 & 0.0635 \\
900 & 0.1723 & 0.3074 & 0.3101 & 0.1408 & 0.3100 & 0.0698 \\
1000 & 0.1826 & 0.3399 & 0.3428 & 0.1437 & 0.3437 & 0.0790 \\
\hline \hline
\end{tabular}

TABLE 11. Spectral data for $x-x^{\prime}$ modulation in Sextupole mid-plane

\begin{tabular}{ccccccc}
\hline \hline Sextupole $(\mathrm{G} / \mathrm{cm})$ & $1 \mathrm{~Hz}$ & $2 \mathrm{~Hz}$ & $20 \mathrm{~Hz}$ & $21 \mathrm{~Hz}$ & $22 \mathrm{~Hz}$ & $42 \mathrm{~Hz}$ \\
\hline 0 & 3.1527 & $\mathrm{NA}$ & 0.0447 & $\mathrm{NA}$ & $\mathrm{NA}$ & NA \\
100 & 3.1569 & $\mathrm{NA}$ & 0.0449 & $\mathrm{NA}$ & $\mathrm{NA}$ & NA \\
200 & 3.1573 & $\mathrm{NA}$ & 0.0488 & $\mathrm{NA}$ & $\mathrm{NA}$ & NA \\
300 & 3.1604 & 0.0159 & 0.0518 & $\mathrm{NA}$ & $\mathrm{NA}$ & $\mathrm{NA}$ \\
400 & 3.1597 & $\mathrm{NA}$ & 0.0225 & 0.0550 & 0.0214 & 0.0232 \\
500 & 3.1677 & 0.0207 & 0.0281 & 0.0577 & 0.0265 & 0.0257 \\
600 & 3.1656 & 0.0242 & 0.0361 & 0.0618 & 0.0366 & 0.0299 \\
700 & 3.1685 & 0.0273 & 0.0429 & 0.0648 & 0.0428 & 0.0348 \\
800 & 3.1715 & 0.0282 & 0.0499 & 0.0671 & 0.0471 & 0.0410 \\
900 & 3.1827 & 0.0304 & 0.0564 & 0.0808 & 0.0566 & 0.0445 \\
1000 & 3.1851 & 0.0358 & 0.0641 & 0.0861 & 0.0628 & 0.0488 \\
\hline \hline
\end{tabular}


Beam Data for y-y' Modulation in Sextupole

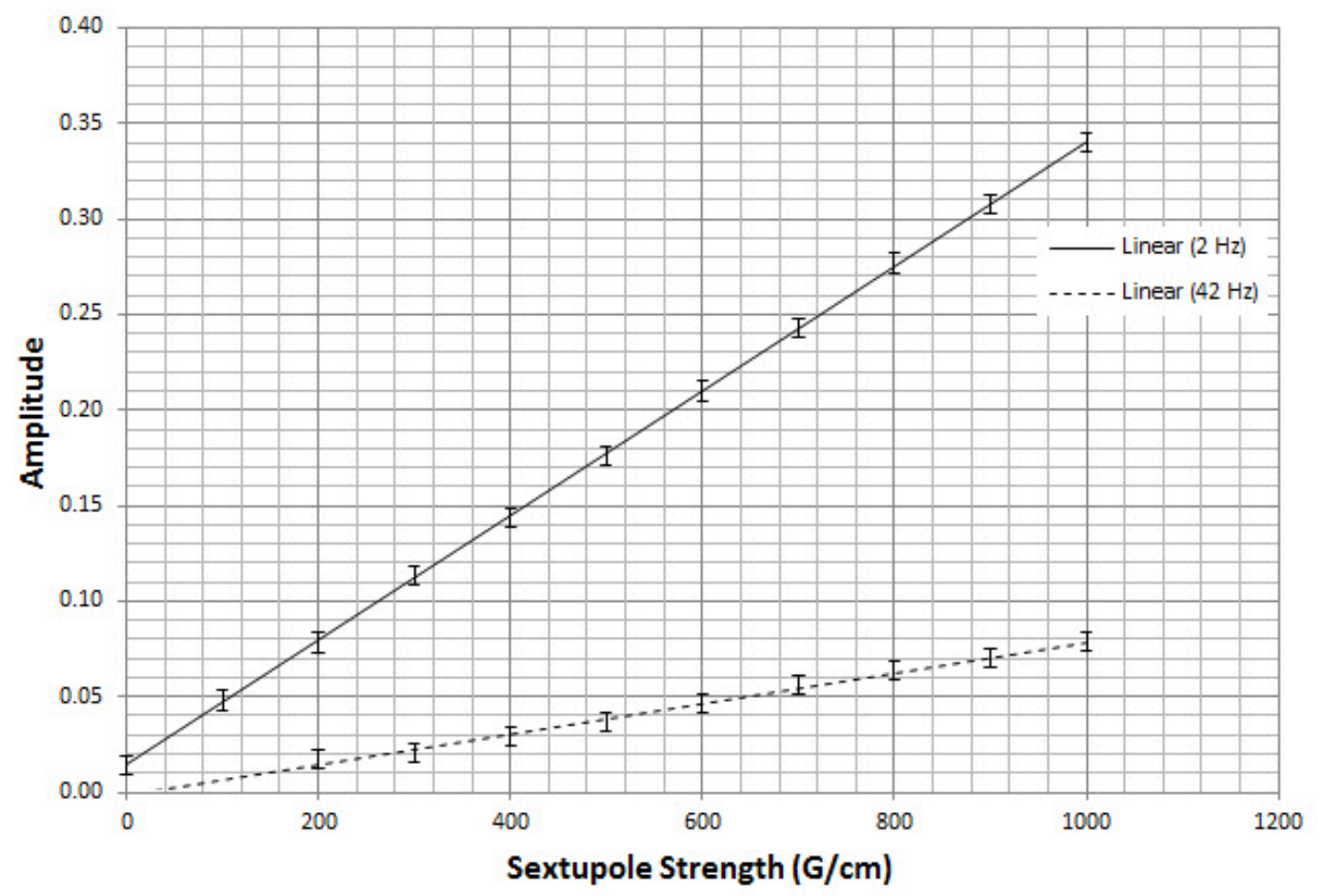

\section{Beam Data for y-y' Modulation in Sextupole}

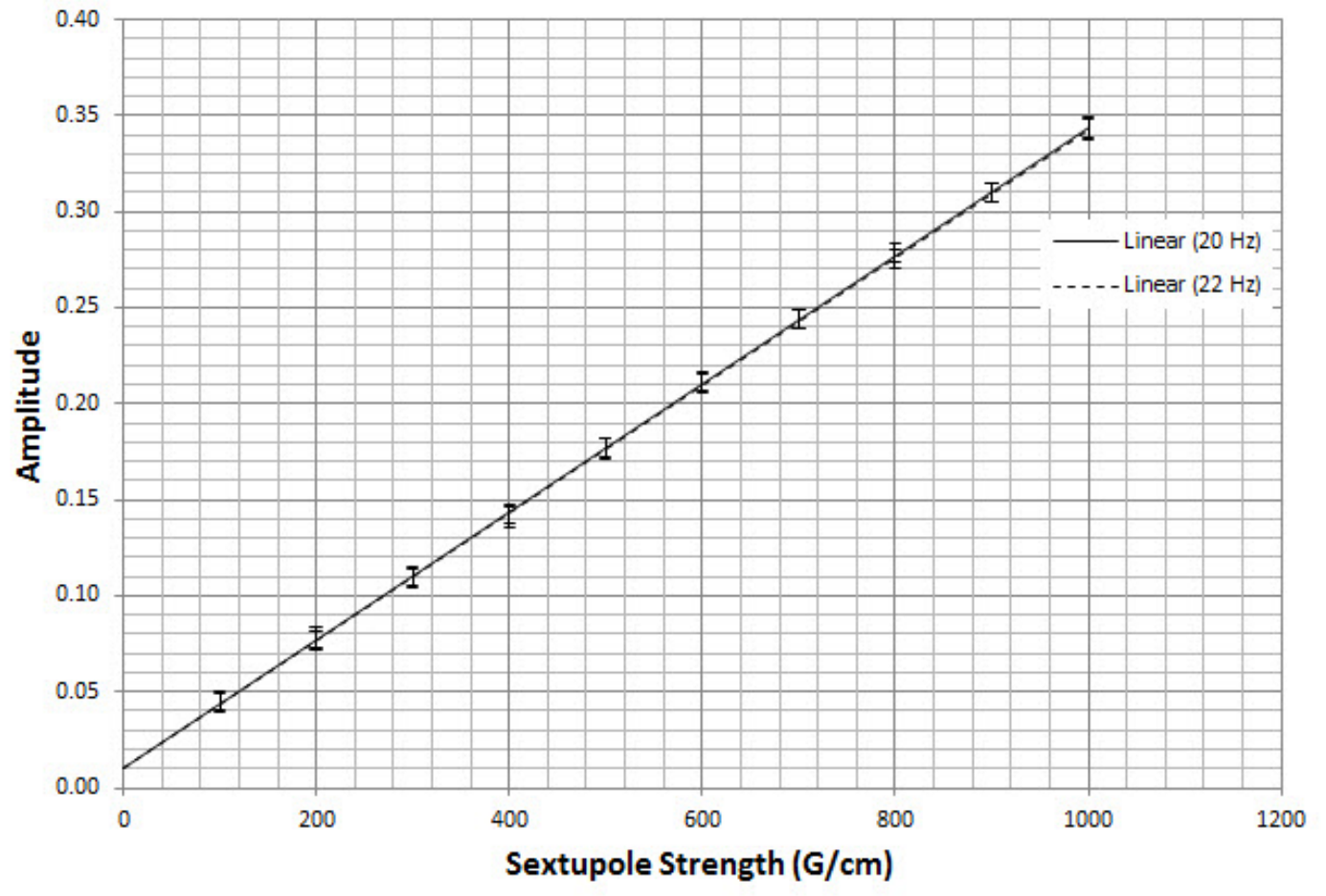

FIG. 62. Beam data for the sextupole measurements with $y$-plane modulation. The $2 \mathrm{~Hz}$ and $42 \mathrm{~Hz}$ data are at the top and the $20 \mathrm{~Hz}$ and $22 \mathrm{~Hz}$ data are at the bottom. 
Beam Data for $\mathbf{x}-\mathbf{x}^{\prime}$ Modulation in Sextupole
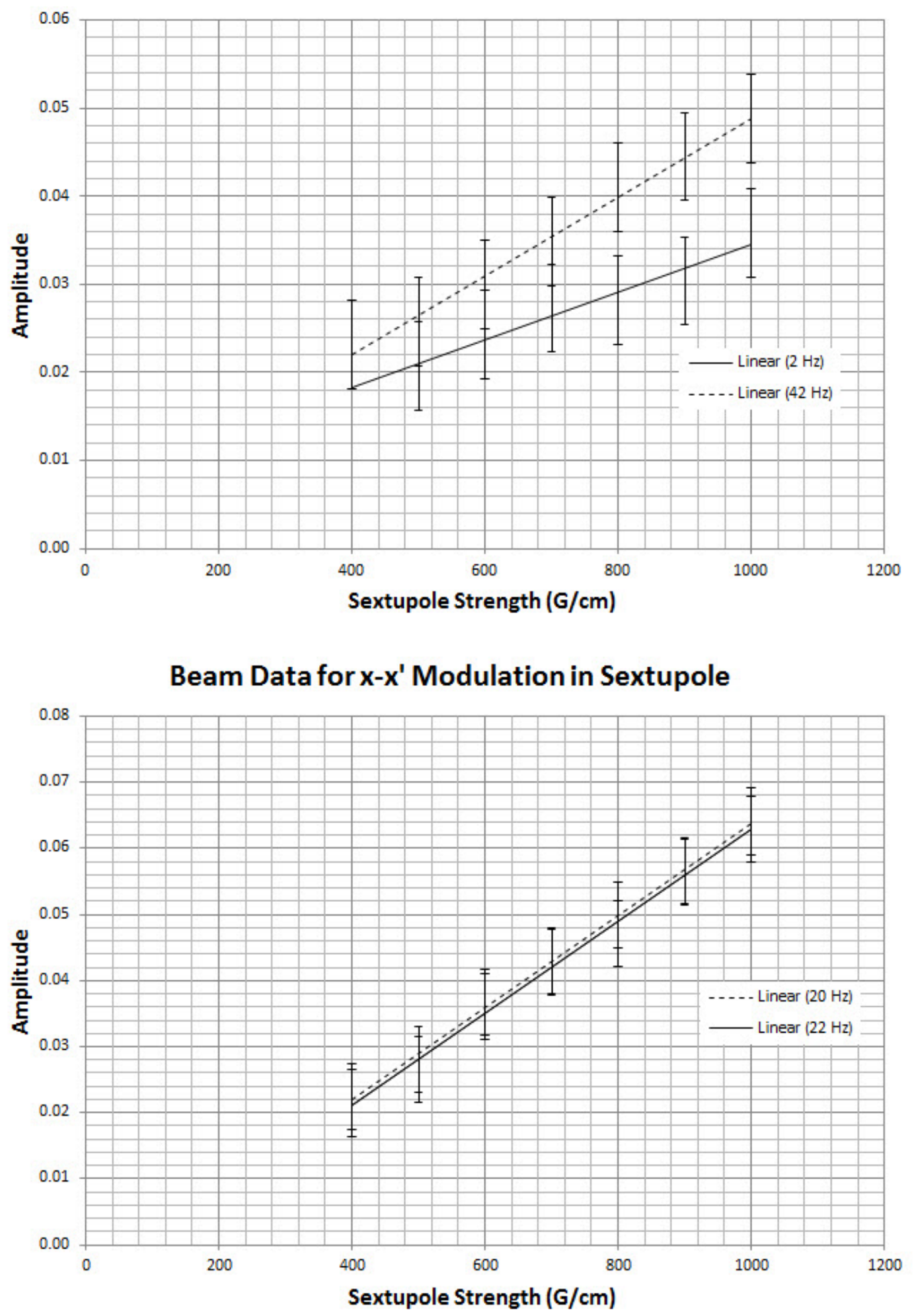

FIG. 63. Beam data for the sextupole measurements with $x$-plane modulation. The $2 \mathrm{~Hz}$ and $42 \mathrm{~Hz}$ data are at the top and the $20 \mathrm{~Hz}$ and $22 \mathrm{~Hz}$ data are at the bottom. 
found during the next phase of the beam measurements.

\subsection{BE DIPOLE MEASUREMENTS}

In this section the results for the dipole measurements in Arc 1 are presented. For this test the sextupole magnet used earlier was degaussed so that it would not interfere with measuring the relatively weak allowed multipole content of the dipole pair. As was discussed in section 5.4, a four corrector bump was used to offset the beam within the dipole magnets. The quadrupoles within the orbit bump were also degaussed to eliminate the quad kicks which would otherwise oppose the corrector kicks. For each measurement tune beam was used to establish a precise orbit across the dipole pair and to return the beam to the center of the BPM used to make the measurements.

The TOSCA measurements and magnet measurement data both show that the extent of the nonlinearity due to allowed sextupole should increase with an offset in the horizontal direction. The TOSCA results also showed a decrease in the multipole content as the beam rises above the midplane. Measurements of the dipole pair were done in both planes. For the horizontal tests the beam was modulated in the midplane and in $1 \mathrm{~mm}$ steps above the midplane to $5 \mathrm{~mm}$. There were no frequencies detected other than the primary driving frequencies and the $\mathrm{AC}$ line harmonics. This limitation was also observed during the sextupole calibration runs. During $x$ plane modulation the Lorentz forces are in the same plane as the beam modulation preventing the relatively weak signal from being detected. For the earlier sextupole calibration run there were no peaks below $400 \mathrm{G} / \mathrm{cm}$. Moving to the $y$-plane however offered better results. For these tests the beam was modulated in the $y$-plane at $x=0 \mathrm{~mm}$ to $x=10 \mathrm{~mm}$ in $1 \mathrm{~mm}$ steps.

A plot of the results for the dipole measurements is shown in Fig. 64. The raw data for the measurement is shown in Table 12 with the $x$ position corrected for the nonlinearity of the Beam Position Monitor. The linear trend is clear from the data for all frequencies and qualitatively compares to the results from TOSCA and the Magnet Measurement Facility. TOSCA also predicts that beyond a horizontal orbit of $12 \mathrm{~mm}$ the field begins to rise more sharply. The beam-based test are unfortunately limited by the physical aperture of the beam pipe. Attempting to modulate the beam beyond $1 \mathrm{~cm}$ caused beam scraping. The final point at $x=10.342 \mathrm{~mm}$ hints at this departure from the linear trend. 
Beam Data for $y-y$ ' Modulation in Dipole

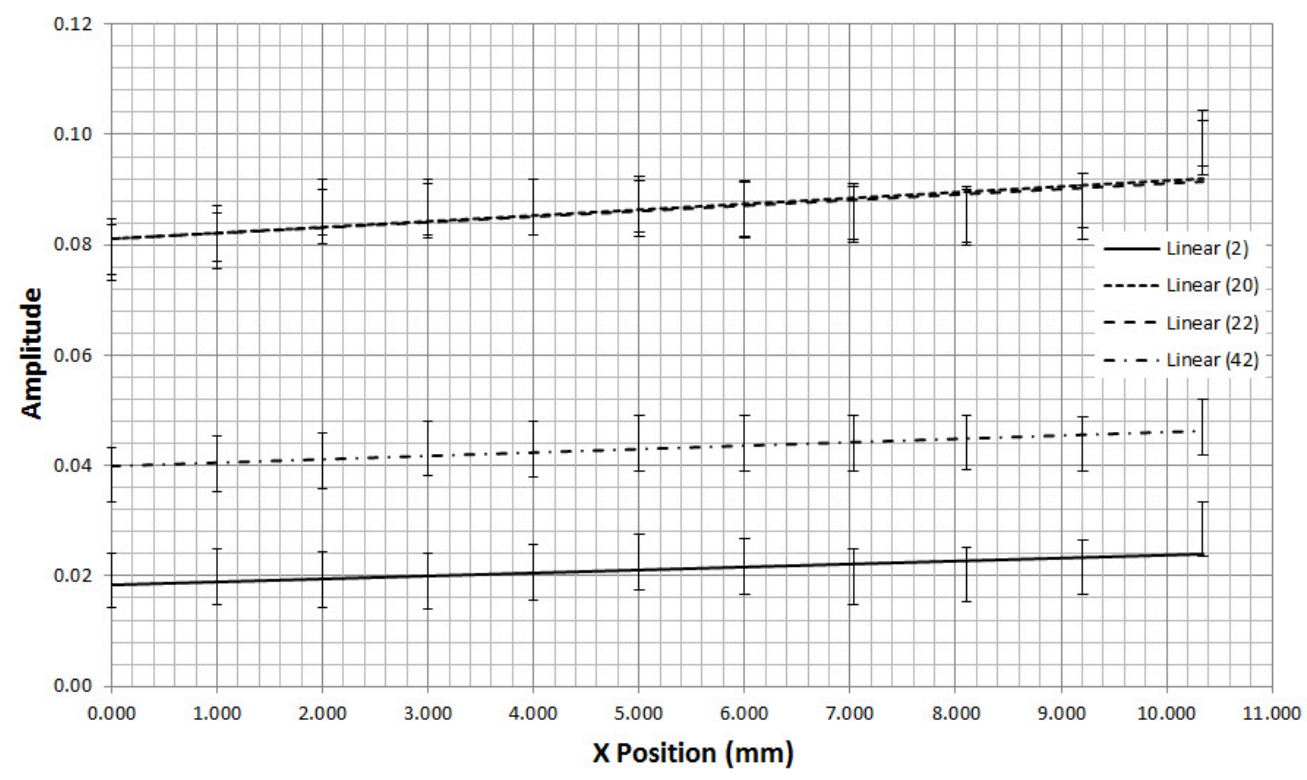

FIG. 64. Beam data for $y$-plane modulation in a pair of BE dipoles.

TABLE 12. Spectral data for $y-y^{\prime}$ modulation vs. $x$ position in BE Dipole

\begin{tabular}{ccccccc}
\hline \hline Corr. $x$-pos $(\mathrm{mm})$ & $1 \mathrm{~Hz}$ & $2 \mathrm{~Hz}$ & $20 \mathrm{~Hz}$ & $21 \mathrm{~Hz}$ & $22 \mathrm{~Hz}$ & $42 \mathrm{~Hz}$ \\
\hline 0.000 & 0.0391 & 0.0192 & 0.0786 & 0.0579 & 0.0797 & 0.0383 \\
1.000 & 0.0475 & 0.0199 & 0.0821 & 0.0776 & 0.0808 & 0.0403 \\
2.000 & 0.0488 & 0.0193 & 0.0851 & 0.0794 & 0.0869 & 0.0408 \\
3.000 & 0.0495 & 0.0191 & 0.0868 & 0.0789 & 0.0862 & 0.0431 \\
4.000 & 0.0482 & 0.0207 & 0.0869 & 0.0781 & 0.0869 & 0.0430 \\
5.000 & 0.0513 & 0.0224 & 0.0874 & 0.0766 & 0.0866 & 0.0440 \\
6.000 & 0.0514 & 0.0217 & 0.0866 & 0.0782 & 0.0864 & 0.0440 \\
7.033 & 0.0511 & 0.0198 & 0.0860 & 0.0761 & 0.0856 & 0.0441 \\
8.105 & 0.0480 & 0.0203 & 0.0855 & 0.0749 & 0.0850 & 0.0442 \\
9.205 & 0.0490 & 0.0216 & 0.0881 & 0.0737 & 0.0860 & 0.0439 \\
10.342 & 0.0423 & 0.0285 & 0.0976 & 0.0658 & 0.0993 & 0.0469 \\
\hline \hline
\end{tabular}




\subsection{BSY RECOMBINER MEASUREMENTS}

The final set of beam-based tests were conducted in the Beam Switchyard Recombiner using the MAZ kicker magnets that were fabricated as part of this research. The intent of these tests was to qualify the extent of the nonlinearity of the whole system of magnets by performing beam modulations in both planes at multiple amplitudes. To simplify the lattice the quadrupoles downstream of the kicker magnets were degaussed. Without the quad kicks it was much easier to establish the desired modulation amplitudes. For these test we chose amplitudes of $5 \mathrm{~mm}, 10 \mathrm{~mm}$ and, 15 $\mathrm{mm}$ at the BPM near the end of the beamline. The three different modulations in both plane are shown in Fig. 65.

The source of any nonlinearity in this transport line can come from errors in individual magnets or from the magnets in the adjacent beamlines which are tightly nested as the beamlines come together in the Beam Switchyard. A TOSCA model of this system had not been developed to compare against so we rely on the calibration runs and ARC1 dipole measurements to bound the field quality of the system. A linear result to $\pm 5 \mathrm{~mm}$ is adequate as that is typically the steering allowance used in setting up the machine. The results are shown in the FFT plots in Figs. 66 and 67.

The first set are the $x$-plane modulations. At $5 \mathrm{~mm}$ only the primary frequencies are visible in the modulation plane. Other than noise in the $y$-plane there's also a $1 \mathrm{~Hz}$ peak two orders of magnitude down from that in the $x$-plane which likely is due to a small roll error in the placement of the first horizontal kicker. At 10 $\mathrm{mm}$ we see the $1 \mathrm{~Hz}$ peak grow in the $y$ plane and in the $x$-plane we start to see frequencies consistent with an octupole field which are even more pronounced in the bottom plot. At these amplitudes we begin to approach the limit of the polynomial correction for the BPM nonlinearity which was only computed to a $2 \mathrm{~cm}$ grid or an apparent position of around $16 \mathrm{~mm}$.

The second set of measurements are with $y$-plane modulations. At $5 \mathrm{~mm}$ we see the strong driving frequencies at the right and coupling to the other plane at both frequencies. The roll error for both vertical kickers is apparently over three degrees and will need to be checked at the next opportunity. At $10 \mathrm{~mm}$ we again start to see octupole sidebands which become more apparent in the bottom trace and once again likely due to limitations with the correction algorithm. 

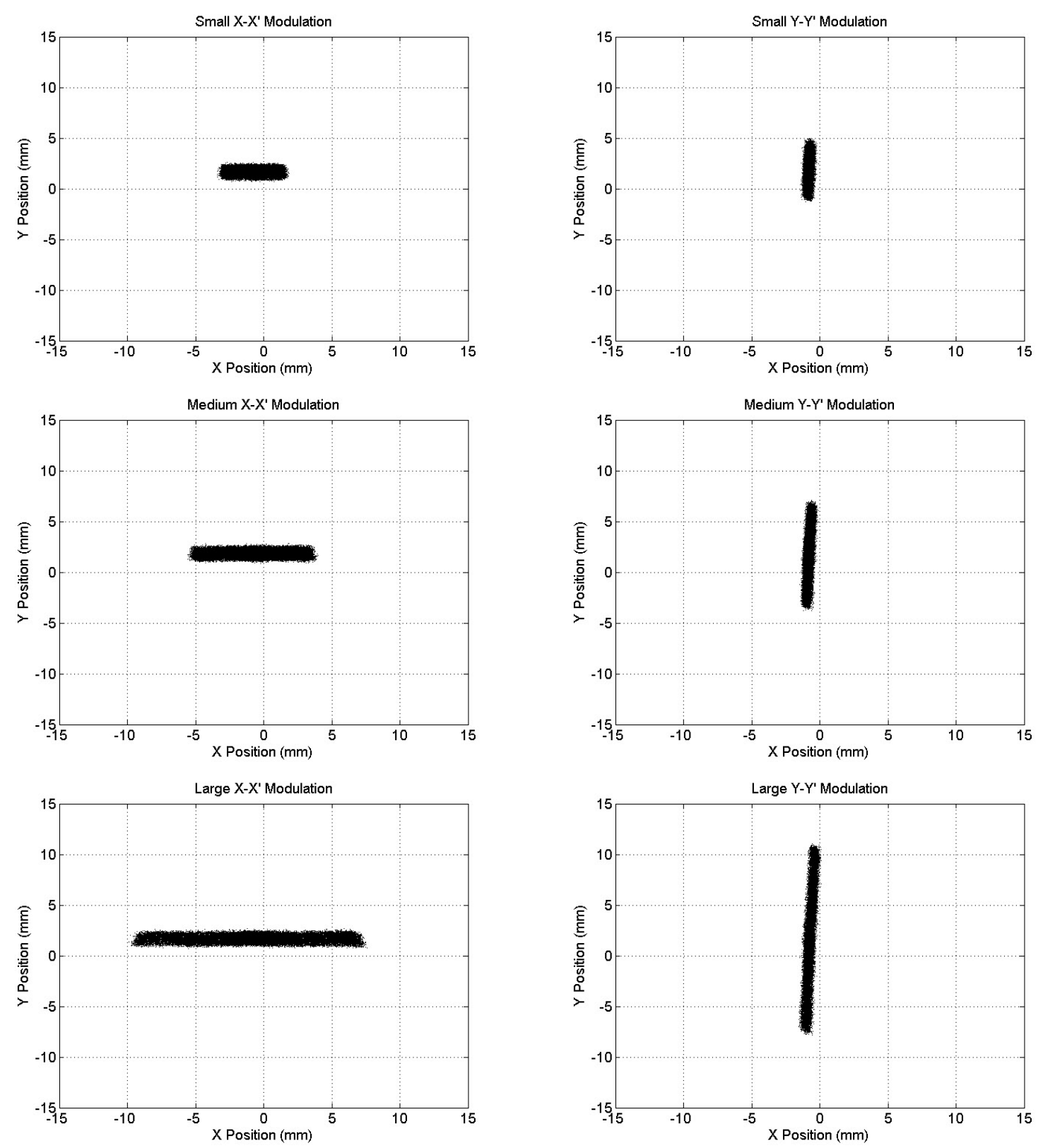

FIG. 65. Plots of small, medium and large beam modulations as measured at the 6T09 BPM. 

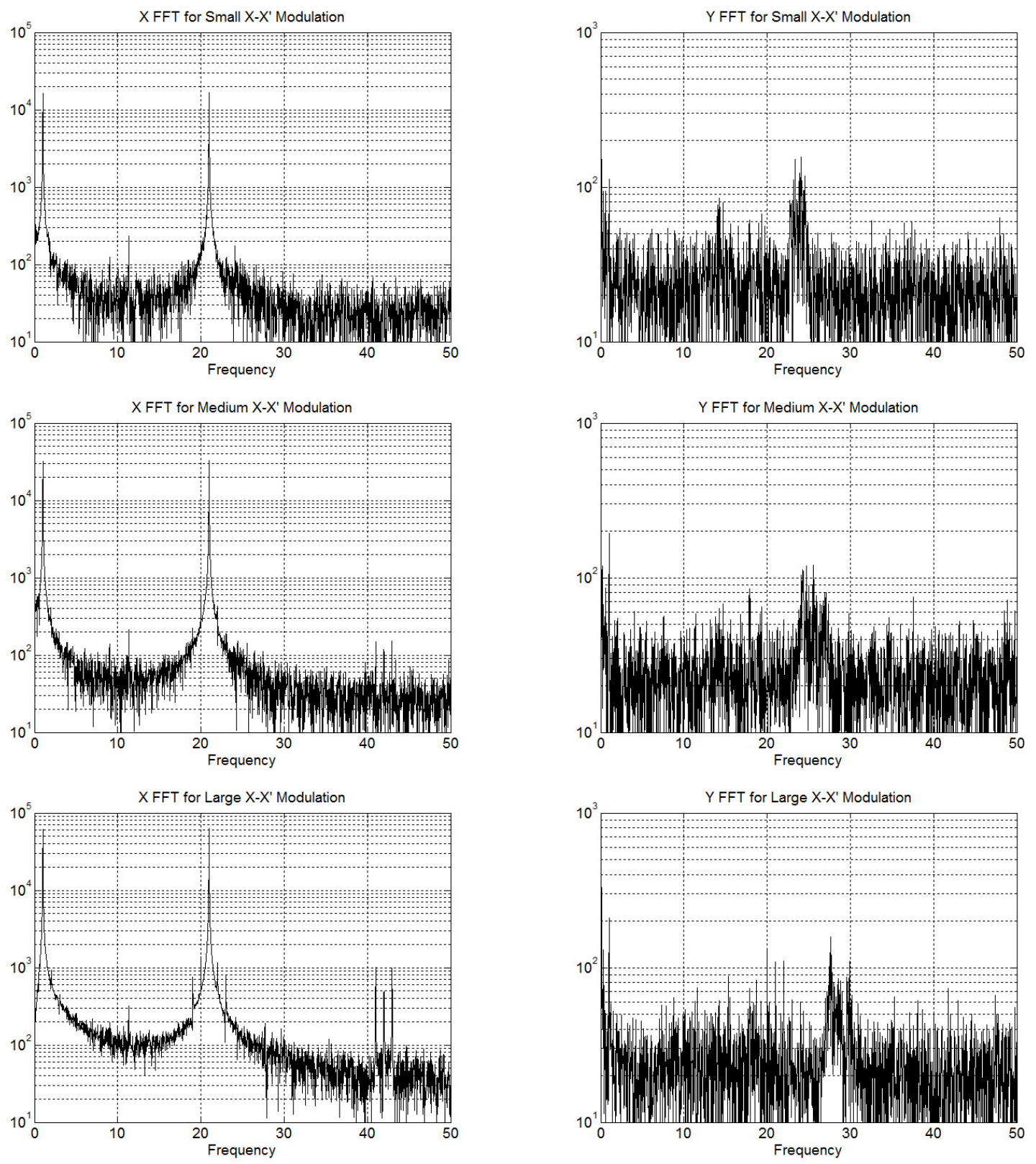

FIG. 66. Series of FFT plots of the 6T09 BPM for $x-x^{\prime}$ modulation. 

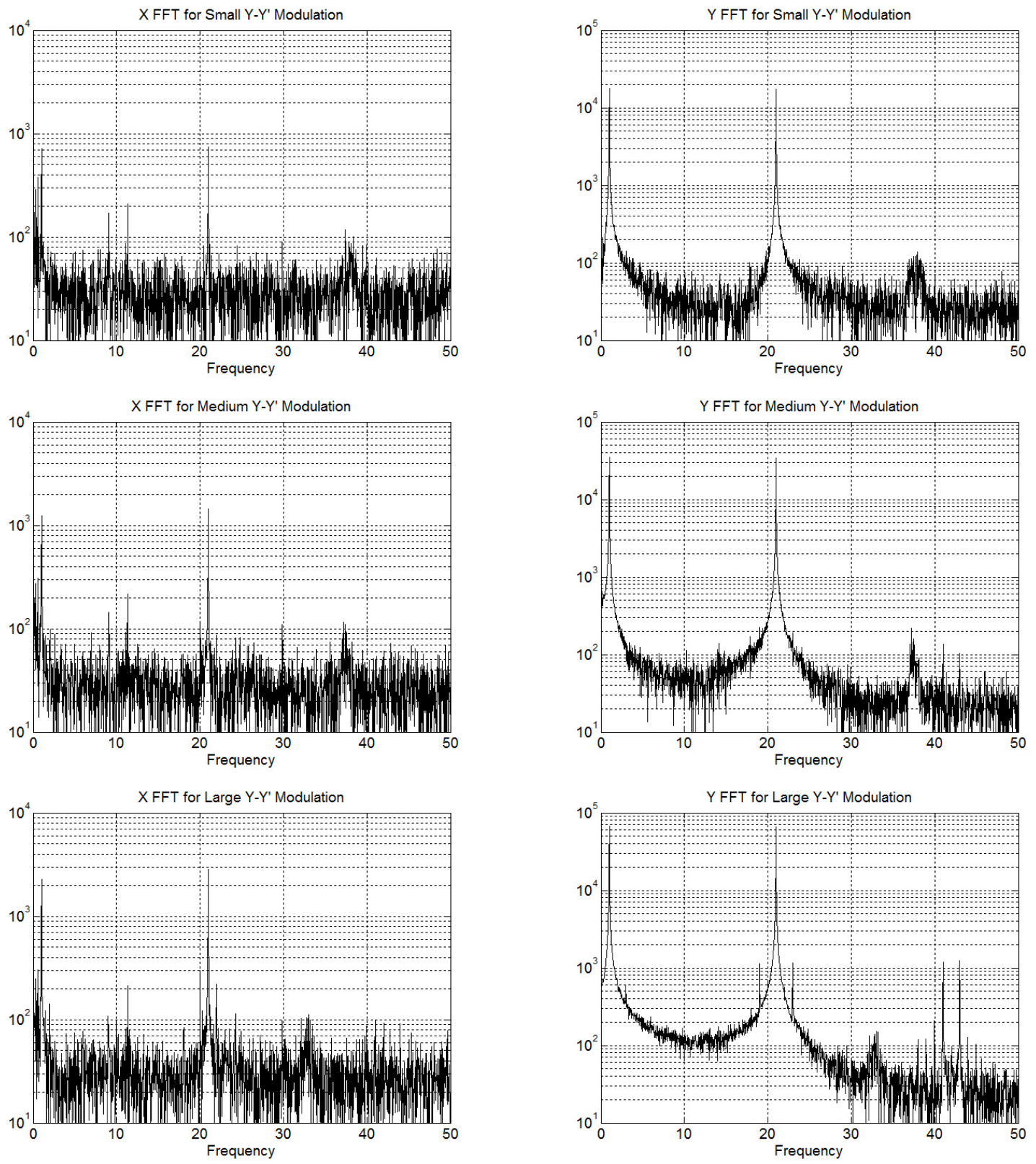

FIG. 67. Series of FFT plots of the 6T09 BPM for $y-y^{\prime}$ modulation. 


\section{CHAPTER 7}

\section{CONCLUSIONS}

\subsection{SUMMARY OF EXPERIMENT}

This experiment set out to study the feasibility of using simultaneous transverse beam modulation as a means of measuring the nonlinear fields of the magnets that make up a beam transport system. The intent was to develop a calibration standard with a known and controllable nonlinear sextupole and to then use the technique to characterize the nonlinear multipole content in dipoles as well as whole systems of magnets. Traditional methods of tuning the CEBAF accelerator have used linear techniques to tune the optics of the machine which are of course insensitive to nonlinear effects. The development of this technique is hoped to be able to extend our tuning capabilities to account for nonlinear effects when gauging the overall quality of the beam tune.

The derivation of the functional form of magnetic fields within dipoles, quadrupoles, sextupoles, and octupoles as well as the general multipole expansion provide a clear foundation for understanding the interaction of the beam with the Lorentz forces of the beam transport system. In particular we gain the ability to predict the expected harmonic content for different types of magnets based on the functional form of the fields as derived using Maxwell's equations.

Fundamental linear optics theory and the well-known matrix formalism for linear systems was presented to provide a basis for the development of an analytical model. This simple model was used to establish the expected frequencies for the harmonic content of sextupoles and octupoles when the beam is simultaneously modulated at two distinct frequencies.

Through the development of the Chebyshev formalism we have shown that the unique properties of this class of polynomials coupled with the orthogonality of Fourier cosine expansions allows us to perform modulation experiments with minimum error. With the application of precise modulation frequencies to the beam and the use of the NAFF algorithm to minimize peak detection errors we found good agreement between the theory, multiple models and the experimental results. 
The existing air-core kicker magnets in Arc 1 were well suited for this research. In general they were able to provide sufficient field at $1 \mathrm{~Hz}$ and $21 \mathrm{~Hz}$ as they were originally designed as part of a $30 \mathrm{~Hz}$ system. For the measurements in the Beam Switchyard Recombiner there were no magnets available. New magnets were designed, fabricated, tested and installed to perform this research. Using the Law of Biot-Savart a simple model of an air-core magnet was developed to come up with a working design. Bench measurements verified that the integrated dipole field strength was within $2 \%$ of the prediction. Thermal performance was also well within specification.

It was important to minimize the nonlinear fields of the kicker magnets so that their multipole content would not impact the results. The fabrication and assembly process successfully provided magnets with low harmonic content. In fact their performance in this regard was better than the magnets in Arc 1 despite them being physically longer. One thing that I had not considered during the design process was the effect of the higher inductance. Initial measurements showed a severe roll-off at low frequencies. A simple change to a gain resistor provided better matching and fixed the problem.

Both the existing magnets in Arc 1 and the new magnets were installed with a few degree roll about the beamline axis. This provides a small amount of cross-plane coupling. A better job could have been done to manage the error.

The nonlinear errors of the Beam Position Monitor System were corrected using a Poisson model. Good results were achieved across a $\pm 2 \mathrm{~cm}$ aperture in both planes with correction to better than 10 microns.

The data acquisition system required extensive work to integrate it into the measurement scheme. The use of I-Q sampling and the CORDIC algorithm worked well with the $499 \mathrm{MHz}$ beam micropulse structure. The end result provided exceptional performance with regard to the signal:noise ratio and overall repeatability of measurement results.

The results from the Magnet Measurement Facility and TOSCA simulations were used to predict the expected behaviour for dipole measurements. Both predicted an increase in multipole strength as a function of position which was confirmed with beam-based measurements.

The elegant software package was used to develop a model of the beamline. Simulations were then conducted for sextupole and dipole magnets. The model predicted 
that there should be a linear increase in harmonic content with sextupole excitation. The model also predicted an increase in harmonic content as a function of transverse position within a dipole. Both were confirmed with beam-based measurements.

The beam measurements for the sextupole calibration run and the elegant model both showed a linear dependence of amplitude with sextupole excitation for all harmonic sidebands. The difference in slope between beam measurements and the model can be attributed to a mismatch between the linear model and the real machine transport.

The beam measurements for the BE dipole magnets in Arc 1 verified the qualitative results from TOSCA, Magnet Measurement and elegant simulations. The amplitude of the sidebands increase as a function of transverse position in the dipole. The real machine aperture limited the extent to which this could be measured due to beam loss on the vacuum beam tube at large transverse position.

The measurements in the Beam Switchyard Recombiner indicate that within a $\pm 5 \mathrm{~mm}$ aperture the system is very linear. Larger amplitude excitations do indicate a departure from linearity. A detailed model of this system was not developed for comparison.

Overall the experiment was successful in measuring the nonlinear fields of sextupoles, dipoles and systems of magnets with good signal:noise.

\subsection{SUGGESTIONS FOR FUTURE WORK}

Looking forward, its likely that more measurements will be made as the $12 \mathrm{GeV}$ machine is commissioned. Some improvements in the methods used here and the upgrade of existing hardware will make measurements such as these more integrated in the accelerator. For example, the BPM electronics used here are now decommissioned. The new BPM systems that are being installed have onboard data acquisition hardware that rival what was used here and are distributed around the whole machine.

The nonlinear correction algorithm for the Beam Position Monitor System used here had not been implemented in the machine before this research. These algorithms are being built into the hardware which will make the model more precise. In particular the beam orbit in the Extraction regions are typically well outside the linear range of the system.

There are many complementary machine modeling techniques under development 
at Jefferson Lab that are all aimed at nailing down the linear model. Coupling this research with those activities will make commissioning the machine far more efficient and effective in the $12 \mathrm{GeV}$ era. 


\section{BIBLIOGRAPHY}

[1] C. Hovater et al., The CEBAF RF Separator System, in Proceedings of the 1996 Linear Accelerator Conference, Geneva, Switzerland, p. 77, CERN 96-07, 1996.

[2] K. Wille, The Physics of Particle Accelerators, Oxford University Press, Oxford, NY, first edition, 2000.

[3] H. Wiedemann, Particle Accelerator Physics 1, Springer, Berlin, Germany, second edition, 1999.

[4] P. L. Chebyshev, Théorie des mécanismes connus sous le nom de parallélogrammes, in Memoirs of Foreign Scholars presented at the Academy of St. Petersburg, volume 7, pp. 539-586, 1854.

[5] D. W. Kerst and R. Serber, Physical Review 60, 53 (1941).

[6] G. W. Hill, Acta Mathematica VIII, 1 (1886).

[7] Vector Fields 3D Magnetostatic Modeling Software., Cobham Technical Services, Aurora, IL.

[8] M. Borland, elegant: A Flexible SDDS-Compliant Code for Accelerator Simulation, Advanced Photon Source LS-287, (September 2000).

[9] M. A. Snyder, Chebyshev Methods in Numerical Approximation, p. 18, Prentice Hall, Inc. Series in Automatic Computation, Englewood Cliffs, NJ, 1966.

[10] M. A. Snyder, Chebyshev Methods in Numerical Approximation, p. 4, Prenticei Hall, Inc. Series in Automatic Computation, Englewood Cliffs, NJ, 1966.

[11] L. Debnath and P. Mikusiński, Introduction to Hilbert Spaces with Applications, Academic Press, Inc., San Diego, CA, first edition, 1990.

[12] R. Winter, Quantum Physics, pp. 227-230, Faculty Publishing, Inc., Davis, CA, second edition, 1986.

[13] M. A. Snyder, Chebyshev Methods in Numerical Approximation, p. 7, Prentice Hall, Inc. Series in Automatic Computation, Englewood Cliffs, NJ, 1966. 
[14] J. Laskar, C. Froeschlé, and A. Celletti, Physica D 56, 253 (1992).

[15] M. A. Snyder, Chebyshev Methods in Numerical Approximation, p. 31, Prenticei Hall, Inc. Series in Automatic Computation, Englewood Cliffs, NJ, 1966.

[16] T. W. Korner, Fourier Analysis, Cambridge University Press, Cambridge, GB, 1988.

[17] Data from the National Geophysical Data Center at www.ngdc.noaa.gov.

[18] A. S. Hofler et al., Performance of the Cebaf Arc Beam Position Monitors, in Proceedings of the 1993 Particle Accelerator Conference, Washington, DC, p. 93, IEEE Catalog No 93CH3279-7, 1993.

[19] W. A. Barry, A General Analysis of Thin Wire Pickups for High Frequency Beam Position Monitors, in Nuclear Instruments and Methods, pp. 407-416, 1991.

[20] T. Allison and T. Powers, CEBAF Distributed Data Acquisition System, in Proceedings of the 2005 Particle Accelerator Conference, Knoxville, TN, pp. 3541-3542, IEEE, 2005.

[21] J. E. Volder, The CORDIC Trigonometric Computing Technique, in IRE Transactions on Electronic Computers, volume EC-8, pp. 330-334, 1959.

[22] J. Musson and K. Cole, Application of Goubau Surface Wave Transmission Line for Improved Bench Testing of Diagnostic Beamline Elements, in Proceedings of the 2009 Particle Accelerator Conference, Vancouver, BC, pp. 4060-4062, IEEE, 2009 .

[23] A. Sommerfeld, Annalen Der Physik 303, 233 (1899).

[24] G. Goubau, Single-Conductor Surface-Wave Transmission Lines, in Proceedings of the IRE 39, pp. 619-624, IEEE, 1951.

[25] P. Piot, Evaluation and Correction of Nonlinear Effects in FNPL Beam Position Monitors, Technical Report Internal FNAL Pub, Fermilab, 2005.

[26] K. Halbach and R. F. Holsinger, Superfish-a computer program for evaluation of RF cavities with cylindrical symmetry, in Patricle Accelerator \%, pp. 213-222, 1976 . 
[27] G. H. Hoffstaetter and R. W. Helms, Phys. Rev. ST Accel. Beams 8, 062802 (2005).

[28] K. Baggett, L. Harwood, and T. Hiatt, 12 GeV Dipole Magnet Measurement Analysis using Curved Beam Trajectories, Technical Report JLAB-TN 07-050, JLAB, 2007.

[29] The measurement data for the BE dipole was taken from the CEBAF Magnet Measurement Database.

[30] The development of the TOSCA model of the BE dipole and the simulations were performed by Dr. Jay Benesch.

[31] J. Laskar, Frequency Map Analysis of an Hamiltonina System, in Workshop on Non-Linear Dynamics in Particle Accelerators, pp. 130-159, AIP Conference Proc., 1995. 


\section{APPENDIX A}

\section{ELEGANT SIMULATION FILES}

\section{A.1 ARC 1 LATTICE FILE}

The following text describes the beamline for simulating the ARC1 experiment as described in section 5.4. It includes all components on the beamline from the MAZ1S08H kicker to the IPM1A21 Beam Position Monitor. The settings in the model match the values that were in the control system during the beam tests.

MAZ1S08H: HKICK, L=0, KICK $=0$, TILT $=0$, STEERING $=0$

D116: DRIFT, L=5.08611

IPM1S08: MONITOR, $\mathrm{L}=0$

MQB1S08: KQUAD, $\mathrm{L}=0.15, \mathrm{~K} 1=1.521231, \mathrm{TILT}=0$

MBT1S08H: HKICK, L $=0, K I C K=0$, TILT $=0$

MBT1S08V: VKICK, L $=0, K I C K=0$, TILT $=0$

MAZ1S09V: VKICK, $L=0, K I C K=0$, TILT $=0$, STEERING $=0$

IPM1S09: MONITOR, L=0

MQB1S09: KQUAD, L=0.15, K1=-1.50963, TILT $=0$

MBT1S09V: VKICK, $\mathrm{L}=0, \mathrm{KICK}=0$, TILT $=0$

IPM1S10: MONITOR, L=0

MQB1S10: KQUAD, $L=0.15, K 1=7.410557 \mathrm{e}-01$, TILT $=0$

MBT1S10H: HKICK, L $=0, K I C K=0$, TILT $=0$

MBT1S10V: VKICK, $\mathrm{L}=0, \mathrm{KICK}=0, \mathrm{TILT}=0$

MAZ1E01H: HKICK, L $=0, K I C K=0, T I L T=0, S T E E R I N G=0$

MAZ1E01V: VKICK, L $=0$, KICK $=0$, TILT $=0$, STEERING $=0$

IPM1E01: MONITOR, L=0

MQB1E01: KQUAD, $L=0.15, K 1=-0.373350, T I L T=0$

MBT1E01H: HKICK, L $=0, K I C K=0, T I L T=0$

MBT1E01V: VKICK, $\mathrm{L}=0, \mathrm{KICK}=0$, TILT $=0$

D121: DRIFT, L=0.4803

MBW1E01: CSBEND, L=0.500137 \& 
, ANGLE $=-0.0405309085544383, \mathrm{~K} 1=0$ \&

, TILT $=0$ \&

, E1=0, HGAP $=0$, FINT $=0.5$ \&

, E2 $=-0.0405309085544383, E D G E \_O R D E R=1 \&$

, INTEGRATION_ORDER $=4$ \&

, N_KICKS $=20$

D122: DRIFT, L $=5.75472$

MBX1E02: CSBEND, L=1.00027 \&

, ANGLE $=0.0810616425759514, K 1=0$ \&

, TILT $=0$ \&

, $\mathrm{E} 1=0.0405309085544383, \mathrm{HGAP}=0, \mathrm{FINT}=0.5 \&$

, E2 $=0.0405309085544383, E D G E \_O R D E R=1 \&$

, INTEGRATION_ORDER $=4$ \&

, N_KICKS $=20$

MBW1E03: CSBEND, $\mathrm{L}=0.5001370000000001$ \&

, ANGLE $=-0.0405309085544383, \mathrm{~K} 1=0$ \&

, TILT $=0$ \&

, $\mathrm{E} 1=-0.0405309085544383, \mathrm{HGAP}=0, \mathrm{FINT}=0.5 \&$

, E2 $=0$, EDGE_ORDER=1 \&

, INTEGRATION_ORDER $=4$ \&

, N_KICKS $=20$

D123: DRIFT, L=0.525003

IPM1E02: MONITOR, L=0

MQB1E02: KQUAD, $\mathrm{L}=0.15, \mathrm{~K} 1=0.556967, \mathrm{TILT}=0$

MBT1E02H: HKICK, L $=0, K I C K=0$, TILT $=0$

MBT1E02V: VKICK, L=0, KICK $=0$, TILT $=0$

D124: DRIFT, L=15.6361

IPM1E03: MONITOR, L=0

MQB1E03: KQUAD, L=0.15, K1=-0.613577, TILT $=0$

MBT1E03H: HKICK, L $=0$, KICK $=0$, TILT $=0$

MBT1E03V: VKICK, $\mathrm{L}=0, \mathrm{KICK}=0$, TILT $=0$

D124A : DRIFT, L=0.36866

IHA1E03: MONITOR, L=0

D124B：DRIFT, L=15.2675 
IPM1A01: MONITOR, L=0

MQB1A01: KQUAD, $L=0.15, K 1=1.05041, \quad T I L T=0$

MBT1A01H: HKICK, L=0, KICK $=0, \operatorname{TILT}=0$

MBT1A01V: VKICK, L=0, KICK $=0, \operatorname{TILT}=0$

ITV1A01: MONITOR, L=0

D125: DRIFT, L=1.71272

MQB1A02: KQUAD, $\mathrm{L}=0.15, \mathrm{~K} 1=-0.288196, \mathrm{TILT}=0$

D126: DRIFT, L=2.68242

MKMATCH1S : MARK \&, FITPOINT $=1$

MBE1A01: CSBEND, $\mathrm{L}=1.00161 \&$

, ANGLE $=0.196349540849362, K 1=-0.00229840648881061 \&$

, TILT $=0$ \&

, $\mathrm{E} 1=0.09817477042468099, \mathrm{HGAP}=0.0127, \mathrm{FINT}=0.5 \&$

, E2=0.09817477042468099, EDGE_ORDER=1 \&

, INTEGRATION_ORDER $=4$ \&

, N_KICKS $=20$

D127: DRIFT, L=5.2152

IPM1A03: MONITOR, L=0

MQB1A03: KQUAD, L=0.15, K1=-1.16578, TILT $=0$

MBT1A03V: VKICK, L=0, KICK=0, TILT $=0$

D128: DRIFT, L=2.3809

D159: DRIFT, L=0.15

D129: DRIFT, L=0.21202

IPM1A04: MONITOR, L=0

MQB1A04: KQUAD, $\mathrm{L}=0.15, \mathrm{~K} 1=2.13112, \mathrm{TILT}=0$

MBT1A04H: HKICK, L=0, KICK=0, TILT=0

D130: DRIFT, L=0.70155

ITV1A04: MONITOR, L=0

D131: DRIFT, L=1.87544

IPM1A05: MONITOR, L=0

MQB1A05: KQUAD, L=0.15, $\mathrm{K} 1=-0.84544, \mathrm{TILT}=0$

MBT1A05V: VKICK, L=0, KICK $=0, \operatorname{TILT}=0$

D132: DRIFT, L=5.05061

MBE1A02: CSBEND, $\mathrm{L}=1.00161 \&$ 
, ANGLE $=0.196349540849362, \mathrm{~K} 1=-0.00229840648881061 \&$

, TILT $=0$ \&

, $\mathrm{E} 1=0.09817477042468099, \mathrm{HGAP}=0.0127, \mathrm{FINT}=0.5 \&$

, E2 $=0.09817477042468099, E D G E \_O R D E R=1 \&$

, INTEGRATION_ORDER=4 \&

, N_KICKS $=20$

IPM1A06: MONITOR, L=0

MQB1A06: KQUAD, $\mathrm{L}=0.15, \mathrm{~K} 1=0.79145, \mathrm{TILT}=0$

MBT1A06H: HKICK, L=0, KICK $=0$, TILT $=0$

D133: DRIFT, L $=4.54514$

MBE1A03: CSBEND, L=1.00161 \&

, ANGLE $=0.196349540849362, \mathrm{~K} 1=-0.00229840648881061 \&$

, TILT $=0$ \&

, $\mathrm{E} 1=0.09817477042468099, \mathrm{HGAP}=0.0127, \mathrm{FINT}=0.5 \&$

, E2 $=0.09817477042468099, E D G E \_O R D E R=1 \&$

, INTEGRATION_ORDER=4 \&

, N_KICKS $=20$

IPM1A07: MONITOR, L=0

MQB1A07: KQUAD, L=0.15, K1=-0.849233, TILT $=0$

MBT1A07V: VKICK, $\mathrm{L}=0, \mathrm{KICK}=0$, TILT $=0$

D134: DRIFT, L $=2.74293$

IPM1A08: MONITOR, L=0

MQB1A08: KQUAD, $L=0.15, K 1=1.56739$, TILT $=0$

MBT1A08H: HKICK, L=0, KICK $=0$, TILT $=0$

D135: DRIFT, L=2.93902

IPM1A09: MONITOR, L=0

MQB1A09: KQUAD, $L=0.15, K 1=-0.757331, T I L T=0$

MBT1A09V: VKICK, $\mathrm{L}=0, \mathrm{KICK}=0$, TILT $=0$

D136: DRIFT, L=5.0506

MBE1A04: CSBEND, L=1.00161 \&

, ANGLE $=0.196349540849362, K 1=-0.00229840648881061 \&$

, TILT $=0$ \&

, $\mathrm{E} 1=0.09817477042468099, \mathrm{HGAP}=0.0127, \mathrm{FINT}=0.5$ \&

, E2 $=0.09817477042468099, E D G E \_O R D E R=1 \&$ 
, INTEGRATION_ORDER $=4$ \&

, N_KICKS $=20$

D137: DRIFT, L $=2.38277$

IPM1A10: MONITOR, $\mathrm{L}=0$

D138: DRIFT, L $=2.38277$

IPM1A11: MONITOR, $\mathrm{L}=0$

MQB1A11: KQUAD, $\mathrm{L}=0.15, \mathrm{~K} 1=1.243258, \mathrm{TILT}=0$

MBT1A11H: HKICK, L $=0, K I C K=0, T I L T=0$

MBE1A05: CSBEND, L=1.00161 \&

, ANGLE $=0.196349540849362, \mathrm{~K} 1=-0.00229840648881061 \&$

, TILT $=0$ \&

, $\mathrm{E} 1=0.09817477042468099, \mathrm{HGAP}=0.0127, \mathrm{FINT}=0.5$ \&

, E2 $=0.09817477042468099, E D G E \_0 R D E R=1 \&$

, INTEGRATION_ORDER=4 \&

, N_KICKS $=20$

IPM1A13: MONITOR, L=0

MQB1A13: KQUAD, $\mathrm{L}=0.15, \mathrm{~K} 1=-0.897279, \mathrm{TILT}=0$

MBT1A13V: VKICK, $\mathrm{L}=0, \mathrm{KICK}=0$, TILT $=0$

MSB1A14: SEXT, L=0.15, K2 $=32.65, \mathrm{ORDER}=2, \mathrm{DX}=0.00$

IPM1A14: MONITOR, L=0

MQB1A14: KQUAD, $L=0.15, K 1=1.39555, T I L T=0$

MBT1A14H: HKICK, L $=0, K I C K=0$, TILT $=0$

D157: DRIFT, L $=1.87544$

D140: DRIFT, L $=0.51167$

MQB1A15: KQUAD, $\mathrm{L}=0.15, \mathrm{~K} 1=-0.854162, \mathrm{TILT}=0$

MBT1A15V: VKICK, $\mathrm{L}=0, \mathrm{KICK}=0$, TILT $=0$

MBE1A06: CSBEND, L=1.00161 \&

, ANGLE $=0.196349540849362, K 1=-0.00229840648881061 \&$

, TILT $=0$ \&

, $\mathrm{E} 1=0.09817477042468099, \mathrm{HGAP}=0.0127, \mathrm{FINT}=0.5$ \&

, E2 $=0.09817477042468099$, EDGE_ORDER=1 \&

, INTEGRATION_ORDER=4 \&

, N_KICKS $=20$

IPM1A16: MONITOR, L=0 
MQB1A16: KQUAD, $L=0.15, K 1=0.539361, \quad T I L T=0$

MBT1A16H: HKICK, L=0, KICK $=0$, TILT $=0$

MBE1A07: CSBEND, $L=1.00161 \&$

, ANGLE $=0.196349540849362, K 1=-0.00229840648881061 \&$

, TILT $=0$ \&

, $\mathrm{E} 1=0.09817477042468099, \mathrm{HGAP}=0.0127, \mathrm{FINT}=0.5 \&$

, E2=0.09817477042468099, EDGE_ORDER=1 \&

, INTEGRATION_ORDER $=4$ \&

, N_KICKS $=20$

D141: DRIFT, L=5.51485

MQB1A17: KQUAD, $\mathrm{L}=0.15, \mathrm{~K} 1=-1.00616, \mathrm{TILT}=0$

MBT1A17V: VKICK, L=0, KICK=0, TILT $=0$

IPM1A18: MONITOR, L=0

MQB1A18: KQUAD, $\mathrm{L}=0.15, \mathrm{~K} 1=1.29706, \mathrm{TILT}=0$

MBT1A18H: HKICK, L=0, KICK=0, TILT $=0$

D158: DRIFT, L=2.23747

IPM1A19: MONITOR, L=0

MQB1A19: KQUAD, L=0.15, K1=-0.5900030000000001, TILT $=0$

MBT1A19V: VKICK, L=0, KICK=0, TILT $=0$

MBE1A08: CSBEND, $L=1.00161 \&$

, ANGLE $=0.196349540849362, K 1=-0.00229840648881061 \&$

, TILT $=0 \&$

, $\mathrm{E} 1=0.09817477042468099, \mathrm{HGAP}=0.0127, \mathrm{FINT}=0.5 \&$

, E2=0.09817477042468099, EDGE_ORDER=1 \&

, INTEGRATION_ORDER $=4$ \&

, N_KICKS $=20$

D142: DRIFT, L=2.68242

IPM1A21: MONITOR, L=0

D1000: DRIFT, L=4.563783

D1001: DRIFT, L=0.522307

D1003: DRIFT, $\mathrm{L}=4.66066$

D1004: DRIFT, L=0.42545

D1005: DRIFT, $\mathrm{L}=14.8678$

D1006: DRIFT, L=0.2921 
D1007: DRIFT, L=0.47625

D117: DRIFT, L=4.46076

D118: DRIFT, L=0.62535

D119: DRIFT, $\mathrm{L}=14.5108$

D120: DRIFT, L=0.5

\section{A.2 ARC1 ELEGANT FILE}

The following text contains the instructions for performing the simulations of the experiment using the lattice of the previous section. The segments of the file are described in section 5.4.

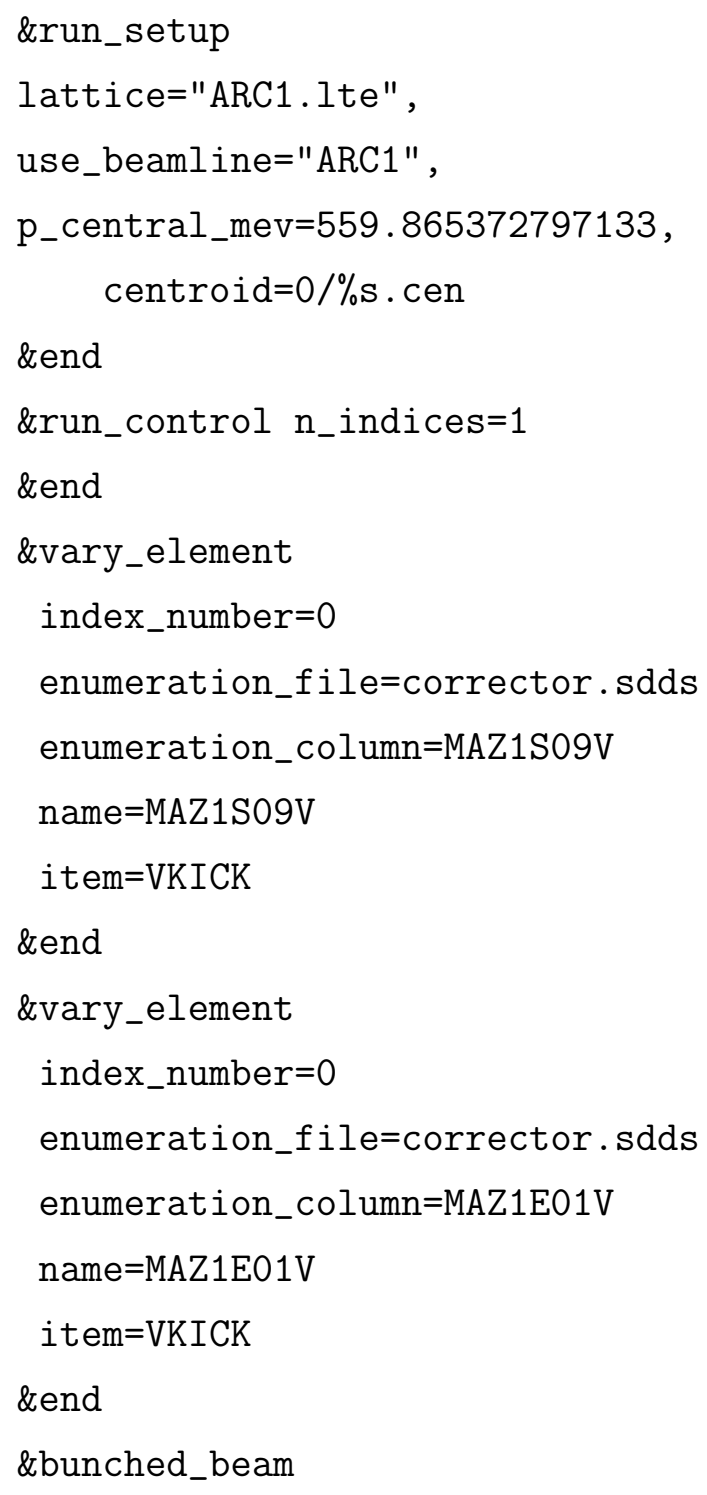




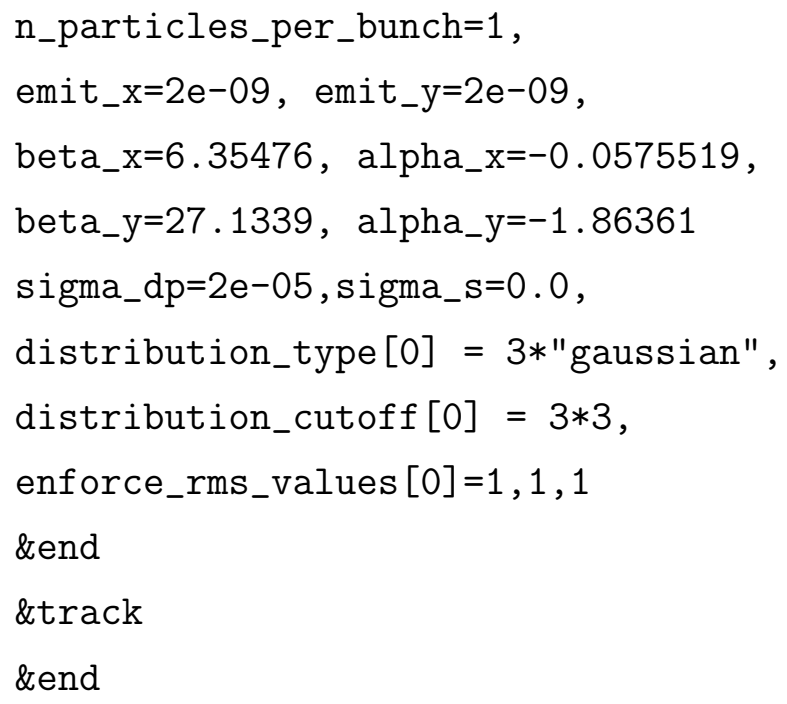




\section{APPENDIX B}

\section{MATLAB CODE FOR ANALYZING BPM WIRE DATA}

The following code was used to convert the raw data from the $32 \mathrm{BPM}$ wires to position data, rotate the position data to the lab frame and calculate the Fast Fourier transform and Power Spectral Density of the time domain data.

\%Convert BPM 4-channel wire data to properly rotated BPM positions $\%$ The data_in matrix contains the raw wire data $\%$ The BPM alpha values are read from the alpha matrix $\%$ The BPM names are read from the alphatext matrix $\%$ The sample rate is typically $500 \mathrm{~Hz}$.

$\%$

function $[\mathrm{x}, \mathrm{y}]=$ wires2fft_psd(data_in,alpha, alphatext, sample_rate) $\% \mathrm{k}=18.81$; $\quad \%$ Sensitivity for M15 BPM $\mathrm{k}=25.67$; $\quad \%$ Sensitivity for M20 BPM m=length (data_in); xrot=zeros (size(data_in)); \%reallocate memory yrot=zeros(size(data_in)); \%reallocate memory $\%$

\%Loop for converting wire data to position data $\%$ in rotated frame which is then rotated to the $\%$ laboratory frame $\%$

for $\mathrm{n}=(2: 4: 30)$

$\operatorname{xrot}(:, 0.25 * n+0.5)=k *($ data_in $(:, n)-a l p h a(0.25 * n+0.5,1)$ *data_in $(:, \mathrm{n}+1)) . /($ data_in $(:, \mathrm{n})+$ alpha $(0.25 * \mathrm{n}+0.5,1) *$ data_in $(:, \mathrm{n}+1))$; $\operatorname{yrot}(:, 0.25 * n+0.5)=k *($ data_in $(:, n+2)-a l p h a(0.25 * n+0.5,2)$ *data_in $(:, \mathrm{n}+3)) . /($ data_in $(:, \mathrm{n}+2)+$ alpha $(0.25 * \mathrm{n}+0.5,2) *$ data_in $(:, \mathrm{n}+3))$; $\mathrm{x}=\cos (\mathrm{pi} / 4) * \mathrm{xrot}-\mathrm{sin}(\mathrm{pi} / 4) *$ yrot; $\%$ rotate to lab frame $\mathrm{y}=\cos (\mathrm{pi} / 4) * \mathrm{yrot}+\sin (\mathrm{pi} / 4) * \operatorname{xrot} ; \%$ rotate to lab frame $\%$ 


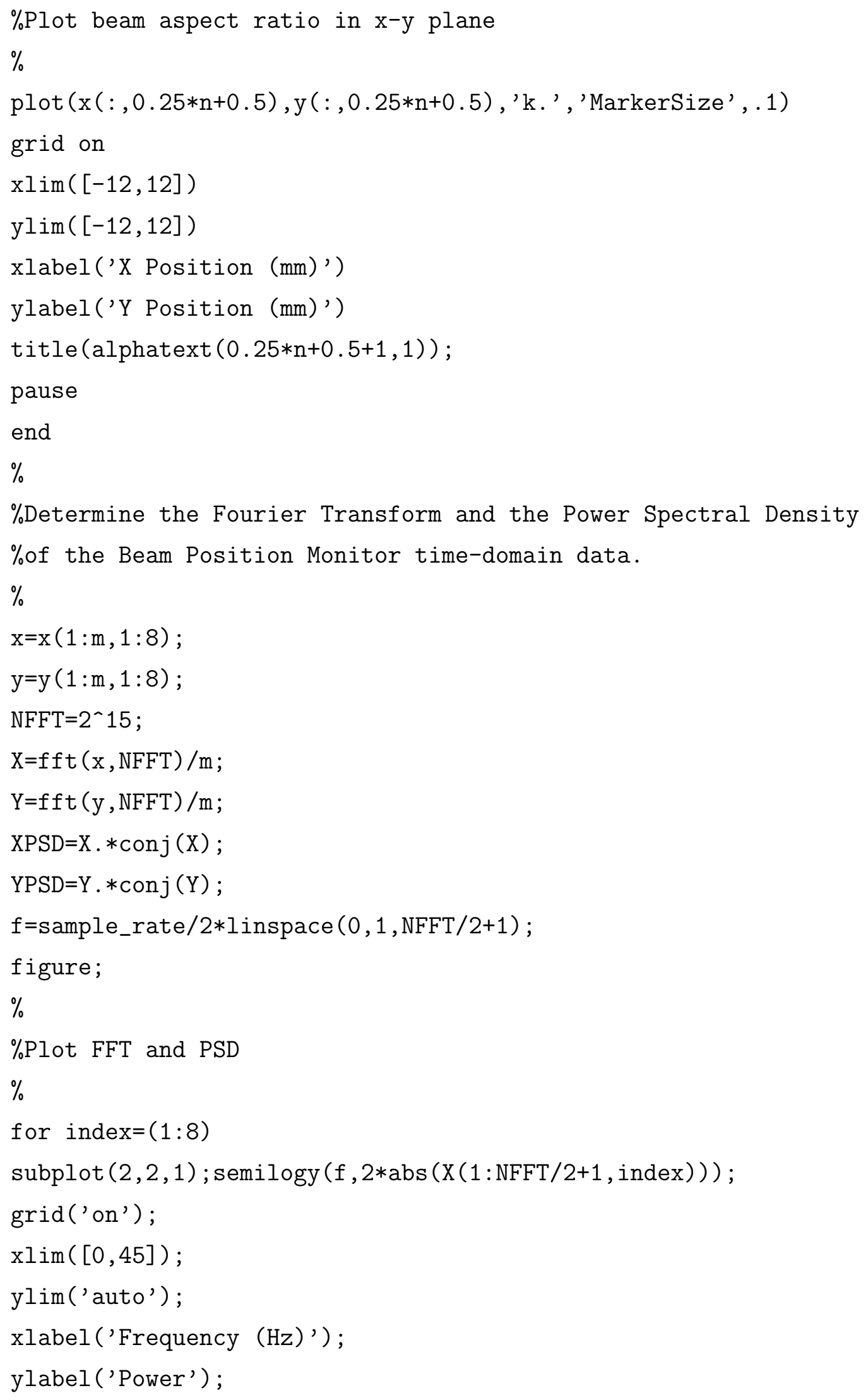




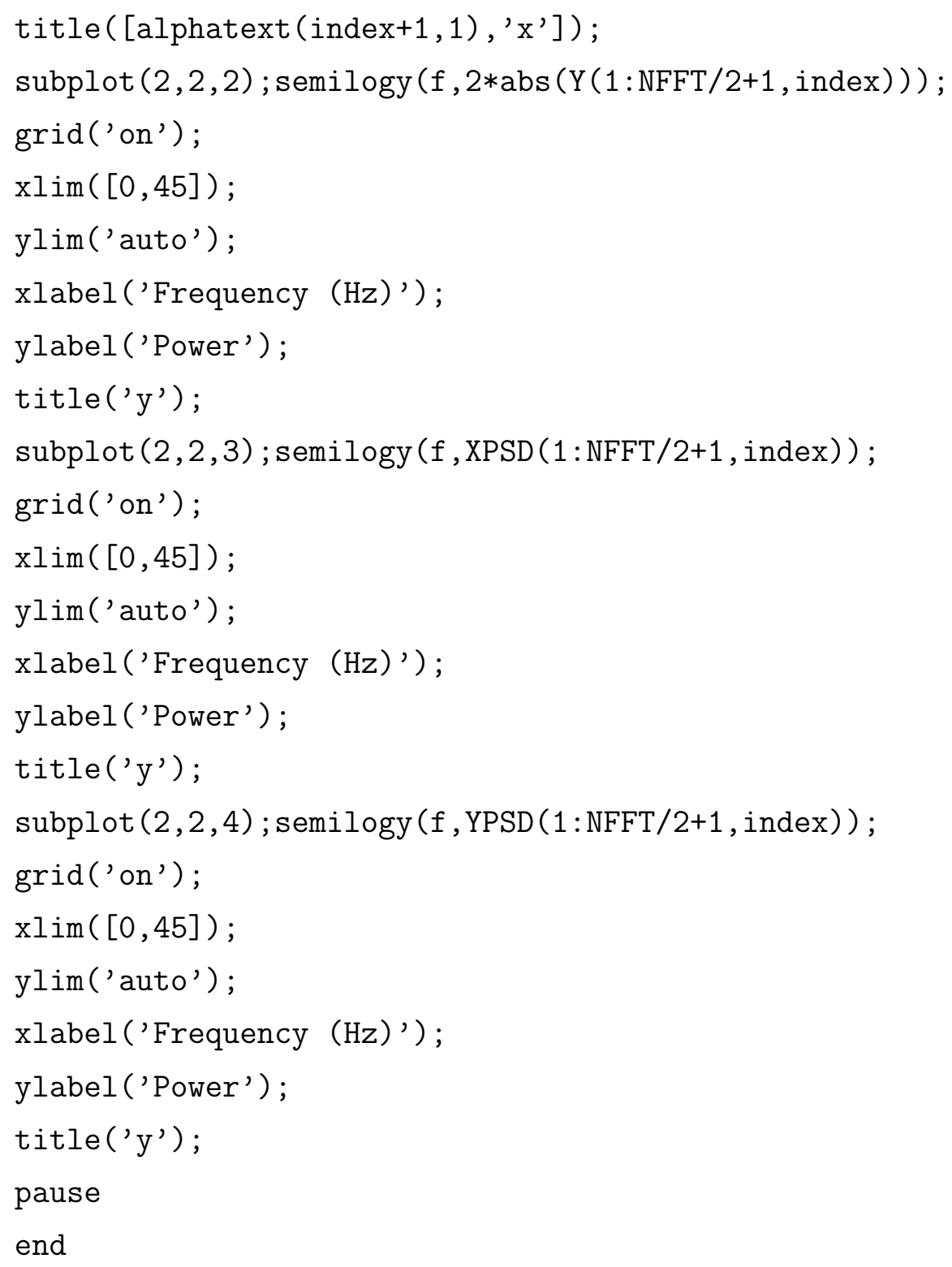




\section{APPENDIX C}

\section{POISSON CODE FOR SIMULATING BPM}

The following text shows the Poisson Automesh file which defines the physical dimensions of the BPM can as well as the position of one of the antennae.

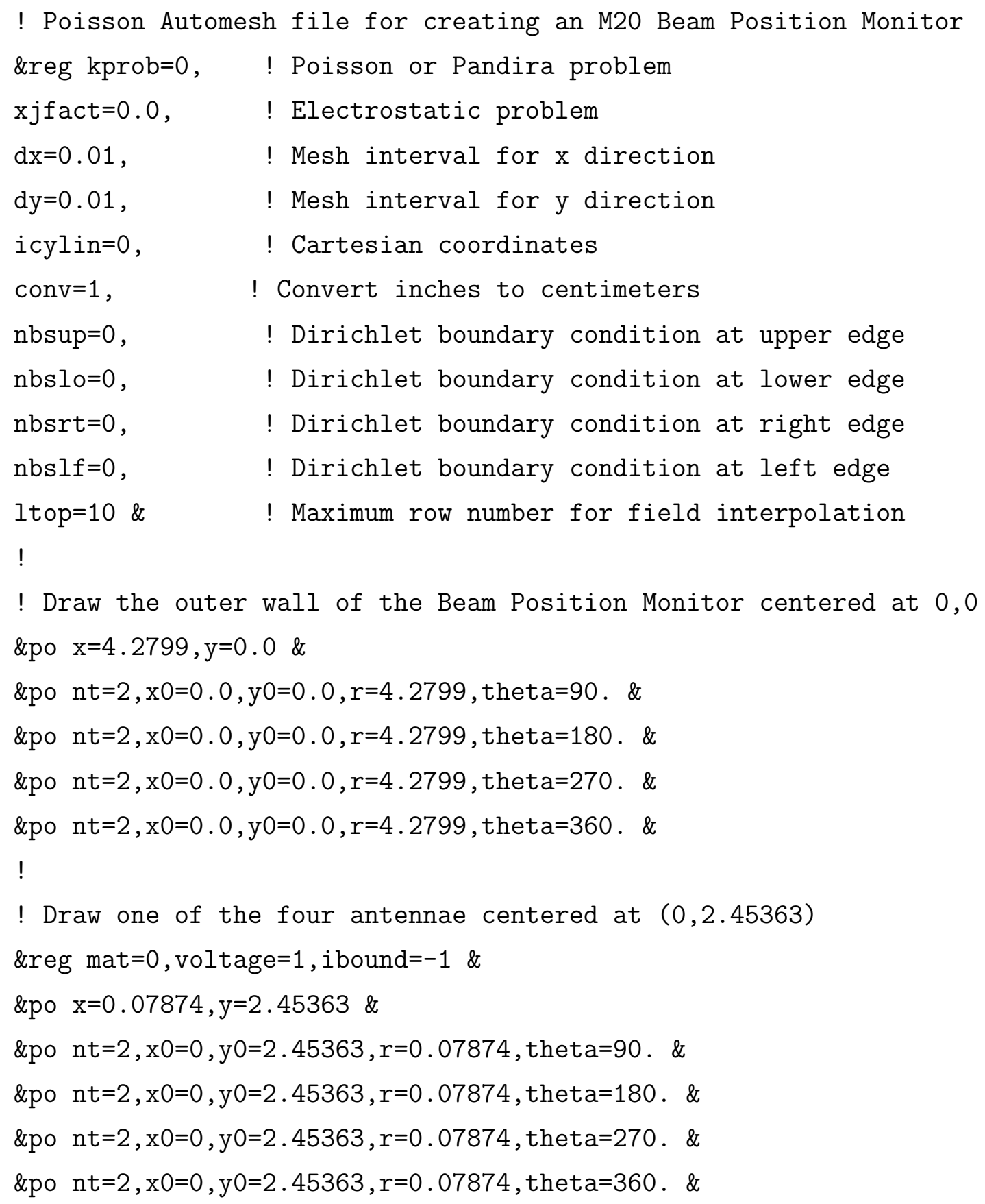




\section{VITA}

Michael Spata

Department of Physics

Old Dominion University

Norfolk, VA 23529

Michael Spata was born on October 2, 1961 in Staten Island New York. He was raised by his parents Joseph and Christine Spata along with an older brother Joe and younger sister Lisa. While an undergraduate at Stony Brook University from 19851990 he worked as a laboratory technician at the Nuclear Structure Laboratory's $10 \mathrm{MeV}$ Van de Graff ion accelerator. In the summer of 1989 he was given an opportunity to work as a summer intern with the Superconducting Radio Frequency Department (SRF) at the Continuous Electron Beam Accelerator Facility (CEBAF). During that time he was a member of the first cryomodule team which was responsible for building the prototype accelerating structure for the CEBAF linear accelerators. After graduating from Stony Brook with Bachelors of Science degrees in Physics and Astronomy he returned to CEBAF to work in the SRF Department from 19901993 testing ceramic windows for the niobium cavities, characterizing cavity pair performance in the Vertical Test Area, and ultimately joining the commissioning team as full cryomodule assemblies were delivered to the CEBAF site. In 1993 Mike joined the Operations Department as an Accelerator Operator and rose through the ranks to ultimately become the Operations Group Leader in 2001. In 2007 he joined the Center for the Advanced Studies of Accelerators at Jefferson Lab as a Staff Scientist and has since been working on the CEBAF $12 \mathrm{GeV}$ upgrade and providing support for day-to-day machine operations. Mike entered the graduate program at Old Dominion University in 2006 and received a Masters of Science degree in Physics in 2008. Mike met his future wife Sarah Tatum at CEBAF in 1993 and they were married in 1995. They have two daughters, Samantha and Emma, who were born in 1998 and 2001.

Typeset using $\mathrm{ATEX}_{\mathrm{E}}$ 